ANL-92/2

Volume II

\title{
COMMIX-PPC: A Three-Dimensional Transient Multicomponent Computer Program for Analyzing Performance of Power Plant Jondensers
}

Volume II: User's Guide and Manual

Manuscript Completed: August 1991

Date Published: February 1993

Prepared by

T. H. Chien, H. M. Domanus, and W. T. Sha

Materials and Components Technology Division

ARGONNE NATIONAL LABORATORY

9700 South Cass Avenue

Argonne, IL 60439

Prepared for

Taiwan Power Compingy

Taipel, Taiwan

Republic of China

DISTRIBUTION OF THIS DOCUMENT IS UNLIMTIED

DISCLAIMER

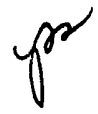

This report was prepared as an account of work sponsored by an agency of the United States Government. Neither the United States Government nor any agency thereof, nor any of their employees, makes any warranty, express or implied, or assumes any legal liability or responsibility for the accuracy, completeness, or usefulness of any information, apparatus, product, or process disclosed, or represents that its use would not infringe privately owned rights. Reference herein to any specific conmercial product, process, or service by trade name, trademark, manufacturer, or otherwise does not necessarily constitute or imply its endorsement, recommendation, or favoring by the United States Government or any agency thereof. The views and opinions of authors expressed herein do not necessarily state or reflect these of the United States Government or any agency thereof. 
As approved by the U.S. Nuclear Regulatory Commission and the U.S. Department of Energy, the COMMIX-PPC computer code was developed by modifying the in-house version of the COMMIX-2A computer code. The development of COMMIX-PPC is a team effort and the partictpants are listed below according to their activities.

Documentaion:

COMMLX-2A Version:

Code Programming and Development:

Overall Project Direction and Management:
T. H. Chien and W. T. Sha

T. H. Chien, H. M. Domanus, and W. T. Sha

T. H. Chien, H. M. Domanus, and W. T. Sha W. T. Sha 


\title{
COMMIX-PPC: A Three-Dimensional Transient Multicomponent Computer Program for Analyzing the Performance of Power Plant Condensers
}

\author{
Volume II: User's Guide and Manual
}

Abstract

The COMMIX-PPC computer program is an extended and improved version of earlier COMMIX codes and is specifically designed for evaluating the thermal performance of power plant condensers. The COMMIX codes are general-purpose computer programs for the analysis of fluid flow and heat transfer in complex industrial systems. In COMMIX-PPC, two major features have been added to previously published COMMIX codes. One feature is the incorporation of one-dimensional conservation of mass, momentum, and energy equations on the tube side, and the proper accounting for the thermal interaction between shell and tube side through the porous medium approach. The other added feature is the extension of the three-dimensional conservation equations for shell-side flow to treat the flow of a multicomponent medium.

COMMIX-PPC is designed to perform steady-state and transient three-dimensional analysis of fluid flow with heat transfer in a power plant condenser. However, the code is designed in a generalized fashion so that, with some modification, it can be used to analyze processes in any heat exchanger or other single-phase engineering applications.

The following unique features are retained from other COMMIX codes:

- Porous-Medium Formulation. COMMIX-PPC uses a new porous-medium formulation with the parameters of volume porosity, directional surface porosity, distributed resistance, and distributed heat source or sink. With this formulation, the COMMIX code can model an anisotropic flow domain with stationary structures, and it can be used to treat irregular geometries. The porous-medium formulation with the additional parameter of directional surface porosity represents a unified approach to thermal-hydraulic analysis. Because of this feature, it is now possible to perform a multidimensional thermal-hydraulic simulation of either a single engineering component, such as a rod bundle, reactor plenum, or piping system, or of a multicomponent system that is a combination of two or more engineering components.

- New Finite-Volume Formulation for equations of Conservation of Mass, Momentum, and Energy Equations. The momentum formulation employs the concept of a volume-averaged velocity as used in COMMIX-1C. It makes the numerical calculation more robust than in previous COMMIX versions. It also makes the location of pressure change coincide with that of density change for one-dimensional flows. In addition, the new discretized momentum equations also satisfy the one-dimensional Bernoulli equation.

- Three Matrix Solvers. In COMMIX-PPC, three matrix solvers, the successive overrelaxation method, the direct matrix inversion method, and the 
preconditioned conjugate gradient method for a symmetric matrix, are available to solve the pressure equation and scalar transport equations.

Depending on the size of the computational domain, the user can choose the solver that is best suited for a given problem. These three matrix solvers greatly increase the flexibility and efficiency of numerical computation for COMMIX-1C compared to previous codes.

- Geometrical Package. A special geometrical package has been developed and implemented to permit modeling of any complex geometry in the most storage-efficient way.

Volume I (Equations and Numerics) of this report describes in detail the basic equations, formulation. solution procedures, and models for auxiliary phenomena. Volume II (User's Guide and Manual) contains the input instruction, flow charts, sample problems, and descriptions of available options and boundary conditions.

COMMIX-PPC is a product of the continuing evolution of the family of COMMIX codes. The technical contents of the latest version of COMMIX-1C. when appropriate and relevant. will be duplicated here. 


\section{CONTENTS}

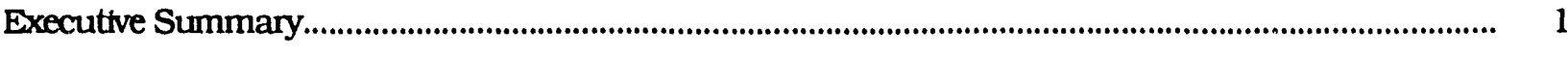

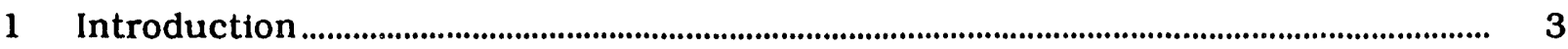

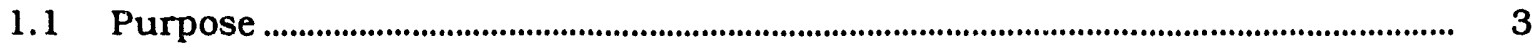

1.2 Organization of the Report............................................................................................. 4

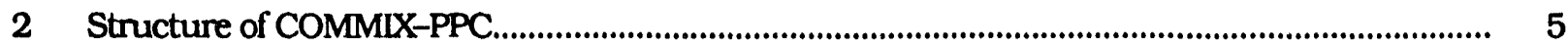

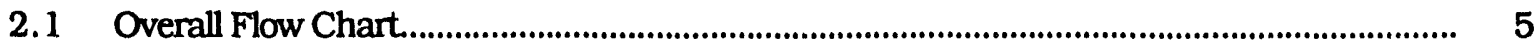

2.2 Solution Sequence........................................................................................................................ 5

2.3 Shell- and Tube-side Heat Transfer Coupling Loop................................................. 9

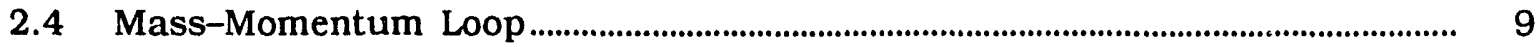

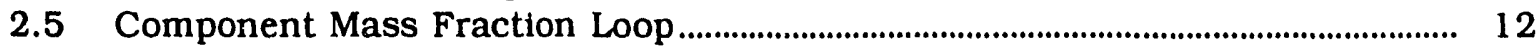

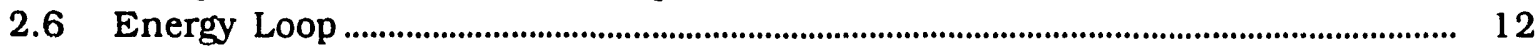

2.7 Tube-side Flow and Temperature Loop.......................................................... 13

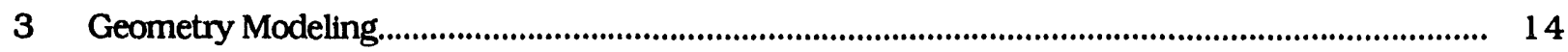

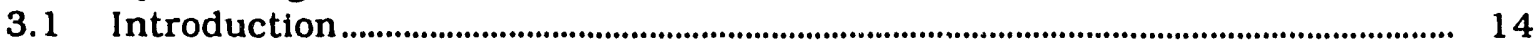

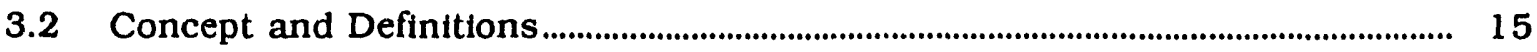

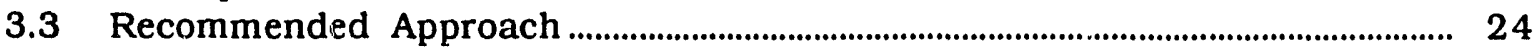

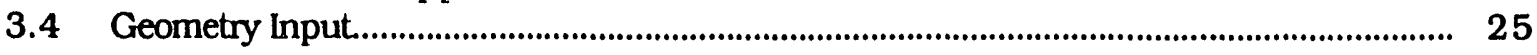

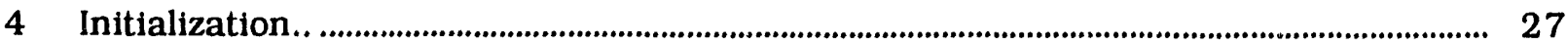

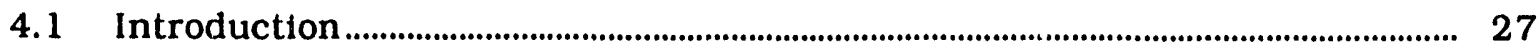

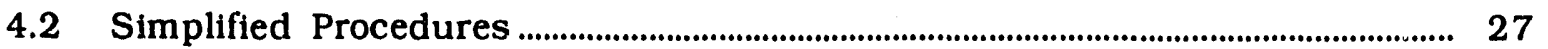

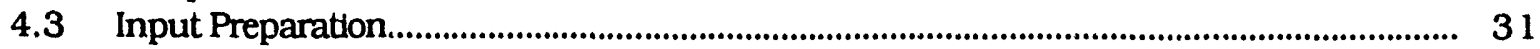

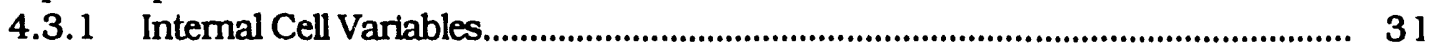

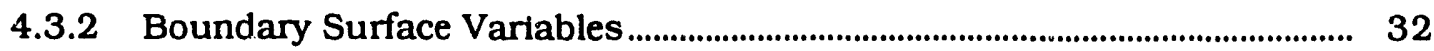

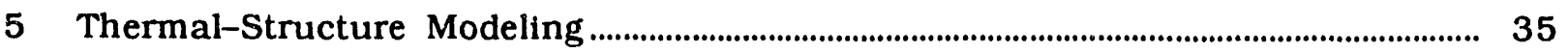

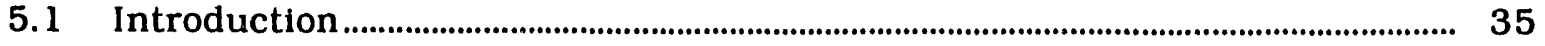

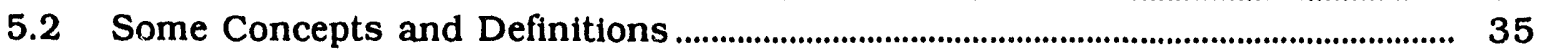

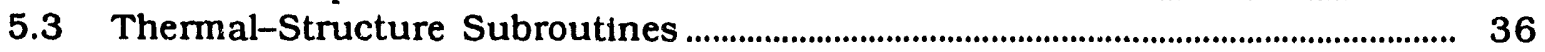

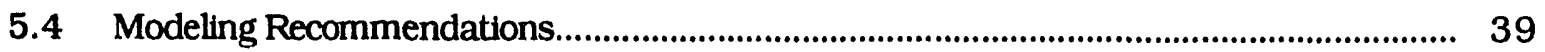

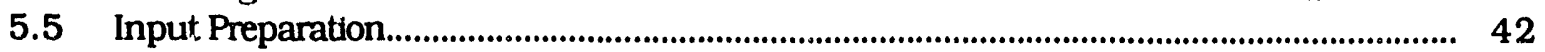

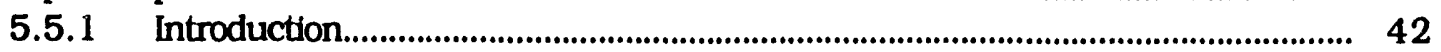

5.5.2 NAMELIST-In

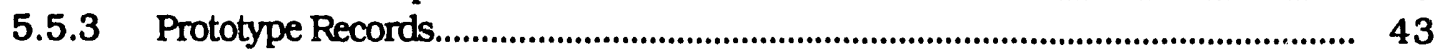

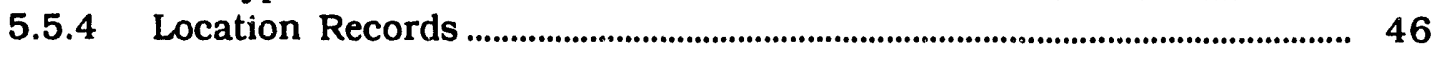

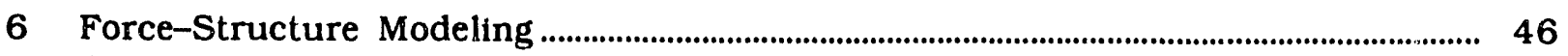

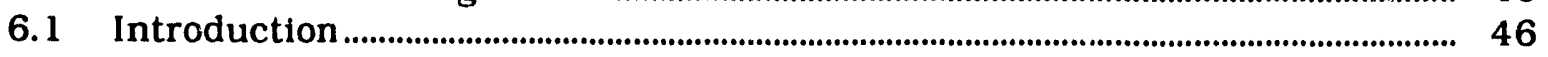

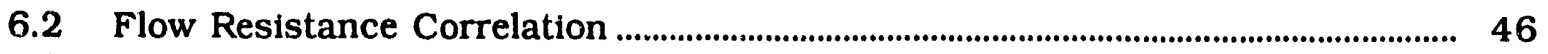

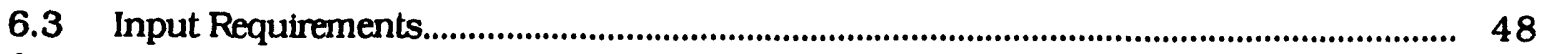

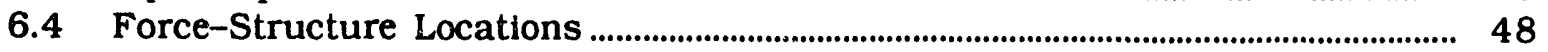

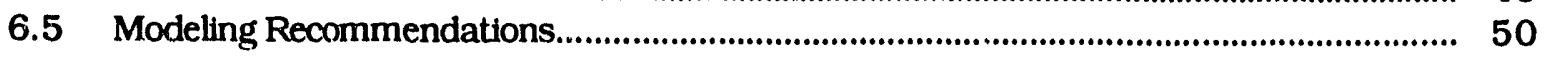

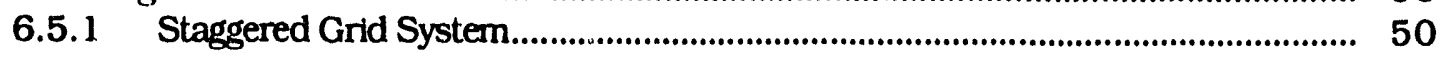

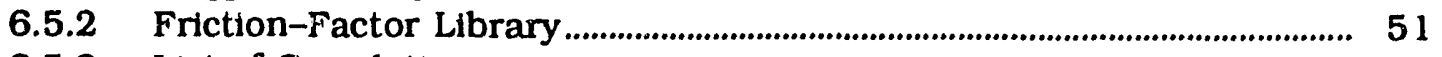

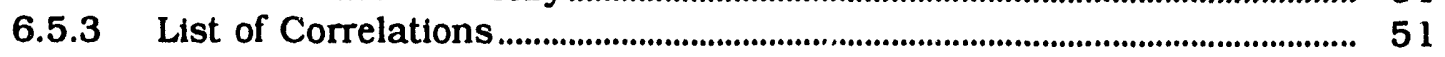

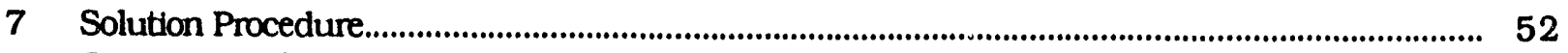

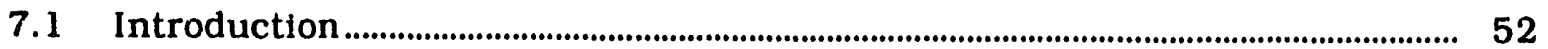


7.2 Solution Methods ........................................................................................................................ 52

7.3 Convergence Parameters .......................................................................................................... 53

7.4 Relaxation Parameters ................................................................................................ 54

7.4.1 Implicit Underrelaxation................................................................................. 54

7.4.2 Successive Overrelaxation .............................................................................. 55

8 Auxdliary Input............................................................................................................... 56

8.1 Heat Transfer Correlation ................................................................................... 56

8.2 Fluid and Material Properties ……............................................................................. 57

8.3 Turbulence Modeling .................................................................................................................. 59

8.3.1 Signal Parameter............................................................................................ 59

8.3.2 Constant-Diffustvity Model.......................................................................... 59

8.3.3 k-ع Two-Equation Turbulence Model....................................................... 59

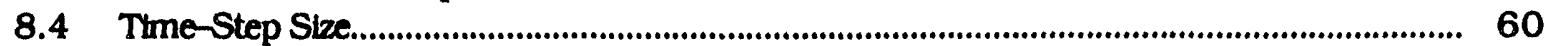

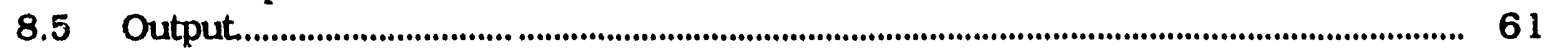

9 Steady-State Calculation ………....................................................................................................... 61

9.1 Introduction ........................................................................................................................ 61

9.2 Input Preparation.............................................................................................................. 62

9.3 Steady-State Convergence Criteria.................................................................................... 63

10 Transient Calculations....................................................................................................................... 64

10.1 Introduction............................................................................................................................ 64

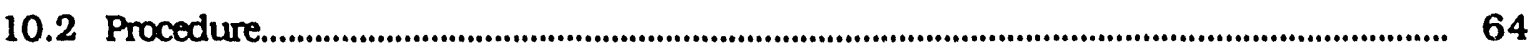

10.3 Transient Functions ........................................................................................................... 65

11 Operating COMMiX-PPC .................................................................................................................. 66

11.1 Creation of a Load Module ............................................................................................... 66

11.2 Input/Output...................................................................................................................................... 66

11.2 .1 Input Data Fle 5............................................................................................ 66

11.2.2 Printed Output File 6............................................................................... 66

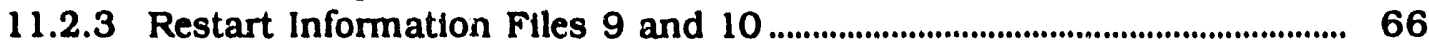

11.2 .4 Plot Tape Flle 76............................................................................................ 67

11.3 Error Detection and Diagnostics ....................................................................................... 67

12 Global Mass and Enthalpy Balances ...................................................................................... 67

12.1 Global Mass Balance ................................................................................................... 67

12.2 Global Enthalpy Balance ................................................................................................................ 68

13 Concluding Remarks........................................................................................................................ 69

Acknowledgments ............................................................................................................................................ 70

Appendix A: COMMIX-PPC Input Description …………………............................................................... 71

Appendix B: List of COMMIX-PPC Subroutines ..................................................................................... 119

Appendix C: Flow-Resistance Correlations ....................................................................................... 127

Appendix D: Sample Problem: Steady-State Performance of a Power Plant Condenser in the Presence of Noncondensible Air ............................................................................. 141

Appendix E: Sensitivity Study: Effect of Condenser Inlet Air Mass Fraction and Exit Mass Flow Rate of Air Extraction System on Steam Condensation Rate. 


\section{FIGURES}

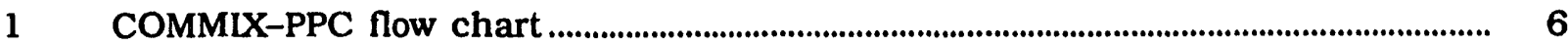

$2 \quad$ Flow chart of solution sequence in subroutine TIMSTP ..................................................... 8

3 Flow diagram of shell- and tube-side heat transfer coupling loop......................... 10

$4 \quad$ Flow diagram for mass-momentum loop ......................................................................... 11

$5 \quad$ Flow diagram for component mass fraction loop.......................................................... 13

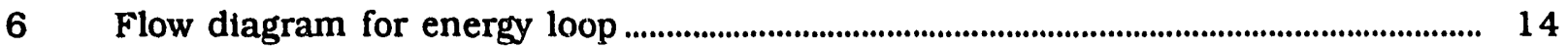

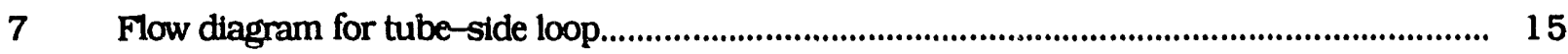

$8 \quad$ Partitioning in Cartesian and cylindrical coordinate systems .................................... 16

$9 \quad$ Model geometry showing boundary surfaces and partitioning of

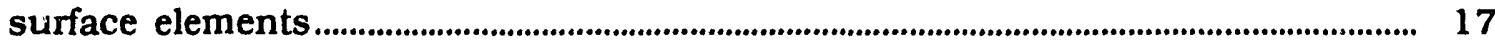

10 Model geometry showing irregular surface and partitioning of

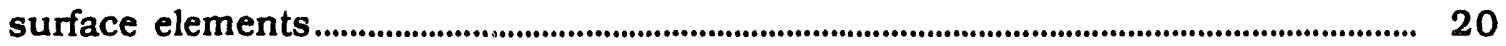

11 Two noncoinctdent surfaces with the same surface number.................................. 21

12 Two coineident surfaces with different surface numbers ............................................ 21

13 Volume porosity due to irregular geometry and internal

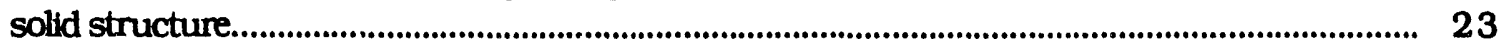

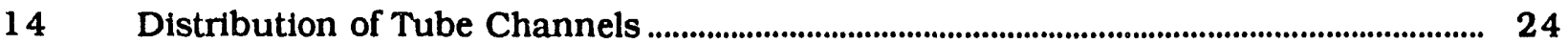

15 Illustrations showing partitioning of sample geometries............................................... 26

$16 \quad$ Illustrations showing thermal structure alignment...................................................... 36

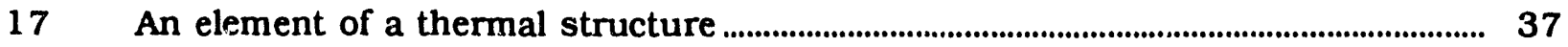

18 Element of a thermal structure, showing outer and inner surfaces ............................ 37

19 Four quarter-cylindrical structures, each interacting with one fluid cell ............................................................................................................................ 38

$20 \quad$ Multiple structures interacting with a single fluid cell.......................................... 38

21 Typical structure element showing material regions and gaps ................................... 38

22 Modeling multiple structures or fraction of a structure as one thermal structure .............................................................................................................................. 40

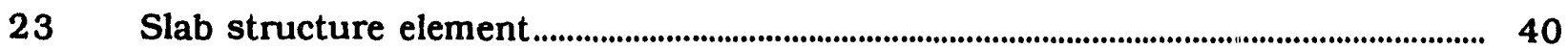

24 Uniformly distributed rod bundles in a nonuniform grid....................................... 42 


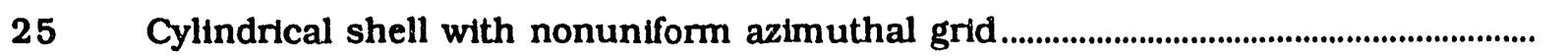

$26 \quad$ Numbering system of thermal-structure material regions................................... 45

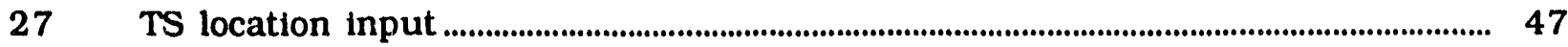

$28 \quad \mathrm{x}$-Momentum control volume in a staggered-grid system ............................................ 51

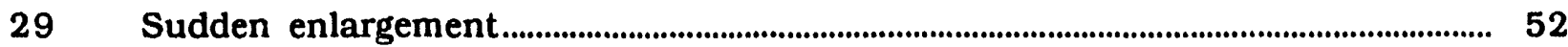

C.1 Nomenclature for staggered tube arrangement ......................................................................... 128

C.2 Nomenclature for in-line tube arrangement ........................................................................... 128

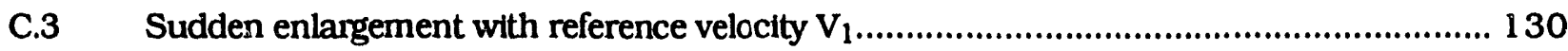

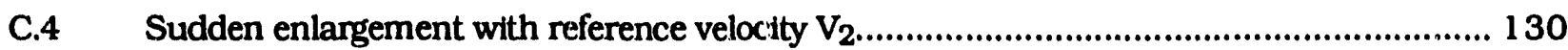

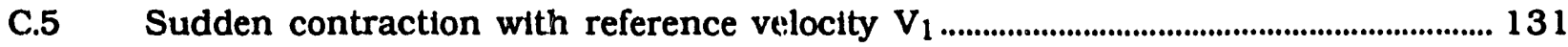

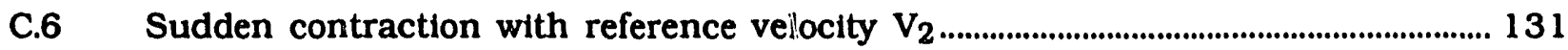

C.7 Venturi in a momentum control volume, where the loss coefficient

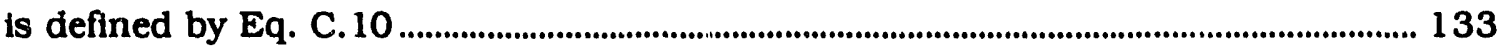

C.8 Venturt in a momentum control volume, where the loss coefficient

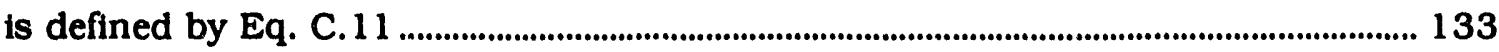

C.9 Nozzle in a momentum control volume ......................................................................... 134

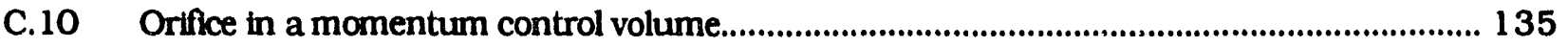

C.11 Discharge coefficient for VDI nozzle............................................................................ 136

C.12 Discharge coefficient for VDI orifice .......................................................................... 136

C.13 Submerged object in a momentum control volume...................................................... 137

C.14 Drag coefficient for several simple shapes ........................................................................... 137

C.15 Straight duct in a momentunı control volume............................................................ 139

C.16 Friction coefficients for fully developed laminar flow in rectangular tubes............. 139

D.1 Grid structure of the sample problem

D.2 Grid layout at the $I=4$ plane

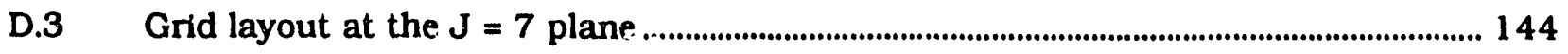

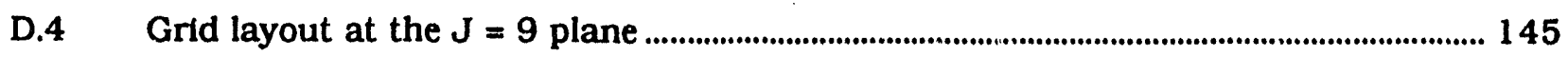

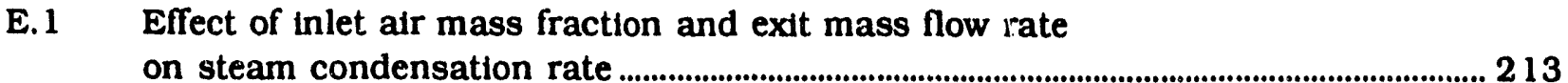




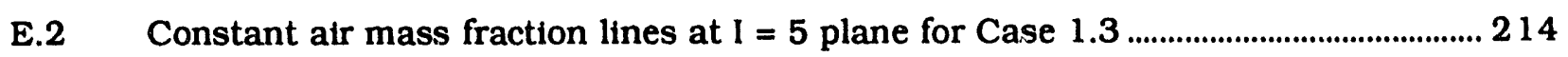

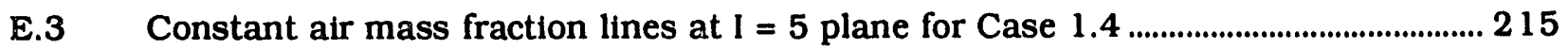

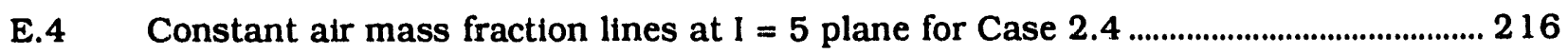

\section{TABLES}

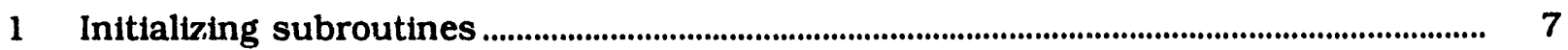

2 Components of unit normal vectors of geometry in Fig. 9a ......................................... 18

3 Initialization variables for uniform shell-side conditions .............................................. 28

4 Initialization variables for nonuniform shell-side conditions ........................................ 29

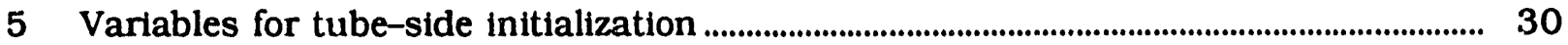

6 Options for shell-side velocity boundary conditions .................................................... 33

7 Options for shell-side temperature/heat flux boundary conditions................................ 34

8 Options for shell-side pressure boundary conditions ................................................... 34

9 Shell-side variables that can be specified through SEIR............................................ 34

10 FORTRAN variables for TS prototype records......................................................... 45

11 Input variables related to shell-side force-structure modeling ....................................... 49

12 Input variables related to tube-side force-structure modeling ......................................... 50

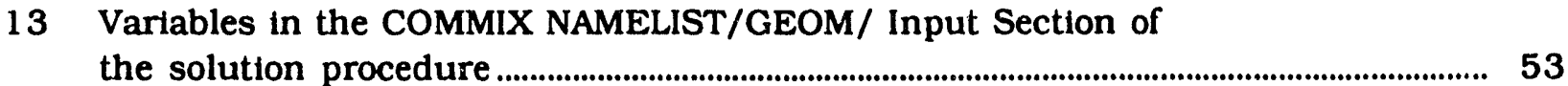

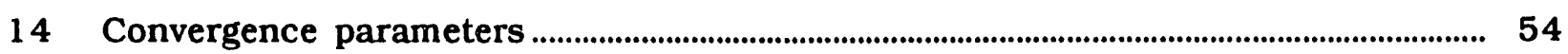

15 Underrelaxation parameters and default values employed in COMMIX-PPC .............. 55

16 Relaxation parameters and default values when SOR method is

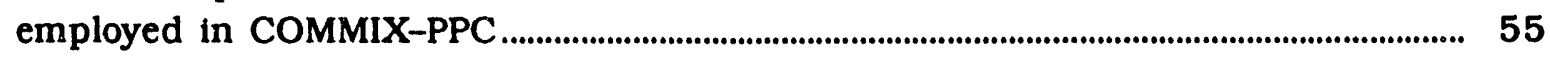

17 Coefficients employed in $k-\varepsilon$ two-equation turbulence model...........................................6 60

18 Possible additional input for $k-\varepsilon$ two-equation turbulence model .................................... 60

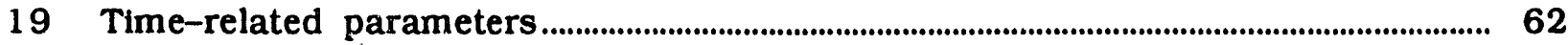

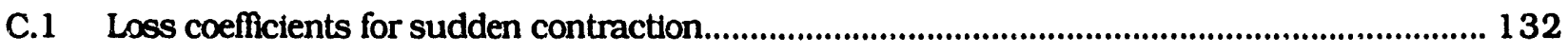

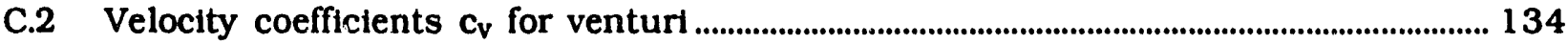

C.3 Head loss coefficients $\mathrm{K}$ for various fittings ................................................................... 140

E.1 Effect of condenser inlet air mass fraction and exit mass flow rate of an air extraction system on steam condensation rate 
The COMMIX (Component Mixing) code is a general-purpose computer program for the analysis of fluid flow in real-world engineering systems. Because the needs of users have changed since its inception in 1976, the code has undergone several stages of developnient, and several versions/extensions are now avallable. The present COMMIX-PPC is yet another extension, designed specifically for the purpose of analyzing the performance of power plant condensers. To meet this objective, two major additions have been incorporated into the code:

- The three-dimensional conservation equations for shell-side flow have been extended to treat the flow of a multicomponent medium. This is necessary because the shell side generally contains a mixture of steam and air. The ability to treat the flow of a multicomponent medium is also essential for assessing the effect of noncondensibles on condenser performance. On the other hand, the computation is simplified by assuming that the steam is at the saturated state and its bulk temperature is determined solely by its partial pressure in the mixture.

- Tube-side fluid flow and heat transfer have been developed and incorporated into the code. The use of one-dimensional conservation equations of mass, momentum, and energy for tube-side flow greatly reduces required computer time without sacrificing accuracy. It also facilitates handling of thermal coupling between the condensation of vapor on the shell side and forced convection of a single-phase liquid on the tube side.

In addition to the foregoing two major additions, several minor modifications and additions have been made, mainly dictated by the consideration of condensation of steam in the presence of noncondensibles. However, it is emphasized that many of the salient features of COMMLX are retained in COMMIX-PPC.

A major unique feature of COMMIX is its porous-medium formulation, which was rigorously derived through local volume averaging. The formulation makes use of the concept of volume porosity, directional surface porosity (a dimensionless vector quantity associated with a surface element), distributed resistance, and distributed heat source or sink. Volume porosity is the ratio of the volume occupied by fluid in a control volume to the total control volume. Surface porosity is similarly defined as the ratio of the area available for fluid flow through a control surface to the total control surface area. Both arise naturally in the averaging process. In the conventional porous-medium formulation, only the volume porosity, distributed resistance, and distributed heat source are used. The concept of directional surface porosity is relatively new. It facilitates the modeling of anisotropic structural resistance to flow. Irregular geometries can also be adapted in the present porous-medium formulation.

The predictive capability of the numerical analysis of fluid flow and heat transfer in complicated engineering systems, e.g., nuclear reactor cores or the shell side of a steam condenser, depends strongly on how well the distributed resistances are modeled. The resistances would vary with their orientation relative to the general flow direction and are oftentimes not known a priori. The directional surface porosity is a geometrical quantity 
and can be accurately and unambiguously calculated. Its introduction lessens the dependency of the velocity field on the modeling of the flow resistance and hence. improves the accuracy of the numerical prediction. This is a major advantage of the present porous-medium formulation.

The finite-volume formulation used in COMMIX for the mass, momentum, and energy conservation equations has recently been extended to include variable-density flows. The modified fornulation employs the concept of a volume-average mass-weighted velocity. As a consequence, the numerical procedure is more robust than the previous COMMLX versions. It also forces the location of pressure change to coincide with that of density change in one-dimensional flows. In addition, the discretized momentum equation also satisfies the one-dimensional Bernoulli equation.

The COMMIX code provides detailed implied velocity and temperature fields for the system under consideration. The conservation equations of mass, momentum, and energy. and the transport equations of the turbulence parameters are solved as boundary-value problems in space and initial-value problems in time. The discretized equations are obtained by integrating the conservation equations over a control volume. The code is flexible and has a wide range of applicability. It is capable of solving thermal-hydraulic problems involving either a single engineering component, such as a rod bundle, reactor plenum, piping system, or heat exchanger, or an engineering system that consists of a combination of these components.

COMMLX uses a fully implicit solution scheme called SIMPLEST-ANL. It is a modificationi of the well-known numerical procedure SIMPLER. The code has a modular structure, and permits the use of either Cartesian or cylindrical coordinate systems. COMMLX-PPC contains physical-property packages for water vapor and liquid water. In addition to these two packages, an option is available for users to input simplified property correlations that are valid in the desired range of applications.

There are three matrix solvers in COMMLX-PPC: the successive overrelaxation method (SOR), the direct matrix inversion method (DMIM), and the preconditioned conjugate gradient method (PCG). SOR and DMIM are suitable for both symmetric and nonsymmetric matrices and are selected for solving the pressure equation and scalar transport equations. PCG is only applicable to a symmetric matrix and thus may be used only for the pressure equation. Depending on the size of the matrix, the user may choose the particular solver that is best suited for the problem in question. With the availability of the three matrix solvers, the efficiency of the numerical computation capability of COMMLX-PPC is greatly increased.

Another unique feature of the COMMLX code is its geometry package. The basic concept is to use computational cells (either in Cartesian or cylindrical coordinates) as building blocks that are stacked to approximate the shape of the physical systems under consideration. Then, volume porosity and directional surface porosity are used to account for differences between the geometry used in the computation and the actual configuration. This feature permits the COMMIX code to model irregular and complex geometries with relative ease. Furthermore, the computer storage requirement of the COMMIX code is optimized; only the computational cells used in the calculations are counted.

Volume I, Equations and Numer/s, of this report describes in detail the basic conservation equations, finite-volume formulation, turbulence modeling. one-dimensional 
treatment of tube-side flow, and solution procedures. Auxiliary models for the following phenomena are also described:

- Momentum interaction between fluid and stationary solid structures.

- Thermal interaction between shell- and tube-side fluid and stationary solid structures.

In II, User's Guide and Manual, we provide flow charts, descriptions of subrotitis geometry modeling, Initialization procedures, input descriptions, etc. A sampile pioblem is included to familiarize readers with the input/output structures of the code. Also included is a sensitivity study of the effects of both condenser inlet air mass fraction and exit mass flow rate at the extraction pipe on steam condensation rate. Wherever applicable, technical content of COMMIX-1C is duplicated here.

\section{Introduction}

\subsection{Purpose}

COMMIX (Component Muing) is a general-purpose computer code for the analysis of heat transfer and fluid flow encountered in real engineering systems. Since the development of COMMIX-1 in 1976, many features have been added and/or refined to augment the code's applicability. Consequently, COMMIX has become a general-purpose computer code with a very wide range of applications. Although developed originally for nuclear-reactor applications, COMMIX can be used, with minor modification, to analyze fluid flow and heat transfer processes in other engineering systems.

Many industries and organizations involved in the design and/or analysis of nuclear reactors are already using COMMIX. However, due to its generalized formulation and the manner in which it is structured. COMMIX has also been found to be a useful tool in other disciplines. Hence, the number of COMMLX users is expected to increase in the future. We have prepared this report keeping in mind prospective users who can benefit from a comprehensive description of the code.

In describing COMMIX-PPC, we had two distinct goals. One was to convey to the reader the capabilities of COMMIX, the equations it solves, and how they are solved: this was done in Volume I of this report. The second goal was to present a step-by-step procedure for the use of COMMIX. Accordingly, the procedure was described in sufficient detail so the usual difficulty of comprehending someone else's code is minimized. This, of course, is not an easy task, but we have attempted it in Volume II.

We stress here that, while extending previous COMMIX versions to COMMIX-PPC, we have tried to retain the original structure and format of COMMLX as much as possible. Therefore, previous COMMIX users will have little difficulty in adapting and running their problems with the COMMIX-PPC version.

Volume I, Equations and Numerics, describes the basic equations, formulations of the discretized equations, auxiliary models, solution procedures, etc. Vol. II. User's Gulde and Manual, describes all information needed by the user, e.g., input description, flow chart. 
solution methods, sample problems, output formats, and user's options. The results of a sensitivity study of the effects of both condenser inlet air mass fraction and exit mass flow rate at the air extraction pipe on steam condensation rate are presented. Wherever applicable, technical content of COMMIX-1C is duplicated here.

\subsection{Organization of the Report}

This volume describes the step-by-step procedure in sufficient detail so that a reader unfamiliar with the COMMLX code may begin using it with minimum difficulty.

An introduction is presented in Sec. 1. Section 2 describes the overall structure of the program, including the flow chart and solution sequence. For a user to start numerical simulation, the first step is to model the geometry. In Sec. 3, we describe the geometrical conventions used in COMMIX, recommend modeling proceriures, and suggest ways to prepare geometry-related input for the code. Section 4 addresses the initialization of the variables, including simplified procedures for shell-side, as well as tube-side, flows, and describes several available boundary condition options.

The modeling of the thermal interaction between a fluid and solid structures is discussed in Sec. 5; that of the momentum interaction between a fluid and the solid structures is given in Sec. 6. The solution procedure is presented in Sec. 7 . In COMMIX-PPC, we have implemented three matrix solvers: the successive overrelaxation (SOR) method, the direct matrix inversion method (DMIM), and the preconditioned conjugate gradient (PCG) method. The user can select a matrix solver, depending on the nature of the problem. The choice of these solvers is discussed in terms of convergence rate and computation efficiency.

Auxiliary inputs, such as turbulence modeling, heat transfer correlations, and physical property determination, are described in Sec. 8.

COMMIX requires initial estimation of the steady-state distribution of all dependent variables regardless of whether the analysis is for a steady-state problem or for a transient simulation. All inputs required for such steady-state calculation are described in Sec. 9. The details related to transient simulations are presented in Sec. 10. In Sec. 11, the input/output procedures and related variables are described. Section 12 discusses global mass and thermal energy balances, and Sec. 13 contains final concluding remarks.

Appendix A summarizes all input variables for convenient reference by the user. Appendix B lists all subroutines, together with a brief description of their functions, and Appendix $\mathrm{C}$ contains a list of resistance correlations.

To illustrate the capabilities of COMMIX-PPC, a sample problem, along with its description, input, and output, is presented in Appendix D. Appendix E presents a sensitivity study of the effect of both condenser inlet air mass fraction and exit mass flow rate at the air extraction pipe on steam condensation rate. 


\section{Structure of COMMIX-PPC}

\subsection{Overall Flow Chart}

The overall structure of the computer program is illustrated by the simplified flow chart presented in Fig. 1, where $\phi$ represents the dependent varlables. The major steps are as follows:

- The numerical simulation begins with grid specification and the calculation of all geometrical quantities that are needed frequently in later work.

Reading of the geometry input is done in the subroutine GEOM3D.

Calculation of all geometrical quantities is done in the subroutine BOXES, for which either the Cartesian or the cylindrical coordinate system may be selected.

- Next. the initial values of all variables are either specified or calculated. The subroutine INITAL is the main subroutine for initialization. It sets up the default values, reads NAMELIST/DATA/, and calls appropriate subroutines (see Table 1 for reading input data, calculating initial values, and printing, if desired, of input and control parameters).

- The tube-side computational cell location cards are read in the subroutine TBINPR, and the geometrical quantities are computed.

- After completion of the initialization, the subroutine OUTPUT is called to print initial values of all desired variables.

- The solution sequence (details of which are given later) is then executed to calculate the values of all dependent variables at a new time. Subroutine TIMSTP determines the sequence of calling subroutines required during the solution sequence. When the values of all variables at the new time are determined, we return to the main subroutine AMAIN.

- If printing is desired at this point, the subroutine OUTPUT is called and the desired variables are printed.

- If the required number of time steps has been performed, or the maximum computation time or the maximum real time is reached, the computation is terminated and, if requested, the restart data are written on disk file. Otherwise, old time values are updated and the execution continues for the next time step until it converges.

\subsection{Solution Sequence}

The solution sequence is controlled by the subroutine TIMSTP; its flow diagram is shown in Fig. 2. Subroutine TIMSTP is the heart of the program; it performs the outer iteration loop. 


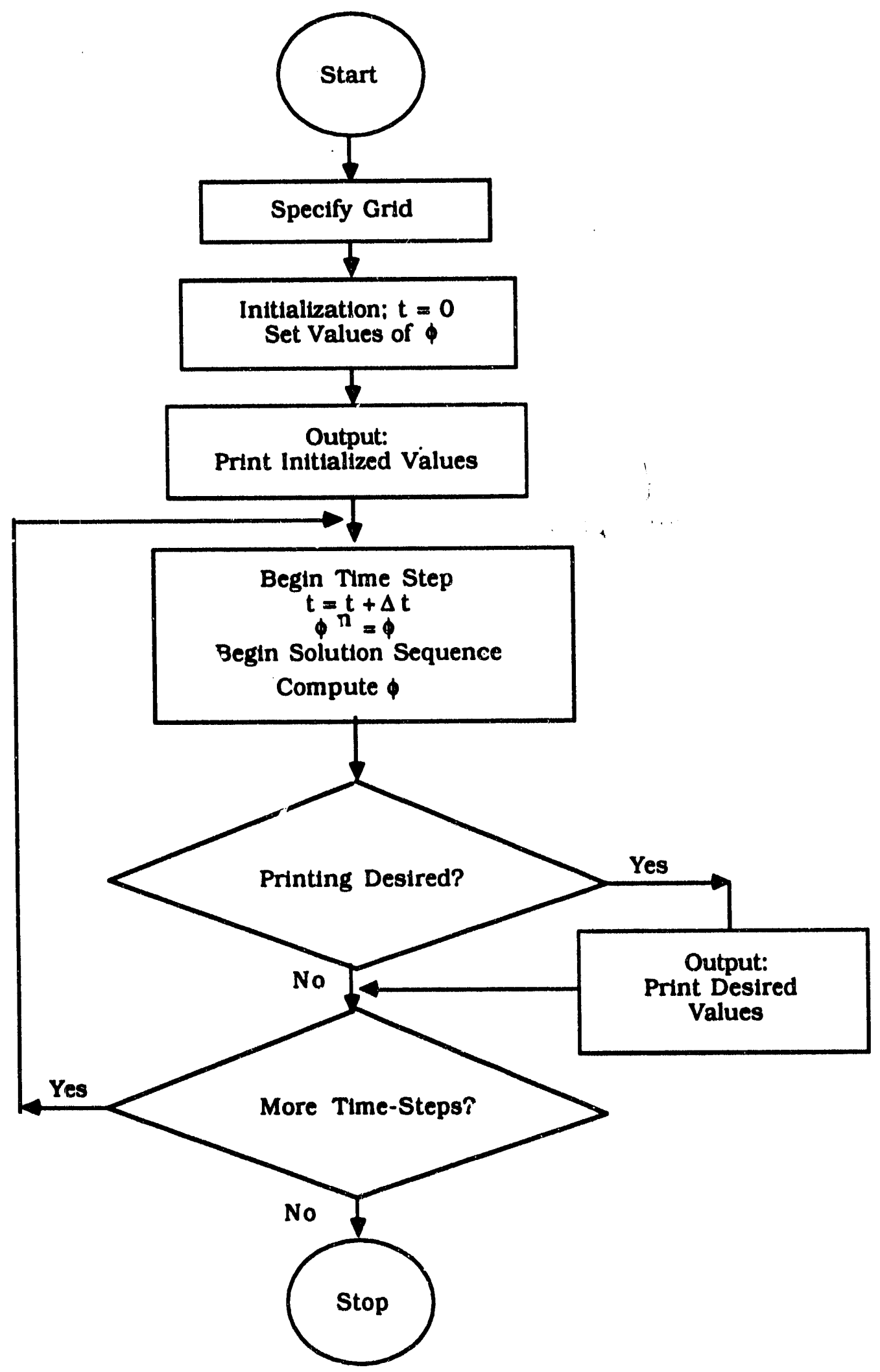

Fig. 1. COMMDX-PPC flow chart 
Table 1. Initializing subroutines

\begin{tabular}{|c|c|c|c|c|}
\hline \multirow{2}{*}{\multicolumn{2}{|c|}{ Subroutine }} & \multicolumn{2}{|c|}{ Input Data } & \multirow{2}{*}{$\begin{array}{l}\text { Function or } \\
\text { Parameters Initialized }\end{array}$} \\
\hline & & NAMELIST & RECORDS & \\
\hline & INITAL & $\begin{array}{l}\text { GEOM } \\
\text { DATA }\end{array}$ & - & $\begin{array}{l}\text { Initializes default values and } \\
\text { calls initializing subroutines }\end{array}$ \\
\hline & INFORC & DATA & Force-structure & Force-structure parameters \\
\hline & INPSTR & $\begin{array}{l}\text { STRUCT } \\
\text { T } \\
\text { F } \\
\text { M }\end{array}$ & $\begin{array}{l}\text { Thermal-structure } \\
\text { prototype and } \\
\text { location records }\end{array}$ & $\begin{array}{l}\text { Thermal-structure } \\
\text { parameters }\end{array}$ \\
\hline & ICTEMP & DATA & - & $\begin{array}{l}\text { Boundary values of velocity, } \\
\text { temperature, heat, density, } \\
\text { and pressure }\end{array}$ \\
\hline & ICTUBE & TUBS & - & $\begin{array}{l}\text { Values of velocity, teinperature, } \\
\text { and pressure for tube-side flow }\end{array}$ \\
\hline$\vdots$ & BARIN & - & $\begin{array}{l}\text { Boundary and } \\
\text { initial-value } \\
\text { records }\end{array}$ & $\begin{array}{l}\text { Boundary and initial values of } \\
\text { pressure, velocity, tempera- } \\
\text { ture, enthalpy, heat source, } \\
\text { porosity, and surface area }\end{array}$ \\
\hline
\end{tabular}

The six major loops in subroutine TIMSTP are

- Shell-Side and Tube-Side Heat Transfer Coupling Loop: Heat transfer coefficients, heat transfer rates from steam to tube and from tube to cooling water, and steam condensation rate are calculated. Subroutine HSTRUC computes the heat transfer coefficients, subroutine QSTRUC computes the heat transfer rates both in shell side and tube side, and subroutine INMDOT computes the steam condensation rate.

- Mass-Momentum Loop: Mass and momentum equations are solved and new velocity and pressure values are computed. Subroutine MOLOOP calls the required subroutines in proper sequence to solve the mass and momentum equations.

- Turbulence LoOp: Two subroutines, TKLOOP and TDLOOP, are called to calculate the turbulense parameters $k$ (turbulence kinetic energy) and $\varepsilon$ (rate of dissipation of $\mathbf{k}$ ). Turbulent viscosity and conductivity are then calculated by subroutine TURV12. 


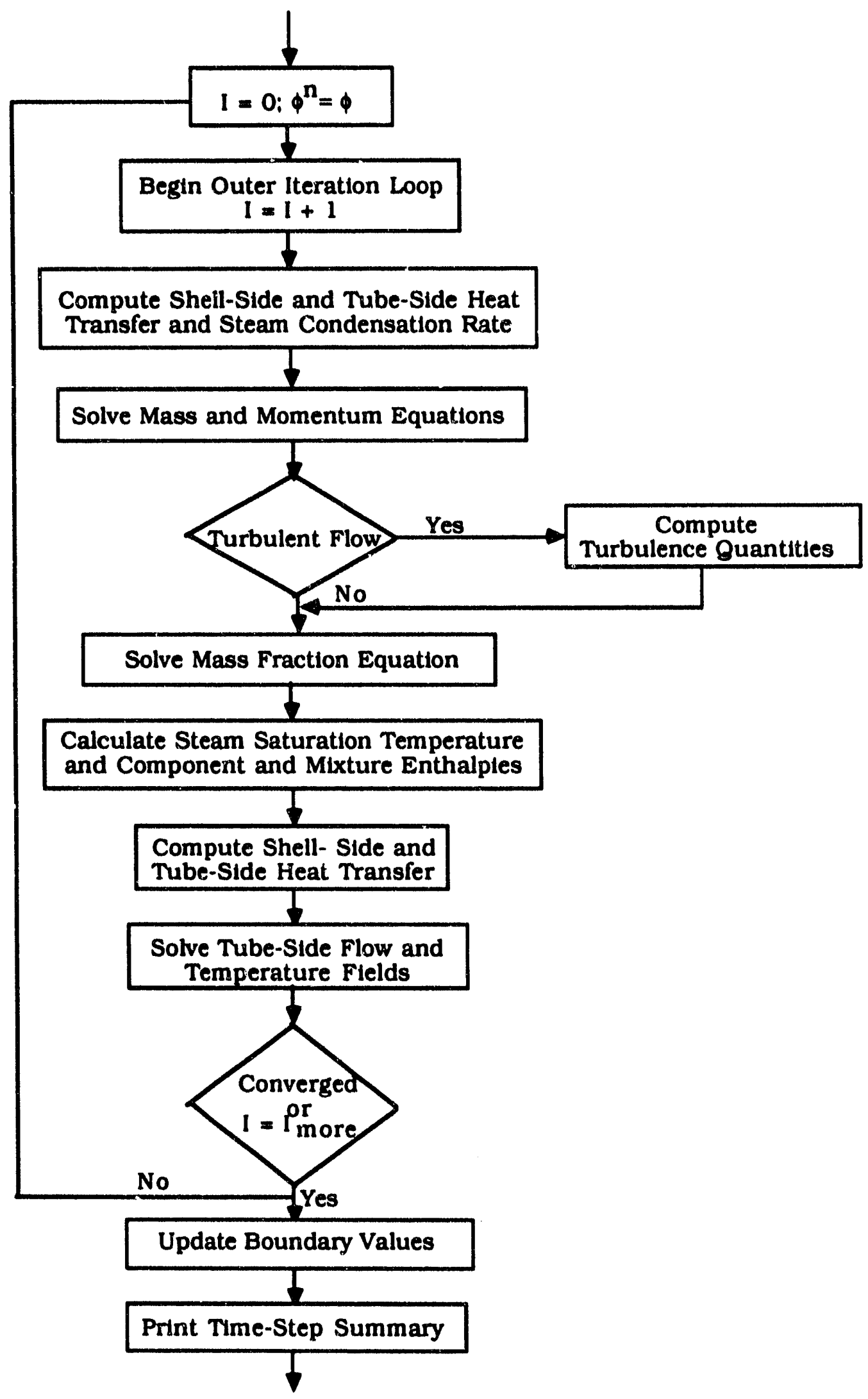

Fig. 2. Flow chart for solution sequence in subroutine TIMSTP 
- Component Mass Fraction Loop: Component continuity equations are solved and new values of component mass fraction are calculated. Subroutine MALOOP calls the required subroutines in sequence to solve the component mass conservation equation.

- Energy Loop: The saturation temperature of steam is calculated from the steam partial pressure. Subroutine ENLOOP computes the saturation temperature of steam. New values of enthalpies of steam, air, and a stearnair mixture are computed.

- Tube-Side Flow and Temperature Loop: The one-dimensional tube-side flow and temperature fields are solved and new values are calculated for temperature, pressure, and velocity. Subroutine TBSIDE solves the one-dimensional continuity, momentum, and energy equations.

\subsection{Shell- and Tube-Side Heat Transfer Coupling Loop}

The flow chart for the shell- and tube-side heat transfer coupling loop is shown in Fig. 3. The calling sequence is as follows:

- First, calculate the heat transfer coefficients $h_{a}, h_{c}$, and $h_{c w}$ according to Eqs. 9.3, 9.4, and 9.14 of Vol. I, respectively, and the fouling resistance $R_{f}$ described in Eq. 9.35 also of Vol. I. These quantities are then used to compute the shell-side and tube-side heat transfer coefficients $h_{1}$ and $h_{2}$ according to Eqs. 9.32 and 9.41 of Vol. I, respectively. The calculations of $h_{a}, h_{c}, h_{c w}, R_{f}, h_{1}$, and $h_{2}$ are performed in subroutines HSTRUC, HTCOEF, HTCOND, and HTCTUB.

- Use heat transfer coefficients $h_{1}$ and $h_{2}$. tube geometrical information, material properties, and the steam/air temperature to calculate coefficients $A_{\ell}^{\prime}$ and $C_{\ell}$ for $\ell=1-L$, as described in Eqs. 9.51-9.54. Then, solve for temperature distribution $T_{l}(l=1 \ldots L)$ from Eqs. 9.48-9.50, and compute the heat transfer rate $\dot{q}_{1}$ and $\dot{q}_{L+1}$ from Eqs. 9.55 and 9.56, all of Vol. 1. These calculations are performed by subroutine QSTRUC.

- Use $\dot{q}_{1}$ and latent heat to calculate the steam condensation rate $\dot{m}$ by subroutine INMDOT.

The flow diagrams for the mass-momentum, component mass fraction, energy, and tubeside flow and temperature loops are presented in Secs. 2.4, 2.5, 2.6, and 2.7, respectively. The turbulence loop is discussed in Sec. 8.3.

\subsection{Mass-Momentum Loop}

The flow chart for the mass-momentum loop is shown in Fig. 4. The calling sequence is performed by subroutine MOLOOP. 


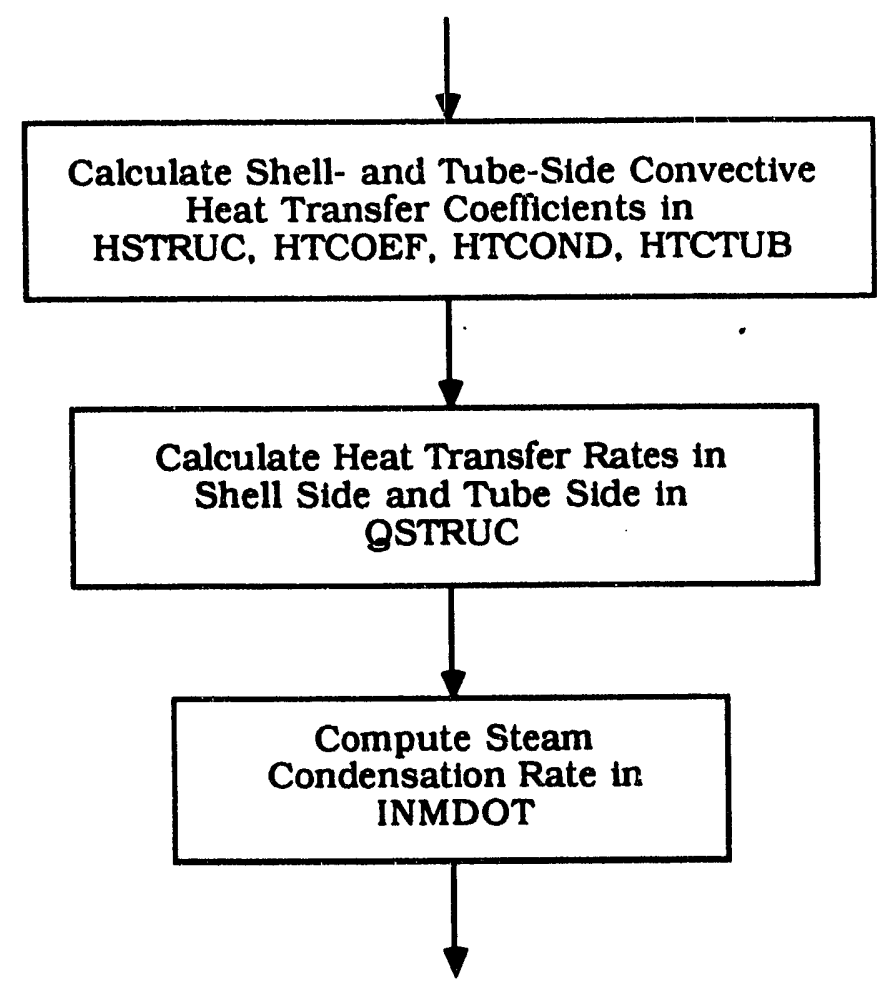

Fig. 3. Flow diagram for shell- and tube-side heat transfer coupling loop

- First, calculate the coefficients $\hat{u}, \hat{v}, \hat{w}, d u, d v$, and $d w$ in the pressurevelocity relation described in Secs. 5 and 6 of Vol. $i$. These calculations are performed in subroutines XMOMI. YMOMI, and ZMOMI for $x$ (or r), y (or $\theta$ ), and $\mathrm{z}$ directions, respectively.

- Use these pressure-velocity-relation coefficients to calculate the coeíicients $a_{0}^{p}, a_{1}^{p}, \ldots . a_{6}^{p}, b_{0}^{p}$ (see Table 12 of Vol. I) in the pressure equation. This is done by subroutine PEQN.

- The pressure equation is then solved in subroutine SOLVER by one of the matrix solvers (subroutine SOLVIT for the SOR method, subroutine DMIM/for the DMIM, and subroutine CGRAD for the PCG method). If the SOR or PCG method is selected by the user, the solution is obtained by an iterative procedure.

The iteration is continued until either the residue of the pressure equation is below the specified convergence criterion or the number of iterations has reached the specified maximum value, called ITMAXP. If the DMIM is selected, the solution is obtained by direct inversion and no iteration is involved.

- Velocities are then updated in subroutine MOMENI with the new pressure values and the following relations: 


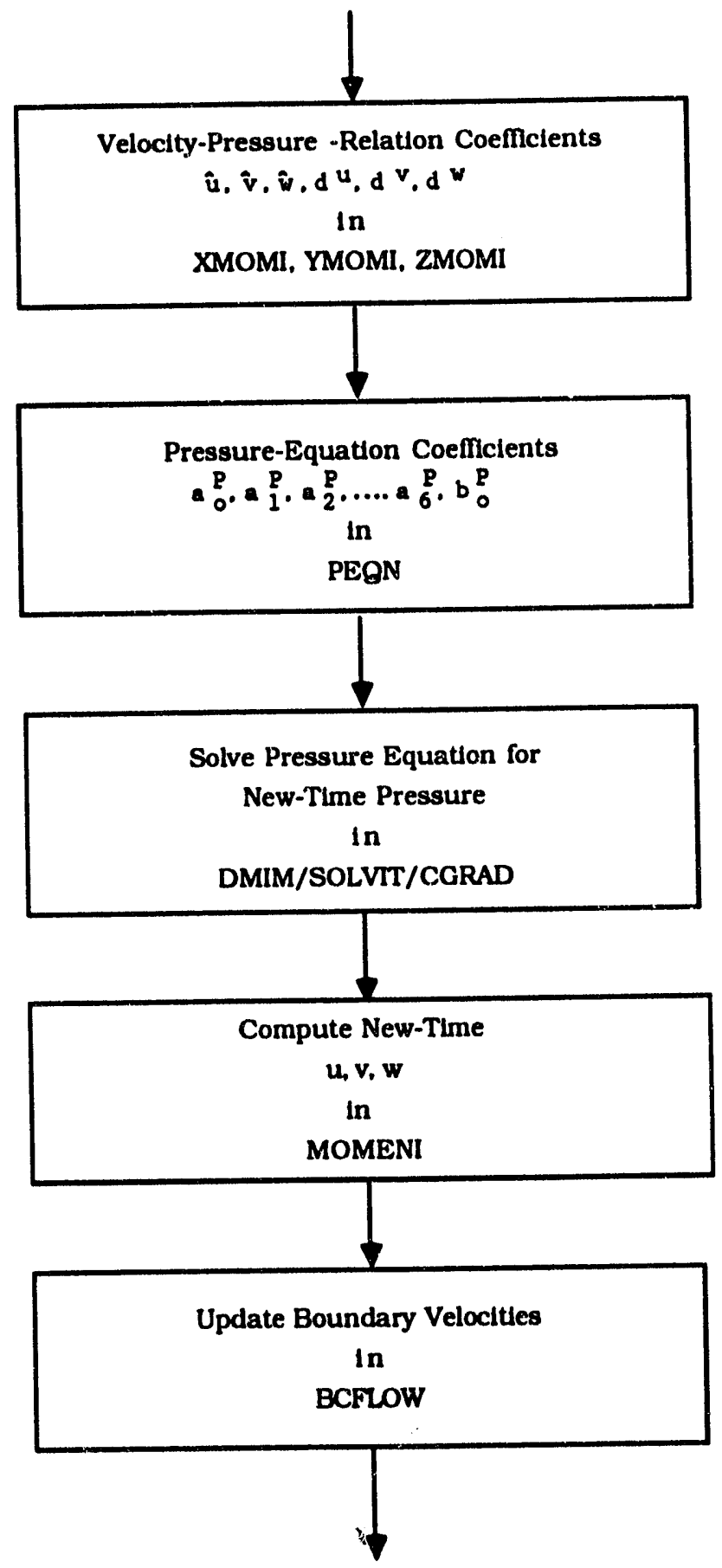

Fig. 4. Flow diagram for mass-momentum loop 


$$
\begin{aligned}
& u=\hat{u}-d^{u}\left(\delta p_{2}-\delta p_{0}\right) \\
& v=\hat{v}-d^{v}\left(\delta p_{4}-\delta p_{0}\right)
\end{aligned}
$$

and

$$
w=\hat{w}-d^{w}\left(\delta p_{\theta}-\delta p_{0}\right)
$$

- Finally, update the velocity boundary values in subroutine BCFLOW.

This completes the mass-momentum loop.

\subsection{Component Mass Fraction Loop}

The sequence of computations in the component mass fraction loop (called by subroutine MALOOP) is shown in Fig. 5.

- Compute the convection terms in subroutine MACONØ.

- Compute the diffusion term in subroutine MADIFF.

- Compute the trarisient and source terms in subroutine MASSCO.

- Solve the mass fraction equations

$$
\left(a_{0}^{x} x_{0}^{n+1}\right)_{1}=\sum_{l}\left(a_{l}^{x} x_{l}^{n+1}\right)_{1}+\left(b_{0}^{x}\right)_{1} \quad(i=2.3, \ldots n)
$$

by subroutir a SOLVER for the "new-time mass fractions by either the SOR method (subroutine SOLVIT) or the DMIM (subroutine DMIM). Note that 1 indicates the component number.

- Compute the mass fraction of the first component by the following relation:

$$
x_{1}=1-\sum_{i=2}^{n} x_{1}
$$

\subsection{Energy Loop}

The sequence of computations in the energy loop (called by subroutine ENLOOP) is shown in Fig. 6.

- Update all boundary values in subroutine BCTENT.

- Compute the steam partial pressure.

- Compute the steam saturation temperature based on steam partial pressure.

- Compute the enthalples of steam, air, and steam and air mixture. 


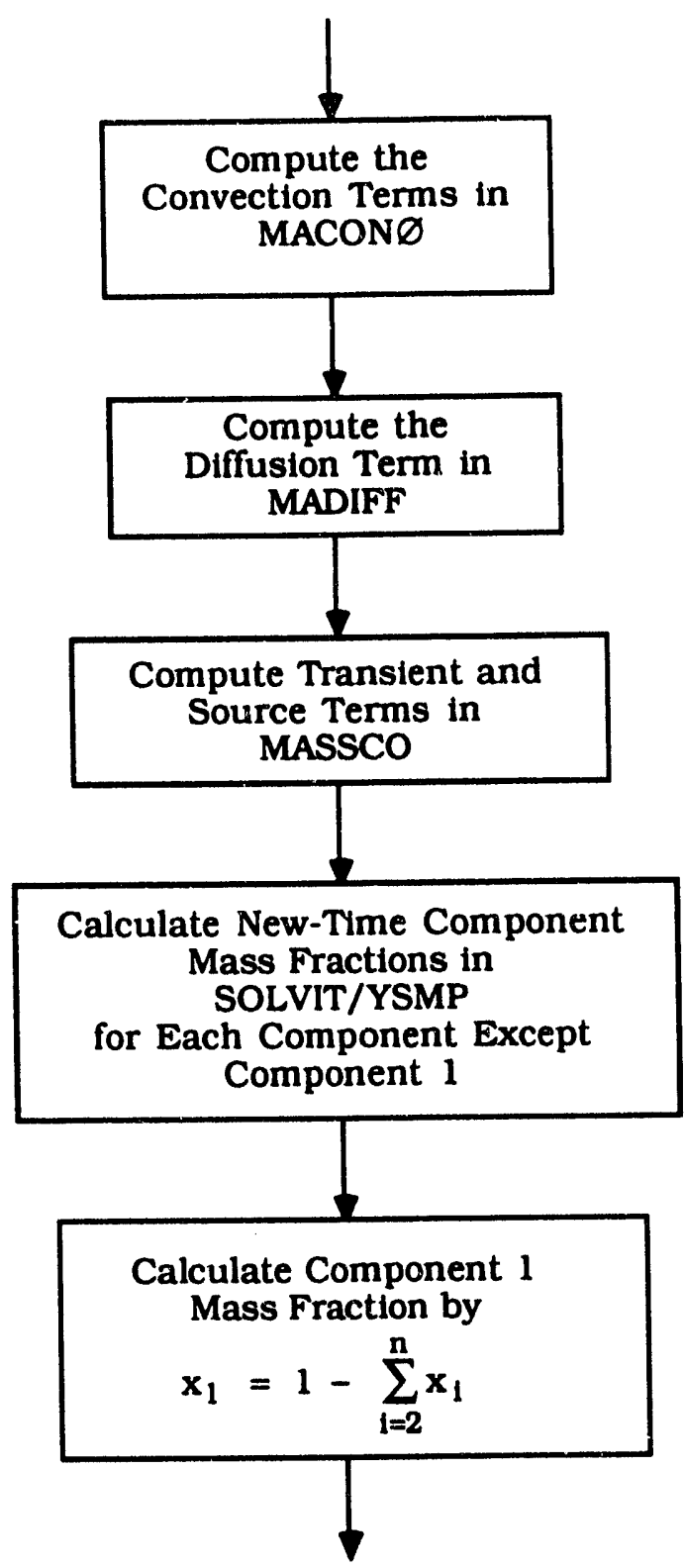

Fig. 5. Flow diagram for component mass fraction loop

\subsection{Tubo-Side Flow and Temperature Loop}

The sequence of computations in the tube-side loop (called by subroutine TBSIDE) is shown in Fig. 7.

- Compute coefficients in the velocity-pressure relation.

- Compute coefficients in the pressure equation.

- Solve pressure equation for the new-time pressure.

- Compute new-time velocity. 


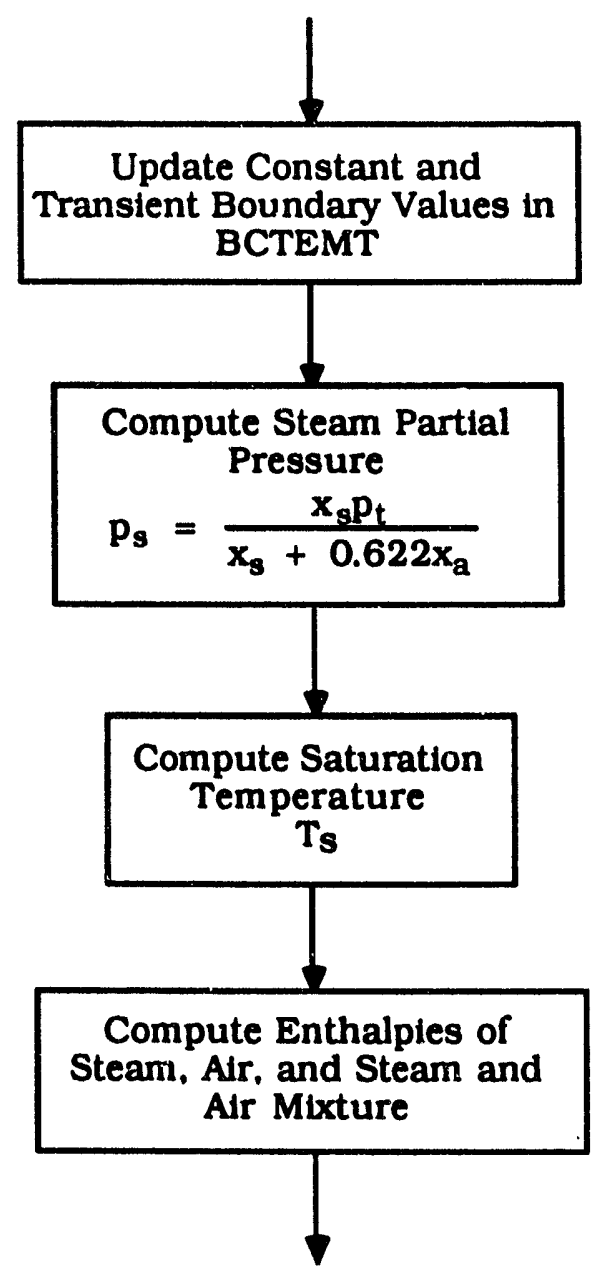

Fig. 6. Flow diagram for energy loop

- Solve energy equation for new-time temperature and enthalpy.

- Update boundary velocities and temperature.

\section{Geometry Modeling}

\subsection{Introduction}

In most cases, the first step is to model the geometry of the system to be simulated. The geometry concept of COMMIX is somewhat unique and is different from the conventional approach. We hope that, after using the COMMLX geometry package, the user will not only feel at ease with the COMMIX concept, but will begin to appreciate the benefits of the COMMIX approach. To make the introductory period as easy and smooth as possible, we suggest that all new users read this section with extra thoroughness. 


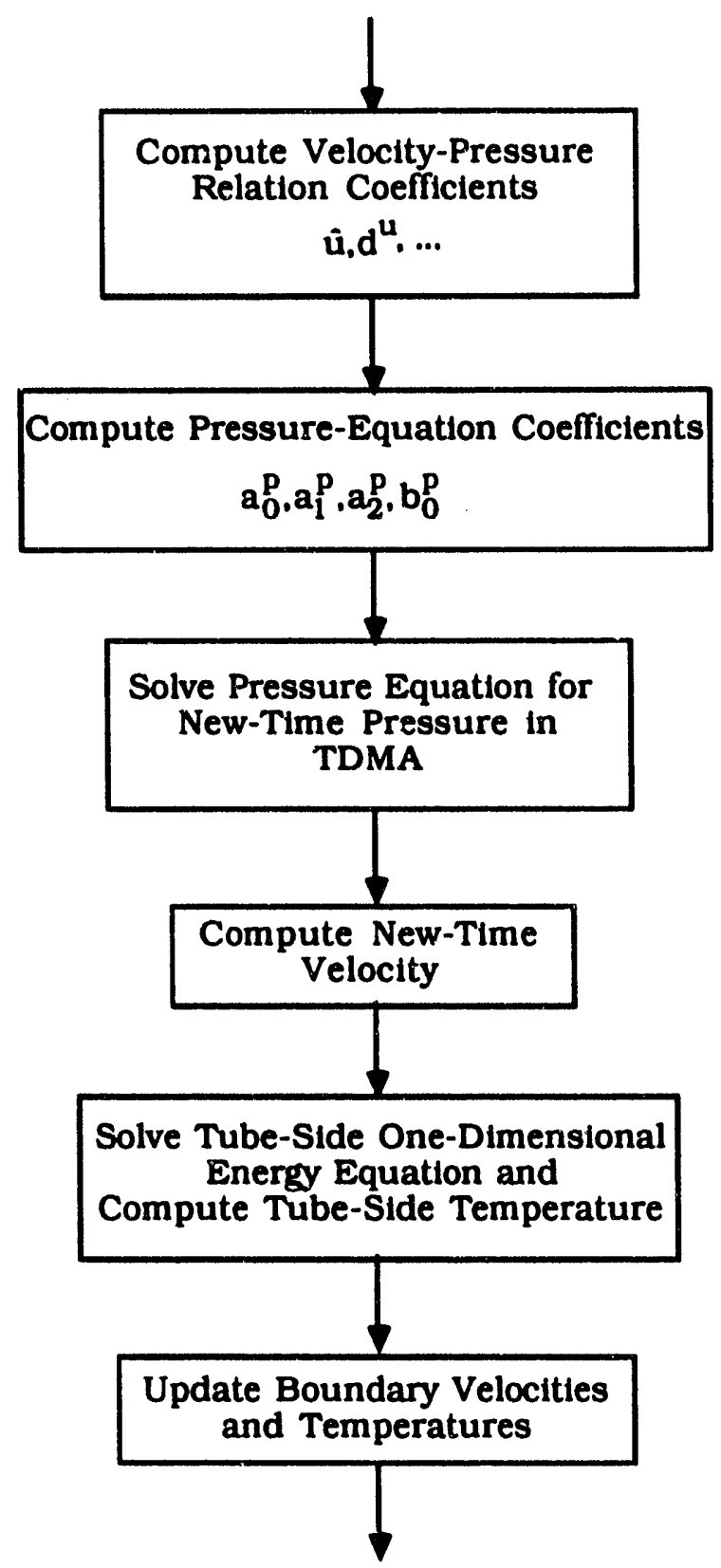

Fig. 7. Flow diagram for tube-side loop

\subsection{Concept and Definitions}

In numerical modeling. the computational flow domain is partitioned into several computational cells formed by $x, y$, and $z$ grid planes in a Cartesian coordinate system or by $r, \theta$, and $z$ grid surfaces in a cylindrical system, as shown in Fig. 8. In COMMLX, the following convention is used to define the various elements of the flow domain. For the purpose of illustration, we consider a simple box geometry shown in Fig. 9. 


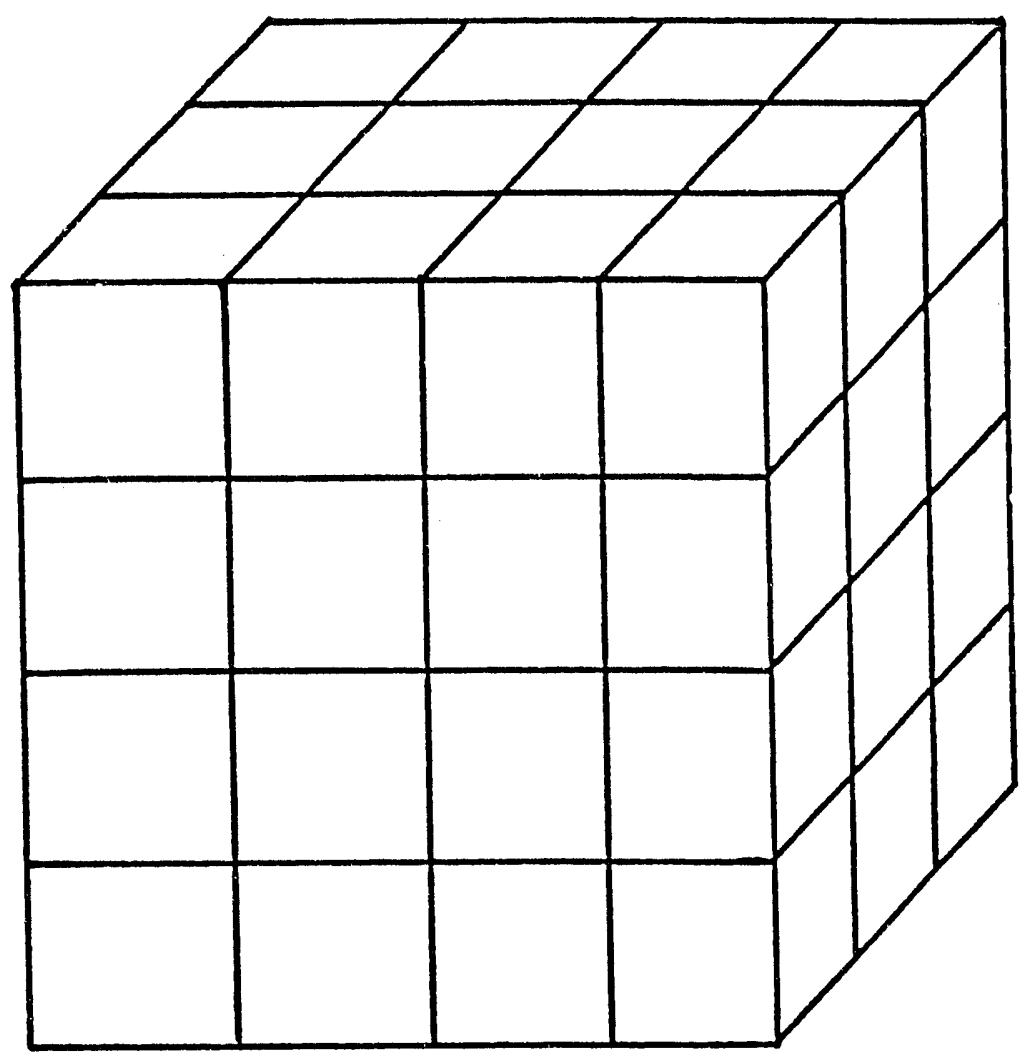

(a)

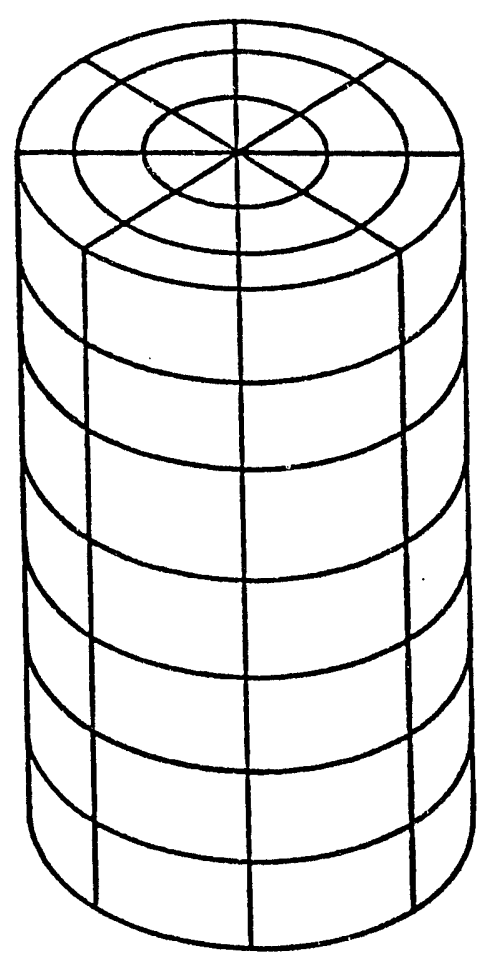

(b)

Fig. 8. Partitioning in (a) Cartesian and (b) cylindrical coordinate systems 

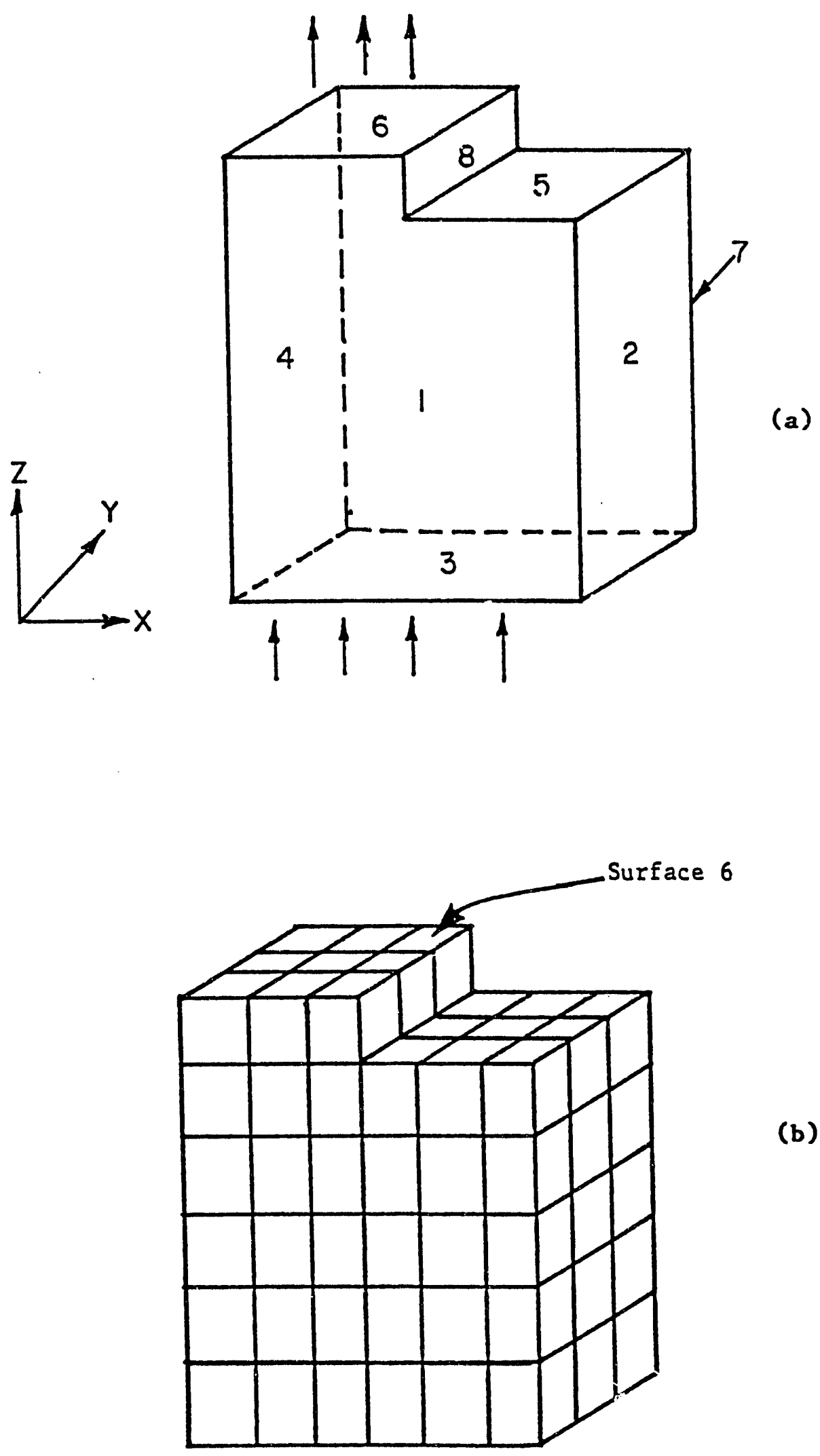

(b)

Fig. 9. Model geometry showing (a) boundary surfaces and (b) partitioning of surface elements 
- Computational Cell

In COMMLX, the computational cell is defined by the location of the grid point place: in the geometrical center of the cell. Cell sizes can be nonuniform. A computational cell surrounded only by other computational cells is called an internal cell. If one cell surface is a boundary surface, it is called a boundary cell. A user must specify the total number of computational cells required through the FORIRAN variable NM1.

\section{, Boundary Surface}

The external boundarles enveloping the flow domain are called the boundary surfaces. These may be solid walls or planes through which fluid can flow. The geometry in Fig. 9a has eight boundary surfaces.

A boundary surface is defined by its unit normal vector, always pointing into the fluid region. The $x, y$, and $z$ components (XNORML. YNORML, and ZNORML) of a unit normal vector are specified by its direction cosines. For example, the unit normal vector of Surface 2 has components $(-1,0,0)$. Table 2 lists the components of the unit normal vectors of the eight surfaces of the flow domain shown in Fig. 9a.

\section{- Regular Surface}

A boundary surface coinciding with any one of the grid planes is called a regular surface. All surfaces of the geometry of Fig. 9 are regular surfaces.

Table 2. Components of unit normal vectors (direction cosines) of geometry in Fig. $9 a$

\begin{tabular}{cccc}
\hline Surface & XNORML & YNORML & ZNORML \\
\hline 1 & 0 & 1 & 0 \\
2 & -1 & 0 & 0 \\
3 & 0 & 0 & 1 \\
4 & 1 & 0 & 0 \\
5 & 0 & 0 & -1 \\
6 & 0 & 0 & -1 \\
7 & 0 & -1 & 0 \\
8 & -1 & 0 & 0 \\
\hline
\end{tabular}


- Irregular Surface

A boundary surface that does not coincide with any one of the grid planes is called an irregular surface. In Fig. 10a. Surface 1 is an irregular surface. Note that only regular surfaces can be specified as inlet and outlet planes, i.e., flow inlet and outlet planes cannot be irregular surfaces.

- Surface Number

A user must assign a number to each boundary surface. The same surface number can be assigned to boundary surfaces that have

-the same unit normal vector, and

-the same velocity, pressure, temperature, and heat flux boundary conditions.

For example, although Surfaces 2 and 8 in Fig. 9a are in two different planes, they can be assigned the sarne surface number if their unit normal vector has the same direction cosines and the same boundary conditions, as illustrated in Fig. 11. However, they may also be defined as two different surfaces, if desired.

Surfaces 3 and 8 lie in the same plane, as tllustrated in Fig. 12, and they have the same unit vector. They must be considered as two different surfaces if they have different boundary conditions for velocity or pressure or temperature or heat flux.

The variable NSURF is used to specify a total number of surfaces. All irregular surfaces must be numbered before other regular surfaces are numbered.

- Irregular Cell

If one surface of a computational cell is an irregular surface, that cell is called an irregular cell. An irregular cell cannot be an interior cell because it contains an irregular surface that is part of a boundary surface. A computational cell is permitted to have at most one irregular surface.

- Surface Element

A section of a surface between two pairs of consecutive grid lines is defined as a surface element. A surface can therefore have more than one surface element. For example, in Fig. 9b. Surface 6 has nine surface elements. The variable NL1 is used to specify the tot. number of surface elements.

- Volume Porosity

The volume porosity $\gamma$ is a geometrical parameter that is defined as the ratio of fluid volume in a cell to the total cell volume. The FORTRAN variable name for volume porosity is AL and its numerical value must lie between 0 and 1 . This parameter is included to account for irregularity in geometry and the 

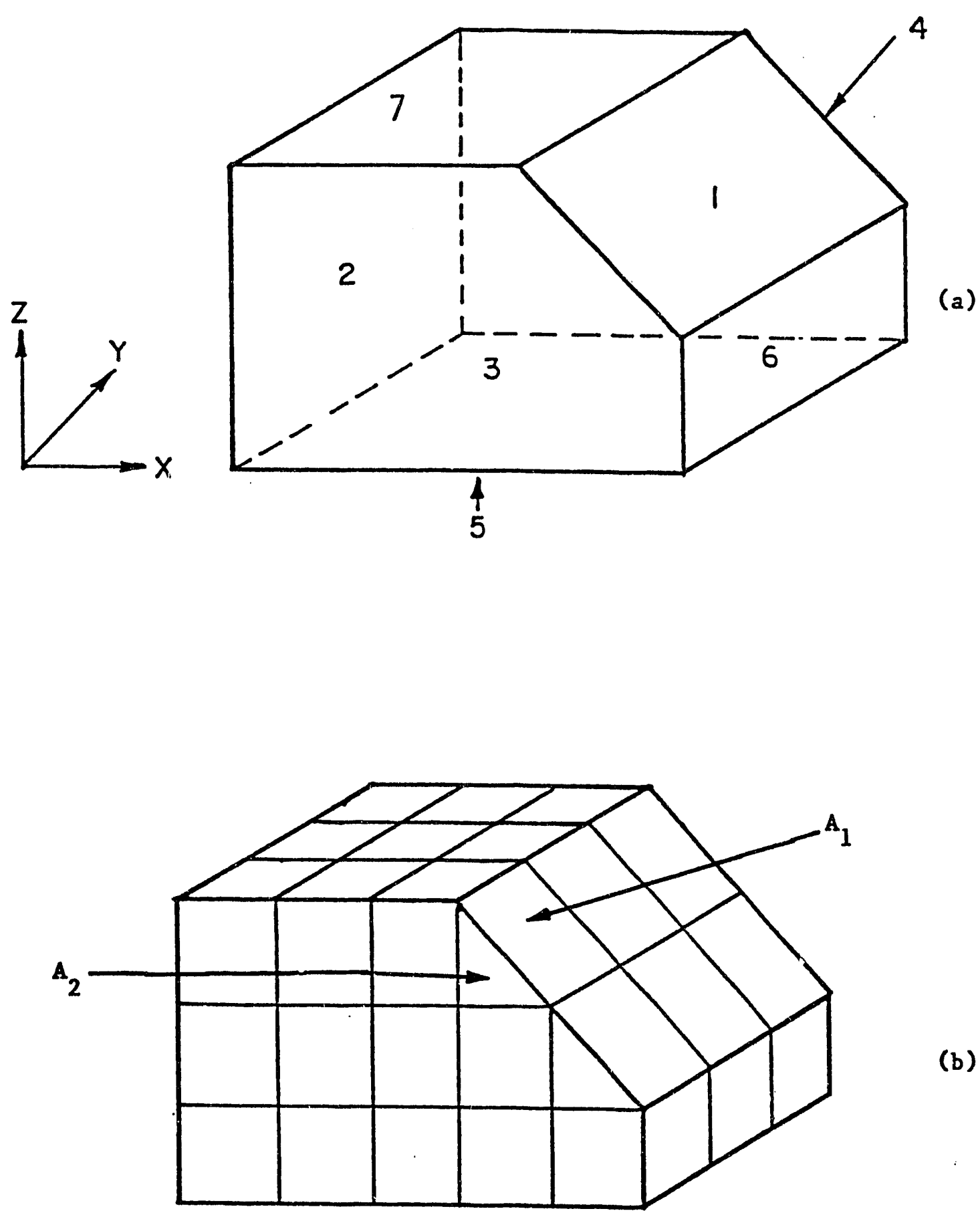

(b)

Fig. 10. Model geometry showing (a) irregular surface and (b) partitioning of surface elements 


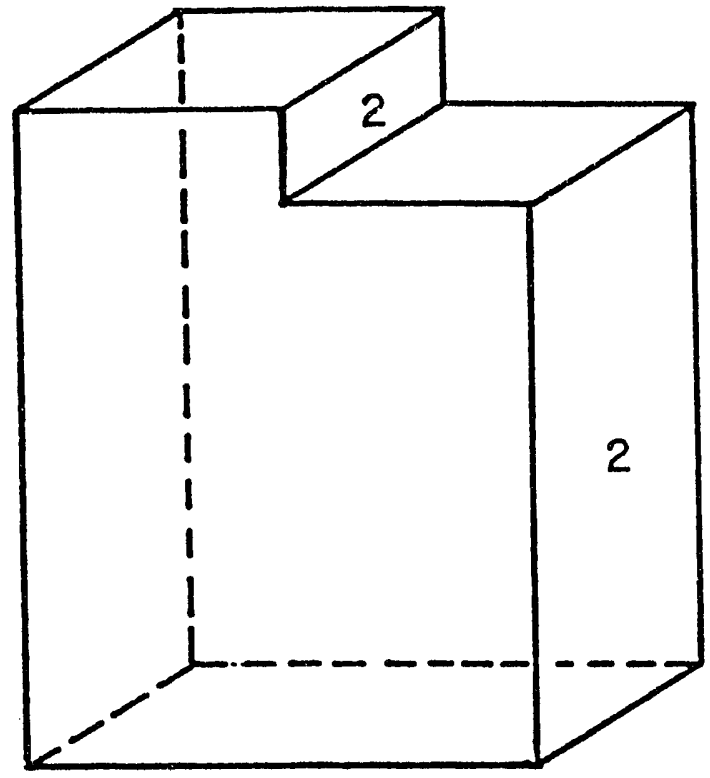

Fig. 11. Two noncoincident surfaces with the same surface number

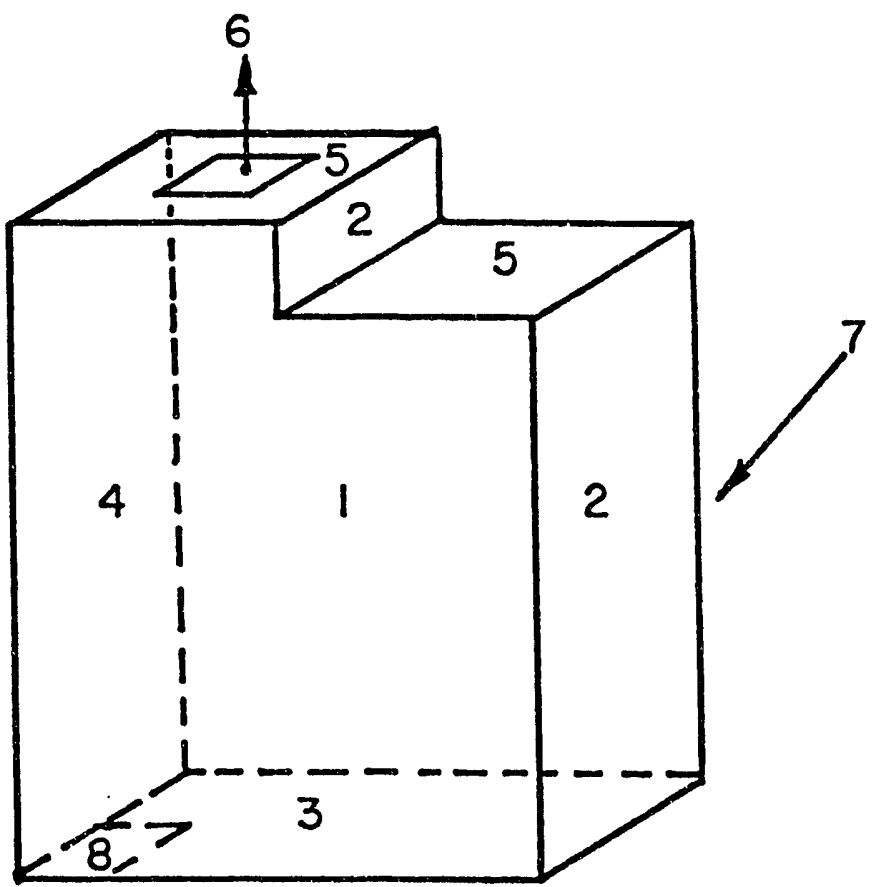

Fig. 12. Two coincident surfaces with different surface numbers 
existence of internal solid structures. For example, the volume porosity of the computational ceil due to irregular geometry (Fig. 13a) is

$$
\gamma_{v}=A L=\frac{\Delta x \Delta y \Delta z-\text { volume of prism } A}{\Delta x \Delta y \Delta z} .
$$

Similarly, the volume porosity due to an internal solid structure (Fig. 13b) is

$$
\gamma_{v}=A L=\frac{\Delta x \Delta y \Delta z-\text { volume of the half solid cylinder }}{\Delta x \Delta y \Delta z} .
$$

- Directional Surface Porosity

The directional surface porosity $\gamma_{x}$ associated with a surface whose normal vector is in the $x$-direction is defined as the ratio of fluid flow area in that surface to total surface area. The directional surface porosity $\gamma_{y}$ and $\gamma_{z}$ can be similarly defined. The variable names are AiX, ALY, and ALZ for the $x$ (or r), $y$ (or $\theta$ ), and $z$ directions, respectively. Thus, in Fig. 13a.

$$
\begin{aligned}
& \gamma_{x}(i+1 / 2, j, k)=A L X(\text { cell } \mathbf{j j k})=(\Delta y \Delta z-\operatorname{area} A 1) /(\Delta y \Delta z) \\
& \gamma_{y}(i, j+1 / 2, k)=A L Y(\text { cell } \mathbf{j j k})=1.0, \text { and } \\
& \gamma_{z}(1, j, k+1 / 2)=A L Z(\text { cell } \mathbf{j j k})=(\Delta x \Delta y-\operatorname{area} A 3) /(\Delta x \Delta y)
\end{aligned}
$$

and in Fig. 13b.

$$
\begin{aligned}
& \gamma_{x}(1+1 / 2, j, k)=A L X(\text { cell } \mathbf{j} k)=(\Delta y \Delta z-\operatorname{area} A 5) /(\Delta y \Delta z) \\
& \gamma_{y}(1, j+1 / 2, k)=A L Y(\text { cell } \mathbf{j} k)=1.0, \text { and } \\
& \gamma_{z}(1, j, k+1 / 2)=A L Z(\text { cell } \mathbf{j} k)=(\Delta x \Delta y-\operatorname{area} A 4) /(\Delta x \Delta y)
\end{aligned}
$$

The directional surface porosity is considered a flow-variable parameter. In the staggered-grid arrangement. the flow-variable parameters are defined at the face of a cell. Therefore, when we describe $\gamma_{x}, \gamma_{y}$, and $\gamma_{z}$ of cell (1,j,k), we mean $\gamma_{x}$ at $(i+1 / 2, j, k), \gamma_{y}$ at $(1, j+1 / 2, k)$, and $\gamma_{z}$ at $(i, j, k+1 / 2)$.

\section{- Tube Channel}

In COMMIX-PPC, a tube channel is defined as a series of computational cells that always contain the same group of condenser tubes. Each channel is assigned a channel number and may contain more than one tube. Thus, in Fig. 14. Channel 1 contains three condenser tubes and Channel 2 contains five condenser tubes. 

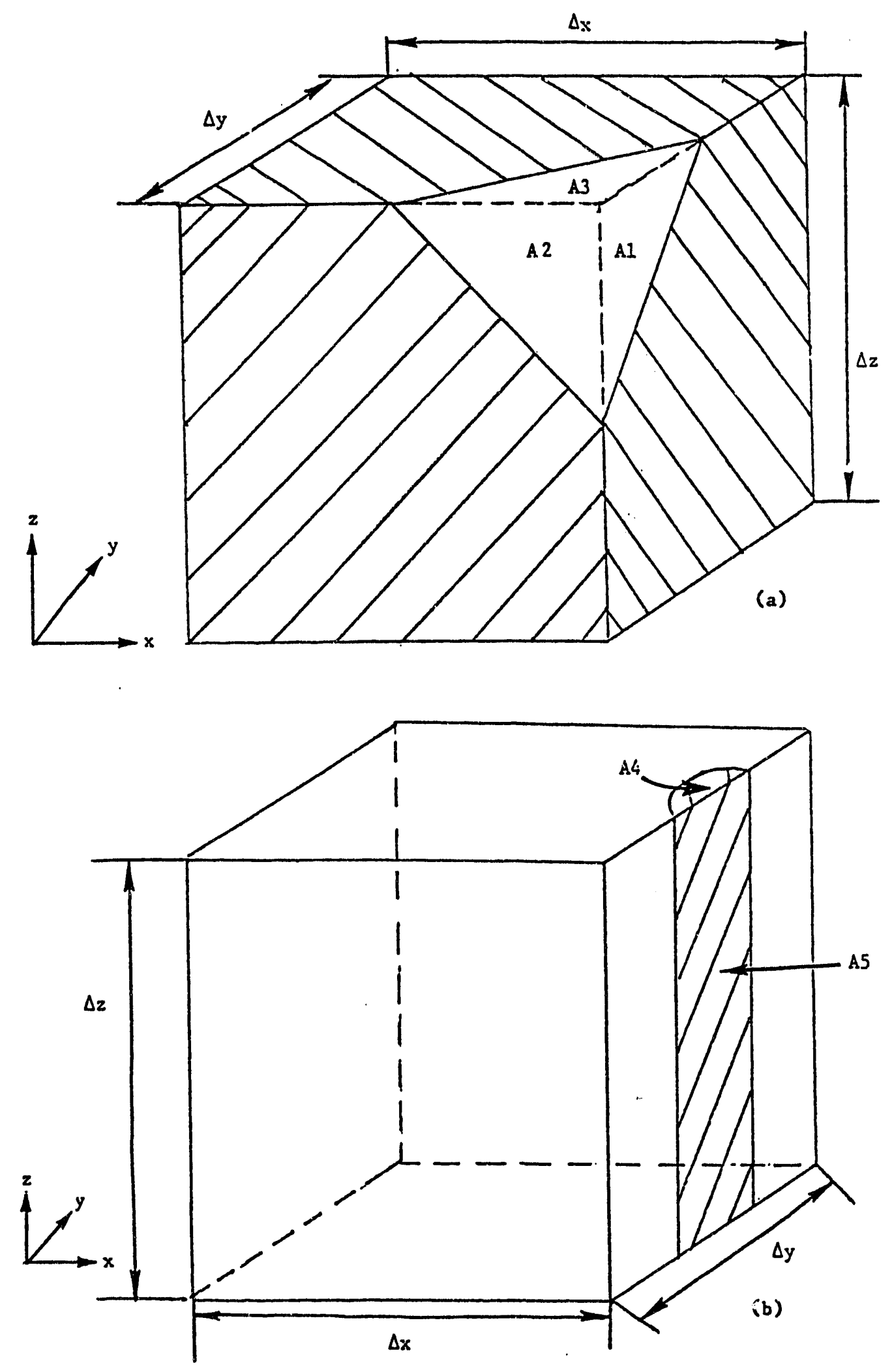

Fig. 13. Volume porosity due to (a) irregular geometry and (b) internal solid structure 


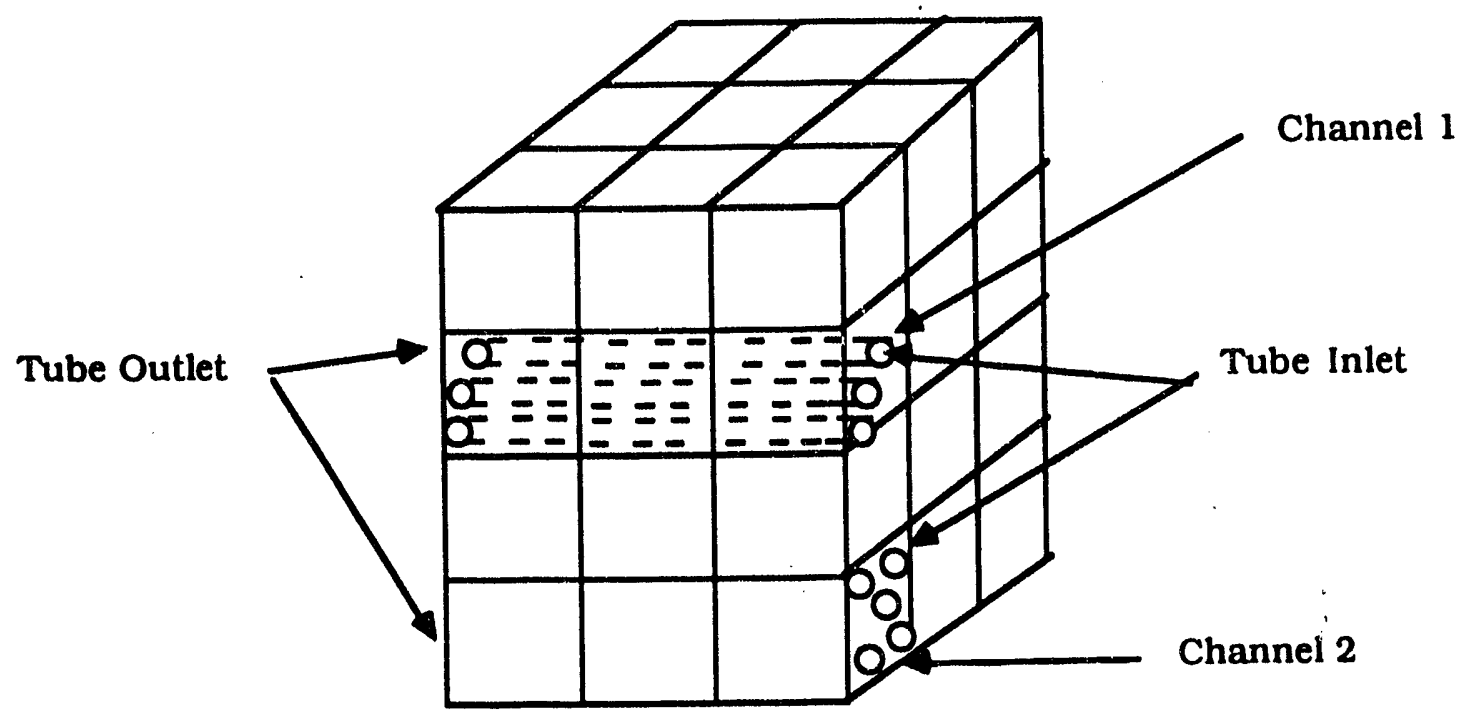

Fig. 14. Distribution of tube channels

\subsection{Recommended Approach}

The task of developing a geometrical model of a flow domain involves

- selection of an appropriate coordinate system.

- partitioning of a computational flow domain into several computational cells bounded by coordinate grid planes or grid surfaces, and

- computation of geometrical partition sizes.

The development of a geometrical model of a physical situation requires a judicious balance between attempting to represent the geometry as accurately as possible on the one hand, and minimizing the computational cost in terms of time and storage on the other. The computational cost generally places an upper limit on the number of computational cells; hence, the model should seek a proper balance between homogenization of the details and resolution and accuracy of the results.

Developing a model and preparing input for a complex geometry involve many decisions. There is no simple, rule-of-thumb procedure in modeling, because most of the decisions that must be made are problem-dependent. The user must seek his/her own intuition and judgment. The following are only a few broad suggestions:

- Select the appropriate coordinate system.

- Take advantage of symmetry and model the system with minimal required geometry. 
- Use a relatively coarse mesh at the beginning to ensure that the input is correct and the results are reasonable. The mesh size should then be reduced to determine its effect on the accuracy of the solution.

- Use fine meshes only in those regions where large variations in parameters or properties are expected to occur.

\subsection{Geometry Input}

When all major elements of geometry modeling are decided, we can start the preparation of geometry input. The following is a recommended step-by-step procedure.

- Specify value of the variable IGEOM.

$$
\begin{aligned}
\text { IGEOM } & =0 & & \text { for Cartesian geometry and } \\
& =-1 & & \text { for cylindrical geometry. }
\end{aligned}
$$

- Partition the flow domain to be modeled by $x, y$, and $z$, or $r, \theta$, and $z$ grid surfaces. Two examples are illustrated in Fig. 15. The following must be specified:

IMAX, JMAX, and KMAX: Maximum number of cells in $x, y$, and $z$ (or $r, \theta$, and 2 ) directions.

DX, DY, and DZ: Partitioning distances in $x, y$, and $z$ directions.

NSURF: Total number of surfaces.

NM 1: Total number of computational cells.

NL1: Total number of surface elements.

- Be sure to take advantage of parameters IFX, IFY, and IFZ for one- and twodimensional calculations. For example, if the system variables are independent of the $x$ (or $r$ for cylindrical coordinate) direction, set IFX $=0$. The code will bypass all calculations that are $x$ (or $r$ ) dependent.

- Assign surface numbers to all boundary surfaces. Compute and specify XNORML, YNORML, and ZNORML (the components of unit normal vectors) for all surfaces. Number all irregular surfaces first.

Partitioning must be done so that each cell has a maximum of one irregular surface.

- Compute areas of (1) surface elements of all irregular surfaces (such as surface area $A_{1}$ in Fig. 10b), and (2) partially truncated regular surface elements of irregular cells (such as surface area $A_{2}$ in Fig. 10b). Prepare and supply all information about surface elements, as described in the section on Boundary Surface Identification Records in the Input Description. 

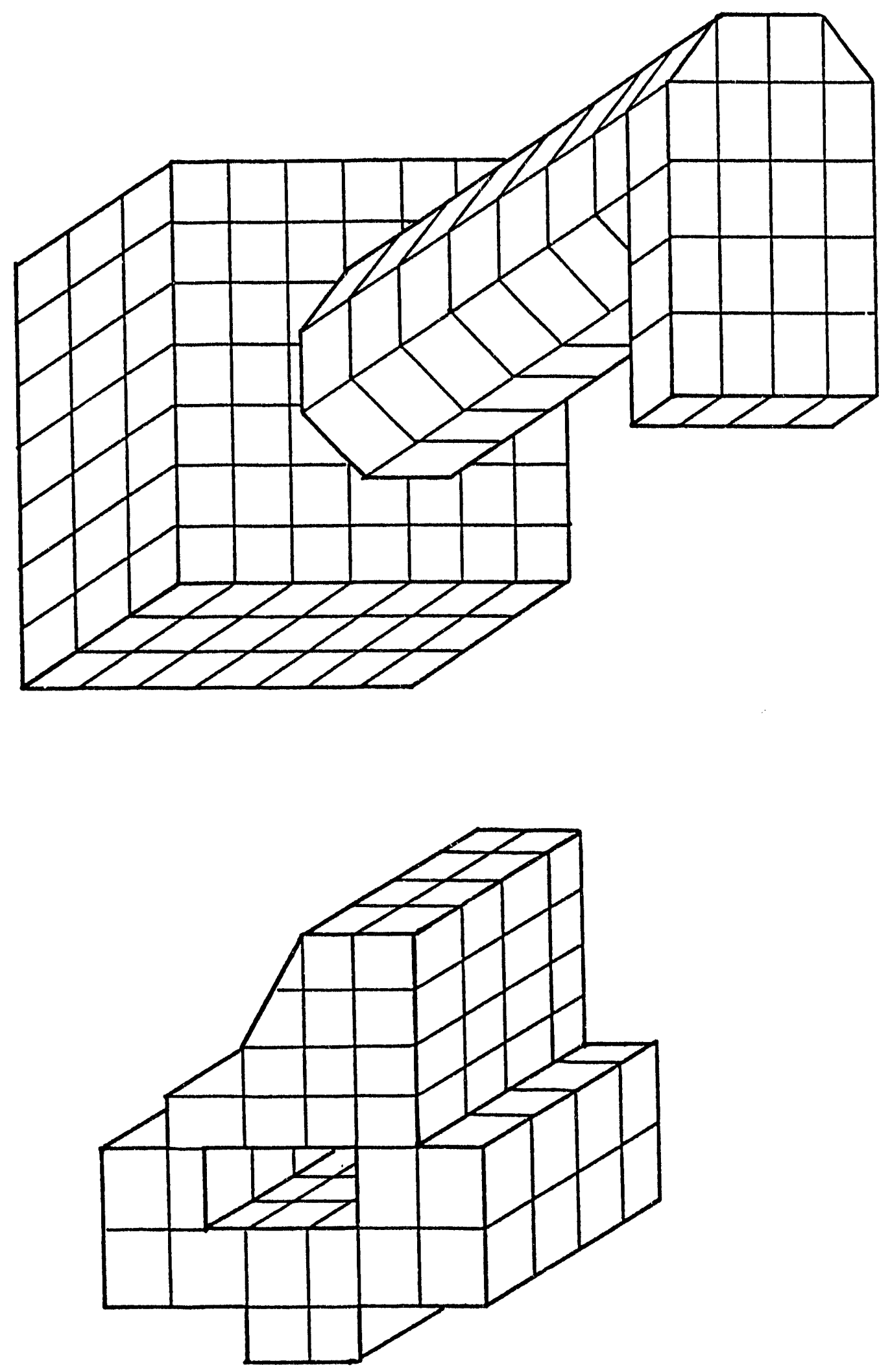

Fig. 15. Illustrations showing partitioning of sample geometries 
- Compute volume porosity $\mathrm{AL}$ and directional surface porosities $\mathrm{ALX}$, ALY, and ALZ and input this information according to the section on Cell Initialization Records in Appendix A (Input Description).

- Compute the condenser-tube numbers in each tube channel and spectfy the channel locations and flow direction according to the section on Tube Channel Location Records in Appendix A. Also specify:

NTUBES: Total number of tube channels.

NMCELL: Total number of computational cells in the tube side.

\section{Initialization}

\subsection{Introduction}

After completing the geometrical modeling, initial values of temperature, pressure, and velocity for all cells and surfaces are assigned. If one is continuing a previous run, initialization is performed through the restart capability of COMMIX.

The input of all initial and boundary values at the start of the first run can be very tedious. In COMMLX, several simplified input procedures for initialization are provided.

Lists of initialization variables for uniform and nonuniform conditions in the shell side are given in Tables 3 and 4, respectively. The variables for nonuniform initialization override those for uniform initialization. For tube-side flow, the list of initialization vartables is given in Table 5. These variables are discussed in Sec. 4.2. More details can be found in Appendix A.

\subsection{Simplified Procedures}

Shell Side

- For the initial input, specify only pressure, temperature, and velocity. The values of enthalpy and density are not required; they are calculated in the code from the equation of state and the specified pressure and temperature distributions.

- Establish initial hydrostatic pressure distributions in the entire flow domain by specifying only

-One pressure value (variable PRES $\varnothing$ ) for all $x, y$, and $z$ locations (variables are XPRESØ. YPRES $\varnothing$, and ZPRES $\varnothing)$, and

-The components of the gravity vector (varlables are GRAVX, GRAVY, and GRAVZ).

The program then calculates the entire hydrostatic-pressure field. 
Table 3. Initialization variables for uniform shell-side conditions (Input Section is DATA)

\begin{tabular}{|c|c|c|c|}
\hline Variable & $\begin{array}{l}\text { Vartable } \\
\text { Name }\end{array}$ & $\begin{array}{c}\text { Initializing } \\
\text { Region }\end{array}$ & Remarks \\
\hline \multirow[t]{2}{*}{$\begin{array}{l}\text { Temperature, } \\
{ }^{\circ} \mathrm{C}\end{array}$} & TEMPØ & $\begin{array}{l}\text { Internal } \\
\text { cell }\end{array}$ & One value for all cells \\
\hline & $\operatorname{TEMP}(\mathrm{N})$ & $\begin{array}{l}\text { Boundary } \\
\text { surface }\end{array}$ & One value for each surface \\
\hline Velocity, $\mathrm{m} / \mathrm{s}$ & VELOC(N) & $\begin{array}{l}\text { Boundary } \\
\text { surface }\end{array}$ & One value for each surface \\
\hline \multirow[t]{2}{*}{ Pressure, Pa } & PRES $\varnothing$ & $\begin{array}{l}\text { Intèrnal } \\
\text { cell }\end{array}$ & Hydrostatic pressure distribution \\
\hline & PRES(N) & $\begin{array}{l}\text { Boundary } \\
\text { surface }\end{array}$ & One value for each surface \\
\hline Heat flux, $\mathrm{W} / \mathrm{m}^{2}$ & $\operatorname{TEMP}(\mathbf{N})$ & $\begin{array}{l}\text { Boundary } \\
\text { surface }\end{array}$ & One value for each surface \\
\hline Mass flux, $\frac{\mathrm{kg}}{\mathrm{s}-\mathrm{m}^{2}}$ & VELOC(N) & $\begin{array}{l}\text { Boundary } \\
\text { surface }\end{array}$ & One value for each surface \\
\hline
\end{tabular}

- Uniform temperature distribution in the entire flow domain is obtained by specifying only one temperature value (variable TEMPØ).

- Uniform temperature and hydrostatic pressure distributions can be overridden by using cell initialization records (CIR) for the desired (1.j.k) locations.

- Uniform normal velocity and temperature for each surface are prescribed by specifying the desired values for vartables VELOC(N) and TEMP(N). respectively, where $\mathrm{N}$ is the surface number. Uniform pressure at cells adjacent to a surface is specified by PRES(N) and only needs to be specified for a surface with a uniform pressure boundary condition.

- Nonuniform velocity and temperature distributions for surface elements are specified by using the surface element initialization records (SEIR) with variables VELB and TLB. This overrides any VELOC(N) and TEMP(N) values. Nonuniform pressure distribution at cells adjacent to a surface with a specified pressure boundary condition is presented by using CIR, which override the PRES(N) specification.

- If the user wishes to initially prescribe surface heat flux instead of temperature. she/he can do so by specifying the desired value in $\mathrm{W} / \mathrm{m}^{2}$ to the 
Table 4. Initialization variables for nonuniform shell-side conditionsa

\begin{tabular}{|c|c|c|c|c|}
\hline Variable & $\begin{array}{c}\text { Variable } \\
\text { Name }\end{array}$ & $\begin{array}{l}\text { Inttializing } \\
\text { Region }\end{array}$ & $\begin{array}{l}\text { Input } \\
\text { Section }\end{array}$ & Remarks \\
\hline $\begin{array}{l}\text { Temperature. } \\
{ }^{\circ} \mathrm{C}\end{array}$ & $\begin{array}{l}\text { TL } \\
\text { TLB }\end{array}$ & $\begin{array}{l}\text { Internal cell } \\
\text { Surface element }\end{array}$ & $\begin{array}{l}\text { CIRb } \\
\text { SEIRc }\end{array}$ & $\begin{array}{l}\text { Desired internal cells } \\
\text { Desired surface elements }\end{array}$ \\
\hline Velocity, $\mathrm{m} / \mathrm{s}$ & $\begin{array}{l}\text { UL,VL,WL } \\
\text { VELB }\end{array}$ & $\begin{array}{l}\text { Internal cell } \\
\text { faces } \\
\text { Surface element }\end{array}$ & SEIR & Desired surface elements \\
\hline Pressure, $\mathrm{Pa}$ & POLD & Internal cell & CIR & Desired internal cells \\
\hline Heat flux, $w / m^{2}$ & QBN & Surface element & SEIR & Desired surface element \\
\hline Enthalpy, J/kg & $\begin{array}{l}\text { HL } \\
\text { HLB }\end{array}$ & $\begin{array}{l}\text { Internal cell } \\
\text { Surface element }\end{array}$ & $\begin{array}{l}\text { CIR } \\
\text { SEIR }\end{array}$ & $\begin{array}{l}\text { Desired internal cells } \\
\text { Desired surface elements }\end{array}$ \\
\hline Density, $\mathrm{kg} / \mathrm{m}^{3}$ & RLB & Surface & SEIR & Desired surface elements \\
\hline $\begin{array}{l}\text { Heat source. } \\
\qquad \mathrm{W} / \mathrm{m}^{3}\end{array}$ & QSOU & Internal cell & CIR & Desired internal cells \\
\hline $\begin{array}{l}\text { Turbulence } \\
\text { kinetic energy. } \\
\quad \mathrm{J} / \mathrm{kg}\end{array}$ & $\begin{array}{l}\text { TK } \\
\text { TKB }\end{array}$ & $\begin{array}{l}\text { Internal cell } \\
\text { Surface element }\end{array}$ & $\begin{array}{l}\text { CIR } \\
\text { SEIR }\end{array}$ & $\begin{array}{l}\text { Desired internal cells } \\
\text { Desired surface elements }\end{array}$ \\
\hline $\begin{array}{l}\text { Turbulence } \\
\text { dissipation rate, } \\
\text { W/kg }\end{array}$ & $\begin{array}{l}\text { TD } \\
\text { TDB }\end{array}$ & $\begin{array}{l}\text { Internal cell } \\
\text { Surface element }\end{array}$ & $\begin{array}{l}\text { CIR } \\
\text { SEIR }\end{array}$ & $\begin{array}{l}\text { Desired internal cells } \\
\text { Desired surface elements }\end{array}$ \\
\hline $\begin{array}{l}\text { Mass flow rate, } \\
\mathrm{kg} / \mathrm{s}\end{array}$ & FLOW & Surface element & SEIR & Desired surface elements \\
\hline
\end{tabular}

avarlables listed in Table 4 override those listed in Table 3.

bCIR: Cell Initialization Records.

cSEIR: Surface Element Initialization Records.

variable TEMP(N). A nonuniform heat flux distribution can be specified by using the SEIR with variable QBN. This overrides any TEMP value.

- Initial values of density and enthalpy fields are computed by the code using the equation of state and the prescribed initial temperature and pressure fields.

- Turbulence parameters ( $k$ and $\varepsilon$ ) are specified for internal cells by using the CIR with varlables TK and TD, and for surface elements by using the SEIR with variables TKB and TDB. 
Table 5. Variables for tube-side initialization

(Input Section is TUBS)

\begin{tabular}{|c|c|c|c|}
\hline Variable & $\begin{array}{l}\text { Variable } \\
\text { Name }\end{array}$ & $\begin{array}{l}\text { Initializing } \\
\text { Region }\end{array}$ & Remarks \\
\hline \multirow[t]{3}{*}{ Temperature, ${ }^{\circ} \mathrm{C}$} & TEMØTB & Internal cell & One value for all cells \\
\hline & TLBTB 1 & $\begin{array}{l}\text { Inlet boundary } \\
\text { surface }\end{array}$ & One value for each tube channel \\
\hline & TLBTB2 & $\begin{array}{l}\text { Outlet boundary } \\
\text { surface }\end{array}$ & One value for each tube channel \\
\hline \multirow[t]{3}{*}{ Velocity, m/s } & VELØTB & Internal cell & One value for all cells \\
\hline & ULBTB 1 & $\begin{array}{l}\text { Inlet boundary } \\
\text { surface }\end{array}$ & One value for one tube channel \\
\hline & ULBTB2 & $\begin{array}{l}\text { Outlet boundary } \\
\text { surface }\end{array}$ & One value for one tube channel \\
\hline Pressure, $\mathrm{Pa}$ & PREØTB & Internal cell & One value for all cells \\
\hline
\end{tabular}

- Mass flow rates corresponding to uniform mass flux are specified by VELOC(N).

- Mass flow rates for the boundary surfaces are specified using the SEIR with the variable FLOW. This overrides any value specifled through VELOC.

- The format of the CIR and SEIR is

NAME RVAL IB IE JB JE KB KE N*

An example of the CIR is

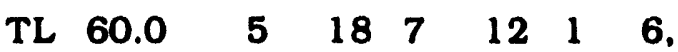

meaning that we are specifying a cell temperature of $60^{\circ} \mathrm{C}$ for all internal cells in the region having an I value from 5 to 18 . J value from 7 to 12 , and $K$ value from 1 to 6 . Thus, in this example, 504 cells are initialized by one input card.

An example of the SEIR is

$\begin{array}{lllllllll}\text { TLB } & 60.0 & 5 & 18 & 7 & 12 & 1 & 1 & 5 .\end{array}$

N for SEIR only. 
meaning that we are specifying a surface temperature of $60^{\circ} \mathrm{C}$ for all surface elements on computational cells having an I value from 5 to $18, \mathrm{~J}$ value from 7 to 12 , and $\mathrm{K}$ value from 1 to 1 for surface number 5 . We can see here that in this example one input card initializes the temperature value for $\mathbf{8 4}$ surface elements.

- Before a program reads the input data, all variables are set to zero. Consequently, only the values other than zero must be specified in the input data.

The commonly occurring values of variables are provided as default values. If the default value for a given variable is acceptable. the input for that variable need not be specified.

Tube Side

- Just as for shell stde, one need only specify temperature, pressure, and velocity for the initial input. The values of enthalpy and density are calculated in the code from the prescribed pressure and temperature distributions.

- Uniform temperature, velocity, and pressure distribution in the entire flow domain are obtained by specifying only one value for each of the variables TMPØTB, VELØTB, and PREØTB, respectively.

- The inlet and outlet velocity for each tube channel are prescribed by specifying the desired values to the variables ULBTB1(N) and ULBTB2(N). respectively, where $N$ is the tube channel number.

- The inlet and outlet temperatures for each tube channel are prescribed by specifying desired values for the variables TLBTB $1(\mathrm{~N})$ and TLBTB2(N). respectively

- The variables for tube-side initialization are listed in Table 5.

\subsection{Input Preparation}

\subsubsection{Intornal Coll Variables}

\section{Shell Side}

In the input preparation of initial cell values, the following variables must be specified:

- Three velocity components, UL, VL, and $\mathrm{WL}$ in $\mathrm{m} / \mathrm{s}$.

- Temperature $\mathrm{TL}$ in ${ }^{\circ} \mathrm{C}$.

- Pressure POLD in Pa, and

- Heat source QSOU in $\mathrm{W} / \mathrm{m}^{3}$.

As mentioned previously, only nonzero values need be specifled. Also, simplified procedures described in Sec. 4.2 should be used for specifying uniform temperature and hydrostatic-pressure distributions at the start. Use TEMP $\varnothing$ and PRES $\varnothing$ in NAMELIST/DATA/. 
hydrostatic-pressure distributions at the start. Use TEMP $\varnothing$ and PRES $\varnothing$ in NAMELIST/DATA/.

With regard to the heat source, the user has the option of either using the volumetric F sat source QSOU or specifying it through thermal structure (see Sec. 5).

Tube Side

The following variables are assumed to be uniform throughout the tubes.

- Flow velocity ULTB in $\mathrm{m} / \mathrm{s}$,

- Temperature TLTB in ${ }^{\circ} \mathrm{C}$, and

- Pressure POLDTB in Pa.

These three variables are calculated in the code by specifying VELØTB, TEM PREDTB in NAMELIST/TUBS/.

\subsubsection{Boundary Surface Variables}

Shell Side

In the initialization of boundary variables, one must specify the type of boundary conditions and the boundary values. They are discussed separately below.

- Types of Boundary Conditions

The variables used for defining the types of boundary conditions are

KFLOW: for velocity,

KTEMP: for temperature/heat flux, and

KPRES: for pressure.

The options for the velocity, temperature/heat flux, and pressure boundary conditions are listed in Tables 6-8 respectively. Detailed descriptions of these options are given in Volume I and Appendix A.

- Boundary Values

The variables VELOC(N), TEMP(N), and PRES(N) are used to initialize uniform values of velocity, temperature, and pressure. These values are specified in NAMELIST/DATA/.

If nonuniform boundary conditions are required, the input is achieved through SEIR. Table 9 lists eight variables that can be specified through SEIR. In general, we need not specify all eight variables. Of course, one can always specify uniform boundary conditions by using the SEIR.

Tube Side

- Types of Boundary Conditions

The variables used for defining the types of boundary conditions are 
Table 6. Options for shell-side velocity boundary conditions

\begin{tabular}{|c|c|c|c|}
\hline $\begin{array}{l}\text { Parameter } \\
\text { KFLOW(N) }\end{array}$ & $\begin{array}{l}\text { Boundary } \\
\text { Condition Type }\end{array}$ & $\begin{array}{l}\text { Physical } \\
\text { Boundary }\end{array}$ & Remarks $^{\mathbf{a}}$ \\
\hline-5 & $\begin{array}{l}\text { Continuative mass } \\
\text { flow outlet }\end{array}$ & Outlet & $\begin{array}{l}v_{n}^{0}=\frac{(\rho A v)^{A I}}{(\rho A)^{0}} \text { (one outlet surface } \\
\text { element) }\end{array}$ \\
\hline-4 & $\begin{array}{l}\text { Uniform velocity } \\
\text { outlet }\end{array}$ & Outlet & $\begin{array}{l}\mathbf{v}_{n}^{0}=\frac{\Sigma(\rho A v)^{A I}}{\Sigma(\rho A)^{0}} \text { (several outlet } \\
\text { surface elements) }\end{array}$ \\
\hline-3 & Free slip & $\begin{array}{l}\text { Symmetry } \\
\text { surface }\end{array}$ & $\begin{array}{l}v_{n}^{0}=0 ; \frac{\partial v_{t}^{0}}{\partial n}=0 \text { No momentum } \\
\text { diffusion }\end{array}$ \\
\hline-2 & $\begin{array}{l}\text { Continuative velocity } \\
\text { outlet }\end{array}$ & Outlet & $\begin{array}{l}v_{n}^{0}=v_{n}^{N} \text { For constant density } \\
\text { and area }\end{array}$ \\
\hline-1 & $\begin{array}{l}\text { Continuative } \\
\text { momentum outlet }\end{array}$ & Outlet & $\mathbf{v}_{n}^{0}=\frac{(\rho \mathbf{v})^{\mathbf{A}}}{\rho^{0}}$ For constant area \\
\hline 0 & No slip & $\begin{array}{l}\text { Stationary } \\
\text { solid wall }\end{array}$ & $v_{n}^{0}=0 ; v_{t}^{0}=0$ \\
\hline 1 & Constant velocity & $\begin{array}{l}\text { Inlet, } \\
\text { solid wall }\end{array}$ & $\begin{array}{l}v_{n}^{1} \neq 0, v_{t}^{1}=0 \\
v_{n}^{0}=0, v_{t}^{0}=0\end{array}$ \\
\hline 2 & $\begin{array}{l}\text { Constant mass } \\
\text { flow rate }\end{array}$ & $\begin{array}{l}\text { Inlet/ } \\
\text { outlet }\end{array}$ & $\begin{array}{l}\text { Mass flow rate specified by FLOW } \\
\text { in the SEIR }\end{array}$ \\
\hline $100+N F$ & $\begin{array}{l}\text { Uniform transient } \\
\text { velocity }\end{array}$ & Inlet & $\begin{array}{l}v_{n}^{1}(t)=\operatorname{VELOC}(N) f(t) \\
f(t)=\text { Dimensionless function } \\
\text { number }\end{array}$ \\
\hline $200+N F$ & $\begin{array}{l}\text { Uniform transient } \\
\text { mass flow rate }\end{array}$ & Inlet & FLOW $(t)=\operatorname{VELOC}(\mathrm{N}) \mathrm{f}(\mathrm{t})$ \\
\hline
\end{tabular}

aSuperscripts: $0=$ Outlet or boundary value; $A \mathrm{I}=$ adjacent internal cell; $1=$ inlet value; $\mathrm{n}=$ normal to boundary surface; $\mathrm{t}=$ tangential to boundary surface. 
Table 7. Options for shell-side temperature/heat flux boundary conditions

\begin{tabular}{|c|c|c|c|}
\hline $\begin{array}{l}\text { Parameter } \\
\text { KTEMP(N) }\end{array}$ & $\begin{array}{l}\text { Boundary } \\
\text { Condition Type }\end{array}$ & $\begin{array}{l}\text { Physical } \\
\text { Boundary }\end{array}$ & Remarks \\
\hline 1 & $\begin{array}{l}\text { Constant } \\
\text { temperature }\end{array}$ & $\begin{array}{l}\text { Inlet, } \\
\text { solid wall }\end{array}$ & $\begin{array}{l}T_{w}=\text { constant, } \\
q_{w} \text { is calculated }\end{array}$ \\
\hline $100+N F$ & $\begin{array}{l}\text { Transient } \\
\text { temperature }\end{array}$ & $\begin{array}{l}\text { Inlet, } \\
\text { solid wall }\end{array}$ & $\begin{array}{l}T_{w}(t)=T_{w}(0) f(t) \\
q_{w} \text { is calculated }\end{array}$ \\
\hline 200 & $\begin{array}{l}\text { Constant heat } \\
\text { fux }\end{array}$ & Solid wall & $\begin{array}{l}\mathbf{q}_{\mathbf{w}}=\text { constant } \\
\mathbf{T}_{\mathbf{w}} \text { is calculated }\end{array}$ \\
\hline $300+N F$ & $\begin{array}{l}\text { Transient heat } \\
\text { flux }\end{array}$ & Solid wall & $\begin{array}{l}q_{w}(t)=q_{w}(0) f(t) \\
T_{w} \text { is calculated }\end{array}$ \\
\hline 400 & Adiabatic & $\begin{array}{l}\text { Outlet, } \\
\text { symmetry }\end{array}$ & $q_{w}=0.0$ \\
\hline
\end{tabular}

Table 8. Options for shell-side pressure boundary conditions

\begin{tabular}{|c|c|c|c|}
\hline $\begin{array}{l}\text { Parameter } \\
\text { KPRES(N) }\end{array}$ & $\begin{array}{l}\text { Boundary } \\
\text { Condition }\end{array}$ & $\begin{array}{l}\text { Physical } \\
\text { Boundary }\end{array}$ & Remarks \\
\hline 0 & - & - & $\begin{array}{l}\text { No pressure bounda } \\
\text { condition is applied }\end{array}$ \\
\hline 1 & Constant pressure & Inlet-outlet & $P_{w}=$ constant \\
\hline $100+\mathrm{NFa}$ & Transtent pressure & Inlet-outlet & $P_{w}(t)=P_{w}(0) F(N F)$ \\
\hline \multicolumn{4}{|c|}{ aNF is the transient function number; $F(N F)$ is the NFth transient function. } \\
\hline \multicolumn{4}{|c|}{ Table 9. Shell-side variables that can be specified through SEIR } \\
\hline \multicolumn{2}{|r|}{ Variable } & \multicolumn{2}{|c|}{ FORTRAN Input } \\
\hline \multicolumn{2}{|c|}{ Enthalpy } & HIB & $\mathrm{J} / \mathbf{k g}$ \\
\hline \multicolumn{2}{|c|}{ Mass flow rate } & FLOW & $\mathrm{kg} / \mathrm{s}$ \\
\hline \multicolumn{2}{|c|}{ Heat flux } & QBN & $\mathrm{w} / \mathrm{m}^{2}$ \\
\hline \multicolumn{2}{|c|}{ Density } & RLB & $\mathrm{kg} / \mathrm{m}^{3}$ \\
\hline \multicolumn{2}{|c|}{ Temperature } & TLB & ${ }^{\circ} \mathrm{C}$ \\
\hline \multicolumn{2}{|c|}{ Normal velocity } & VELB & $\mathrm{m} / \mathrm{s}$ \\
\hline \multirow{2}{*}{\multicolumn{2}{|c|}{$\begin{array}{l}\text { Turbulence kinetic energy } \\
\text { Turbulence dissipation rate }\end{array}$}} & TKB & $\mathrm{J} / \mathrm{kg}$ \\
\hline & & TDB & $\mathrm{W} / \mathrm{kg}$ \\
\hline
\end{tabular}


where $\mathrm{I}=1$ for the inlet boundary and $\mathrm{I}=2$ for the outlet boundary.

- Boundary Values

For flow inside the condenser tubes, the boundary values of velocity and temperature are specified in NAMELIST/TUBS/ by using the following variables:

ULBTB1(N): ' for inlet velocity. ULBTB2(N): for outlet velocity, TLBTB 1(N): for inlet temperature. TLBTB2(N): for outlet temperature, where $\mathrm{N}$ is the tube channel number.

\section{Thermal-Structure Modeling}

\subsection{Introduction}

The purpose of incorporating a thermal-structure model in COMMLX-PPC is to facilitate consideration of heat-transfer interaction between fluid and structures. The model implemented in COMMLX solves one-dimensional heat conduction equations for all solid structures, including condenser tubes. It calculates temperature distribution in solids and heat transfer from solids to surrounding fluids. In general, the output from the thermal-structure model is a heat source/sink term for the fluid energy equation. However, because the energy equation is not used for condenser modeling, the heat source/sink term is used to calculate the steam condensation rate.

The basic equations, formulations, and features of the thermal-structure model of condenser tubes and other internal solid structures are described in Vol. l. Here, we describe only user-related aspects of the model in a general way.

\subsection{Some Concepts and Definitions}

A thermal structure is a solid structure, in a fluid-flow domain, that thermally interacts with the surrounding fluid. It can be planar, cylindrical, or spherical. The COMMIX-PPC model permits only structures with axes aligned with one of the three coordinate axes, as illustrated in Fig. 16. In a given flow domain, more than one thermal structure can be modeled if desired.

A thermal structure is identified by its geometric and physical features, i.e.,

- All solid structures having the same grid axis alignment, same geometric features, and same physical characteristics can be collectively modeled as one thermal structure, although they may be spread out physically in the fluid domain. Examples are fuel pins in a nuclear reactor, cooling tubes in a power plant condenser. 


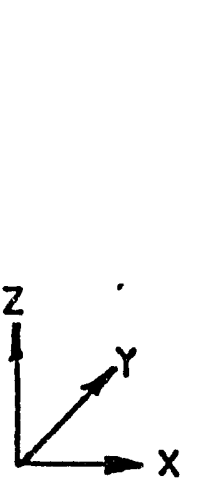

$x$

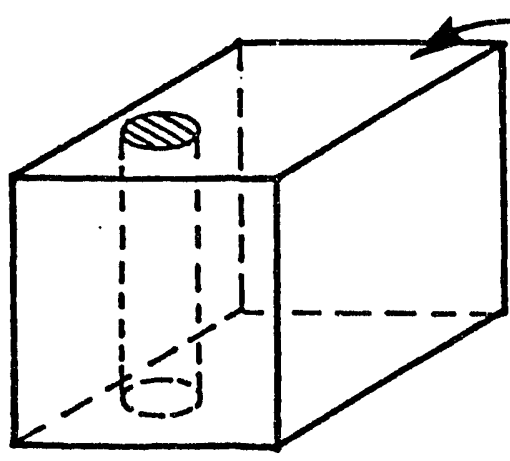

(a)

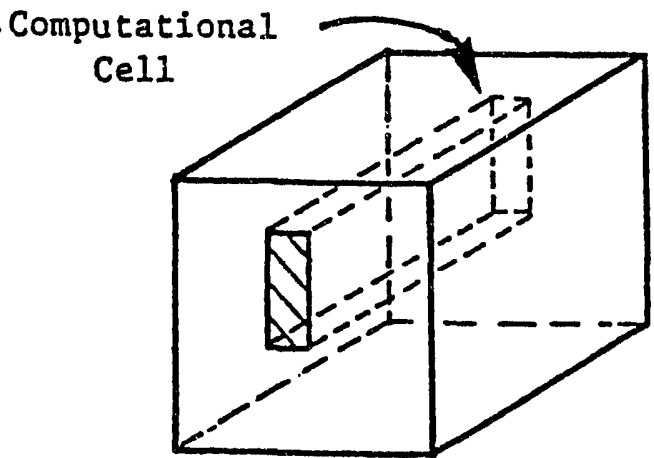

(b)

Fig. 16. Illustrations showing thermal-structure alignment:

(a) cylindrical structure aligned to 2 axis;

(b) planar structure aligned to $y$ axis

- A solid structure with a single geometric feature but heterogeneous physical characteristics cannot be modeled as one thermal structure. An example is fuel pins with different material composition in different axial sections.

In COMMIX-PPC computations, each thermal structure is partitioned by grid planes normal to the structure axis to form several thermal structure elements, as shown in Fig. 17. Each element has its own internal temperature distribution because the heat conduction equation is solved for each element.

A thermal structure has two surfaces, outer and inner. These surfaces can be adiabatic or they can interact with fluid, as shown in Fig. 18. Each structural-surface element is permitted to interact with only one fluid cell. However, a fluid cell can interact with more than one structure surface. This can be seen in Figs. 19 and 20.

Each structure may be composed of several material segments. Figure 21 illustrates the cross section of a structure element having

- Outside and inside surfaces, numbered 1 and 2, respectively,

- Three different materials separated by gaps, and

- Each material region divided into several partitions.

\subsection{Thermal-Structure Subroutines}

Three major subroutines are related to the thermal-structure model:

- HSTRUC: Computes heat transfer coefficients for the outer and inner surfaces of all thermal-structure elements. It uses local velocities 

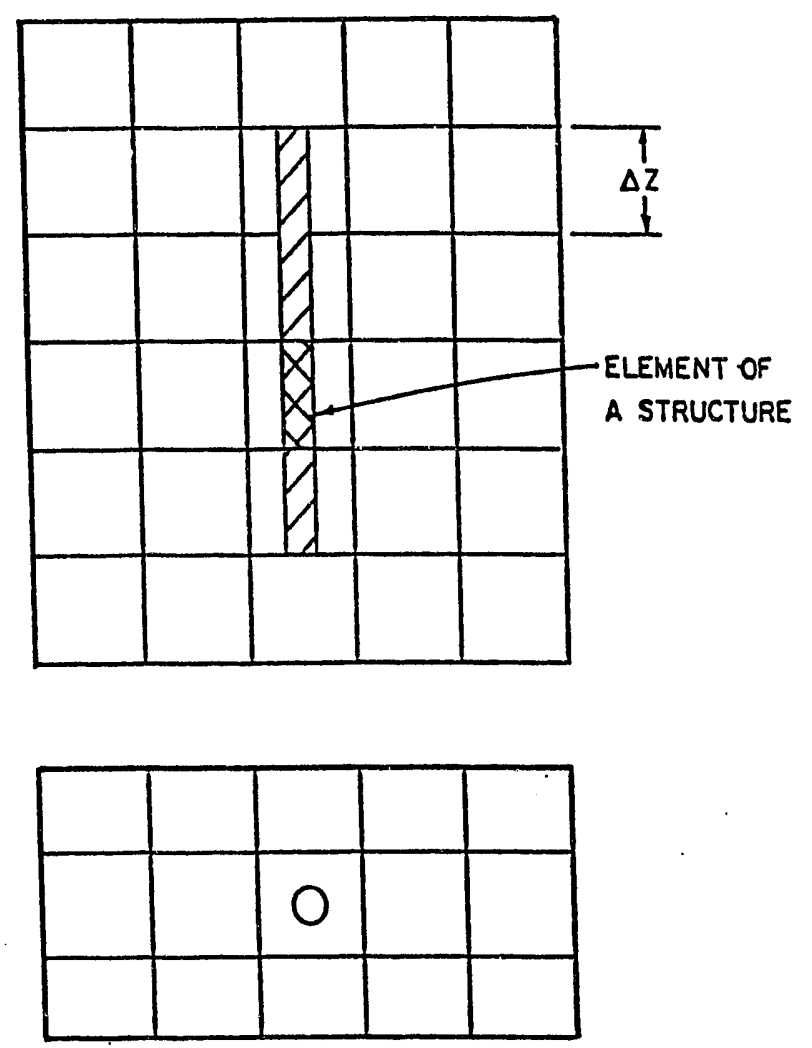

Fig. 17. An element of a thermal structure

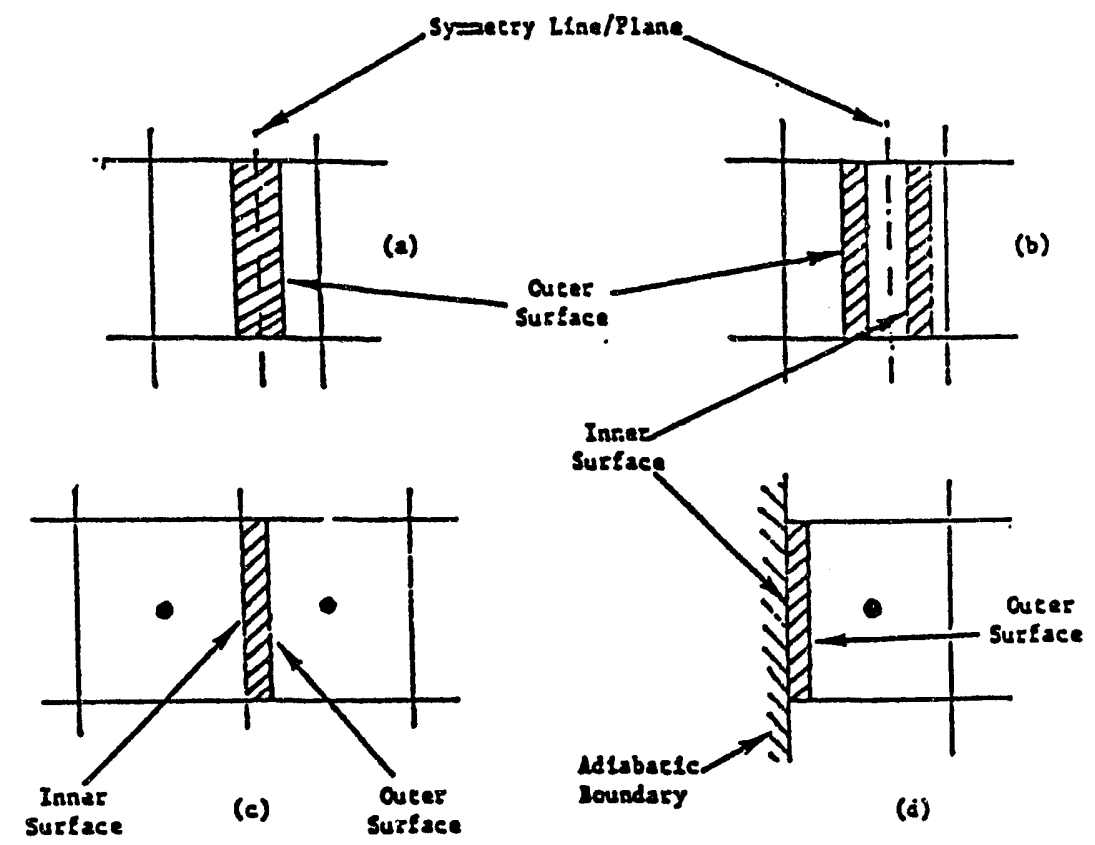

Fig. 18. Element of a thermal structure, showing outer and inner surfaces 


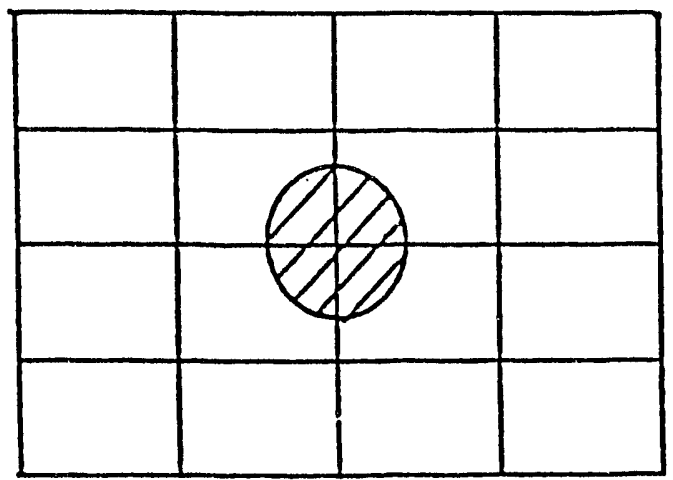

Fig. 19. Four quarter-cylindrical structures, each interacting with one fluid cell

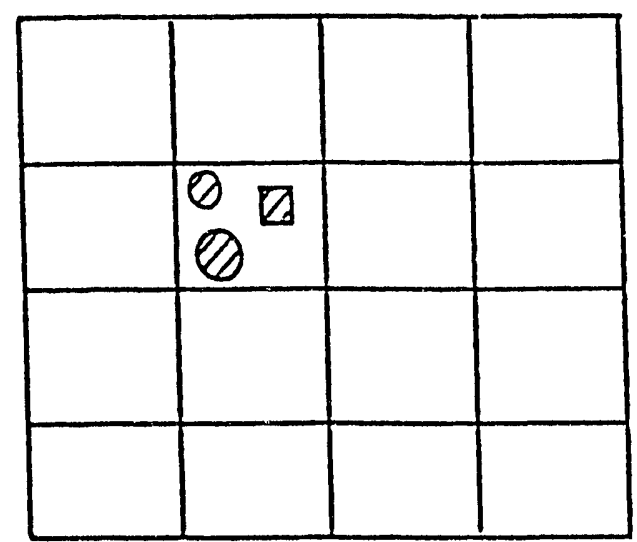

Fig. 20. Multiple structures interacting with a single fluid cell

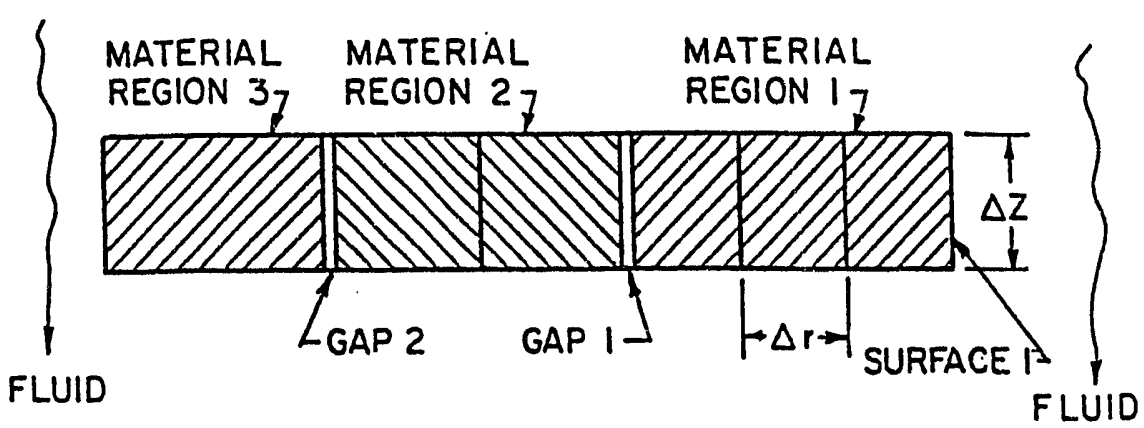

CROSS SECTION A-A

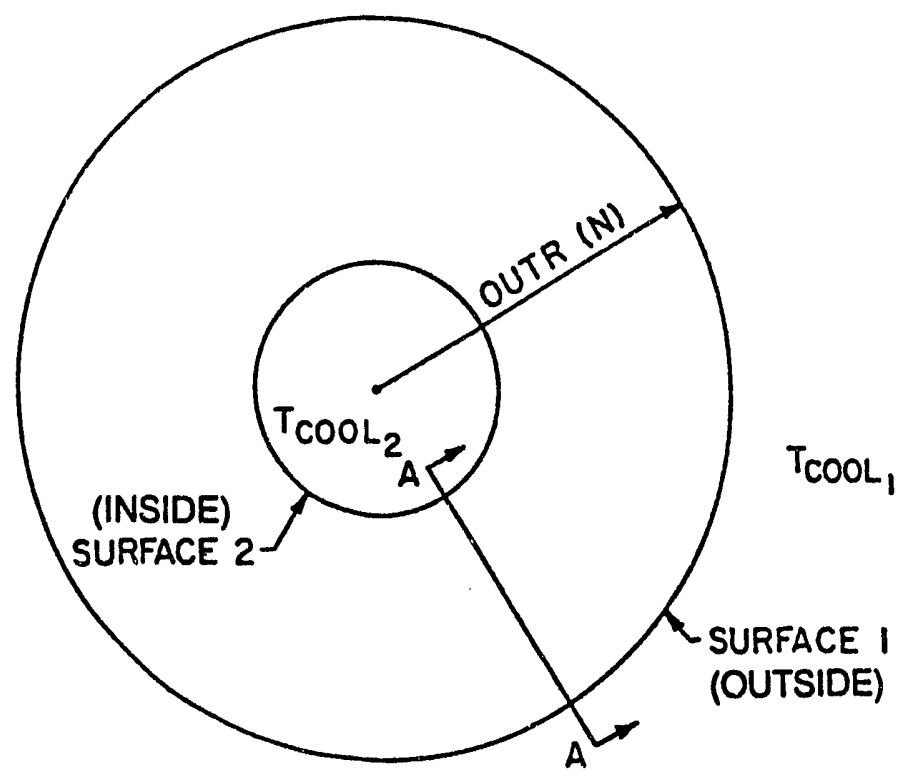

Fig. 21. Typical structure element showing material regions and gaps 
and thermal properties to calculate Reynolds number and Prandtl number and appropriate user-specified heat transfer correlations to evaluate the heat transfer coefficient.

- QSTRUC: Solves the heat conduction equations for each element and computes the heat source term for the fluid energy equation.

- TSTRUC: Solves the heat conduction equations for each element and computes the temperature distribution of the thermal-structure element.

The other three subroutines for this model are TSCAN, INPSTR, and PSTRUC. The functions of TSCAN are to scan input and determine storage requirements; INPSTR reads thermal-structure input and assigns appropriate markers and pointers; and PSTRUC prints results related to thermal structures.

\subsection{Modeling Recommendations}

The following is a list of recommendations relating to thermal-structure modeling

- For steady-state analysis, one needs to model only those solid structures with heat sources.

- For transient analysis, all solid structures, with or without heat source, must be modeled.

- Only plane, cylindrical, or spherical shapes are permitted in COMMIX-PPC. If a structure does not conform to one of these shapes, the user should make some approximations.

- COMMIX-PPC permits modeling of any multiple or fraction of a structure as one thermal structure; e.g., if there are 10 condenser tubes passing through one computational cell, as shown in Fig. 22a, the group of condenser tubes is modeled as one thermal structure with surface area equal to 10 times the surface area of a single tube. On the other hand, the user may wish to model only a fraction of a tube as one thermal structure, as illustrated in Fig. 22b.

- A slab structure (Fig. 23) can be considered as either a

-Two-sided thermal structure with surface area for each side $=\Delta y \Delta z$ and thickness $=t$, or

-One-sided thermal structure of thickness $t / 2$, with one adiabatic inner surface, with outer surface area of $2 \Delta y \Delta z$.

- In many cases, we find, for computational purposes, that the solid structures can be considered uniformly distributed, e.g.. fuel pins in a reactor core. cooling tubes in power condensers. An alternative means has been provided for specifying the heat transfer surface area to model such uniformly distributed thermal structures. This is done through the variable RODFR. which has different meanings depending on what and how we specify. This can best be explained through examples. 


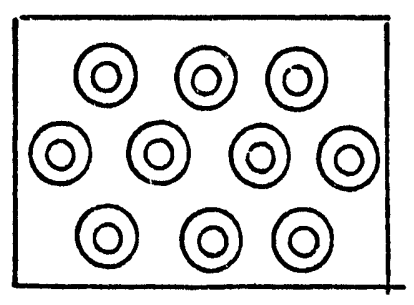

(a)

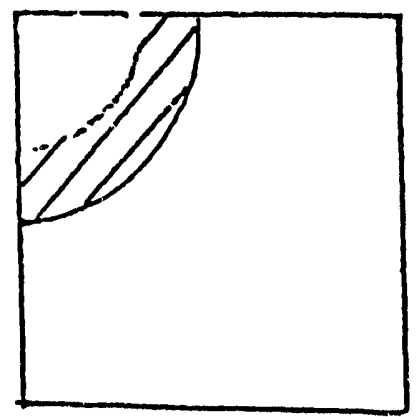

(b)

Fig. 22. Modeling (a) multiple structures or (b) fraction of a structure as one thermal structure

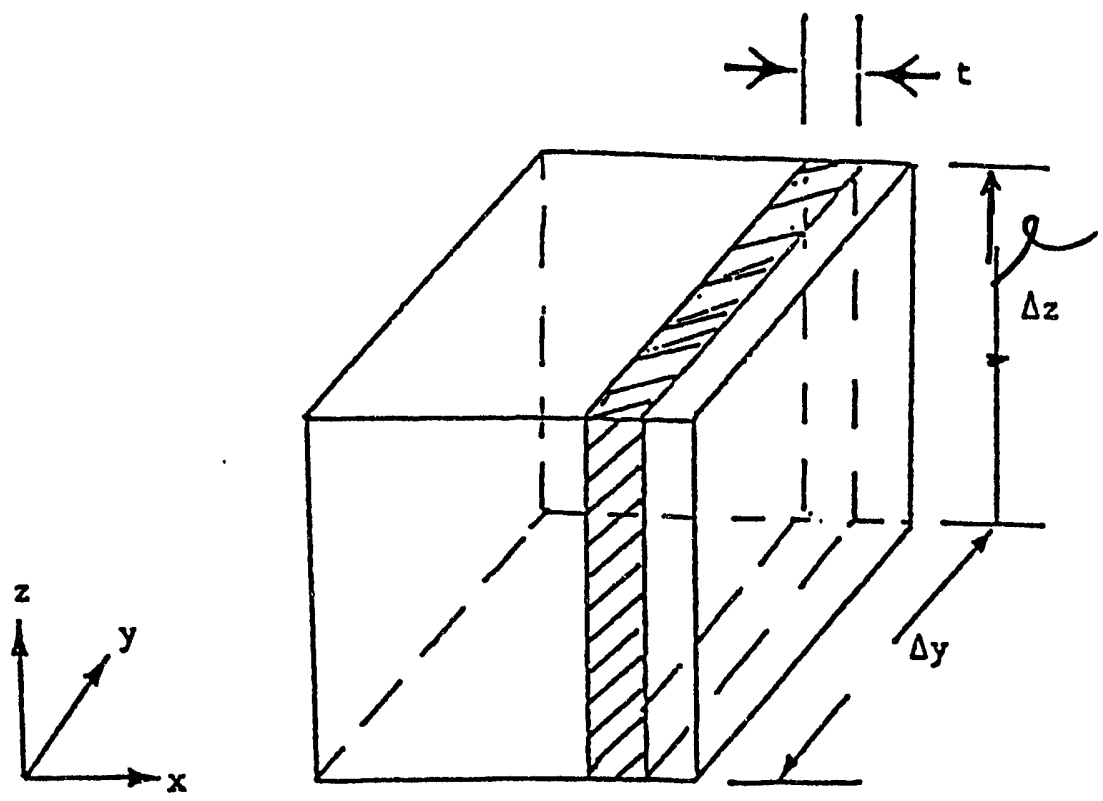

Fig. 23. Slab structure element

Positive RODFR: COMMIX -PPC computes heat transfer surface area using the following equations:

\section{Cylindrical Structure}

Surface Area $=\left(2 \pi r \Delta Z_{1}\right)$ RODFR

\section{Plane Structure}

Surface Area $=$ RODFR

\section{Spherical Structure}

Surface Area $=4 \pi r^{2}$ RODFR.

Here, $r$ is the surface radius (either outer surface or inner surface) and $\Delta Z_{1}$ is the axial length of the coolant cell. Therefore, the definition of RODFR is 
RODFR = Number or fraction of rods interacting with the fluld cell, for a cylindrical or spherical structure, and

RODFR = Surface area, for a slab-type structure. Hence, for Fig. 22a. RODFR $=10$, and for Fig. 22b. RODFR = 0.5.

Negative RODFR: COMMIX-PPC uses the following equations to compute the heat transfer surface area:

\section{Cylindrical Structure}

Surface Area $=\left(2 \pi r \Delta Z_{i} \mid R O D F R I\right) * \begin{gathered}\text { (Cell area normal to the } \\ \text { structure axis }) .\end{gathered}$

\section{Plane Structure}

Surface Area $=\mid$ RODFR $\mid$ (Cell area parallel to the structure surface
area $).$

\section{Spherical Structure}

Surface Area $=4 \pi r^{2} \mid$ RODFR $\mid *$ Cell Volume

Therefore, negative RODFR means

$$
\mid \text { RODFR } \mid=\frac{\text { number (or fraction of rods) }}{\text { cell cross- sectional area }} \text { for cylindrical structures, }
$$

or

$$
|R O D F R|=\frac{\text { slab heat transfer surface area }}{\text { cell cross }- \text { sectional area }} \text { for slab-type structures, }
$$

or

$$
|R O D F R|=\frac{\text { number of spheres }}{\text { cell volume }} \text { for spherical structures. }
$$

Hence,

RODFR $=-2 / \mathrm{ab}$

for the cell at the upper left corner in Fig. 24.

$$
\begin{aligned}
\text { RODFR } & =-\frac{1}{\pi\left(r_{2}^{2}-r_{1}^{2}\right)} \\
& =-\frac{1}{\pi\left(r_{1}+r_{2}\right)\left(r_{2}-r_{1}\right)}
\end{aligned}
$$

for the illustration in Fig. 25, and

$$
\text { RODFR }=-1
$$

for the illustration in Fig. 23. 


2b $b$\begin{tabular}{cc|ccc|}
\multicolumn{1}{c}{$a$} & \multicolumn{3}{c|}{1.50} \\
\hline 0 & 0 & 0 & 0 & 0 \\
\hline 0 & 0 & 0 & 0 & 0 \\
0 & 0 & 0 & 0 & 0 \\
\hline 0 & 0 & 0 & 0 & 0 \\
0 & 0 & 0 & 0 & 0 \\
0 & 0 & 0 & 0 & 0 \\
\hline
\end{tabular}

Fig. 24. Uniformly distributed rod bundles in a nonuniform grid

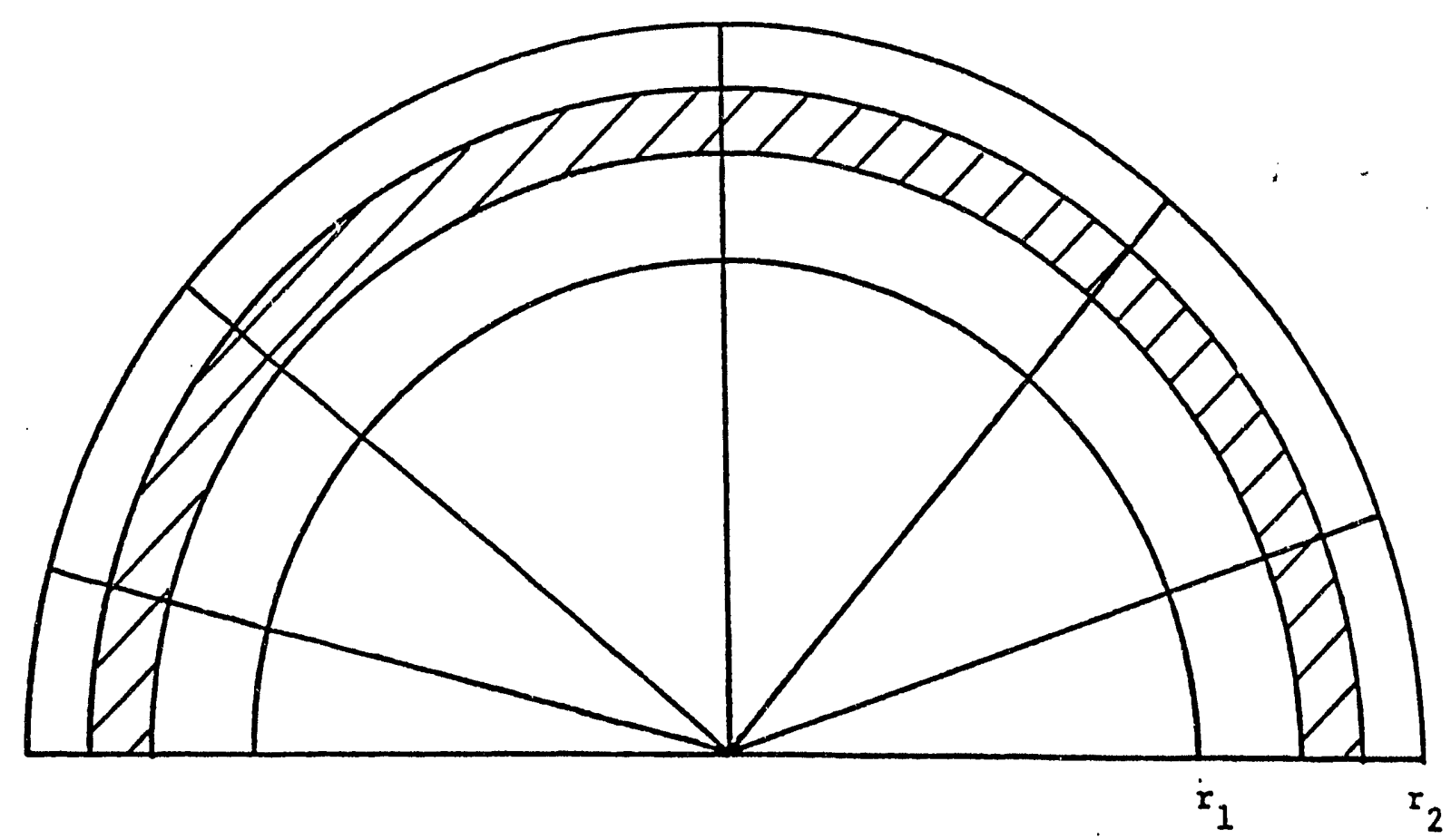

Fig. 25. Cylindrical shell with nonuniform azimuthal grid

\subsection{Input Preparation}

\subsubsection{Introduction}

There are four sections of COMMLX-PPC input where the specified information is ditectly related to the thermal structure. They are:

- NAMELIST/GEOM/.

- NAMELIST/DATA/. 
- Thermal-Structure Prototype Records.

- Thermal-Structure Location Records.

The first two are described in Sec. 5.5.2, the third, in Sec. 5.5.3, and the fourth, in Sec. 5.5.4. In addition. the inputs must also include material properties and heat transfer correlations (described in Sec. 8).

\subsubsection{NAMELIST-Input}

- NAMELIST/GEOM/: Here, we input the variable ISTRUC=1, which commands that the thermal-structure-related input is to be read and computation is to be performed.

- NAMELIST/DATA/: Here, the TS-related variable is NEWTS, which we specify NEWTS $=1$ only if we want to

-Modify or update information related to thermal structure using the restart option, or

-Input new information related to thermal structure

at the start of either a steady-state run (ISTATE $=0$ ) or a transient run (ISTATE $=2$ ). This varlable comes into operation only with ISTRUC $=1$ and ISTATE $=0$ or 2.

\subsubsection{Prototype Records}

The thermal-structure prototype records are records where we input all geometric and physical information for all thermal structures. A detailed description of this input is given in Appendix A. Some input-related rules are briefly described below.

- For each TS there is a set of input records.

- Each set contains several NAMELIST records. They are to be in the following order:

$\& T$ Information about thermal structure,

\&F Information about outer surface fluid,

\&M Information about Material 1 and Gap 1

\& $M$ Information about Material 2 and Gap 2

$\bullet$

-(Include one NAMELIST/M/ for each material region in a thermal structure.)

\&F Information about inner-surface fluid. This record is included only if the TS is two-sided. If the inner surface is adiabatic or a symmetry boundary, this record is not required. 
- The numbering of material regions begins as we traverse from outer surface to inner surface. To lllustrate the ordering system, three examples are presented.

Example 1: Ordering sequence of records for TS shown in Fig. 26a:

\& $T \quad N=, I X Y Z=, N T=, R O D F R=, O U T R=, \& E N D$

$\& \mathrm{~F} \quad$ IHT $=$, \&END

\& $M \quad M I=N P=, D R=, Q=, \& E N D$

\& $M \quad M I=N P=, D R=, Q=, S G A P=, H G A P=, \& E N D$

\&F IHT $=$, \&END

Example 2: Ordering sequence of records for TS shown in Fig. 26b:

\& $T \quad N=, L X Y Z=, N T=, R O D F R=, O U T R=, \& E N D$

\&F IHT $=, \& E N D$

$\& M \quad M I=, N P=, D R=, Q=, S G A P=, H G A P=, \& E N D$

$\& M \quad M I=, N P=, D R=, Q=, S G A P=, H G A P=, \& E N D$

$\& \mathrm{~F} \quad \mathrm{IHT}=, \quad \& \mathrm{END}$

Example 3: Ordering sequence of records for TS shown in Fig. 26c:

\& $T \quad N=, L X Y Z=, N T=, R O D F R=, O U T R=, \& E N D$

\&F IHT $=$, \&END

$\& M \quad M I=, N P=, D R=, Q=, S G A P=, H G A P=, \& E N D$

$\& M \quad M I=, N P=, D R=, Q=, S G A P=, H G A P=, \& E N D$

$\& M \quad M I=, N P=, D R=, Q=, S G A P=, H G A P=, \& E N D$

$\& M \quad M I=, N P=, D R=, Q=, S G A P=, H G A P=, \& E N D$

The meanings of all FORTRAN variables referred to in the TS prototype records are given in Table 10.

If a fluid or an adiabatic surface follows a material, the gap properties are ignored. The gap properties are used for a given material only when another material follows.

If there is no gap between two materials, we specify a high heat transfer coefficient, e.g.,

SGAP $=0.0, \quad H G A P=1 . E 30$. 


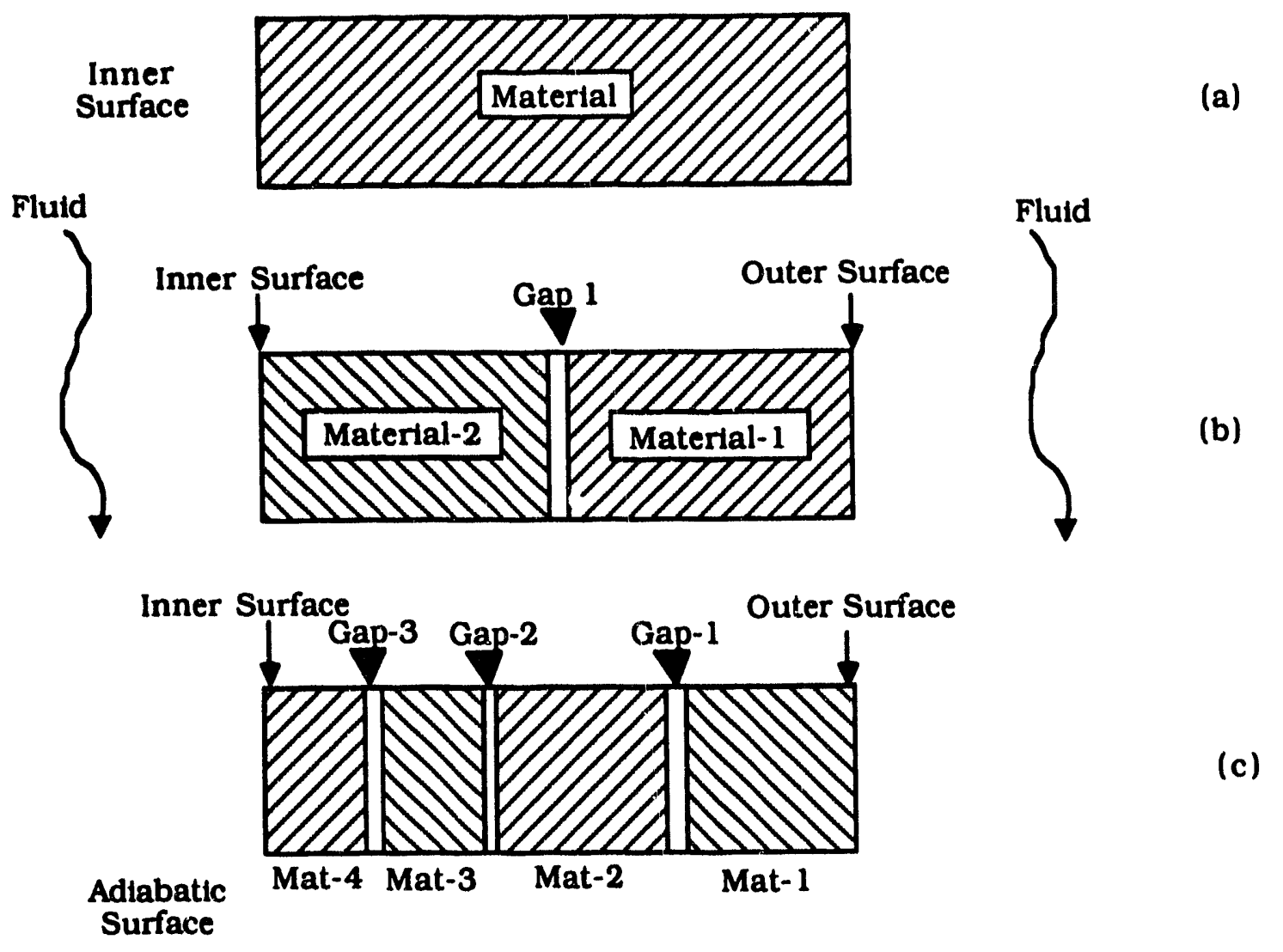

Fig. 26. Numbering system of thermal-structure material regions

Table 10. FORTRAN variables for TS prototype records

\begin{tabular}{cl}
\hline Variable & \\
\hline N & TS\# \\
LXYZ & Number to describe shape and axis alignment (see Appendix A for numbers) \\
NT & Transient function number for heat sources \\
RODFR & Rod fraction to specify surface area \\
OUTR & Outer-surface radius $(\mathrm{m})$ \\
IHT & Heat-transfer correlation number \\
MI & Material number (see Sec. 8$)$ \\
NP & Number of grid partitions $\mathrm{in}$ the material for finite-difference analysis \\
DR & Thickness of each partition $(\mathrm{m})$ \\
8 & Volumetric heat source $\left(\mathrm{W} / \mathrm{m}^{3}\right)$ \\
SGAP & Gap thickness $(\mathrm{m})$ \\
HGAP & Gap heat transfer coefficient $\left(\mathrm{W} / \mathrm{m}^{2 \circ} \mathrm{C}\right)$ \\
\hline
\end{tabular}




\subsubsection{Location Records}

The purpose of the location records is to input TS-element location information. The format of specifying the lucation information is similar to that of boundary value and internal-cell initialization records. The FORMAT is $(A 4,714)$ :

or

$$
\begin{array}{llllllll}
\text { OUT } & \mathrm{N} & \text { IB } & \text { IE } & \text { JB } & \text { JE } & \text { KB } & \mathrm{KE}
\end{array}
$$

$$
\text { IN N IB IE JB JE } \quad \text { KB } \quad \text { KE }
$$

For a two-sided structure, we specify OUT to describe the locations of the outer-surface cells and IN to describe the locations of the inner-surface cells. For one-sided structures, we have either "IN" or "OUT" records only. The variable $N$ stands for the TS number and IB ... KE stands for the beginning and ending of $1, j$, and $k$ indices. For thermal-structure modeling of condensers, the locations of the outer- and inner-surface cells of the tubes are the same.

To illustrate the location input records, we consider the simple geometry shown in Fig. 27. The inputs for the thermal structures are as follows:

$\begin{array}{llllllll}\text { OUT } & 1 & 2 & 2 & 1 & 4 & 4 & 9 \\ \text { OUT } & 2 & 8 & 8 & 1 & 5 & 9 & 9 \\ \text { OUT } & 2 & 6 & 6 & 1 & 5 & 9 & 9 \\ \text { OUT } & 3 & 8 & 8 & 1 & 5 & 2 & 2 \\ \text { IN } & 3 & 8 & 8 & 1 & 5 & 1 & 1\end{array}$

\section{Force-Structure Modeling}

\subsection{Introduction}

The purpose of force-structure modeling in COMMIX-PPC is to facilitate consideration of flow resistance due to irregular geometry and the presence of solid structures in a flow domain. In COMMIX-PPC, such resistances are accounted for by introducing an additional distributed-resistance source term in the momentum equation. To incorporate this distributed-resistance source term in the computation, certain information is needed.

\subsection{Flow Resistance Correlation}

In the literature, many different equations have been used to express pressure drop due to obstructions, e.g..

$$
\begin{aligned}
\Delta p & =4 \frac{L}{D} \frac{1}{2} \rho v^{2} f, \\
& =\frac{L}{D} \frac{1}{2} \rho v^{2} C_{D} . \\
& =\frac{1}{2} \rho v^{2} K .
\end{aligned}
$$




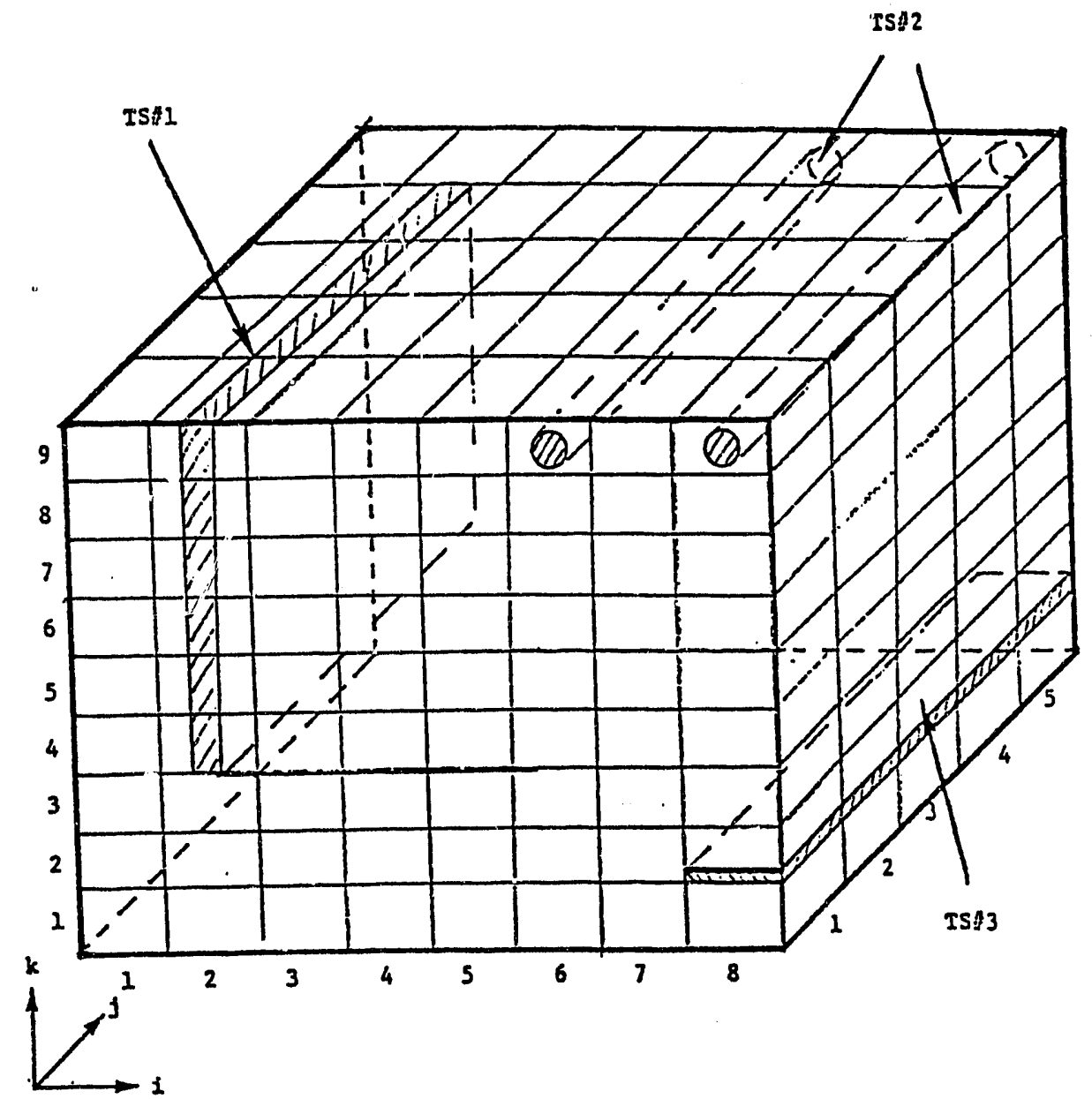

Fig. 27. TS location input

The coefficients $f, C_{D}, K$, etc. have been given different names, e.g., Fanning friction factor, Darcy friction factor, drag coefficient, loss coefficient, etc., depending on the equation.

In COMMLX-PPC, the following general forms are used:

$$
\Delta \mathrm{p}=\mathrm{C}_{1} \frac{\mathrm{L}}{\mathrm{D}} \rho \mathrm{v}^{2} \mathrm{f} \text {. }
$$

which, in terms of distributed resistance $R$, is

$$
R=\frac{\Delta p}{L}=C_{1} \rho \frac{v|v|}{D} f .
$$

Here, $L[\Delta x(\Delta r), \Delta y(r \Delta \theta)$, or $\Delta z]$ is the cell length of a momentum control volume. $D$ is the hydraulic diameter or characteristic length, and $C_{1}$ is the coefficient that accounts for different definitions of friction factor $f$.

Friction factor $f$ in Eqs. 6.2 and 6.3 is generally a function of the Reynolds number. In COMMIX-PPC, the following forms of the friction factor correlation are used:

$$
f=a_{\ell} \operatorname{Re}^{b_{\ell}}+c_{\ell}
$$


for laminar flow when the Reynolds number Re $(=\rho u d / \mu)$ is less than a predefined transition Reynolds number $R_{t r}$, and

$$
f=a_{t} \operatorname{Re}^{b_{t}}+c_{t}
$$

for turbulent flow (Re $>$ Retr). The subscripts $\iota, t$, and tr stand for laminar, turbulent, and transition, respectively.

\subsection{Input Requirements}

With reference to the form of the equations in the preceding section, the following inputs are required.

Shell Side

- Variables $C_{1}$ (FORCEF) and D(CLENTH) for the computation of the resistance-source term (Eqs. 6.2 and 6.3),

- Variables a $(A C O R R L), \ldots . ., c_{t}(C C O R R T)$, and Retr (REYTRN) for the computation of the friction factor $f(E q .6 .4)$, and

- Reference length d (REYLEN) in Reynolds number Re.

Additional input requirements are

- NEWFOR A signal to inform that new force-structure input must be read.

- NFORCE Number of force structures.

- NCORR Number of friction factor correlations.

- ICORR Linkage between a force structure and a correlation number, e.g., $\operatorname{ICORR}(3)=4$ means that correlation 4 is to be used for Force Structure 3.

- Force-structure locations.

All input variables related to force structure are listed in Table 11.

Tube Side

- Tube diameter for computing Reynold number Re,

- Variables ae (ATBL), ....., $C_{t}$ (CTBT), and $R e_{t r}(R E T R N)$ for computing friction factor $f$ (Eq. 6.4), and

- Varlables $C_{1}$ (FORCTB) for computing the resistance term (Eq. 6.3).

All input variables related to the tube-side force structure are listed in Table 12.

\subsection{Force-Structure Locations}

Information on force-structure location is provided through force-structure specification records. The FORMAT is (A4, 714): 
Table 11. Input variables related to shell-side force-structure modeling

\begin{tabular}{|c|c|c|c|}
\hline $\begin{array}{l}\text { FORTRAN } \\
\text { Namea }^{a}\end{array}$ & $\begin{array}{l}\text { Description of } \\
\text { Variable }\end{array}$ & $\begin{array}{l}\text { COMMIX Input } \\
\text { Section }\end{array}$ & Remarks \\
\hline NFORCE & $\begin{array}{l}\text { No. of force } \\
\text { structures }\end{array}$ & NAMELIST/GEOM/ & -- \\
\hline NEWFOR & $\begin{array}{l}\text { Signal for new } \\
\text { force-structure } \\
\text { input }\end{array}$ & NAMELIST/DATA/ & $\begin{array}{l}=0 \text { for no, }=1 \text { for yes; } \\
\text { applicable for ISTATE }=0 \\
\text { or ISTATE }=2\end{array}$ \\
\hline CLENTH(NF) & $\begin{array}{l}\text { D (Eqs. } 6.2 \\
\text { and } 6.3 \text { ) }\end{array}$ & NAMELIST/DATA/ & $\begin{array}{l}\text { Given a negative value if } \\
\text { desire to use Eq. } 6.1 c \text {; 1.e., } \\
\text { when } L=D\end{array}$ \\
\hline FORCEF(NF) & $\begin{array}{l}C_{1} \text { (Eqs. } 6.2 \\
\text { and 6.3) }\end{array}$ & NAMELIST/DATA/ & -- \\
\hline NCORR & $\begin{array}{l}\text { No. of } \\
\text { correlations }\end{array}$ & NAMELIST/DATA/ & -- \\
\hline ICORR(NF) & $\begin{array}{l}\text { Correlation } \\
\text { linkage }\end{array}$ & NAMELIST/DATA/ & See Sec. 6.3 \\
\hline ACORRL(N) & al (Eq. 6.4a) & NAMELIST/DATA/ & -- \\
\hline BCORRL(NC) & $\mathrm{b}_{\ell}$ (Eq. 6.4a) & NAMELIST/DATA/ & -- \\
\hline CCORRL(NC) & $c_{\ell}$ (Eq. 6.4a) & NAMELIST/DATA/ & -- \\
\hline ACORRT(NC) & $a_{t}$ (Eq. 6.4b) & NAMELIST/DATA/ & -- \\
\hline BCORRT(NC) & $b_{t}$ (Eq. 6.4b) & NAMELIST/DATA/ & -- \\
\hline CCORRT(NC) & $c_{t}(E q .6 .4 b)$ & NAMELIST/DATA/ & -- \\
\hline REYTRN(NC) & $\mathbf{R e}_{\mathrm{tr}}$ & NAMELIST/DATA/ & $\begin{array}{l}\text { Reynolds no. for laminar/ } \\
\text { turbulent transition }\end{array}$ \\
\hline REYLEN & $\mathbf{d}$ & NAMELIST/DATA/ & $\begin{array}{l}\text { Reference length for } \\
\text { Reynolds no. }\end{array}$ \\
\hline $\left.\begin{array}{l}\text { XFOR } \\
\text { YFOR } \\
\text { ZFOR }\end{array}\right\}$ & $\begin{array}{l}\text { Force-structure } \\
\text { location }\end{array}$ & $\begin{array}{l}\text { Force-structure } \\
\text { specification } \\
\text { records }\end{array}$ & $\begin{array}{l}\text { For direction and location, } \\
\text { see Sec. } 6.4\end{array}$ \\
\hline
\end{tabular}

a NF is a force-structure number; NC is a correlation number. 
Table 12. Input variables related to tube-side force-structure modeling

\begin{tabular}{clll}
\hline $\begin{array}{c}\text { FORTRAN } \\
\text { Name }\end{array}$ & $\begin{array}{c}\text { Description of } \\
\text { Variable }\end{array}$ & $\begin{array}{c}\text { COMMIX Input } \\
\text { Section }\end{array}$ & Remarks \\
\hline ATBL & $a_{\ell}$ (Eq. 6.4a) & NAMELIST/TUBS/ & - \\
BTBL & $b_{\ell}$ (Eq. 6.4a) & NAMELIST/TUBS/ & - \\
CTBL & $c_{\ell}$ (Eq. 6.4a) & NAMELIST/TUBS/ \\
ATBT & $a_{t}$ (Eq. 6.4b) & NAMELIST/TUBS/ & - \\
BTBT & $b_{t}$ (Eq. 6.4b) & NAMELIST/TUBS/ \\
CTBT & $c_{t}$ (Eq. 6.4b) & NAMELIST/TUBS/ \\
RETRN & RETR & NAMELIST/TUBS/ & - \\
DMTB & $D_{\ell}$ (Eq. 6.3) & NAMELIST/TUBS/ & Tube inside surface \\
FORCTB & $C_{1}$ (Eq. 6.3) & NAMELIST/TUBS/ & - \\
\hline
\end{tabular}<smiles>[R20]CC([R20])[R20][O-]</smiles>

where XFOR, YFOR, or ZFOR are to input the direction of force. The variable NF stands for the force-structure number, and IB .... KE stands for the beginning and end of the $\mathrm{I}, \mathrm{j}, \mathrm{k}$ indices of the cell locations. For more details relating to force-structure location input, see Appendix A.

\subsection{Modeling Recommendations}

\subsubsection{Staggored-Grid System}

In the finite-volume formulation, the frictional resistance due to a solid structure in a flow domain is considered an additional source term in the momentum equation. Because a staggered-grid system is used in COMMIX-PPC, the control volumes for the momentum equations are displaced from the mass or energy cells, as illustrated in Fig. 28. Therefore. for modeling of the resistance term, it is important to remember that

- The distributed-resistance source term is for the staggered control volume, shown in Fig. 28, and

- The reference velocity used in the resistance source term equation is the velocity at the face of a cell. 


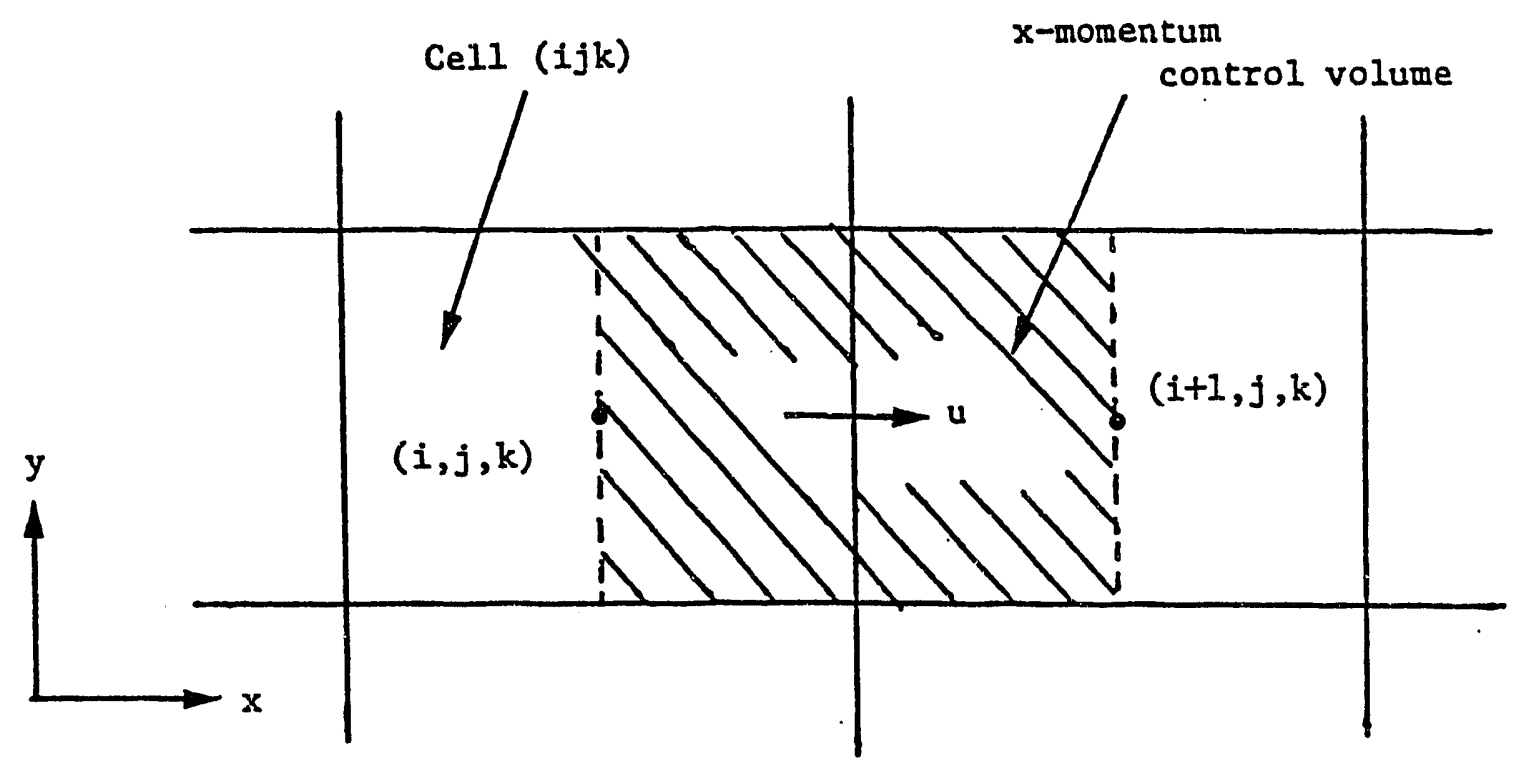

Fig. 28. $x$-Momentum control volume in a staggered-grid system

To illustrate this point, we consider the case of a sudden enlargement, as shown in Fig. 29. The pressure loss due to an abrupt change in area is generally expressed in terms of a loss coefficient $K_{1}$ or $K_{2}$, depending on which reference velocity is used,

$$
\begin{aligned}
\Delta p & =K_{1} \frac{1}{2} \rho V_{1}^{2} . \\
& =K_{2} \frac{1}{2} \rho V_{2}^{2} .
\end{aligned}
$$

where subscripts 1 and 2 refer to smaller and larger cross sections, respectively. In such cases, the user must look at the location of abrupt expansion (see Fig. 29) and then prescribe a suitable correlation for the loss coeficient $K_{1}$ or $K_{2}$.

\subsubsection{Friction-Factor Library}

Occasionally, the COMMLX-PPC user may find that the desired correlation is not of the form prescribed in Sec. 6.2. When this occurs, the user may

- Approximate the correlation to conform with the input form, or

- Implement a new correlation in the code.

The user who wishes to implement a new correlation should first examine the subroutine FORCES to see what correlation numbers are free and available. Then, with other library correlations as a guide, a new correlation can be inserted appropriately in subroutine FORCES and recompiled.

\subsubsection{List of Correlations}

To save the user's time in searching the vast literature, a set of correlations commonly needed by COMMLX-PPC users has been complled and listed in Appendix C. These correlations are not necessartly ideai; therefore, we welcome feedback and comments from all users so that an improved and expanded list can be prepared. 

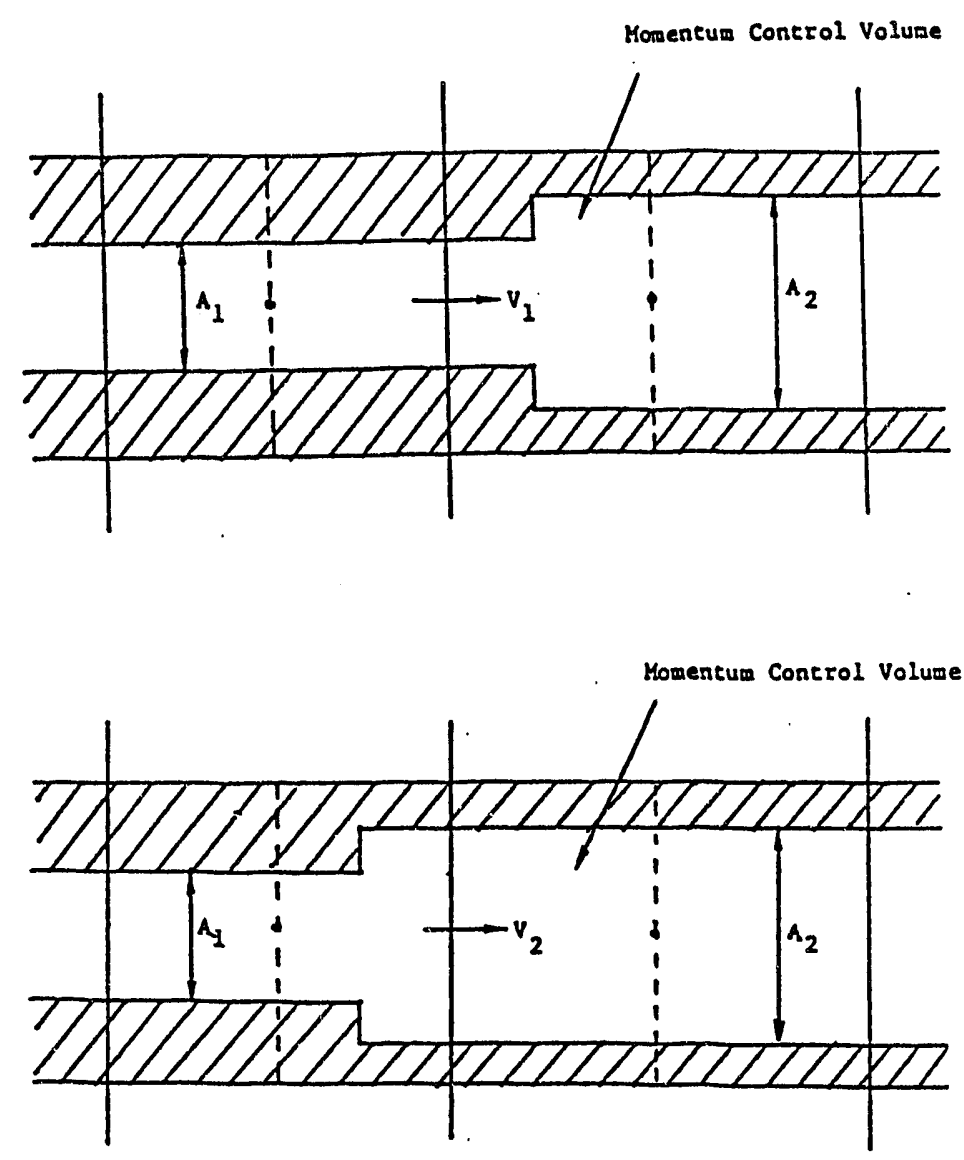

Fig. 29. Sudden enlargement

\section{Solution Procedure}

\subsection{Introduction}

As described in Vol. I, COMMIX-PPC employs the fully implicit scheme for the general finite-volume equations. Also available in COMMIX-PPC are the three matrix solvers for the scalar transport equations (energy, $k$, and $\varepsilon$ ) and the pressure equation. These options make the numerical computation more efficient for a given problem. In this section, we describe how to use these options and provide some guidance on the selection of the method that is suitable for a given application. Convergence and relaxation parameters are also discussed.

\subsection{Solution Methods}

Two variables in the solution procedure are given in Table 13. The user can choose one of the matrix solvers for the scalar transport equations and the pressure equation. The successive overrelaxation (SOR) method and the direct matrix inversion method (DMIM) are suitable for both the symmetric and nonsymmetric matrix and therefore can be selected 
Table 13. Variables in the COMMLX NAMELIST/GEOM/ Input Section of the solution procedure

\begin{tabular}{|c|c|c|}
\hline $\begin{array}{l}\text { FORTRAN } \\
\text { Name }\end{array}$ & Description & Remarks \\
\hline ISOLVE & $\begin{array}{l}\text { Flag to choose matrix solver for scalar transport } \\
\text { equations (energy, } k, \varepsilon \text {, and mass fraction } x \text { ) }\end{array}$ & $\begin{array}{l}=0 \text { for } \text { SOR }^{\mathrm{a}} \\
=1 \text { for } \mathrm{DMIM}^{\mathrm{b}}\end{array}$ \\
\hline ISOLVR & $\begin{array}{l}\text { Flag to choose matrix solver for the pressure } \\
\text { equation }\end{array}$ & $\begin{array}{l}=0 \text { for SOR } \\
=1 \text { for DMIM } \\
=11 \text { for PCGC }\end{array}$ \\
\hline
\end{tabular}

asOR: successive overrelaxation method.

bDMIM: direct matrix inversion method.

CPCG: Preconditioned conjugate gradient method.

for both the scalar transport equations and the pressure equation. The preconditioned conjugate gradient (PCG) method is applicable to symmetric matrices and is therefore suitable for the pressure equation only. The FORTRAN name ISOLVE is the flag for choosing the matrix solver for the scalar transport equations. If ISOLVE $=0, S O R$ is selected: if ISOLVE = 1, DMIM is selected. The FORTRAN name ISOLVR is the flag for choosing the matrix solver for the pressure equation. ISOLVR $=0,1$, and 11 corresponds to matrix solvers SOR. DMIM, and PCG, respectively. If SOR is selected, the user should specify the convergence parameter epsilon and the relaxation parameter omega, which will be separately discussed in Secs. 7.3 and 7.4. If the DMIM is selected, the user should input the parameters NNZERO and NSPACE in the NAMELIST/GEOM/. The values of these two parameters are not known a priori. A common approach is to input some arbitrary values for NNZERO and NSPACE, and submit a job. The output will then indicate the proper values to use for NNZERO and NSPACE. This procedure may have to be repeated several times.

In general, DMIM is most efficient if the number of the computational cells is less than 1000. If the number of computational cells is greater than 2000, the SOR method should be selected for the scalar transport equations and the PCG method for the pressure equation. If the number of computational cells is between 1000 and 2000 , there is no clear advantage in using one method over the others and the selection is problem-dependent.

\subsection{Convergence Parameters}

A list of convergence criteria employed in COMMIX-PPC is presented in Table 19 of Vol. I. The user must input these convergence parameters for the iterative solution schemes such as the SOR method and the PCG method. The list is repeated in Table 14 and all parameters shown are required as input. The default value for each parameter should be in the NAMELIST/DATA/ section of the input. 
Table 14. Convergence parameters

\begin{tabular}{cclc}
\hline $\begin{array}{c}\text { FORTRAN } \\
\text { Name }\end{array}$ & Symbol & \multicolumn{1}{c}{ Description } & $\begin{array}{c}\text { Default } \\
\text { Value }\end{array}$ \\
\hline EPS1 & $\varepsilon_{1}$ & $\begin{array}{l}\text { Mass convergence for pressure } \\
\text { equations }\end{array}$ & $10^{-4}$ \\
EPS2 & $\varepsilon_{2}$ & $\begin{array}{l}\text { Mass convergence for pressure } \\
\text { equations } \\
\text { All transport vartables }(h, k, e .\end{array}$ & $10^{-6}$ \\
EPS3 & $\varepsilon_{3}$ & $\begin{array}{l}\text { v, and w) } \\
\text { Turbulence parameters } k \text { and } \varepsilon\end{array}$ & $10^{-5}$ \\
EPS6 & $\varepsilon_{6}$ & & $10-5$ \\
\hline
\end{tabular}

\subsection{Relaxation Parameters}

The finite-volume discretization equations in COMMIX-PPC have been so constructed that, in the absence of interlinkages and nonlinearities, convergence will be certain. However, because the equations of interest are nonlinear and interlinked, care must be exercised to prevent divergence. One simple strategy is to slow down the changes that occur from iteration to iteration. This is accomplished by underrelaxation.

\subsubsection{Implicit Underrelaxation}

The general finite-volume discretization equation of COMMIX-PPC can be expressed by

$$
a_{0}^{\phi} \phi_{0}=\sum_{\ell} a_{\ell}^{\phi} \phi_{l}+b_{0}^{\dagger} \text {. }
$$

where the subscript $\ell$ denotes neighboring points.

We define

$$
\phi_{0}^{\text {new }}=\omega \phi_{0}+(1-\omega) \phi_{0}^{\circ} .
$$

where $w$ is the underrelaxation factor, $\phi_{0}$ satisfies Eq. 7.1, and $\phi_{0}^{\circ}$ is the most recent iteration value of $\phi_{0}$. Multiplying Eq. 7.2 by $\left(a_{0}^{\phi} / \omega\right)$ gives

$$
\left(\frac{a_{0}^{\phi}}{\omega}\right) a_{0}^{\text {new }}=a_{0}^{\phi} \phi_{0}+(1-\omega)\left(\frac{a_{0}^{\phi}}{\omega}\right) \text {. }
$$

Substituting Eq. 7.1 into Eq. 7.3 gtves

$$
\left(a_{0}^{\dagger} / \omega\right) \phi_{0}^{\text {new }}=\sum_{l} a_{l}^{\phi} \phi_{l}+b_{0}^{\dagger}+(1-\omega)\left(a_{0}^{l} / \omega\right) \phi_{0}^{\dot{0}} \text {. }
$$




$$
\left(a_{0}^{\dagger} / \omega\right) \phi_{0}^{\text {new }}=\sum_{l} a_{l}^{i} \phi_{l}+b_{0}^{\dagger}+(1-\omega)\left(a_{0}^{\dagger} / \omega\right) \phi_{0}^{*} .
$$

When $\phi_{0}$ tends to equal $\dot{\phi}_{0}$ (i.e., the iterations converge), Eq. 7.4 tends to become identical to Eq. 7.1. However, when the relaxation factor $\omega$ is less than 1, Eq. 7.4 will have a tendency to keep the resulting $\phi_{0}^{\text {nov }}$ closer to $\dot{\phi}_{0}^{\circ}$ than would Eq. 7.1. A value of $\omega$ close to zero will result in very heavy underrelaxation.

For the velocity components, a conservative value of $\omega=0.8$ may be used. Similar values may be used for turbulence kinetic energy and turbulence dissipation. A proper set of $\omega$ values can usually be obtained through working experience with a given class of problems. In COMMIX-PPC, the input parameters OMEGAV, OMEGAK, and OMEGAD are provided for implicit underrelaxtion for velocities $u, v$, and $w$, turbulence kinetic energy $k$, and turbulence dissipation rate $\varepsilon$, respectively. Table 15 lists the underrelaxation parameters and default values employed in COMMIX-PPC. It includes two additional underrelaxation parameters, OMEGAR for density and OMEGAT for turbulent viscosity. The defauil value for these two parameters is 1.0 .

\subsubsection{Successive Overrelaxation}

When the SOR method is used for the pressure equation and/or the scalar transport equation. the recommended default values for these parameters are those given in Table 16.

Table 15. Unclerrelaxation parameters and default values employed in COMMLX-PPC

\begin{tabular}{|c|c|c|}
\hline $\begin{array}{c}\text { FORTRAN } \\
\text { Name }\end{array}$ & $\begin{array}{l}\text { Default } \\
\text { Value }\end{array}$ & Remarks \\
\hline OMEGAV & 0.8 & Velocity components \\
\hline OMEGAK & 0.7 & Turbulence kinetic energy \\
\hline OMEGAD & 0.7 & $\begin{array}{l}\text { Rate of dissipation of } \\
\text { turbulence kinetic energy }\end{array}$ \\
\hline OMEGAR & 1.0 & Density \\
\hline OMEGAT & 1.0 & Turbulent viscosity \\
\hline
\end{tabular}

Table 16. Relaxation parameters and default values when SOR method is employed in COMMLX-PPC

\begin{tabular}{lcc}
\hline $\begin{array}{c}\text { FORTRAN } \\
\text { Name }\end{array}$ & $\begin{array}{c}\text { Default } \\
\text { Value }\end{array}$ & Remarks \\
\hline OMEGA & 1.5 & Pressure \\
RELAXK & 0.8 & Turbulence kinetic energy \\
\hline
\end{tabular}




\section{Auxiliary Input}

In addition to geometry specification, initialization, and model input, several auxiliary inputs are required. These inputs are briefly described here; detailed information is presented in Appendix A.

\subsection{Heat Transfer Correlation}

Relevant heat transfer correlations are needed to calculate the surface heat transfer coefficient in thermal-structure models. Currently, three types of heat transfer correlations are avallable in COMMLX-PPC. The heat transfer coefficient is linked to the thermal-structure model through the variable IHT in the thermal-structure prototype records NAMELIST/F/. Three input parameters are associated with a particular IHT:

- IHTCOR(IHT) Heat transfer correlation number,

- HTCLEN(IHT) Characteristic length, and

- HTCMUL(IHT) Heat transfer coefficient multiplier.

For example, if IHT $=2$, then

$$
\begin{aligned}
& \operatorname{IHTCOR}(2)=8, \\
& \text { HTCLEN(2) }=2.0, \\
& \text { HTCMUL(2) }=3.0,
\end{aligned}
$$

meaning that heat transfer correlation number 8 will be used for this particular thermal structure, the characteristic length used in the calculation of the Reynolds and Nusselt numbers is 2.0 meters, and the calculated heat transfer coefficient is multiplied by a factor of three.

The three types of heat transfer correlations in COMMLX-PPC are

1. If IHTCOR = $1-10$ :

$$
h=h_{0} \times N u \times k / d
$$

where ho $=$ heat transfer coefficient multiplier (HTCMUL),

$$
\begin{aligned}
\mathrm{Nu} & =\mathrm{C}_{1}+\mathrm{C}_{2} \operatorname{Re}^{\mathrm{C}_{2}} \mathrm{Pr}^{\mathrm{C}_{4}}, \\
\mathrm{Re} & =\mathrm{Reynolds} \text { number, } \\
\mathrm{Pr} & =\text { Prandtl number, } \\
\mathbf{k} & =\text { thermal conductivity, and } \\
\mathrm{d} & =\text { characteristic length (HTCLEN) }
\end{aligned}
$$

2. If IHTCOR = $11-20$ :

$$
h=h_{0}\left(C_{1}+C_{2} \operatorname{Re}^{C_{3}} \operatorname{Pr}_{4}\right)
$$


The constants $C_{1}, C_{2}, C_{3}$, and $C_{4}$ are the user input coefficients, and the FORTRAN names for these coefficients are HEATC1, HEATC2, HEATC3, and HEATC4, respectively. All input parameters for the heat transfer coefficient calculations should be in the NAMELIST/DATA/ section.

3. If IHTCOR $=21$ to 40 , the condensation heat transfer correlations are used. Values of IHTCOR from 21 to 30 are reserved for shell-side heat transfer correlations and values of IHTCOR from 31 to 40 are reserved for tube-side heat transfer correlations.

a) Shell-Side Heat Transfer Correlations

At the present time, only one value of IHTCOR is avallable, 1.e., IHTCOR $=21$. The condensation correlations of Nusselt (Eq. 9.5 of Vol. I) and Berman and Fuks (Eq. 9.4 of Vol. I), which account for the effect of entrained air, are used. The effects due to inundation, subcooling, and vapor shear are specified by the variables INNUDA, ISBUC, and ISHEAR, respectively, in NAMELIST/GEOM/.
INNUDA $=0$ inundation is not considered
1 Eq. 9.8 of Vol. I is used
ISUBC $=0$ subcooling is not considered
1 Eq. 9.9 of Vol. I. is used
2 Eq. 9.10 of Vol. I is used

ISHEAR = 0 effect due to vapor shear is not considered

1 Eq. 9.11 of Vol. I is used

b) Tube-Side Heat Transfer Condition

The heat transfer correlation for tube-side flow (single-phase) has the same form as in Eq. 8.1 for which the Nusselt number is given by Eq. 8.2. If IHTCOR $=31$, the Dittus-Boelter correlation for $\mathrm{Nu}$ is used.

The effect due to biofouling is specified by the variable IFOUL in NAMELIST/GEOM/.

IFOUL $=0$ biofouling effect is not considered

1 biofouling effect is considered.

When IFOUL $=1$, the fouling resistance, FOUL, must be specified in NAMELIST/DATA/.

Other heat transfer correlations may be selected by the user if deemed appropriate. The new additions can be defined by using an IHTCOR number greater than 41 . They should be inserted in the subroutine HTCOEF and recompiled.

\subsection{Fluid and Material Properties}

Fluid properties are determined by the variable MATYPE in the input section of NAMELIST/DATA/. The values of MATYPE may be any integer from 1 to 22 . When the 
value of MATYPE is from 1 to 20, the fluid properties are computed from the simplified equations. If MATYPE is 21 or 22 , the fluid properties are calculated by rigorous subroutines for the following:

The simplified equations for enthalpy $h$, density $\rho$, thermal conductivity $\lambda$, viscosity $\mu$. saturation pressure $P_{\text {sat, }}$ and molecular diffustvity $D$ are

$$
\begin{aligned}
h\left(\frac{J}{k g}\right) & =C_{0}^{h}+C_{1}^{h} T+C_{2}^{h} T^{2}+C_{3}^{h} P \\
\rho\left(\frac{k g}{m^{3}}\right) & =C_{0}^{p}+C_{1}^{p} T+C_{2}^{p} P /(T+273.16) \\
k\left(\frac{w}{m-{ }^{\circ} C}\right) & =C_{0}^{k}+C_{1}^{k} T+C_{2}^{k} T^{2} . \\
\mu(P a \cdot s) & =C_{0}^{\mu}+C_{1}^{\mu} T+C_{2}^{\mu} /(T+273.16) \\
P_{s a t}(P a) & =\exp \left(C_{0}^{p}+C_{1}^{p} T+C_{2}^{P} T^{2}\right) \\
D\left(\frac{m^{2}}{s}\right) & =\left(C_{0}^{d}+\frac{C_{1}^{d}}{p}\right)(T+273.15)^{C_{2}^{d}}
\end{aligned}
$$

where $\mathrm{T}$ is in ${ }^{\circ} \mathrm{C}$ and $\mathrm{P}$ is in $\mathrm{Pa}$. For each property, the user must input the constants $\mathrm{C}_{0}$. $C_{1}$, etc. The FORTRAN names for these constants are
$\mathrm{C} \oslash \mathrm{H}, \mathrm{C} 1 \mathrm{H}, \mathrm{C} 2 \mathrm{H}, \mathrm{C} 3 \mathrm{H}$
Coefficients in enthalpy equation. CORO, C1RO, C2R Coefficients in density equation. CØK, C1K, C2K Coefficients in conductivity equation, CØMU, C1MU, C2MU Coefficients in viscosity equation.
CØP, C1P, C2P Coefficients in saturation pressure equation, and COD, C1D, C2D Coefficients in molecular diffusivity equation.

Linkage of the material properties to the thermal-structure model is through the variable MI in the thermal-structure prototype records NAMELIST/M/. For example, if MI $=2$ for a given material and

$$
\begin{aligned}
& C \varnothing \mathrm{K}=20.0,40.0,60.0 . \\
& \mathrm{C} 1 \mathrm{~K}=10^{-3}, 10^{-4}, 10^{-5} .
\end{aligned}
$$

the thermal conductivity of the material is given by

$$
k=40+10^{-4} \times T, \text { in } W / m-{ }^{\circ} \mathrm{C} .
$$

Similarly, if MATYPE $=1$, the fluid properties are calculated by

$$
k=20+10^{-3} \mathrm{~T}, \text { in } \mathrm{W} / \mathrm{m}-{ }^{\circ} \mathrm{C} \text {. }
$$




\subsection{Turbulence Modeling}

In COMMIX-PPC, two options are provided to account for the effect of turbulent flow:

- Constant-diffusivity model, and

- $k-\varepsilon$ two-equation turbulence model.

The theory and equations relating to these models are described in Vol. I. Here, we present only the information needed by the user.

\subsubsection{Signal Paramoter}

The variable ITURKE in NAMELIST/GEOM/ specifies which turbulence model is to be used:

$$
\begin{aligned}
\text { ITURKE } & =0 \text { Use the no-turbulence (laminar flow) or constant-diffusivity model, } \\
& =12 \text { Use the } k-\varepsilon \text { two-equation turbulence model. }
\end{aligned}
$$

\subsubsection{Constant-Diffusivity Modol}

In the constant-diffusivity model (ITURKE $=0$ ), the user must specify the values of the turbulent viscosity ( $\left.\mu_{\text {tur }}\right)$ and turbulent conductivity $\left(k_{\text {tur }}\right)$ in NAMELIST/DATA/. The corresponding FORTRAN variables are TURBV and TURBC, respectively.

It is recommended that whenever possible, the prescribed values be based on experimental data. If experimental data are not avallable, a user can provide his/her own estimate or use the procedure suggested in Sec. 7.1 of Vol. I.

\subsubsection{K-E Two-Equation Turbulence Model}

In the two-equation model (ITURKE = 12). the transport equations of the turbulence kinetic energy $k$ and its dissipation rate are solved. As has been described in Vol. I, eight presumably constant coefficients are employed in the $k-\varepsilon$ two-equation turbulence model. Table 17 is a list of the FORTRAN names and default values of these coefficients. These parameters are required only if the user wishes to use values different from the default values gtven in the table, in which case, the values desired by the user should be in the input section of NAMELIST/DATA/.

Table 18 lists additional inputs that the user may need for the $k-\varepsilon$ two-equation turbulence model. These parameters have already been described in previous sections. For example, the relaxation parameters are explained in Sec. 7.4, and the boundary turbulence parameters (TKB and TDB) are explained in Sec. 4.2. They are presented here mainly for cross reference.

All parameters listed in Table 18, with the exception of TKB and TDB, should be in the input section of NAMELIST/DATA/. TKB and TDB should be in the surface-element initialization records. 
Table 17. Coefficients employed in $k-\varepsilon$ twoequation turbulence model

\begin{tabular}{lcc}
\hline $\begin{array}{l}\text { FORTRAN } \\
\text { Name }\end{array}$ & $\begin{array}{c}\text { Symbols Used } \\
\text { in Volume I }\end{array}$ & $\begin{array}{c}\text { Default } \\
\text { Value }\end{array}$ \\
\hline PRNDLH & $\sigma_{\mathrm{h}}$ & 0.9 \\
PRNDLK & $\sigma_{\mathrm{k}}$ & 1.0 \\
PRNDLD & $\sigma_{\mathrm{z}}$ & 1.3 \\
CT1 & $\mathrm{C}_{1}$ & 1.44 \\
CT2 & $\mathrm{C}_{2}$ & 1.92 \\
CDTURB & $\mathrm{C}_{\mathrm{D}}$ & 0.09 \\
AKAPPA & $\mathrm{K}$ & 0.42 \\
EE & $\mathrm{E}$ & 9.0 \\
\hline
\end{tabular}

Table 18. Possible additional input for $k-\varepsilon$ two-equation turbulence model

\begin{tabular}{|c|c|c|}
\hline Variable & Function & $\begin{array}{l}\text { Default } \\
\text { Value }\end{array}$ \\
\hline ITMAXK & Maximum number of iterations for $k$ equation & 29 \\
\hline OMEGAT & Relaxation factor for turbulent viscosity & 1.0 \\
\hline OMEGAK & Relaxation factor used in the solution of $k$ equation & 0.7 \\
\hline OMEGAD & Relaxation factor used in the solution of $\varepsilon$ equation & 0.7 \\
\hline RELAXK & Relaxation factor for $\mathbf{k}$ & 0.8 \\
\hline EPS6 & Convergence criterion for the $k$ and $\varepsilon$ equations & $10^{-5}$ \\
\hline TKB & Boundary turbulence kinetic energy & $10^{-16}$ \\
\hline TDB & $\begin{array}{l}\text { Dissipation rate of boundary turbulence kinetic } \\
\text { energy }\end{array}$ & $10^{-10}$ \\
\hline
\end{tabular}

\subsection{Time-Step Size}

COMMIX performs thermal-hydraulic calculations by marching in time. All values of the dependent variables at a given time $\mathbf{t}$ and the corresponding time step $\mathbf{n}$ are known. The values of the dependent variables at time $t+\Delta t$ and time step $n+1$ are calculateu. By repeating this procedure, the thermal-hydraulic conditions are determined for the desired time span.

For steady-state calculations, the same procedure is followed. We start with an initial state and continue the marching-in-time process until the values of all dependent variables slowly vary. The size of the time step for the implicit-steady-state calculation can be very large, e.g., as much as 10-20 times the Courant time-step criterion. 
In COMMIX-PPC, there are two options for selecting the time-step size:

- The user can prescribe the desired time-step size as input; details of this option are given in Appendix A.

- The automatic time-step option can be used.

In the automatic time-step option, the time-step size is evaluated on the basis of the Coisrant condition:

$$
\Delta t=C_{1} \Delta t_{C}
$$

where $C_{1}$ is the user-prescribed coefficient and $\Delta t_{c}$ is the time-step size evaluated from the Courant condition. The Courant time-step size is the minimum time required for fluid to be convected through a cell. The automatic time-step option does not provide an optimum time-step size for a given transient condition. Additional work is needed to develop an automatic time-step selection scheme, where an optimum time-step size is selected during the course of a transient calculation, as described in Vol. I.

The following input variables are related to time-step size:

IDTIME Signals whether the time-step is user-specified or a Courant time-step.

$\left.\begin{array}{l}\text { DT(1) } \\ \text { DT(2) } \\ \text { LASTDT }\end{array}\right\} \begin{aligned} & \text { DT(1) is the time-step size for Steps 1-LASTDT. } \\ & \text { DT(2) is the time-step size for steps after LASTDT. }\end{aligned}$

RDTIME Factor $C_{i}$ is used for multiplying the Courant time-step size (Eq. 8.10).

Several other time-related parameters are listed in Table 19. These parameters provide the user with additional flexibility in controlling how a particular run should be conducted. For example, if the user wants to run for just one time step (for debugging), the parameter NTMAX should be set to 1 . If the user wants to run a particular problem for just $1 \mathrm{~min}$, the parameter TIMAX should be set to 60 .

\subsection{Output}

An input procedure, described in Appendix A, is provided for printing array values of a range of variables at given locations (specified plane) and at a given time or time step.

\section{Steady-State Calculation}

\subsection{Introduction}

In COMMIX-PPC, even when analyzing a transient problem, we must generate an initial condition for the transient anaiysis by first obtaining a steady-state solution. 
Table 19. Time-related parameters

\begin{tabular}{llc}
\hline Name & \multicolumn{1}{c}{ Description } & $\begin{array}{c}\text { Default } \\
\text { Value }\end{array}$ \\
\hline NTMAX & Maximum time step for a gtven run & 99999 \\
TIMAX & Maximum time for a given run & $3.6 \times 10^{7}$ \\
ITEMER & $=0$, no subroutine time information shown \\
& $=1$, subroutine time information shown at the \\
& end of a run \\
& 2, subroutine time information shown after every \\
& call & 0 \\
TREST & $\begin{array}{l}\text { If the elapsed run time exceeds TREST, the run } \\
\text { is terminated after cptionally writing a restart file }\end{array}$ & 3600 \\
TSTART & $\begin{array}{l}\text { This value should be reset to zero at the beginning } \\
\text { of a transient run (STATE = 2) }\end{array}$ & 0.0 \\
\hline
\end{tabular}

To perform a steady-state calculation, the problem is treated as if it were a transient case. We prescribe an estimated distribution of the dependent variables as their initial values and continue marching in time until the distribution slowly varies and satisfies the prescribed convergence criteria.

\subsection{Input Preparation}

To start a steady-state calculation, we prescribe

- $\quad$ Control flag ISTATE $=0$.

- Control flag IFRES $=1$.

The control flag IFRES $=1$ implies that we are starting a new case and that at the end of the run we want the results to be written on a restart file. If we do not desire a restart file to be written, we prescribe IFRES $=0$. For continuation of a steady-state run, we spectfy

- $\quad$ ISTATE $=1$ and

- IFRES $=3$

in the input of the continuation run.

- Geometrical information.

- Constant-value boundary conditions (use the surface-element initialization records if the boundary conditions are nonuniform). 
- The best estimates for the initial values of all dependent variables; to save computer running time, it is recommended that prescribed initial estimated values be as close as possible to the expected solution.

- Number of iterations $I T=1$. Because we are performing a steady-state simulation, each time step represents a steady-state iteration; therefore, more iterations per time step are not required.

- Time-step size DT can be large. We recommend starting with DT $=1000 \mathrm{~s}$ or larger. If this causes a convergence problem, it then can be reduced to. for example. 100 or $10 \mathrm{~s}$ or even less.

- Fluid and material properties.

- Other ancillary information, e.g., output printing desired.

- Force-structure input. if any.

- Thermal-structure modeling with heat source, if any. For a steady-state calculation, only the thermal structures with heat sources need to be considered.

- Turbulence-model input, if necessary.

\subsection{Steady-State Convergence Criteria}

We define a steady-state solution as having been achievea when the following criteria are satisfied simultaneously:

$$
\begin{gathered}
\left(\frac{|\Delta h|}{h}\right)_{\max }<\varepsilon_{3}, \\
\left(\frac{|\Delta u|}{V E L M A X}\right)_{\max }<\varepsilon_{3}, \\
\left(\frac{|\Delta v|}{V E L M A X}\right)_{\max }<\varepsilon_{3}, \\
\left(\frac{|\Delta W|}{V E L M A X}\right)_{\max }<\varepsilon_{3}, \text { and } \\
|\delta|_{\max }<\text { DCONV. }
\end{gathered}
$$

Here $\Delta h, \Delta u, \ldots .$, are the changes in enthalpy, velocity components, etc., between successive time steps, $\delta$ is the mass residue, $\varepsilon_{3}$ is the steady-state convergence criterion, VELMAX is the maximum of all velocity magnitudes, and DCONV is the mass convergence parameter calculated from

$$
\operatorname{DCONV}=\varepsilon_{1}\left[\left(\frac{\rho \gamma_{1} u_{1}}{\gamma_{v} \Delta x_{1}}\right)_{\max }+\varepsilon_{2}\right] \text {, in } \mathrm{kg} / \mathrm{m}^{3}-s
$$


where $\varepsilon_{1}$ and $\varepsilon_{2}$ are the input convergence constants, subscript 1 stands for the three coordinates, $\gamma_{i}$ is the suface porosity, and $\gamma_{v}$ is the volume porosity. The default values for $\varepsilon_{1}, \varepsilon_{2}$, and $\varepsilon_{3}$ are $10^{-4}, 10^{-6}$, and $5 \times 10^{-5}$, respectively.

\section{Transient Calculations}

\subsection{Introduction}

In COMMLX-PPC, any one of the following conditions or their combination will result in a transient problem:

- Transient velocity boundary condition.

- Transient temperature and surface heat flux boundary conditions,

- Transient pressure boundary condition, and/or

- Transient heat source.

\subsection{Procedure}

The following procedure is recommended for running a transient problem:

- Obtain a steady-state solution using constant-value boundary conditions and guessed initial distributions of all dependent variables, and write the results on a restart file. The constant boundary values must be the values at time $t=$ 0 of the transient problem.

- Run the transient problem, using the restart data and the following additional input in NAMELIST/DATA/.

-ISTATE $=2$.

-TSTART $=$ starting time of the transient.

$-K F L O W(N)=100+N F$; this is for the transient velocity boundary condition. NF defines the transient function number to be used for the transient condition on surface $\mathbf{N}$.

$-\mathrm{KTEMP}(\mathrm{N})=100+\mathrm{NF}$ or $300+\mathrm{NF}$; this is for the transient temperature or heat flux boundary condition. NF defines the transient furction number to be used for the transient condition on surface $\mathrm{N}$.

-KPRES(N) = $100+N F$; this is for the transient pressure boundary condition. NF defines the transient function number to be used for the transient condition on surface $\mathbf{N}$. 
-NOFGT is the number of the transient function used as a multiplier of the specifled volumetric heat source (QSOU) distribution. For example, if NOFGT $=2$,

$$
Q(x, y, z, t)=f_{2}(t) x \operatorname{QSOU}(x, y, z),
$$

where $Q(x, y, z, t)$ is the transient volumetric heat source, $f_{2}$ is the transient function number 2 , and $\operatorname{QSOU}(x, y, z)$ is the spatial heat source distribution in the fluid.

-TVAL represents the values of the independent variable (time) of the transtent functions.

-FVAL represents the values of the dependent variable of the transient functions.

$-\mathrm{NEND}(\mathrm{N})$ is the number of points used to prescribe the transient function number $\mathbf{N}$.

- Other ancillary information - time-step size, output, etc.

\subsection{Transient Functions}

In COMMIX-PPC, we use the relation

$$
F(t)=F(0) * f(t)
$$

to prescribe the desired variation of a function with time. Here. $F(0)$ is the value of a function at time $t=0$ and $f(t)$ is a dimensionless transient function. The following is information relevant to the use of $\mathrm{f}(\mathrm{t})$ in COMMIX.

- A set of $f$ and $t$ values must be prescribed for each transient function. Cubic spline-fit coefficients are evaluated to approximate a transient function as a polynomial.

- Up to 25 functions, consisting of up to 100 points, can be defined.

- All transient functions should be normalized with respect to values at time $t$ $=0$.

- FVAL and TVAL are the names of FORTRAN variables that prescribe discrete values of $f$ and $t$, respectively.

- $\mathrm{NEND(NF)}$ is the number of discrete $f$ and $t$ values prescribed for transient function \#NF.

- FVAL and TVAL are one-dimensional arrays; the first value of the second transient function immediately follows the last value of the first function. The same pattern is followed for all subsequent transient functions.

- Discontinuities in a function can be indicated by specifying the same $t$ value twice for the same or different $f$ values. 


\subsection{Creation of a Load Module}

To ease the task of creating load modules (binary files) to fit the size of the problem being considered, a dynamic storage allocation scheme is implemented in COMMIX. Space for the geometry-dependent varlables is allocated in the varlable $S$ of COMMON/SPACE/. The address of each varlable is computed at the beginning of each run. These addresses are then passed into subroutines, where the varlables are named and varlably dimensioned.

\subsection{Input/Output}

\subsubsection{Input Data File 5}

The input for File 5 is described in Appendix A. The most recent version of the input description is generally distributed with the code. The input contains a mixture of NAMELIST and formatted data. It is rewound to allow for multiple passes through the fille. The user may wish to change the file number of the read statements if the user's system does not provide for rewinding of File 5.

\subsubsection{Printed Output File 6}

The printed output from COMMLX-PPC is written into File 6. A 133-character line length is assumed, with Column 1 being the carrlage control. The input data from File 5 and summary information, depending on the problem, are always printed. But the bulk of the output is user-specified and controlled by parameters such as ISTPR, NTHPR. NTPRNT, and TPRNT, which are described in Appendix A.

\subsubsection{Restart Information Files 9 and 10}

The restart capabilities of COMMIX-PPC are programmed in SUBROUTINE RESTAR. Blocks of information are written into File 10, which can be read by a subsequent job from File 9 to permit continued processing from the point at which the restart file was written.

Restart files are written optionally (see IFRES in Appendix A) in any of the following three events:

- Steady state is reached.

- The time specified for the job has elapsed.

- A specified time or time step has been reached.

The first event is indirectly controlled by the convergence parameters. The second and third events can be controlled by variables described in the "Restart Option" section under NAMELIST/GEOM/ and the "TIme- and Time-Step-Related Parameters" section under NAMELIST/DATA/ in Appendix A. 
After the restart information is written into File 10, several additional records are written. These records contain, in effect, a snapshot of the simulation as it existed when the restart was written. This information can then be used, by interfacing with the user's graphics programs, to obtain graphic plots, e.g., velocity vectors and isotherms.

\subsubsection{Plot Tape Flle 76}

Before reaching a steady state, it is generally adequate to obtain plots only for points in time at which restart files are written. However, once steady state has been reached and a driving transient has been turned on, it is often desirable to save the complete history of the flow and temperature fields. SUBROUTINE PLTAPE provides this optional capability (see NTPLOT in Appendix A). At the beginning of File 76, a group of records containing geometry and properties information is written. Then, at user-controlled time-steps, the entire velocity and temperature fields are written. This file can then be used to interface with the user's plotting routines. At the present time, the plotting routines for condenser modeling are avallable for shell-side flow only.

\subsection{Error Detection and Diagnostics}

It is generally impossible to anticipate all the potential error conditions that one might encounter while using COMMIX. However, an attempt has been made to provide information that will guide the user through abnormal terminations.

Key variables are tested for meaningful values in many places or where certain paths of a branch statement indicate error conditions. Some of these have been coded to call SUBROUTINES ERRORS and ERRCHK. These two subroutines print short error messages and determine whether processing can continue or must terminate.

The "Error Messages" section in Appendix A contains expanded explanations of the errors encountered.

\section{Global Mass and Enthalpy Balances}

\subsection{Global Mass Balance}

Shell side

The global balance equations for the mixture and for each component fluid are obtained by integrating Eqs. 2.1 and 2.2 of Vol. I over the entire flow domain; thus,

$$
\int_{V} \frac{\partial\left(\gamma_{v} \rho\right)}{\partial t} d v+\int_{A} \gamma_{A} \rho \bar{v} \cdot \overline{d A}=\int_{V} \gamma_{v} \dot{m}^{\prime \prime} d v
$$

for the mixture, and

$$
\int_{V} \frac{\partial\left(\gamma_{v} \rho x_{k}\right)}{\partial t} d v+\int_{A} \gamma_{A} \rho x_{k} \bar{v} \cdot \overline{d A}=\int_{V} \gamma_{v} \dot{m}_{k}^{\prime \prime \prime} d v
$$

for the component fluid. 
Equations 12.1 and 12.2 can be expressed as

$$
\left[\begin{array}{c}
\text { mass accumulation } \\
\text { rate }
\end{array}\right]=\left[\begin{array}{c}
\text { net mass flow convected } \\
\text { into system }
\end{array}\right]+\left[\begin{array}{c}
\text { mass generation } \\
\text { rate }
\end{array}\right] \text {. }
$$

By denoting MDASDT = mass accumulation rate, $\mathrm{kg} / \mathrm{s}, \mathrm{FLOSUM}=$ net mass flow convected into the system, $\mathrm{kg} / \mathrm{s}$, and XSOURC = mass generation rate, $\mathrm{kg} / \mathrm{s}$, the global mass in balance can be calculated from

$$
\Delta \dot{\mathrm{m}}=\text { DMASDT }- \text { FLOSUM }- \text { XSOURC. }
$$

Each term of the above equation is computed and printed in the shell-side global mass balance section of the printout when Subroutine OUTPUT is called.

Tube side

The global mass balance equation is obtained by integrating Eq. 8.1 over all the condenser tubes, i.e.,

$$
\int_{V}\left[\frac{\partial p}{\partial t}+\frac{\partial}{\partial x}(\rho u)\right] d v=0
$$

or

$$
\left[\begin{array}{c}
\text { net mass } \\
\text { accumulation rate }
\end{array}\right]=\left[\begin{array}{c}
\text { net mass flow convected } \\
\text { into condenser tubes }
\end{array}\right] \text {. }
$$

By denoting DMASDT = mass accumulation rate and FLOSUM = net mass flow convected into condenser tubes, the global mass imbalance $\Delta \dot{m}$ for the tube-side flow is given by

$$
\Delta \dot{\mathbf{m}}=\text { DMASDT }- \text { FLOSUM. }
$$

Each term of the above equation is computed and printed in the tube-side global mass balance section when Subroutine OUTPUT is called.

\subsection{Global Enthalpy Balance}

Shell side

The enthalpy imbalance can be determined from the following relation:

$$
\begin{aligned}
{\left[\begin{array}{c}
\text { net enthalpy } \\
\text { accumulation rate }
\end{array}\right]=} & {\left[\begin{array}{c}
\text { net enthalpy convected } \\
\text { into the system }
\end{array}\right]+\left[\begin{array}{c}
\text { net heat conducted } \\
\text { into the system }
\end{array}\right] } \\
& +\left[\begin{array}{c}
\text { net latent heat released } \\
\text { within the system }
\end{array}\right]+[\text { pressure work }] .
\end{aligned}
$$

In the COMMIX code, we define

DHNET = net enthalpy accumulation rate.

HINSUM = net enthalpy converted into the system.

gINSUM = net heat conducted into the system, 
QSOURC = net latent heat released within the system, and DPDT $=$ pressure work.

Therefore, the enthalpy imbalance is calculated from

$$
\Delta E=\text { DHNET - HINSUM - GINSUM - QSOURC - DPDT. }
$$

Each term in the above equation is computed and printed in the shell-side global enthalpy balance section of the printout when Subroutine OUTPUT is called.

Tube side

The global balance equation is obtained by integrating Eq. 8.3 over all the tubes. It can be expressed as

$$
\left[\begin{array}{c}
\text { net enthalpy } \\
\text { accumulation rate }
\end{array}\right]=\left[\begin{array}{c}
\text { net enthalpy convected } \\
\text { into condenser tubes }
\end{array}\right]+\left[\begin{array}{l}
\text { net heat transferred } \\
\text { to the cooling water }
\end{array}\right]
$$

In COMMIX-PPC, we define

$$
\begin{aligned}
\text { dHdT } & =\text { net enthalpy accumulation rate, } \\
\text { HINSUM } & =\text { net enthalpy converted into condenser tubes, } \\
\text { QSOURC } & =\text { net heat transferred to the cooling water. }
\end{aligned}
$$

and the energy imbalance is calculated from

$$
\Delta \mathrm{E}=\mathrm{dHdT}-\text { HINSUM }- \text { GSOURC. }
$$

Each term in the above expression is computed and printed in the tube-side global enthalpy balance section when Subroutine OUTPUT is called.

\section{Concluding Remarks}

The development of COMMIX began in 1976, with emphasis on the analysis of fuel assemblies in nuclear reactors, specifically under natural-circulation conditions. However, as application multiplied, demands increased, and the structure of COMMLX changed in response to users' needs, it became apparent that COMMIX could be made into a useroriented general-purpose code with a wide range of capabilities and applicabilities.

The development of COMMLX has been a continuous, dynamic process. New modifications and improvements are implemented continuously in response to

- New development of physical models and solution procedures.

- Feedback from users, and

- A desire to streamline the code. 1.e., make it more user-oriented, and increase its flexibility. 
The first version of COMMIX, named COMMLX-1, was released in March 1978. Development continued and many features were added, e.g.. thermat- and force-structure models, a cylindrical geometry option, semi- and fully implicit solution procedures, etc. In December 1983. COMMLX-1A was released. Further development led to COMMIX-1B, which was released in September 1985 and included new features such as turbulence models and a volume-weighted skew-upwind difference scheme. New matrix solvers, namely. DMIM and preconditioned conjugate gradient method, and modified finite volume formulation using the concept of volume averaged velocity, have been incorporated into COMMIX-1C, released in February 1990. Two more capabilities, namely, treating fluids of multicomponents and shell-tube thermal interaction, have been the most recent additions in COMMIX-PPC. These capabilities are introduced specifically for performance analysis of power plant condensers.

Since the inception of COMMIX, code verification has been performed in parallel with development. Extensive simulations have been performed to check and verify every step of the development. We belleve that COMMIX is a very well-tested code. However, because COMMIX is large and versatile, some bugs might still have been overlooked. We certainly welcome feedback and comments from any user so the code can be further improved.

Within the constraints of available time and manpower, we have made very effort to make COMMLX a well-tested, user-ortented computer code. No doubt, many more improvements could be implemented to make it even more user-oriented. The following work is in progress:

- Implementation of more error detection and diagnostics to provide information that will guide the user through abnormal terminations.

- Development of a software package for computing all geometry-related information (e.g., mesh size, volume and directional surface porosity, and surface area) and generating input for COMMIX.

- Development of an interactive input processor for auxiliary input information. e.g., boundary conditions, thermal structures, and force structures.

- Development of a postprocessor for connecting COMMIX output to plot processors for generating vector and isotherm plots.

\section{Acknowledgments}

This work is a result of international cooperation. The authors thank E. Lin, P. C. Chen, S. C. Cheng, Y. H. Cheng, T. K. Lee, C. K. Fwu, C. C. Jao, and M. W. Lin of Tatwan Power Company for supporting and sponsoring this work. We also thank Professors B. T. Chao and S. L. Soo of the University of Illinois at Urbana-Champaign for their stimulating discussions and constructive comments. 


\section{A.1 General Comments}

The units used are meter, kllogram, second, and degrees Celsius. These and other derived units are indicated after the description of variables requiring them.

Default values are indicated either by an asterisk or a value in parentheses after the variable description.

Arrays are indicated by the use of a subscript following the varlable name. The ranges of the subscripts are indicated in the following table. An asterisk in the 'Current Limit' column indicates that storage is allocated at execution time according to the value in the 'Range' column.

$\begin{array}{llr}\text { Index } & \text { Range } & \text { Current Limit } \\ \text { I } & \text { IMAX } & 99 \\ \text { IND } & \text { IMAX*JMAX } & \text { IND=I*(J-1)*IMAX } \\ \text { J } & \text { JMAX } & 100 \\ \text { K } & \text { KMAX } & 99 \\ \text { N } & \text { NSURF } & 99 \\ \text { NH } & \text { NHEATC } & 399 \\ \text { NM } & \text { Number of materials } & 10 \\ \text { NP } & & 24 \\ \text { NF } & \text { NFORCE } & 50 \\ \text { NC } & \text { NCORR } & \\ \end{array}$

\section{A.2 Some Terminology}

The computational area is partitioned into a number of computational cells, each bounded by consecutive X-, Y-, and Z-direction (or r-,0, and z-direction) grid planes. Surface (portions of a plane or cylinder) may be defined both on the exterior, bounding the computational area, and in the interior. The intersection of a surface and consecutive grid planes outlines a surface element. Surfaces which coincide with a grid plane are called regular surfaces, otherwise, they are called irregular surfaces. A regular cell is one with all faces coinciding with grid planes. Irregular cells have one irregular surface element.

\section{A.3 General Input Structure}

The user input is read from a file associated with unit 5. This input consists of two required namelists, optional namelists, and several other record groups.

The user may specify unit 5 input in any order. One possible order follows:

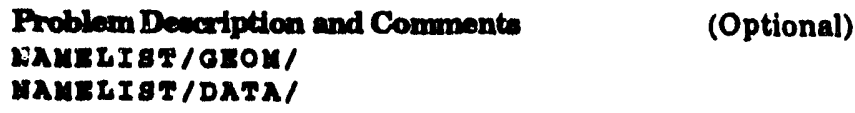


Boundery Burence Identificatton Reconds

Coll Inittalization Reconds

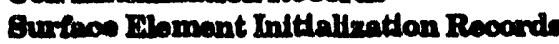

Toros Ebructure Epectitiontion Reconds

Theremal Etructurv Locotion Records

Tubo Eldo Desaription and Commenta

NAMIMUIST TUBS/

Condaner Tube Loontion Reconde
(Optional)

(Optional)

(Optional)

(Optional)

(Optional)

(Optional)

(Optional)

\section{A.4 Input}

\section{A.4.1 Problem Description and Comments}

In order to sense the presence of optional input, columns 1-4 of each line of input is compared with the list of key words listed below. When a match is found, the line is reread in the appropriate format. If no match is found, the line is ignored or, in effect, treated as a comment. Thus any number of user comments can precede NAMELISTS or be interspersed between non-NAMELIST input as long as column 1-4 do not contain any of the following keywords.

\begin{tabular}{|c|c|c|c|c|c|}
\hline REO & $\begin{array}{l}\text { IREO } \\
\text { YEOR }\end{array}$ & $\begin{array}{l}\text { IET } \\
\text { 2EOR }\end{array}$ & $\begin{array}{l}\text { TELB } \\
\text { AT }\end{array}$ & $\begin{array}{l}x \times s \\
s ?\end{array}$ & $\begin{array}{l}\text { XYSE } \\
\mathrm{GX}\end{array}$ \\
\hline Org & $I X$ & E. B & $\mathbf{P B}$ & QBA & RIB \\
\hline TIB & VIIBN & AI & AIX & $\mathbf{A} \mathbf{X}$ & AI \\
\hline 8I & 8 & Qs00 & TI & סL & VI \\
\hline nI & Fron & $\boldsymbol{T r} x$ & TD & TXB & TDB \\
\hline R2 & EXDO & POLD & TOB: & & \\
\hline
\end{tabular}

\section{A.4.2 NAMELIST/GEOM/}

The following variables are used to determine the amount of memory to be allocated for the run. It is important that they are specified correctly. The variables preceded by an asterisk can be approximated by a value larger than actually needed. The minimum values are printed after they are computed. Any of these varlables that remain unchanged for a subsequent restart run need not be respecified since they are read from the restart file.

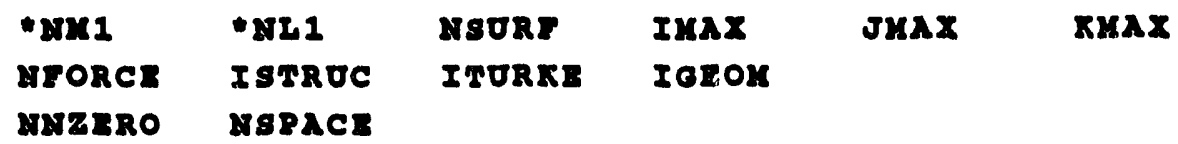

The following three varlables can be set to allow for more efficient computation when running one- or two-dimensional problems.
Irx 1 Perform all $x$-component calculations. (*)
0 Bypass $x$-component calculations.
IrY 1 Perform all y-component calculations. (*)
0 Bypass y-component calculations.
Irz 1 Perform all z-component calculations. (*)
0 Bypass z-component calculations.

IGzox 0 Regular Box geometry (Cartesian coordinate) option. (*) 
-1 Cylindrical geometry option using box geometry input.

Note 1 . A surface must be dedicated to $R=0.0$ when the origin is present. Set $\operatorname{xrLOW}(N)=-3$ and $\operatorname{xrzMP}(N)=400$ for that surface.

Note 2. For full $2.0^{*} \mathrm{PI}$ radian geometries $\mathrm{J}=1$ and $\mathrm{J}=\mathrm{JMAX}$ are automatically linked thus no surfaces need be defined at $Y=0.0$ and $Y=2.0 * \mathrm{PI}$.

$>0$ INOPERATIVE

I sorve Flag to determine the solution technique used to the transport equations. These include energy, turbulent kinetic energy and turbulent dissipation. (0)

0 Successtve Over-Relaxation (SOR) solution scheme used. Values for eps 1 and omega must also be specified when using this option.

1 The direct matrix inversion method (DMIM) solver is used. This option is recommended when the number of computational cells, $\mathrm{nm}$ l, is less than 1000. Values for nnzero and nspace are required when using this option. (*)

IsorvR Flag to determine the solution technique used to solve the pressure and component continuity equation. (0)

0 Successive Over-Relaxation (SOR) solution scheme used. Values for eps 1 and omega must also be specified when using this option.

1 The direct matrix inversion method (DMIM) solver is used. This option is recommended when the number of computational cells, $\mathrm{nm} 1$, is less than 1000. Values for nnzero and nspace are required when using this option. (")

11 A Pre-Conditioned Conjugate Gradient solver is used. This option is recommended when the number of computational cells, nml. is greater than 1000.

матот (1) Total number of components or materials.

MI 1

Total number of surface elements. (0)

$\mathbf{2} \mathbf{1}$

Total number of computational cells. (0)

Note. Both $\mathrm{NL1}$ and sac1 can be approximated by values larger than actually required. However, if this is done they must not be included in NamerIsT /OROx/ when restarting (ISTATz $>0$ ). Storage will be allocated according to the values specified in the input rather than the mininum storage needed. The minimum values are printed when computed. If one desires to change sin1 and/or arL1, it must be done only at the start a steady-state run $($ ISTATR $=0)$.

IXAx The maximum number of cells in the $x$-direction (R). (1)

Jux

The maximum number of cells in the $y$-direction (THETA). (1)

$\operatorname{sunx}$

The maximum number of cells in the z-direction. (1)

MSORF

The number of unique surfaces enclosing the computational domain. Unique surfaces are determined by a unique combination of the following three characteristics:

1. Velocity Boundary Condition

2. Temperature Boundary Condition

3. The unit normal vector to the surface.

$D X(I)$

The calculational cell sizes along the $x$-axis, $m$.

DY (J)

The calculational cell sizes along the $y$-axis, $m$ or rad.

Dz $(x) \quad$ The calculational cell sizes along the $z$-axis, $m$. 
The unit normal vectors referred to by the following three variables are those pointing into the computational domans.

zxORxL (x) The X-component of the unit normal vector to surface $\mathrm{N}$.

YwORxI (X) The Y-component of the unit normal vector to surface $N$.

zxorsz (x) The Z-component of the unit normal vector to surface $N$.

The required values for the following variables are internally computed during initialization for a problem. If the input values are too small, the run will terminate with a message indicating the proper size for these variables.

swszero Number of matrix non-zeros when using DMIM.

rspace Work space size when using DMIM.

ILMax Maximum number of surface elements on any surface.

ITORER In this version of COMMIX-PPC two turbulence models are included. For all of the details of input requirements for these options see the Turbulence Models in MAMBIIST/DATA/.

0 Constant Turbulent Viscosity Model (").

12 Two-Equation Turbulence Model.

IXPRNT 0 Cell number and surface number arrays are not printed. (*) Specifying LUPRNT $=1$ or LUPRNT $=2$ causes excessive geometry debugging information to be printed and execution to terminate. This information is of little use to the casual user and is not recommended.

1 Cell number array is printed. Use wuprsst $=0$.

2 Cell number and surface number arrays are printed. Use LKRRNT=0.

FroRce Number of force structures. (0) When sForas $>0$, both the Force Structures section of NAMrIIST/DATA/ and the FORCE STRUCTURE SPECIFICATION CARDS are required.

IsTroc 0 No thermal structures are used. $(*)$ Do not include Thermal Structure input, or Thermal Structure Location Records in the input.

1 Thermal structures are used. NAMELIST $/ \mathrm{F} / \mathrm{.} / \mathrm{M} /$, and $/ \mathrm{T} /$, and Thermal Structure Location Cards are required in the input.

IsrBuc 0 The storage layout table is not printed. (*)

1 The storage layout table is printed.

-1 The input file listing is supressed.

IBSBDE 0 The BOUNDARY SURFACE sUMMARY is not printed. (")

1 The BOUNDARY SURFACE SUMMARY is printed, after which execution continues. For a description of the BOUNDARY SURFACE SUMMARY see the section entitled FINDING HOLES IN THE BOUNDARY in the appendix.

2 The BO'JNDARY SURFACE SUMMARY is printed, after which execution terminates.

IrIXIR 0 No subroutine timing information is shown (*)

1 Subroutine timing information summary.

2 Subroutine timing information is show after every call.

ITOBE $\quad$ By-pass the condenser calculation.

- 1 Condenser calculation via Option A. 
1 Condenser calculation via option B. (*)

The following variables are related to the condenser calculation.

IsEsil The index number in $x$-, $y$ - or $z$ - direction for separating the shell side and tube side geometrical location. It is used only when ITUBE (10)

ISEIAR 0 Vapor shear model is not used.

1 Berman's vapor shear model is used. (*)

2 Berman and Tumanov's vapor shear model is used.

IsUBC 0 Subcooled model is not used.

1 Subcooled model by Rohsenow is used. (")

2 Subcooled model by Chen is used.

INSODA 0 Innudation is not considered.

1 Innudation is considered. (")

IFOOL 0 Biofouling is not considered.

1 Biofouling is considered. (*)

\section{A.4.2.1 Restart Option}

There are two ways to force the code to write a restart file. The first is to allow the job to "max time". This is done by specifying large values for smanx and rIme. The amount of time remaining for the job is checked at the end of each iteration using the Argonne system routine TrEFT.

(See the appendix section entitled MACHING DEPENDENT ROUTINES.) If the amount of time remaining is greater than TREST, an input parameter in NAMELIST/DATA/, another iteration is performed. If not, a restart file is written.

The second way to obtain a restart file is to set wmax or mIxax to a time step or time which will be reached before the CPU job time expires. A restart file will be written at this time step or time. After a restart file is written, execution terminates.

When restarting from a previous run make sure that Ismare is set to the appropriate value. Also, it is advisable to delete all input for variables that one does not intend to change. In some cases variables will be reset back to their initial values if the input specification remains in the input stream. In short, the minimum input necessary is the correct input for restart cases.

IFRss 0 New case with no restart krritten. (*)

1 New case with restart written to unit 10.

2 Restart of previous run read from unit 9 with no restart written.

3 Restart of previous run read from unit 9 with restart written to unit 10.

\section{A.4.3 Boundary Surface Identification Records}

These records must be present only at the start of stead-state runs (ISTAT: $=0$ ). The purpose of this section is to specify a set of boundary surfaces which completely enclose the computational region and to define any other boundary surfaces inside the computational region. These interior boundary surfaces must completely surround a surface, a cell, or a 
group of cells. To completely surround a surface one must specify two boundary surfaces with normals in opposite directions. A single sided boundary surface is not allowed in the interior of the calculational region. Also be sure that all surfaces specified bound calculational cells. Each boundary surface is defined by specifying one or more BOUNDARY SURFACE IDENTIFICATION CARDS, each of which contains the following variables in FORMAT (A4,F 10.3.714):

NAME AREA IB IE JB JE KB KE N

NAME

REG The surface is a regular surface.

Regular surfaces lie on grid planes.

IREG The surface is an irregular surface.

Irregular surfaces do not lie on grid planes.

AREA REGULAR SURFACES

$<0.0$ The area of each surface element of cell $(1, \mathrm{~J}, \mathrm{k})$ is set to the product of its actual geometrical value, either $d x(1)^{*} d y(j)$, $d y(j) * d z(k)$, or $d x(i)^{*} d z(k)$, whichever is appropriate and absolute value of AREA.

$>0.0$ The area of each surface element of cell $(1, j, k)$ is set to AREA.

IRREGULAR SURFACE

$<0.0$ The absolute value is the slant length. The area of each surface element is set to slant length $D E L T$, where $D E L T$ corresponds to $D X, D Y$, or $D Z$ and is determined by the respective zero normal vector component.

$>0.0$ The area of each surface element of cell $(i, j, k)$ is set to AREA.

$\mathbf{I B}, \mathbf{I z}$ $\mathbf{J B}, \mathrm{J}$ $\mathbf{K B}, \mathbf{K} \mathbf{2}$

$\mathbf{N}$
These six variables are the beginning and ending $\mathrm{I}-, \mathrm{J}-$, and $\mathrm{K}$-indices that define a rectangular solid composed of one or more cells. The rectangular solid that defines or partially defines a surface is the one adjacent to and on the side pointed to by the surface normal. (Keep in mind that the surface

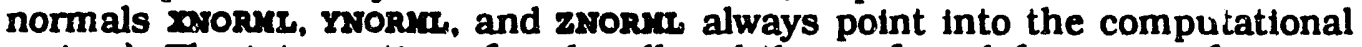
region.) The intersection of each cell and the surface defines a surface element.

The surface number. All surfaces with the same combination of the following three characteristics can be assigned the same surface number:

1. Velocity boundary condition.

2. Temperature boundary condition.

3. Unit normal vector to the surface.

Note 1. It is possible for two surface elements to lie in the same surface and have either the same or different surface numbers as well as for two surface elements to lie in different surfaces and have the same or different surface numbers.

Note 2. The order of the BOUNDARY SURFACE IDENTIFICATION RECORDS must be as follows:

1. All IREG records (irregular surfaces) must precede all REG records (regular surfaces).

2. The surface numbers, N, of all IREG records and REG records must be in the order of increasing value.

Note 3. When using cylindrical geometry (IGzox=-1), a surface must be specified at the origin when computational cells are bounded by the origin. When an annular region is teing modeled, a surface should not be cefined at the origin but rather at the boundary of the first (counting from the center) computational cell. Set XFLOW(N) $=-3$ and $\mathrm{x}$ swuP $(\mathrm{B})=400$ for surfaces defined at the origin.

Note 4. When using cylindrical geometry (I0zox=-1), with 2.0*PI radian geometries, $\mathrm{J}=1$ and $\mathrm{J}=\mathrm{JMAX}$ are automatically linked, thus, no 
surfaces need be defined at $Y=0.0$ and $Y=2.0 * P I$. the BOUNDARY VALUE INITIALIZATION CARDS.

\section{A.4.4 NAMELISTIDATA/}

ALPBA 0.0 Semt-implicit time advancement for both momentum and energy equations. 1.0 Fully-implicit time advancement for both momentum and energy equations. (*)

I rxox 0 No momentum calculation.

1 Momentum calculation is performed. (*)

IFINER 0 No energy calculation.

1 Energy calculation is performed. (")

IFMAss 0 No component mass fraction calculation (*)

1 Component mass fraction calculation is performed.

2 Component mass equations solved with mass diffusion.

ISKEW

Two finite differencing options for energy equation are available.

0 Pure Upwind Differencing scheme. (*)

2 Flow Modulated Skew Upwind Differencing scheme. This option reduces numerical diffusion inaccuracies present in the Pure Upwind Differencing scheme when the predominate flow is not orthogonal to the computational mesh.

The two variables below give the user some control over the frequency that the momentum and energy calculations are performed. The need for this control might arise in cases when one of the two fields (velocity or energy) varies slowly compared to the other. The intent is to be able to perform one of the calculations (momentum or energy) every time step while performing the other only occasionally resulting in a savings of CPU time. Before the user activates these variables it is highly recommended that $s /$ he understand the full implications of this approximation. The following combinations are allowed:

ISETMO $=1$ and ISETEN $=\mathrm{N}$ where $\mathrm{N}$ is any nonzero integer.

ISETHO $=\mathrm{N}$ and ISETER $=1$ where $\mathrm{N}$ is any nonzero in ieger.

ISITKO=M and ISETEN=N where one of the following conditions is satisfied: (1) $M<0$ and $N$ divides $M$ or (2) $N<0$ and $M$ divides $N$.

ISATES $\mathbf{N}$ When $\mathbf{N}$ is less than zero the energy calculation is turned off ever $-\mathrm{Nth}$ timestep.

$\mathbf{N}$ When $\mathbf{N}$ is greater than zero the energy calculation is turned on only every Nth timestep. (1)

I szTxo $\mathbf{N}$ When $\mathbf{N}$ is less than zero the momentum calculation is turned off every - $\mathrm{Nth}$ timestep.

$\mathbf{N}$ When $\mathbf{N}$ is greater than zero the momentum calculation is turned on only every Nth timestep. (1)

I STATR 0 Start of steady-state run. Geometry, boundary conditions, and initial conditions are specified from the input stream. Other parameters take default values or zero. (*)

1 Continuation of a steady-state run. Initial conditions are read from the restart file of a previous run in which steady-state has not yet been achieved. Some parameters may be changed in the input stream. 
2 Beginning of a transient run. Initial conditions are read from the restart file of a previous run. It is desirable that this previous run has achieved steadystate although not necessary. Some parameters may be changed in the input stream.

3 Continuation of a transient run. Initial conditions are read from the restart file of a previous beginning-of-transient run or continuation-of-transient run. Limited changes may be made in the input stream.

ITIBVC 0 No convergence information is printed.

1 Convergence information is printed every iteration.

The defaults for the following three values is 1 when $I \sin 25=0$, and 0 when ISTATY $=2$. In other cases these variables are ignored.

yEwrs 0 No new thermal structure input is read.

1 New thermal structure information is read if ISTROC $=1$ and ISTATE $=0$ or $I S T A T I=2$.

WEwror 0 No new force structure information is read.

1 New force structure information is read if sFORCs $>0$ and ISTATz=0 or IsTATr $=2$.

\section{A.4.4.1 Dobugging Paramotors}

Several debugging flags exist in the code which are intended primarily for use in the developmental stages. One generally needs the source listing in order to determine exactly what values are printed and under what conditions. The default value of all debugging flags is zero which indicates that no debugging printout is activated.

IDLBDG

IENBOG

IKABUC

ITRBVO

ITLBVO

IXXBVO

IYMBVE

IZMBVO

\section{A.4.4.2 Time and Time Stop Rolated Parameters}

DT (1) Time step size for time steps 1 through wastor, s. (0.1). This value is used oniy if IDTIXI:=0.

DT(2) Time step size for time steps after wasmor, s. (0.1). This value is used only if IDTIX $\boldsymbol{s}=0$.

IDrIMI 0 The tinie step size is taken from the user spectfied variable Dr. 
1 The time step size is computed internally as the product of the largest allowable time increment given the conditions (Courant time step size) and a user specified variable, RDTIME. (")

LASTDT

This variable in combination with $\mathrm{Dr}$ allows the user to change the time step size during a run. The time step size for all time steps through LAsTDT is taken from Dr(1). After step number LasrDr, the time step size is taken from $D r(2)$. (99999) This value is used only if IDTIKR=0.

NTBCON Up to ten values to specify the time step numbers to call SUBROUTINE GDCONV to calculate convergence criteria and the allowable time step size. The following are acceptable values of NrHCOts:

0 No further calls to ODCOsv.

$>0$ Time step number for which odcosv is called. After the Nth positive time step number in wracos has been processed, the $\mathrm{N}+1$ th value of NTBCON is used to determine subsequent calls to oDcowv.

$<0$ A value of $-\mathrm{N}$ indicates that ODCOsv is to be called every $\mathrm{Nth}$ time step. No subsequent values of NTHCON are considered. $(-1)$ See NTPRNT and NTPLOT for examples.

Nryax The maximum time step number for this run. Normal termination occurs after completion of this time step. (99999)

RDTIMB The time step size is computed internally as the product of the largest allowable time increment given the conditions and the variable, RDTIMr. (0.8) This value is used only if $I D T I M z=1$.

TIMAX The maximum time of this run. Normal termination occurs after this time has been reached. $s .(3.6 E+7)$ rIwax refers to the simulation or problem time and not the computer CPU time needed to run the problem.

TRIST The amount of time remaining for the job is checked at the end of each iteration. If the amount of time remaining is greater than TRssT another iteration is performed. If not, the restart file is written. When running long jobs or jobs requiring several seconds per iteration, one might wish to choose a larger more conservative value of TREST, s. (20.0)

This implementation depends on the Argonne system routine TLEFT which returns the time left until the total job time as specified on the JOB card has elapsed.

Note. In Sun work station. COMMIX-PPC calls the SUN FORTRAN Library Routine DrIxe to obtain the elapsed frun time in seconds for the calling process. When at the end of an iteration this elapsed time exceeds TRIsT, the calling process is terminated after optionally writing restart file. (3600.0)

TSTART Initial time, s. (0.0) This value should be reset to zero at the beginning of a transient run, ISTATz $=2$.

\section{A.4.4.3 Iteration Control Parameters}

The general definitions and default values of control parameters are given in this section. For a diagram showing the loop to which each variable relates, see the CONTROL PARAMETERS AT A GLANCE section in the appendix. 


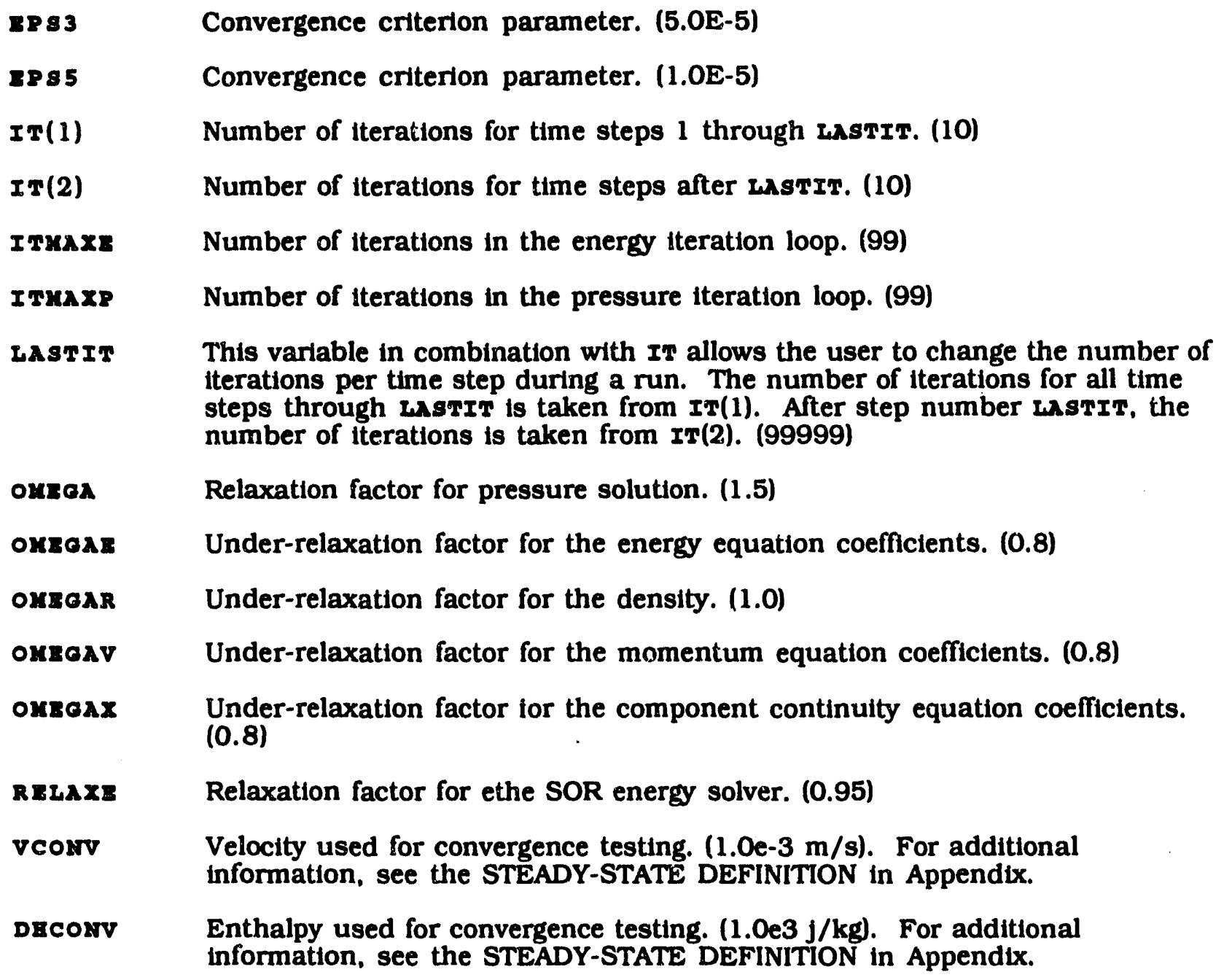

\section{A.4.4.4 Boundary Condition Types}

All external surfaces must have a velocity boundary condition type and a temperature or heat flux boundary condition type. Internal surfaces may also be assigned boundary condition types.

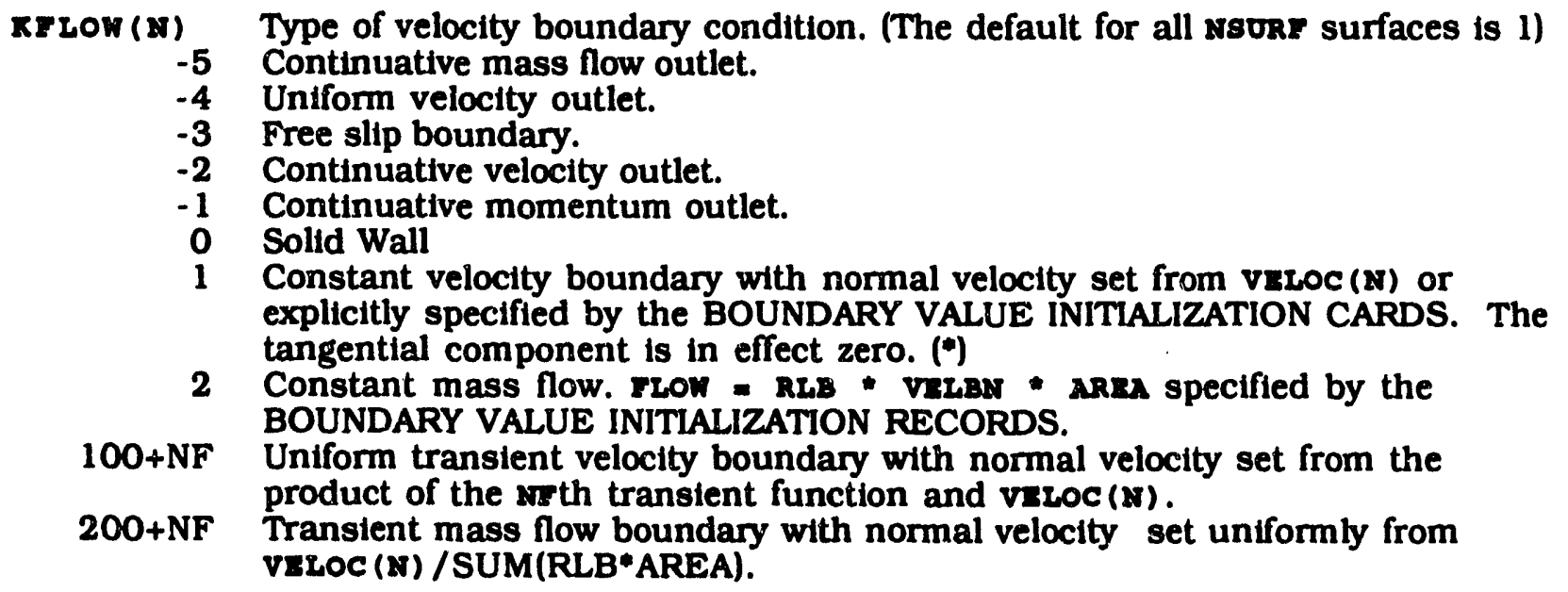


RPRIs (\$) Type of pressure boundary condition. Pressure boundary conditions are applied to the cells adjacent and interior to the boundary surface specified. (The default for all savar surfaces is 0 )

0 No pressure boundary condition is applied. (*)

1 Uniform constant pressure boundary with pressure set from PREs(N).

$100+\mathrm{NF}$ Uniform transient pressure boundary with pressure set from the product of the NFth transient function and PREs(N).

XTIXP (\&) Type of temperature or heat flux boundary condition. (The default for all nsore surfaces is 1)

1 Specified constant temperature boundary with temperature set from TIMP (M) or the BOUNDARY VALUE INITIALIZATION CARDS. (*) The surface heat flux is nominally computed considering the fluid conduction but not the presence of a wall. If one wishes to account for both the fluid convection and a wall conduction, the following four variables from the Wall Model section below must be specified: IBTWAL(N), WALLDX(N), and KATHAL (A) .

$100+\mathrm{NF}$ Uniform transient temperature boundary with temperature set from the product of the NFth transient function and TEMP(N). The surface heat flux is computed with the options as specified above for $\operatorname{KTEMP}(N)=1$.

200 Specified constant heat flux boundary with normal heat flux set from TEMP (N) or the BOUNDARY VALUE INITIALIZATION CARDS.

$300+N F$ Uniform transient heat flux boundary with normal heat flux set from the product of the NFth transient function and TRMP(N).

400 Adiabatic or zero diffusive heat flux boundary.

xuAss (N) Type of mass fraction boundary condition. Each value of xuss has an integer value of the form 'TSSCFF' which is coded according to the following rules:

T 0 Continuative mass fraction outlet.

1 Fixed specified mass frction.

2 Fixed specified volume fraction.

3 Transient mass fraction.

4 Transient volume fraction.

SS Surface number.

\section{A.4.4.5 Wall Model}

When specifying either a constant temperature boundary condition or a uniform transient temperature boundary condition $(\operatorname{KrExP}(\mathrm{N})=1$ or $\operatorname{RTRKP}(\mathrm{N})=100+\mathrm{NF})$ a wall may be modeled by defining the following three variables.

WaLLD(N) Wall thickness, m. (1.0)

MAтwaL (*) Material type for surface $N$. The value of this variable is used as the index NM in the Material Properties section below. (1)

Iнтพגน (*) Heat-transfer coefficient number for the calculation of heat-transfer between coolant and wall. The value of this vartable is used as the index NH in the Heat Transfer coefficient structure. (0)

Note. If the default value is taken, then the coolant to wall heat-transfer coefficient, if used. is evaluated simply as the fluid conductivity divided by the fluid conduction length. 


\section{A.4.4.6 Uniform Boundary Initialization}

The following three variables allow easy specification of uniform velocity, temperature or heat flux, and pressure values at boundaries. Nonuniform distributions can be specified by using the SURFACE ELEMENT INITIALIZATION RECORDS.

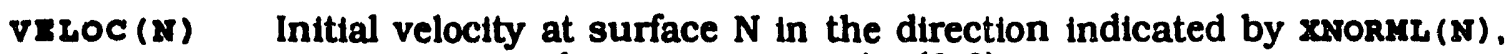

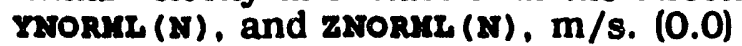

Trux (x) Initial temperature for surface N, C. (0.0). For a constant or transient heat flux boundary, race (N) contains the heat flux, $\mathrm{W} / \mathrm{m}^{* * 2} 2 .(0.0)$

PREs (N) Initial pressure fol surface $\mathrm{N}, \mathrm{Pa} .(0.0)$

\section{A.4.4.7 Uniform Coll Initialization}

The following variables allow easy specification of uniform cell temperature and pressure. Nonuniform distributions can be specified by using the CELL INITIALIZATION RECORDS.

TEKPO Initial temperature of all internal cells, C. (0.0)

PREs0 Initial pressure at the pressure reference point located at (xpRzso, yPRzso, zPRzs0). Pa. (1.01353E+5). The default pressure initializaion computes the static head using the density based on component 1 properties evaluated at rmapo, PRIso. The inital static head pressure at any point is computed with respect to the pressure reference point.

XPRESO X-coordinate of the pressure reference point, $m .(0.0)$

YPRESO Y-coordinate of the pressure reference point, $m .(0.0)$

ZRRESO Z-coordinate of the pressure reference point, m. (0.0)

GRAvX X-component of gravity vector, $\mathrm{m} / \mathrm{s} * \mathbf{2},(0.0)$

GRAVY Y-component of gravity vector, $\mathrm{m} / \mathrm{s} * 2$. (0.0)

GRAvz Z-component of gravity vector, $\mathrm{m} / \mathrm{s}^{* * 2}$. (0.0)

xxass (x) Initial mass fraction for surface $N$.

\section{A.4.4.8 Fluid-Structure Heat Transfer}

Fluid-Structure heat transfer (q) is computed as follows:

$$
q=A * h *(T s-T)
$$

where

A is the area,

$h$ is the heat transfer coefficient.

Ts is the temperature of structure, and 
Tf is the temperature of the fluid.

Heat transfer coefficient index, $\mathrm{kg}$, which is specified by the input variables IBTwar (w) in the WALL Model section and IET in the Thermal Structure Fluid Namelist/F/, is defined by three input parameters:

IBTCOR (AB) Heat transfer coefficient correlation number.

atcrex (MR) Characteristic length.

ETCXOL (NB) heat transfer coefficient multiplier.

When the correlation number, InrcoR (18R), is in the range (1-10), then the heat transfer coefficient, $h$, is defined as follows:

$$
h=\operatorname{ATCKOL}(\mathrm{NB}) * \mathrm{Nu} * \mathrm{k} / \operatorname{BTCLEN}(\mathrm{NB})
$$

where

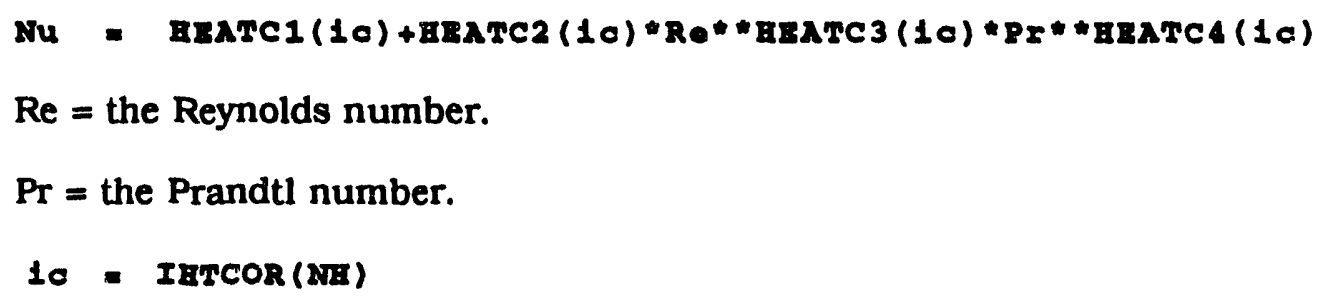

grarci $(10)$ is Nusselt number coefficient.

Since the Nusselt number, Nu, must always be positive, matc1(2R) should be positive to accomodate a zero flow situation. (5.0)

JEATC2 (10) Nusselt number coefficient. (4.02E-4)

geatc3 (1c) Nusselt number coefficient. (0.8)

mEATcs (1c) Nusselt number coefficient. (0.0), and

$\mathbf{k}$ is the fluid conductivity.

When the correlation number, Imscos(sra), is in the range (11-20), then the heat transfer coefficient, $h$, is defined as follows:

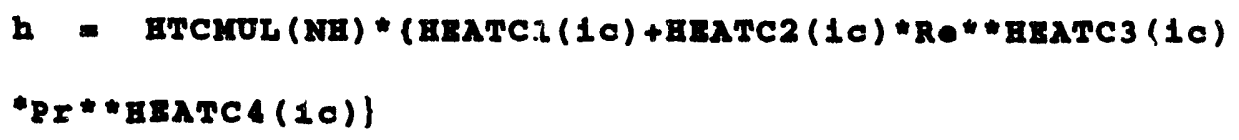

where

$$
\text { tC=IATCOR (NR) }-10 \text {. }
$$

When Heat transfer correlation number, IHTCOR(NR), is in the range (21-50), then it is referred to the condenser shell-side and tube-side heat transfer correlation. Detalls may be described as follows. 
1. InTCOR (NE) =21-30 For shell-side heat transfer correlations. When InTCOR(NR) $=21$, correlations by Nusselt's condensation on horizontal tubes and the effect due to non-condensable gas by Berman and Fuks are used.

2. $\operatorname{InTCOR}(\mathrm{sIE})=31-40$ For tube-side heat transfer correlations. The heat transfer coefficient, $h$, is calculated according to equation (A) with $10=\operatorname{InTCOR}(\mathrm{NBE})-30$.

When blofouling in inner tube wall is considered, the following varlable must be specified in NAMELIST/DATA/.

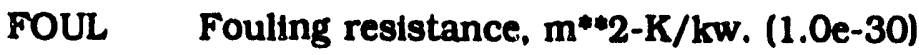

\section{A.4.4.9 Matorial Proportios}

Material Properties are needed when modeling the fluid in the computational cells (MATYPE(x)) and optionally walls (marwar(x).). The values of marypz(x) and matwax (N) may be any integer from 1 to 22. When the value is in the range of 1 to 20 , the material properties are computed from the fast running simplified straight-line approximations to the state equations. When the value is in the range of 21 to 22 , the material propertites are computed from the rigorous equation-of-state subroutines.

MaTrPE (K) Simplified material properties for compoment or material $\mathrm{K}$ (For value from 1 to 20 ).

Conductivity in $\mathrm{W} /(\mathrm{m}-\mathrm{C})$ :

$\mathrm{COND}=\mathrm{COK}(\mathrm{MA})+\mathrm{C} 1 \mathrm{~K}(\mathrm{MA}) * \mathrm{TC}+\mathrm{C} 2 \mathrm{~K}(\mathrm{MA}) * \mathrm{TC} * 2$

Density in $\mathrm{kg} / \mathrm{m} * \mathrm{~m}^{\mathrm{m}}$

DENSE $=C O R O(M A)+C 1 R O(M A) * T C+C 2 R O(M A) * P A / T K$

Enthalpy in J/kg:

$\mathrm{ENTH}=\mathrm{COH}(\mathrm{MA})+\mathrm{C} 1 \mathrm{H}(\mathrm{MA}) * \mathrm{TC}+\mathrm{C} 2 \mathrm{H}(\mathrm{MA}) * \mathrm{TC} * 2+\mathrm{C} 3 \mathrm{H}(\mathrm{MA}) * \mathrm{PA}$

Saturation pressure in $\mathrm{Pa}$ :

PSAT $=E X P(C O P(M A)+T C * C 1 P(M A)+T C * T C * C 2 P(M A))$

Viscosity in Pa*s:

VISC $=\operatorname{COMU}(M A)+C 1 M U(M A) * T C+C 2 M U(M A) / T K$

Molecular weight of material :

MOLWT $=$ WTMOL(MA)

Binary molecular diffustvity $[\mathrm{m} * \mathrm{~m} / \mathrm{s}]$ :

DIFFIM $=(C O D(M A)+C 1 D(M A) / P A) * T K * " C 2 D(M A)$ 
Note. When COD(MA) $<0.0$ and $C 1 D(M A)<0.0$. DIFFIM is evaluated from the formula developed by Slattery and Bird.

where

$T C$ is the temperature in degrees $C$.

TK is the temperature in degrees $K$.

PA is the pressure in Pascals, and

$H$ is the enthalpy in $\mathrm{J} / \mathrm{kg}$.

DIFuסd The multiplier for the binary molecular diffusivity.

MTYYP (x) CODED VALUE OF MATERIAL TYPE FOR COMPONENT K. (For value from 21 to 22)

The following values of EATYPI are associated with rigorous equation-of-state subroutines. This list may be expanded with little difficulty by users having more accurate equation-of-state subroutines for their materials.

21 Water liquid

22 Water vapor

Note: In condenser application, marye (1) and marys (2) are reserved for steam and air respectively.

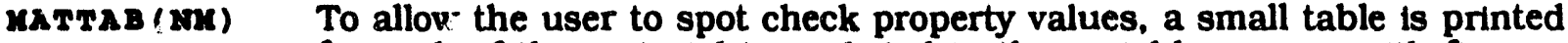
TABLOT (MM) for each of the material types listed in the variable vaTrAB with five TABEIT (MM) temperature values ranging from the corresponding entries in rABLOT to TABHIT at a pressure of PRIs0. For example, one could obtain a table of property values computed from the coefficients $\operatorname{COK}(1), \mathrm{C} 1 \mathrm{~K}(1), \mathrm{C} 2 \mathrm{~K}(1)$. CORO(1), C1RO(1), C2RO(1), $\mathrm{COH}(1), \ldots$ etc., over a temperature range from 500.0 to 900.0 degrees $\mathrm{C}$ and a table of water liquid property values over the range from 20.0 to 90.0 degrees $\mathrm{C}$ by including the following input in NAMELIST/DATA/:

MATTAB $=1.21$, TABLOT $=500.0,20.0$, TABRIT $=900.0,90.0$,

The default values for the above three variables are zero thus no property tables are printed unless these values are specified.

\section{A.4.4.10 Transiont Functions}

All transient drtving functions are input into the following three variables. They must be input at the beginning of the transient (Isтxis=2) even if they have been input previously. Each function is defined by a user specified set of points. Cubic spline fit coefficients are then generated in SUBROUTINE FITIT. Fifty equaliy spaced values are printed to allow the user to check the adequacy of the input distribution. Ten to fifteen values with points concentrated at rapidly changing $Y$-values should be adequate. Currently the total number of points allowed for the specification of transient functions is one hund $-d$.

TVAL (MP) The independent variable, usually time, for the transient functions. 
rVaI (NP) The dependent variable for the transient functions. The first value of the second function immediately follows the last value of the first function. The same pattern must be followed for all subsequent functions. Make sure that the entire range of the function used lies within the range input as the fitting routine does not extrapolate. Discontinuities are indicated by specifying the same X-coordinate twice with the same or different Y-coordinate values.

IIND (AF) The number of points in the. NFth transient function

wrors In order to simplify thermal structure input in certain cases, the heat source transient function numbers can be overridden in NAMELIST/DATA/. These values are input into the variable swors in the order in which the thermal structure prototypes were defined. Any values specified in swors will override all other input and previous values. If no values of wrors are defined, no changes to the heat source transient function numbers are made.

Norer Number of the transient function which is used as a multiplier of the heat source for the coolant when thermal structures are present and as a multiplier of total heat source when no thermal structures are present. (0)

\section{A.4.4.11 Plot Tape Option}

IrPLOT Up to 25 values to specify when plotting information is to be written to unit 76. The following are acceptable values of NTPLOT:

0 No more plotting information is written to unit 76. (*)

$>0$ Time step number for which plotting information is written to unit 76. After the Nth positive time step in NTPLOT has been processed, the $\mathrm{N}+1$ th value of NTPLOT is used to determine subsequent writes to the plot file.

$<0 \quad A$ value $-N$ indicates that information is written to unit 76 every $N$ th time step. No subsequent values of ETPLOT are considered.

Example. NrPLOT $=-5$ indicates that every 5 th step is to be processed. ITPLOT $=5,10,-20$ indicates that steps $5,10,20,40,60$, etc., are to be processed. MrPLOT $=10,20,0$ indicates that only steps 10 and 20 are to be processed.

\section{A.4.4.12 Printing Option}

Calls to SUBROUTINE OUTPUT are controlled by the two variables, WTPRIT and TPRur. They can be used individually or together. The information printed at each call to SUBROUTINE OUTPUT is determined by the variables ISTPR and NTBPR described below.

NTPRAT Up to 50 time step numbers at which SUBROUTINE OUTPUT is to be called. The following are acceptable values for MTPRNT:

0 No more calls to SUBROUTINE OUTPUT. When restarting, previous specification of ATPRar values may be overridden by specifying the desired new values followed by a zero in MTPRuTr.

$>0$ Time step number for which SUBROUTINE OUTPUT is to be called. After the Nth positive time step in MrPRarr has been processed the $\mathrm{N}+1$ th value of NTPRNT is used to determine subsequent calls to OOTPOT.

$<0 \quad A$ value $-\mathrm{N}$ indicates that SUBROUTINE OUTPUT is called every $\mathrm{N}$ th time step. No subsequent values of STPRArs are considered. -9999..SUBROUTINE oorpor is called just before the run is terminated. (*)

Example. NTPRNT $=0$ indicates that after initialization, SUBROUTINE OUTPUT is never called. ArPRMr $=5,10,-9999$ indicates that SUBROUTINE OUTPUT is called at steps 5, 10, and just before termination. 
TPRNT

Up to 50 times (problem time in seconds) at which SUBROUTINE OUTPUT is to be called. The following are acceptable values of TPRNT:

0.0 No more call to SUBROUTINE OUTPUT. (") When restarting, previous specification of TPRars values may be overridden by specifying the desired new values followed by a zero in TPRsr.

>0.0 Times at or after which SUBROUTINE OUTPUT is to be called. When or after the Nth positive time in TPRs. has been processed, the $\mathrm{N}+1$ th value of TPrury is used to determine subsequent calls to oorpor.

$<0.0$ A value of -T indicates that SUBROUTINE OUTPUT is to be called at $T$ second intervals. If the $\mathrm{Nth}$ value is negative, then the $\mathrm{N}+1$ th value stores the next time value at which oorsor is to be called. This is nominally set to zero but can be specified by the user. No subsequent values of mPRST are considered.

Example. TPRNT =1.0,5.0,-10.0 indicates that OOTPOT is to be called at or after times 1.0, 5.0, 10.0, 20.0, .. etc.. TPRSTr $=-5.0,10.0$ indicates that oorpors is to be calleu at times $10.0,15.0 .20 .0 . .$. . etc..

ISTPR Up to Afty coded values which specify the arrays to be printed in the first call to SUBROUTINE OUTPUT. (0)

NTEPR Up to fifty coded values which specify the arrays to be printed in. all calls after the first call to oOrPOr. For internal arrays, each value of ISTPR and IFrAPR is a signed seven digit integer of the form 'SCVVPLLL' which is coded according to the following rules:

C Fluld component or phase number. (1)

S + Only the plane specified by 'PLLL' is printed. (*) A plus sign is assumed and need not be specified.

- All planes between the values of 'LLL' on the current and following values of IgTPR or MTraPR are printed.

VV 01 PsTato U-component of velocity. $[\mathrm{m} / \mathrm{s}]$.

02 VI V-component of velocity. $[\mathrm{m} / \mathrm{s}]$.

03 WI W-component of velocity. $[\mathrm{m} / \mathrm{s}]$.

$04 \mathrm{EI}$ Enthalpy. $(\mathrm{J} / \mathrm{kg}$.

05 TI Temperature, [deg. Cl.

06 is Volume porosity.

07 RI Density. $[\mathrm{kg} / \mathrm{s}]$.

08 P Static Pressure, [Pa].

09 DI Residual mass, [kg].

10 ALX X-direction surface permeability.

11 AIY Y-direction surface permeability.

12 ALZ Z-direction surface permeability.

13 RLOLD Density at previous time step. [kg/m*3].

$14 \mathrm{TK}$ Turbulent kinetic energy. $[\mathrm{J} / \mathrm{kg}]$.

15 Qsoor Volumetric heat source, [W/m*3].

16 -POID Pressure change from last time step, [Pa].

17 P-sstaro Pressure minus static pressure.(Pa).

18 TEL VOLUME fraction.

19 P-PREso Pressure minus inittal pressure,[Pa].

20 Torcos Turbulent conductivity. $[\mathrm{W} / \mathrm{m} / \mathrm{C}]$.

21 TORVIS

22 TELOLD

23 TIOLD

24 VIOLD

25 WLOLD

26 EO.LD

27 POID

Trubulent viscosity. (Pa-s].

Volume fraction from previous time step. U-component of velocity at previous step. $[\mathrm{m} / \mathrm{s}]$.

$\mathrm{V}$-component of velocity at previous step. $[\mathrm{m} / \mathrm{s}]$.

W-component of velocity at previous step. [m/s].

Enthalpy at previous step. $[\mathrm{J} / \mathrm{kg}]$.

Static pressure at previous step.|Pa]. 


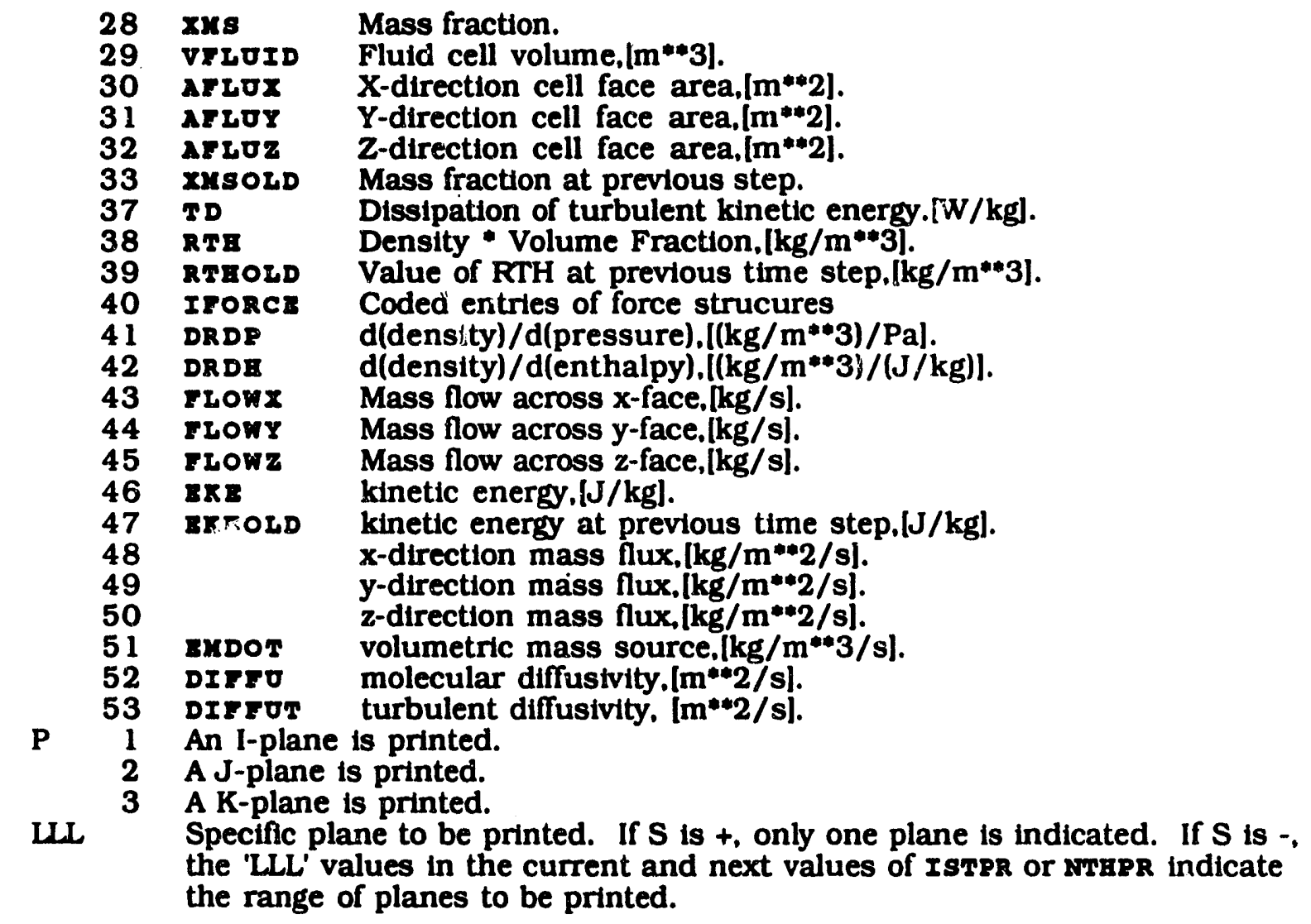

For thermal structure information, each value of ISTPR and IrraPR is a signed seven digit integer of the form 'SC8NNNNN' which is coded according to the following rules:

C Fluid component or phase number. Values of 0 thru 9 correspond to components 1 thru $10 .(0)$

$S+$ Only structure number 'NNNN' is printed. (*) A plus sign is assumed and need not be specified.

- All structure between the values of 'NNNN' in the current and following values of ISTPR and NTEPR are printed.

NNNNN Specific structure to be printed. If $S$ is ' + ', only one structure is indicated. If $S$ is '-', the 'NNNNN' values in the current and next values of ISTPR or STEPR indicate the range of surfaces to be printed.

For surface arrays, each value of ISTPR and srmpR is a signed seven digit integer of the form 'SC9VVLLL' which is coded according to the following rules:

C Fluid component or phase number. (1)

$S+$ Only the surface number 'LLL' is printed. (") A plus sign is assumed and need not be spectfied.

- All surfaces between the values of 'LLL' in the current and following values of ISTPR or WTEPR are printed.

VV 01 VBLBN Normal surface velocity. $[\mathrm{m} / \mathrm{s}]$.

02 QBA Normal surface heat flux.[W/m**2].

03 uB Adjacent internal cell number.

04 ELB Surface enthalpy. $(\mathrm{J} / \mathrm{kg}$.

05 TIB Surface temperature.ldeg. $\mathrm{Cl}$.

06 ARIA Surface element area, $(m+2)$.

07 RLB Surface density, [kg/m $/ \mathrm{m}^{+4}$ ].

08 P B Surface pressure, [Pa]. 


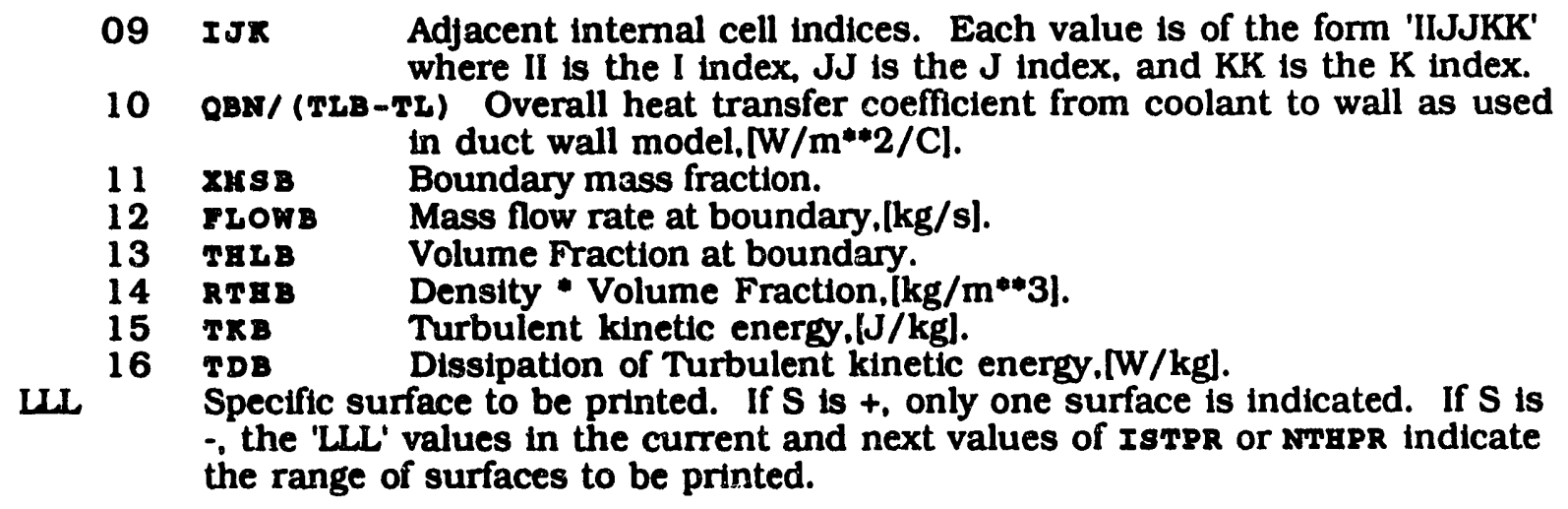

Example. ISTPR $=06105,-16301,-10305$, NTHPR $=01105,-02301,-$ $02305,90101,-90501,-90505$, indicates that the first call to oUTPOT will print the $I=5$ plane of volume porosity and $K$-planes 1 through 5 of the $X$ direction surface permeability. On all subsequent calls, to OUTPOT, the $I=5$ plane of the U component of velocity, K-planes 1 through 5 of the $V$ component of velocity, the boundary velocity for surface 1 , and surface temperature for surfaces 1 through 5 .

scor Tx The number of columns to be written in the tabular output. Valid values for weorox are 8, 9, 10, and 11 . The formats used are 1PE13.6, 1PE 11.4. 1PE10.3, and 1PE9.2 respectively. (10)

IJKRE A three digit binary number which determines the row-column orientation of tabular output. The following table gives the acceptable values. (101)

\begin{tabular}{|c|c|c|c|c|c|c|}
\hline & \multicolumn{2}{|c|}{ I-plane Indices } & \multicolumn{2}{|c|}{$\mathrm{J}$-plane Indices } & \multicolumn{2}{|c|}{$\mathrm{K}$-plane Indices } \\
\hline & Row & Column & Row & Column & Row & Column \\
\hline 000 & $\mathbf{J}$ & K & $\mathbf{K}$ & I & I & $\mathbf{J}$ \\
\hline 001 & 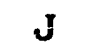 & $\mathbf{K}$ & $\mathbf{K}$ & I & $\mathbf{J}$ & I \\
\hline 010 & $J$ & $\mathbf{K}$ & I & $\mathbf{K}$ & I & $J$ \\
\hline 011 & $\mathbf{J}$ & $\mathbf{K}$ & I & $\mathbf{K}$ & $\mathbf{J}$ & I \\
\hline 100 & $\mathbf{K}$ & $\boldsymbol{J}$ & $\mathbf{K}$ & I & I & $\mathbf{J}$ \\
\hline 101 & $\mathbf{K}$ & $\mathbf{J}$ & $\mathbf{K}$ & & $J$ & I \\
\hline 110 & $\mathbf{K}$ & $\boldsymbol{J}$ & I & $\mathbf{K}$ & I & $\mathbf{J}$ \\
\hline 111 & $\mathbf{K}$ & $\boldsymbol{J}$ & I & $\mathbf{K}$ & $\mathbf{J}$ & I \\
\hline
\end{tabular}

\section{A.4.4.13 Force Structures}

The force structure is a mechanism whereby a drag or resistance force in $\mathrm{Pa} / \mathrm{m}$ can be applied to a fluid flow across a cell face between two calculational cells. The location of each force structure, NF, is specified in the FORCE STRUCTURE SPECIFICATION CARDS. The generic force structure applies a resistance of one of the following forms:

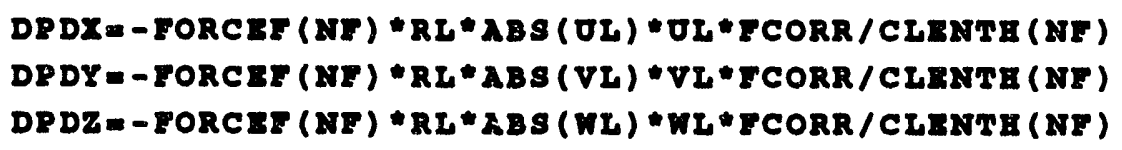

Where FCORR=ACORRL (NC) *RR* \#BCORRL (NC) +CCORRL (NC)

when RIl < REYTRN(NC),

and TCORR=ACORRT (RC) *RE**BCORRT (NC) +CCORRT (NC)

when RII >a REYTRN(NC),

and RIF RI*SQRT (UL**2+VL**2+WL**2)*REYIEN (NE)/VIS, 
and

$R L$ is the local density,

OL, VL, and $\mathrm{wL}$ are local velocities,

vIs is the local viscosity, and

we is the correlation index, ICORR(NF).

FORCE: (NF) Force coefficient for force structure sTr.

REYLIN(NF) Length used to compute the Reynolds number for force structure NF, $\mathrm{m}$.

CLENSa (MF) $>0.0$ The value input is used as the characteristic length in the above equation.

$<0.0$ A characteristic length computed from either DX, DY, or DZ, whichever is appropriate, is used for CLINPrE (NF) in the above equation.

ICORR (NF) The correlation index of force structure sF. For the generic force structures the values of ICORR must be less than 21 . These values are used as indices of the user specified correlation variables below.

Note. For the valve model IcorR is set by $100 *$ ( the valve \# NV ) + ICORR.

NCORR

The number of correlation types available for force structures. This value must equal or exceed the maximum value specified in ICORR but be less than 21 .

REYTRN(NC)

ACORRL (NC)

BCORRL (NC)

CCORRL (NC)

ACORRT (NC)

BCORRT (NC)

CCORRT (NC)
The transition Reynolds number. Correlation coefficients when the Reynolds number above, $R E$, is in the laminar regime.

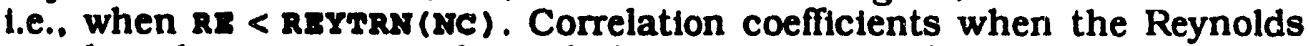
number above, $R E$, is in the turbulent regime,i.e., when $R B>=$ REYTRN (NC).

A pressure relief valve can be modeled by specifying a force structure with a correlation index of the form ' $V V C C$ ' where 'VV' is the valve number, $N V$, ranging from 1 to 19 , and 'CC' is the correlation index. Each valve, NV, has associated with it the following variables:

IVALVE( (aV)

The state of valve NV. A value of 1 indicates that the valve is open and a value of 0 indicates that the valve is closed. (0)

DPOPEN(AV) The pressure gradient between two cells at which a closed valve opens, Pa. (1.OE+20)

DPCLOS (MV) The pressure gradient between two cells at which an open valve closes. Pa. $(-1.0 E+20)$

Note. The pressure gradient between two cells is computed as $P(I, J, K)$ $P(I+i, J, K)$ for $x$-direction valves, $P(I, J, K)-P(I, J+1, K)$ for $y$-direction valves, and $P(I, J, K)-P(I, J, K+1)$ for z-direction valves.

When a valve is closed a very high resistance is applied across the cell face. When a valve is open a resistance can be applied in one of two ways. By specifying a correlation Index IC in the range of 1 through 20, a generic force structure as defined above can be applied. When choosing this ortion the user can specify RIYLEN(NF)=1.0, CLENTH(NF)= ALPEA, the local volume porosil.y, $\operatorname{REYTRN}(\mathrm{IC})=1.0 E+10, \operatorname{ACORRL}(I C)=1 / \mathrm{VIS}$, where VIS is the local viscosity, $\operatorname{sCORRL}(I C)=-1.0$, and $\operatorname{ccoRRL}(I C)=0.0$, the resistance is then given by -rORCEF (NF) "WL, where $W L$ is the local velocity. Alternatively, one can specify a force 
correlation of 70 and the valve velocity will be automatically computed according to the valve velocity model.

Several other specific structures can be modeled by including a force structure with a correlation index listed below.

$\begin{array}{lll}\text { ICORR (NF) } & 50 & \mathrm{dP} / \mathrm{dx} \text { proportional to velocity (U). } \\ 60 & \mathrm{dP} / \mathrm{dx} \text { proportional to mass flux (RO*U). } \\ 61 & \text { Specified mass flux. RO*U = FORCEF (NF). } \\ 90 & \text { CRBR fuel assembly } \\ 91 & \text { CRBR blanket assembly } \\ 92 & \text { DRHX (Direct Reactor Heat Exchanger) } \\ 93 & \text { CRBR chimney assembly } \\ 94 & \text { FFTF pin bundles } \\ 95 & \text { CRBR control assembly } \\ 96 & \text { Axial flow through vertical rod bundles. }\end{array}$

FORCBF (NF) Force coefficient multiplying the resistance computed for the specific structure.

REYLEN (NF) When modeling axial flow through vertical rods, correlation index 96, this value defines the length used to compute the Reynolds number.

A precise description of the resistance being modeled can be found in the source code for SUBROUTINE FORCES or in the doccument entitled "Some Resistance Correlations for COMMLX Users" by Sha and Shah, ATHRP-13, (April 1९)83).

\section{A.4.4.14 Turbulence Modeling}

In all of the following turbulence models an effective viscosity is used in the diffusion term of the momentum equation. This effective viscosity is the sum of the turbulent viscosity and the the molecular viscosity. Similarly an effective thermal conductivity is used in the diffusion term of the energy equation which is likewise the sum of the turbulent thermal conductivity and the molecular thermal conductivity.

\section{A.4.4.14.1 Constant Turbulont Diffusivity Model}

The turbulent viscosity and turbulent conductivity are assumed constant everywhere.

ITURRI 0 Turbulent kinetic energy flag must be zero. (*) The variable ITURKI must be input in NAMELIST/GEOM/.

TORBV Turbulent viscosity, Pa-s. (0.0) This must be set to some non-negative value.

TORBC Turbulent conductivity, W/(m-C). (0.0)

\section{A.4.4.14.2 Two-Equation Turbulence Model}

This is the most rigorous turbulence model for practical engineering problems. Both the equation for turbulent kinetic energy (TK) and the equation for dissipation of turbulent kinetic energy (TD) are solved. Wall function corrections are applied to cells adjacent to solid walls for both the turbulent kinetic energy equation and momentum equations. The turbulent viscosity is computed using the following equation: 


\section{TORVIS =CDTURB*RO*TK**2/TD}

where CDTORB is the coefficient for computation of shear stress near the wall, RO is the local density.

TX is the local turbulent kinetic energy, and

TD is the dissipation of turbulent kinetic energy.

Note: Values are initialized only when Istatz: $=0$ or ISTAтs $=2$.

For this option the following input must be specified:

ITORK: 12 Turbulent kinetic energy flag must be 12. The variable Irorks must be input in NAMELIST/GEOM/.

ARAP PA Wall constant. (0.42)

CDTORB Coefficient for computation of shear stress near the wall. (0.09)

Cr1 Empirical constant used in the equation to compute turbulent kinetic energy. (1.44)

CT2 Empirical constant used in the equation to compute the dissipation of turbulent kinetic energy. (1.92)

$\mathbf{E x}$

Wall constant. (9.0)

EPS 6

Convergence criterion parameter for turbulent kinetic energy equation. (1.0E-5)

IтxAxR Maximum number of iterations for turbulent kinetic energy equations. (29)

OMrexD Relaxation factor for equation to compute dissipation of turbulent kinetic energy. (0.7)

oxseax Relaxation factor for equation to compute the turbulent kinetic energy. (0.7)

OxroxT Relaxation factor for turbulent viscosity. (0.7)

PRNDID Turbulence Prandtl number for dissipation of turbulent kinetic energy. (1.3)

PRNDLE Turbulence Prandtl number for thermal energy transfer. (0.9)

PRNDIR Turbulence Prandtl number for turbulent kinetic energy (1.0)

RELAXX Relaxation factor for turbulent kinetic energy solution (0.8).

TDXIX The smallest value allowed for turbulence dissipation. All values of turbulence dissipation computed to be less than TDMIN will be set to TDMIN. (1.0e-10)

TXXIN The smallest value allowed for turbulence kinetic energy. All values of turbulence kinetic energy computed to be less than rkxis will be set to TXxIN. (1.0e-16) 


\section{A.4.5 Force Structure Specification Records}

These records must be included only when NFORCr $>0$ and NEwFOR $=1$ in NAMELIST /GEOM/. These records are used to locate the force structures described in the Force Structure section of NAMELIST/DATA/. These forces can be applied at cell faces between two computational cells. The locations therefore correspond to portion of grid planes. Each card in this section contains the following variables in the FORMAT (A4,7I4).

NAME N IB IE JB JE KB KE

NAME XFOR X-direction force.

YFOR Y-direction force.

ZFOR Z-direction force.

$\mathbf{N}$

Force structure number.

$I B, I B$

$\checkmark B, J \mathbf{B}$

These six variables are the beginning and ending $\mathrm{I}-, \mathrm{J}-$, and $\mathrm{K}$-indices used to define a plane of cells. The cell face defined by cell $(I, J, K)$ for an X-direction force is that one between cells $(I, J, K)$ and $(I+1, J, K)$. For a Y-direction force, it is the one between cells $(I, J, K)$ and $(I, J+1, K)$, and for a Z-direction force, it is the one between cells $(I, J, K)$ and $(I, J, K+1)$.

\section{A.4.6 Thormal Structure Records}

This set of records is included if and only if ISTROC $=1$ and NEWTS $=1$ in NAMELIST/GEOM/.

A thermal structure is a collection of thermal structure elements each of which has the same characteristics as specified by a thermal structure prototype. Thermal structure prototypes are defined using TYPE. FLOID, and kATERIAL namelists with the names T, F. and $M$ respectively. The order in which these namelists are input indicates the the construction of the thermal structures and must conform to the following rules:

1. A TYPE namelist must begin the definition of each thermal structure prototype.

2. If fluid interacts with surface one, a FLOID namelist must be present after the TYPR namelist (before the first mararal namelist). If, in addition, fluid interacts with surface two, a FLoID namelist must also be present after the last matrRIAL namelist.

3. A gap exists after each material except the last. The gap parameters are specified in the matreras namelist.

4. The initial default for all namelist variables is zero. Subsequent defaults are the values in effect after reading the previous namelist. If, for example, the geometrical type is the same for all thermal structure prototypes, IXYZ need be specified only on the first TYPE namelist.

5. The definition of thermal structure prototype $N+1$ must follow the definition of thermal structure prototype $\mathrm{N}$. 
6. Blank cards or cards with blanks in columns 1 through 4 may be interspersed as desired.

The precise definition of each card is as follows:

\section{A.4.6.1 TYPE NAMELIST/TI}

$\boldsymbol{N}$

IXYZ

sq

RODFR

$>0$ Number or fraction of actual rods interacting with each associated coolant cell.

$<0$ The absolute value is the number or fraction of rods per unit area $(m * * 2)$ interacting with each associated coolant cell.

Slab thermal structures:

$>0$ Slab area in each associated coolant cell, $m * 2$.

$<0$ The absolute value is the slab area divided by the cell area. In the case of two sided thermal structures this value is equivalent to a solid permeability for the structure.

Spherical thermal structures:

$>0$ Number or fraction of spheres interacting with each associated coolant cell.

$<0$ The absolute value is the number or fraction of spheres per unit volume $\left(m^{*} 3\right)$ interacting with each associated coolant cell.

OUTR structures.

\section{A.4.6.2 Fluid NAMELIST/F/}

I $\mathbf{B T}$

Heat transfer correlation index. This value is used as the index, sta, of the vartables mextc1, Bzarc2, Bzarc3, and grars described in the FluidStructure Heat Transfer section of NAMELIST/DATA/.

EY D

Hydraulic diameter or reference length. this value is used as $D$, the reference length, as described in the Fluid-Structure Heat Transfer Section of NAMELIST/DATA/.

\section{A.4.6.3 Matorial NAMELIST/M/}


NP

$\mathbf{D R}$
Number of partitions in the material. A thermal structure temperature will be computed for each material partition.

Partition size, m.

Volumetric heat source for the material region, $\mathrm{W} / \mathrm{m} * 3$.

The following gap properties must be correctly specified or defaulted only when another material follows. If a fluid follows, the gap properties are ignored.

SOAR

Gap słze, m. (0.0)

EGAP

Gap heat transfer coefficient, $W /\left(m^{* * 2}-C\right)$. (1.0)

\section{A.4.7 Thermal Structure Location Records}

This set of records is included if and only if ISTroc $=1$ and Nawrs $=1$ in NAMELIST /GEOM/.

Once the thermal structure prototypes have been defined the location of the thermal structure elements are specified by the THERMAL STRUCTURE LOCATION CARDS. These cards contain the following variables in FORMAT $(A 4,714)$

LOC NUM IB IE JB JE KB KE

IOC ODT The cells specified interact with the outside or surface 1 .

IN The cells specified interact with the inside or surface 2.

NOX Thermal structure prototype number.

IB, IE

$\mathbf{S B}, \mathbf{J a}$

These six variables are the beginning and ending $\mathrm{I}-. \mathrm{J}-$, and $\mathrm{K}$-indices that define a rectangular (cylindrical) solid composed of one or more cells which KB, XE. are to interact with thermal structure sox.

Note 1. A cell should not be specified twice by the indices unless the true intention is to have two occurrences of the thermal structure prototype sox.

Note 2. Many THERMAL STRUCTURE LOCATION CARDS may be needed to define all the cells interacting with a given thermal structure prototype.

Note 3. The order in which cells are specified is arbitrary except when the thermal structure prototype has fluid cells interacting with both surfaces. In this case cells are paired off in the order in which they are specified. The number of cells interacting with one surface must equal the number of cells interacting with the other surface.

\section{A.4.8 Coll Initialization Rocords}

The purpose of this set of records is to permit initialization of internal cell values of any of the array listed below. Uniform temperature can be more easily specified using the 
variable Twis in NAMELIST/DATA/. Each line in this section contain the following varlables in the FORMAT (A4,F10.3,914).

\author{
NAME RVAL IB IE JB JE KB KE N NCOM
}

NAME

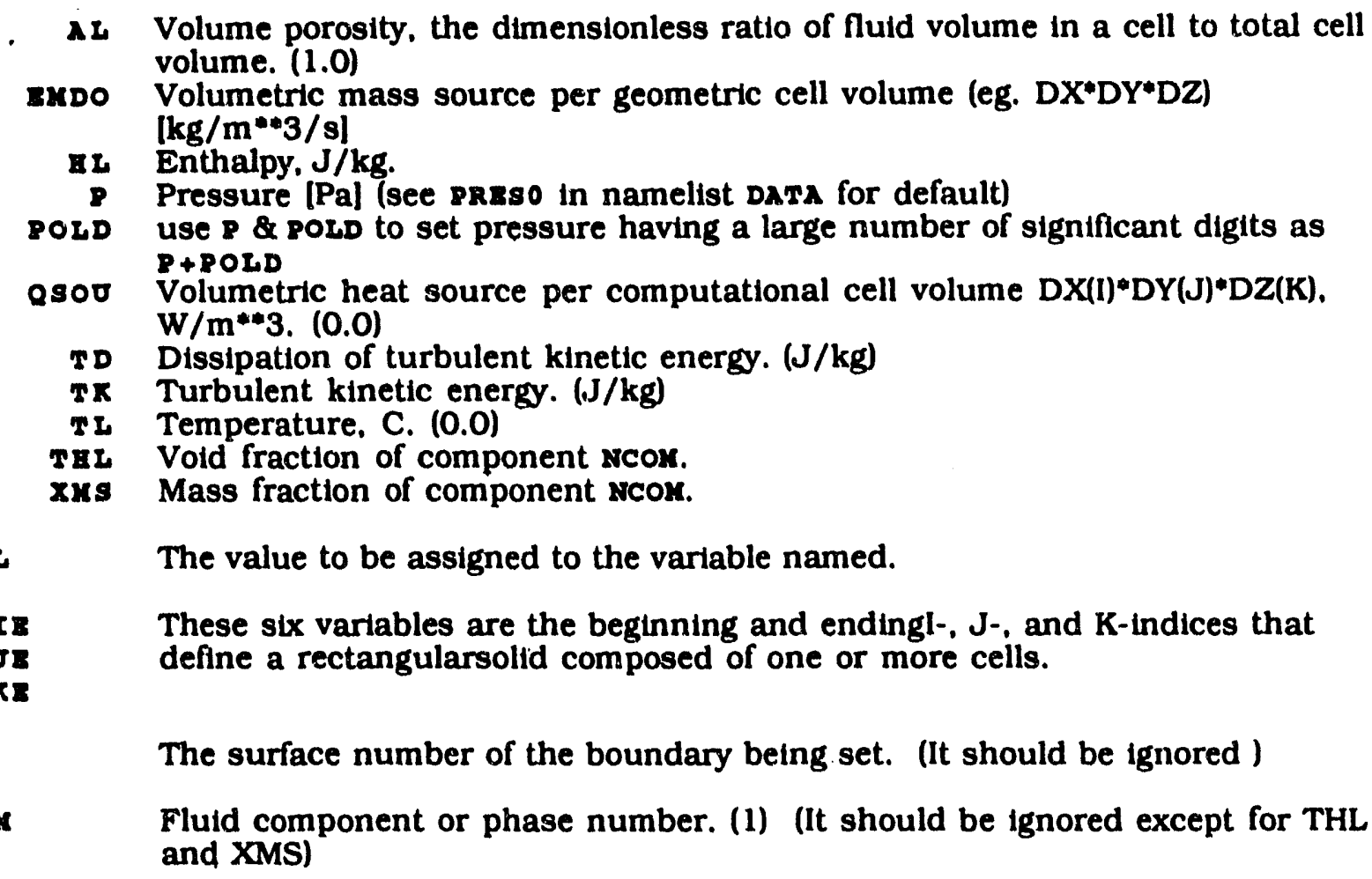

\title{
A.4.9 Coll Surface Initialization Records
}

The purpose of this set of records is to permit initialization of internal cell surface values of any of the arrays listed below. Each card in this section contains the following vartables in the FORMAT $(A 4, F 10.3,914)$.

\section{NAME RVAL IB IE JB JE KB KE}

NAME

ALx Surface permeability, the dimensionless ratio of free flow area to the total surface element area, between cell $(I, J, K)$ and cell $(I+1, J, K)$. (1.0)

ALX Surface permeability, the dimensionless ratio of free flow area to the total surface element area, between cell $(I, J, K)$ and cell $(I, J+1, K)$. (1.0)

$\mathrm{ALz}$ Surface permeability, the dimensionless ratio of free flow area to the total surface element area, between cell $(I, J, K)$ and cell $(I, J, K+1) .(1.0)$

oI U-component of velocity, $\mathrm{m} / \mathrm{s} .(0.0)$

$v i \quad$ V-component of velocity, $\mathrm{m} / \mathrm{s} .(0.0)$

wr W-component of velocity, $\mathrm{m} / \mathrm{s},(0.0)$

RVAr The value to be assigned to the variable named. 
Note. When the location of $\lambda L x(i, j, k), \lambda L Y(i, j, k)$, or $\lambda L z(\lambda, j, k)$ coincides with the location of $\operatorname{Arga}(L)$, then the operative variable is $\operatorname{ARgA}(\boldsymbol{L})$. Similarly, when the location of $\sigma \mathrm{L}(\mathbf{i}, \mathrm{j}, \mathbf{k})$, VL(i,j,k), or wL(1,j,k) coincides with the location of $\operatorname{VBLB}(L)$, then the operative variable is vera(L).

\section{A.4.10 Surface Eloment Initialization Records}

The purpose of this set of records is to permit initialization of surface values of any of the arrays listed below. Velocity boundary condition can be more easily specified using the variable visoc in NAMELIST/DATA/. Each record in this sectiori contains the following variables in the FORMAT (A4,F10.3,914).

NAME RVAL IB IE JB JE KB KE N NCOM

NAME

AREA Boundary surface permeability, the dimensionless ratio of free flow area to the total surface element area.

Fow Mass flow through boundary surface element. [kg/s]

BLB Boundary enthalpy, $[\mathrm{J} / \mathrm{kg}]$.

RLB Boundary density. $[\mathrm{kg} / \mathrm{m} * * 3]$.

TLB Boundary temperature.[C].

TKB Boundary turbulent kinetic energy, $[\mathrm{J} / \mathrm{kg}]$.

TDB Dissipation of boundary turbulent kinetic energy, [W/ $/ \mathrm{kg}]$.

VIL B Magnitude of the velocity normal to the surface in the direction indicated by XNORML (N), YNORML (N), and ZNORKL (N), [m/s].

THLB Boundary vold fraction for component NCOX.

xus B Boundary mass fraction for component wcox.

RVAL The value to be assigned to the variable named.

IB, IE

JB, JE

$\mathbf{K B}, \mathbf{K} \mathbf{z}$

N

ICOK
These six variables are the beginning and ending $\mathrm{I}-, \mathrm{J}-$, and $\mathrm{K}$-indices that define a rectangular solid composed of one or more cells. The rectangular solid that defines or partially defines a surface is the one which is totally interior and adjacent to, or partially interior to and intersecting that surface.

The surface number of the boundary being set.

Fluid component or phase number. (1) (It should be ingorned except for XusB and TEIB)

Note. The scheme to indicate surfaces in the BOUNDARY SURFACE IDENTIFICATION CARDS is the same as that used to indicate surfaces in the BOUNDARY VALUE INITIALIZATION CARDS. This, however, is different from the scheme used to indicate surfaces in the INTERNAL CELL INITIALIZATION CARDS. In the former case. surface elements are indicated by the cell which is adjacent to and on the side pointed to by the surface normal. In the latter case, cell $(\mathrm{I}, \mathrm{J}, \mathrm{K})$ indicates the surface between cell $(I, J, K)$ and either cell $(I+1, J, K)$, cell $(I, J+1, K)$, or cell (I,J,K+1), whichever is appropriate for the variable being initialized. Surfaces lying on boundarles must not be initlalized using the INTERNAL CELL INITIALIZATION CARDS but rather the BOUNDARY VALUE INITIALIZATION CARDS. 


\section{A.4.11 Tube Slde Condenser Model}

This section defines the variables used in the COMMIX condenser calculation when $I T O B E=1$.

\section{A.4.12 NAMELIST/TUBS/}

sroses Total number of the tube channel.

IrXcerL Total number of computational cells in tube side.

DKTB (N) Dlameter of tube in tube channel. Where $\mathrm{N}$ is the tube channel number.

TOBExO (N) Number of tubes in tube channel $\mathrm{N}$.

$>0$ Number of tubes in tube channel $N$ interacting with the associated fluid cell in steam side.

$<0$ The absolute value is the tube number per unit area within the tube channel N.

IXATB Index for computing the fluid properties of the coolant. The value of this index may be any interger from 1 to 22. For ImaxB from 1 to 20, the fluid properties are computed from the fast running simplified straight-line approximation to the state equations. When the value is 21 or 22 , the fluid properties are computed from the rigorous equation-of-state subroutines. It is similar to MATYPI $(X)$ and MATWAL $(X)$ as described in KATERIAL PROPERTIES section. For value of 1 to 20, the following coefficients must be specifled in NAMELIST/DATA/.

COK(IMATB) C1K(IMATB) . C2K(IMATB)

CORO(IMATB) C1RO(IMATB) C2RO(IMATB)

COD(IMATB) CID(IMATB) C2D(IMATB)

COH(IMATB) CIH(IMATB) C2H(IMATB) C3H(IMATB)

COP(IMATB) CIP(IMATB) C2P(IMATB)

COMU(IMATB) CIMU(IMATB) C2MU(IMATB)

\section{A.4.12.1 Iteration Paramotors}

ITERTB Iteration number betweem momentum and energy equation. (1)

EPSTB 6 Convergence criterion. (1.0e-5)

\section{A.4.12.2 Rosistance Coofflcients}

The pressure drop along the tube is expressed in the the following forms:

$D P D X=-F O R C T B * R I * A B S(O L) * O L * F T B / D X T B$

\footnotetext{
where $F T B=A T B L * R B * B T B L+C T B L$

when RE < RIYTRW, and

PTB $=A T B T * R E * * B T B+C T B T$

when $R E>-$ RIYTRS, and

$R I=R I * U_{L} * D X T B / V I S$, and

$R L$ is the local density.
} 
or is local velocity, and

vIs is the local viscosity.

FORCTB Force coefficient.

REYTRA The transition Reynolds number.

ATBI Correlation coefficients when the Reynolds number above.

BTB $\quad R E$, is in the laminar regime,

CTBI 1.e., when RE $<$ REYTRN.

ATBT Correlation coefficients when the Reynolds number above,

BTBT RE, is in the turbulent regime,

CTBT 1.e., when RE >= REYTRN.

\section{A.4.12.3 BoundaryCondition Type}

Inlet and outlet of condenser tubes must have a velocity boundary condition type and a temperature or heat flux boundary condition type.

\$ 1 Inlet surface

2 Outlet surface

RFLOTB (N) Type of velocity boundary condition. (The default for inlet plane is 1 and outlet is -5)

-5 Continuative mass flow outlet.

-2 Continuative velocity outlet.

- 1 Continuative momentum outlet.

1 Constant velocity boundary with velocity set from viLOTB(N).

2 Constant mass flow. FLOW = RLBTB (N) *VELOTB (N) * ARRA

$100+\mathrm{NF}$ Uniform transient velocity boundary with normal velocity set from the product of the NFth transient function and veLOTB(N).

$200+N F$ Transient mass flow boundary with normal velocity set uniformly from VELOTB (N) / (RLBTB (N)*AREA).

KRRITB (N) Type of pressure boundary condition. Pressure boundary conditions are applied to the cells adjacent and interior to the boundary surface specified. (The default for inlet plane is 0 and outlet plane is 1)

$0 \quad$ No pressure boundary condition is applied.

1 Uniform constant pressure boundary with pressure set from PREsrB(N).

$100+\mathrm{NF}$ Uniform transient pressure bnundary with pressure set from the product of the NFth transient function and PRESTB(N).

Ктгхтв (X) Type of temperature or heat flux boundary condition. (The default for inlet is 1 and outlet is 400)

1 Specified constant temperature boundary with temperature set from TEXPTB (N)

$100+N F$ Uniform transient temperature boundary with temperature set from the 400 product of the NFth transient function and $\mathrm{T}$ 


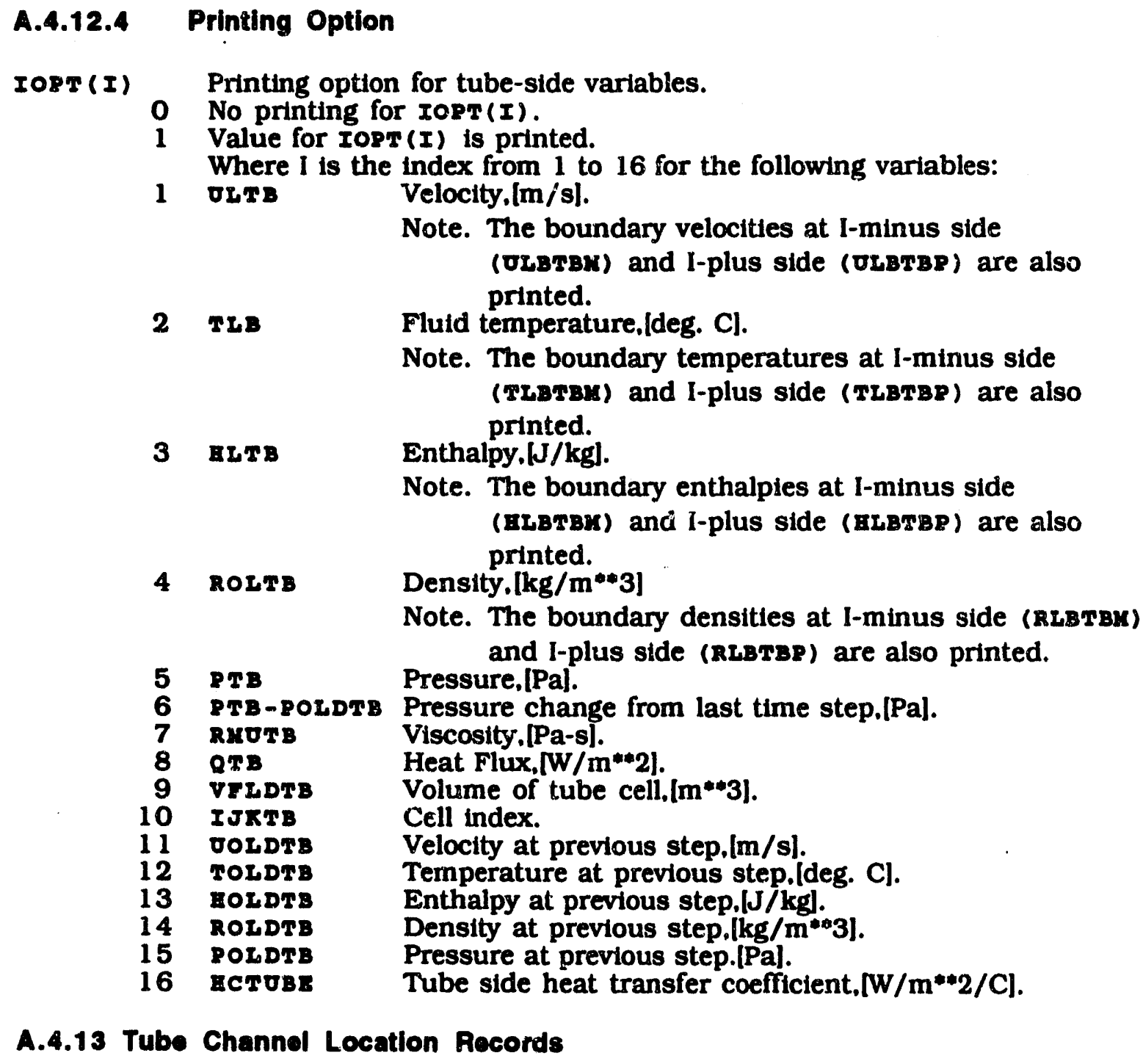

\section{A.4.13 Tube Channel Location Records}

The tube channel location records contain the following variables in FORMAT $(\mathbf{A 4}, \mathbf{8 I 4 )}$

NAM NUM IB IE JB JE KB KE NFD

wax ToB: It indicates the condenser tube channel.

sox Tube channel number.

IB, II These stx variables are the beginning and ending $\mathrm{I}-, \mathrm{J}-$, and $\mathrm{K}$-indices of tube JB, $\mathrm{JI}$ channel wor.

$\mathbf{K B}, \mathbf{K \Sigma}$

NED

The flow direction in the condenser tube.

-1 The flow direction is from $+x$ to $-x$.

1 The low direction is from $-x$ to $+x$.

-2 The rilow direction is from $+y$ to $-y$.

2 The flow direction is from $-y$ to $+y$.

-3 The flow direction is from $+z$ to $-z$.

3 The flow direction is from $-z$ to $+z$. 


\section{A.5 Control Parameters at a Glance}

The table below is included to clarify the role of some of the control parameters. The values indicated, while not guaranteed, are ones that have been found to work in many applications. The user is encouraged to optimize these parameters according to application. A single asterisk indicates that the parameter is used. A double asterisk indicates that the parameter is not used. A value enclosed in brackets 'Il' indicates that the default value is different and that this value must be explicitly specified in the input.

IMPLICIT TIME ADVANCEMENT

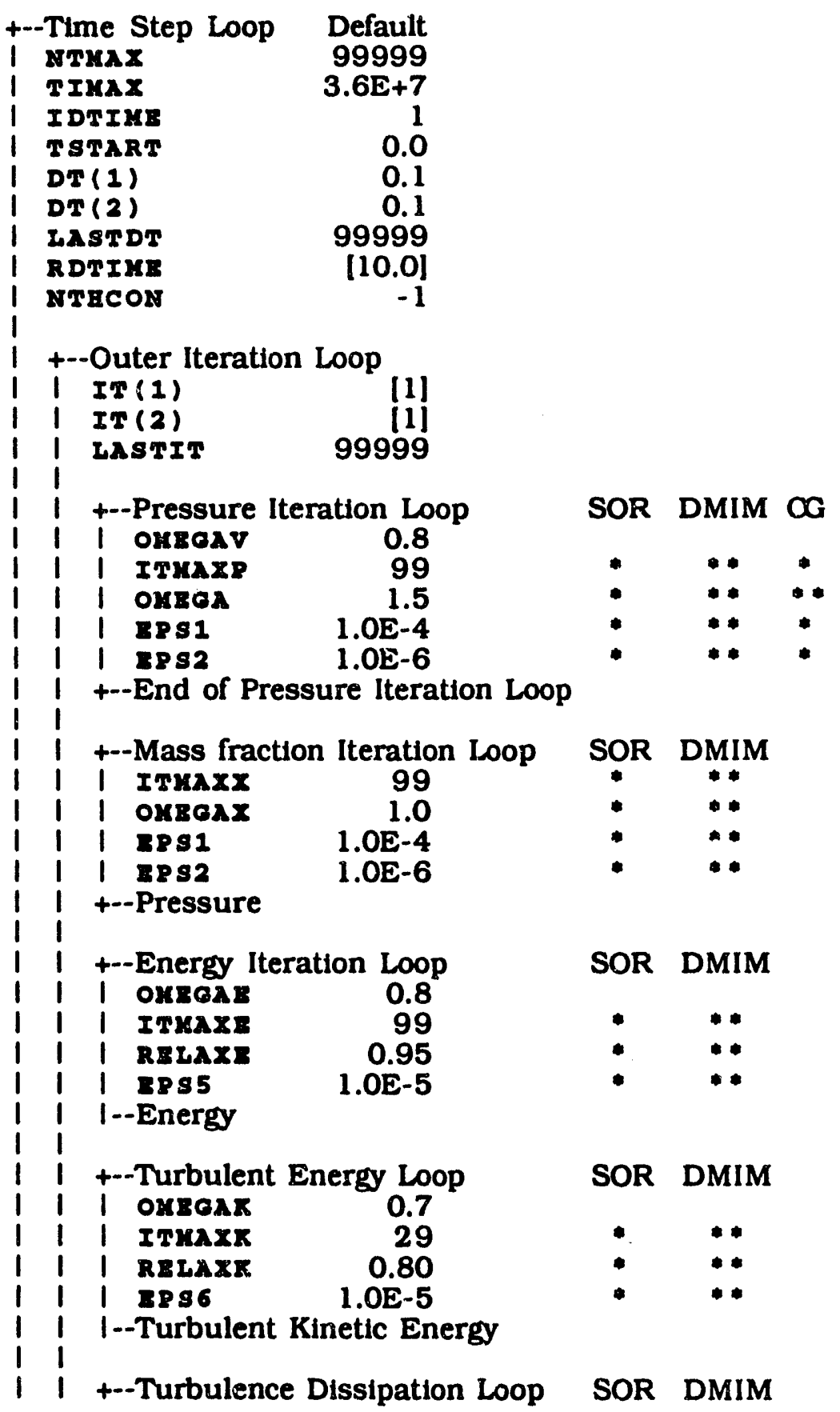

Solver

Default

99999

0.1

9999

0.0

[1]

Loop

99

1.0E-4

re Iteration Loop

mation

SOR DMIM

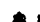




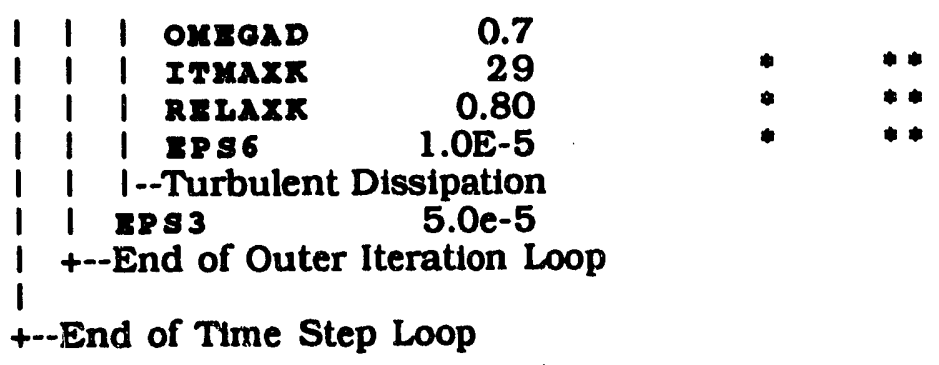

\section{A.6 Steady-State Definition}

Steady-state is reached when the following conditions are met:

1. DL $<1.0$ where

DI=maximum cell residue/DCowv.

Dcosrv =EP\&1*(סvmux $+E P \& 2$ ), and

ovmax is computed in SUBROUTINE GDCONV.

2. ABS (DUMAX/ (VELMAX*ougeAv))<EPs3 where

Dounx is the maximum change of u-velocity component

vewiax is wax(vcowv, maximum u-velocity component).

3. ABS (DVsux/ (VELuAx"OxroAv))<EPS3 where

Dviux is the :.jaximum change of v-velocity component

veriunx is $\operatorname{kax}$ (vcoirv, maximum v-velocity component).

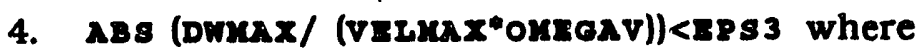

Dincux is the maximum change of $w$-velocity component

veruax is $\operatorname{mx}$ (vcowv,maximum w-velocity component).

5. ABs (DE/ (DELTAE*OKMOAX))<8PS3 where

$\mathrm{Dz}$ is the maximum change in enthalpy over two consecutive steps, and DELTar is $\max$ (DECowr, maximum current enthalpy variation).

\section{A.7 Error Messages}

The following table gives a listing of the error messages processed by SUBROUTINE ERRCHK. It is intended that this section will be ever expanding thus making the running of this code easier. In many cases, relevant information is printed out in the line(s) before the error message block. When appropriate, the error messages below refer to the variables in this information line. Variables are identified by their type ( $R$ for real, I for integer, and $A$ for literal) and their relative position (1 through 12) in the line. For example. A1,R2,13,14,15,16,17,18,19 would be used to refer to varlables printed in the following line:

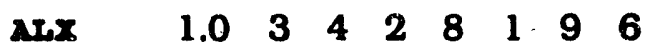


1 RISTAR

2 nтстов

3 घтстов

4 BARIX
In reading the restart file, a block from COMMON /SPACE/ was found to have a length different from that specified on the restart file.

Invalid heat transfer correlation index. Check the index. The correct index should be from 1 to 10 .

Invalid heat transfer correlation number was used. The correct number must be in the range of 71-90.

An invalid input parameter has been encountered while initializing cell or surface element values. There are numerous conditions which may cause such an error. Some of them are listed below. One of the following necessary conditions has not been satisfied:

$$
\begin{aligned}
& 1<=\text { IB }<=\text { IMAX, } \quad 1<=\text { IE }<=\text { IMAX, } \\
& 1<=\mathrm{JB}<=\text { JMAX, } \quad 1<=\mathrm{JE}<=\mathrm{JMAX} \text {, } \\
& 1<\mathrm{KB} \Leftrightarrow \mathrm{KMAX}, \quad 1 \Leftrightarrow \mathrm{KE} \Leftrightarrow \mathrm{KMAX} \text {, } \\
& 1<=\mathrm{N} \Leftrightarrow \text { NSURF, } \\
& 0<=\text { NCOM } \leqslant \text { NCOMP, } \\
& \text { IB }<=\mathrm{IE}, \quad \mathrm{JB}<=\mathrm{JE}, \quad \mathrm{KB}<=\mathrm{KE} \text {, } \\
& 0.0<\text { volume porosity (AL) }<=1.0 \text {, or } \\
& 0.0 \ll \text { surface permeabllity (ALX, ALY, ALZ) }<=1.0 \text {. }
\end{aligned}
$$

The offending line is either printed above the error message box or is indicated with the following string printed at the right of the line: '<--*ERROR**'. In both cases the input line is ignored.

\begin{tabular}{ll}
5 & ETCOFF \\
6 & ETCOEF \\
7 & TBSIDE \\
\hline 8 & TBgIDE \\
& \\
9 & TBSIDE \\
10 & HTCOND \\
11 & BOXIS
\end{tabular}

12 Boxss

Invalid heat transfer correlation index, th, was used. The corret index is from 1 to 10 .

Invalid heat transfer correlation number,ic, was used. The correlation numbers greater than $\mathbf{5 0}$ are not avallable.

The number of cells in tube side has exceeded the value NMCELL as specified in NAMELIST/TUBS/. If the input value is correct check the BOUNDARY SURFACE IDENTIFICATION RECORDS for possible errors.

Errors have been found in the order of the TUBE-CHANNEL LOCATION RECORD. These must be resolved before execution can continue.

An invalid TUBE-CHANNEL LOCATION RECORD has been found. Either an index is out of range, or NFD does not equal to $1,2,3,-1$, -2 , or -3 .

Invalid heat transfer correlation number was used.

One of the indices of the above BOUNDARY SURFACE

SPECIFICATION CARD is outside of one of the following ranges:

$$
\text { I=1,IMAX J=1,JMAX } K=1, \mathrm{KMAX} N=1 \text {, NSURF, }
$$

or one or more of the beginning indices is greater than the corresponding ending index.

$$
\text { l.e., } \mathrm{IB}>\mathrm{IE} \text { or } \mathrm{JB}>\mathrm{JE} \text { or } \mathrm{KB}>\mathrm{KE} \text {. }
$$

While processing the card printed above the error box a surface element was found to be specified as being contained in two 
surfaces. The cell and surface identifiers are $(11,12,13)$ and 14 and I5 on the second line.

13 BOXEs

14

IRRCBK

15

FIIIX

16

GEOX3 D

17

FIIIX

18 FIIIX

19

20 soxss

21 BOx=8

22 FIIIX

23 xIIOC

$24230 x$

25 INITAL

26 INITAx

On BOUNDARY SURFACE IDENTIFICATION CARDS, surfaces must be specified so that surface numbers are in increasing sequential order with all irregular surfaces preceding regular surfaces.

Only fifteen calls to ERRCux are allowed before termination. This number can be increased by changing the value of wcalss in SUBROUTINE ERRCHK.

One of the indices $I, J$, or $K$ is outside of its expected range 1-IMAX, 1-JMAX, or 1-KMAX respectively. This error usually occurs when the BOUNDARY SURFACE IDENTIFICATION CARDS have left a hole in the boundary. Recheck the BOUNDARY SURFACE IDENTIFICATION CARDS for an undefined or incorrectly defined surface and see the appendix section entitled FINDING HOLES IN THE BOUNDARY.

LMPRNT caused termination. Error is caused from error No. 15,17, or 18 in Subroutine rILLX.

The total number of cells counted in Frimk has exceeded the upper bound of IMAX*JMAX*KMAX. Recheck the BOUNDARY SURFACE IDENTIFICATION CARDS.

Excessive wrap around in the THETA direction. Recheck the BOUNDARY SURFACE IDENTIFICATION CARDS. Also assure that all surface normals are pointing into the calculational area.

Time has run out while attempting to number the cells in rILLx. This probably has been caused by an input error in the BOUNDARY SURFACE IDENTIFICATION CARDS.

The number of surface elements has exceeded the value of NLl as specified in NAMELIST/DATA/. If the input value is correct check the BOUNDARY SURFACE IDENTIFICATION RECORDS for possible errors.

The number of cells has exceeded the value of NM1 as specified in NAMELIST/DATA/. If the input value is correct check the BOUNDARY SURFACE IDENTIFICATION RECORDS for possible errors.

The number of cells has exceeded the value of NM1 as specified in NAMELIST/DATA/. If the input value is correct check the BOUNDARY SURFACE IDENTIFICATION RECORDS for possible errors.

Changes in NM1, NL1, IMAX, JMAX, and KMAX are not allowed when restarting (IFRzS $=2$ or IFRES $=3$ ).

When using the Simplified Properties Option you must input nonzero values for $\mathrm{FOH}, \mathrm{ClH}, \mathrm{CORO}$, and $\mathrm{COK}$. Be aware that the Simplified Properties Option computes properties as a linear function of temperature only and as such must be used with extreme caution!

The duct wall model is not available in this version. Use $\operatorname{Krmax}(N)=400$ and Thermal Structure to model the equivalent thermal effects.

A nonpositive value of TExPo has been found in INITAL. Set TruPo to some positive value in NAMELIST/DATA/. 
27 INYORC

28 ENLOOP

29 FORCIS

30

Forcss

31 FORCES

32 INTTAL

TSAREA

34 INITAI

35

OOTPT

36

TSAREX

37

TSAREA

38

MXI

39

พละ

43

TSCAN

44 INP STR
An invalid input card has been encountered in SUBROUTINE INFORC while reading the FORCE STRUCTURE SPECIFICATION RECORDS. The first field must contain either 'XFOR', 'YFOR', or 'ZFOR'. The indices IB and IE, JB and JE, and $K B$ and $K E$ must be in the ranges 1 through IMAX, 1 through JMAX, and 1 through KMAX respectively. The invalid card printed above the message is ignored and execution continues.

Set ISKEW $=0$ or 2.

An invalid correlation was specified for a force structure. Execution continues after setting zroRCE $=-1.0 e+20$.

A Negative velocity was returned from the subroutine to to determine the velocity through a valve. Execution terminates.

An invalid valve position was specified in the variable IVALVB. Execution continued after setting $I V A L V B=0$ and FORCs $=-1.0 E+20$.

Ismatr has been found to be 0 while attempting to restart from a previous run. It has been reset by the code to 1 . Verify that this is an acceptable fix. Execution continues.

RODF has a value of zero in the thermal structure prototype input.

NL1 and wM1 must not be specified in NAMELIST /GEOM/ when restarting from a previous run with ISTATs $>0$. Remove NL1 and mI from NAMELIST/GEOM/ and rerun.

The value $I 1$ is an invalid value of ISTPR or NYHPR. specifically, the vv field is not defined. The value is ignored and processing continues.

The value of Iosox is invalid. Set Iosox to 0 or -1 and rerun.

A negative material value has been found in the thermal structure input.

The liquid water property function waLTsג has been encountered a negative pressure when attempting to compute the saturation temperature.

The liquid water property function warssa has been computed a negative saturation temperature.

On of the following input rules for thermal structures has been violated: TYPI namelists can only appear first, after FLOID namelist and after MATrRIAL namelists. The geometrical characteristics, IXYz, must be one of the following values: $1,2,3,11,12,13,101$. 102. 103. FUOID namelists can only appear after TYRI and wringal namelists. Each thermal structure must have at least one matertal region.

When computing areas and volumes of the partitions of the thermal structure material regions an inner radius was found to be less than $-1.0 E+4^{*}$ OUTR, where OOTR was te outer radius as specified on the TYPI card. Check the thermal structure input for OUTR, DRPAR, and MUPAR. If no errors are found here check the entire thermal structure prototype input. The negative radius is reset to zero and execution continues. See error number 45 for a list of the variables printed above the error block.

When computing areas and volumes of the partitions of the thermal structure materlal regions an inner radius was found to be larger than the outer radius. Check the thermal structure input. The inner radius is reset to the outer radius end execution continues. 
The parameters printed above the error block are: $\mathrm{N}$ - structure number, IREO - region number, IPAR - partition number, OUTR (N) - outer radius, DR - region size, ROOT - outside radius, RIN - Inner radius.

An invalid THERMAL STRUCTURE LOCATION card has been found. Either an index is out of range, or the Loc value is invalid (must be either 'OOT' or 'IN'), or the sor value does not match the number of any THERMAL STRUCTURE PROTOTYPE.

A THERMAL STRUCTURE PROTOTYPE has been encountered which has fluid cells interacting at both outside and and inside surfaces, however, the number of cells interacting with the outside surface does not equal the number of cells interacting with the inside surface. 11, 12, 13, and 14 are the structure number, surface interaction code, number of cells interacting with surface 1 , and number of cells interacting with surface 2 .

THERMAL STRUCTURE PROTOTYPE RECORDS are inconsistent with the THERMAL STRUCTURE LOCATION RECORDS. Either the TSP records specify only cells interacting with the outside surface and the TSL records specify some cells interacting with the inside surface, or the TSP records specify only cells interacting with the inside surface and the TSL records specify some cells interacting with the outside surface. The values printed above the error message are the same as those identified in error 47.

The THERMAL STRUCTURE LOCATION RECORD printed above the error message box indicates a cell within the ranges of the indices which is not a valid calculational cell. The specific $I, J$, and $K$ indices are printed out as 19, 110, and 111 . This cell is ignored and execution continues. The results which follow are likely incorrect.

An invalid value of scoror has been encountered. scorox must have a value of $8,9,10$, or 11 . Execution continues without printing this table.

The BOUNDARY SURFACE IDENTIFICATION CARDS have defined a single sided interior boundary surface between cells II and 12 . Check to see that all surfaces you have defined bound calculational cells. Also be sure that any interior surface has calculational cells on both sides of it. Reread the BOUNDARY SURFACE IDENTIFICATION CARD input section and check your input. Execution continues however subsequent results are questionable.

An invalid value of IXYZ has been found in the thermal structure prototype input.

Currently only $1 \mathrm{CO}$ thermal structure prototypes are allowed. If more is needed, 'hanges must be made in COMMON /REBALS/ in subroutine ALLCIC and INPSTR. Execution terminates.

Errors have been found in the order of the thermal structure prototype input. These must be resolved before execution can continue.

Invalid material number is specified in marras.

The temperature range for indicated material number has been set to $50.0-90.0$ degrees. This can be specified by the user in variables TABLOT and TABEIT.

ZPROPs Invalid materlal number is used. 
Irregular surfaces must have no flow or pressure boundary condition applied across them. That is, $\operatorname{xFLOW}(x)=1$, VELOC $(N)=0.0$. AND KPRES(N) $=0$ for all irregular surfaces $N$. When specifying transient boundary conditions, the transient function number, NF, must be nonzero. That is, KF LOW $(\mathrm{N})=100, \operatorname{xTExP}(\mathrm{N})=100$, $\operatorname{KTEUP}(\mathrm{N})=300$, and KPRES $(\mathrm{N})=100$ are all invalid boundary condition types. Valid types, assuming properly defined transient functions, would be, for example, xrLOW $(*)=101$ and $\operatorname{KTIMP}(\mathrm{N})=102$.

59 oxox3 $D \quad D X(I)$, DY $(J)$, and $D z(x)$ must be nonzero for $I=1$ through Ixax, $\mathrm{J}=1$ through oux, and $\mathrm{K}=1$ through $\mathrm{kmax}$.

60 Grox3 D

The length of the normal vectors defined by xororur (N), YNORML (N), and zNORML (N) must be within one percent of 1.0.

61 INTORC

A previously defined force structure location has been overwritten. In the preceding lines, Al shows the cell face on which the force structure was being defined, 12 is the force structure number, and 13. 14, and I5 give the cell indices. The last force structure defined at a cell face will be the one which is used.

62 INITAL Incorrect values have been specified for ISETEN and/or ISETYo. See NAMELIST/DATA/ for a description.

63 IXITAL New thermal structure input will be read. All previous thermal structure information from previous runs will be ignored.

64 INITAL

New force structure information will be read. All previous force structure information from previous runs will be ignored.

\section{A.8 Storage Allocation}

In order to ease the task of creating load modules (binary files) to fit the size of the problem being considered, a quasi-dynamic storage allocation scheme has been implemented. Space for most of the geometry dependent variables is allocated in the variable S of COMMON /SPACE/. The address of each variable is computed at the beginning of each run. These addresses are then passed into called subroutines where the variables are named and variably dimensioned.

The following table shows a list of the variables with space allocated in variable $\mathbf{S}$ of COMMON /SPACE/. The dimension of each variable is indicated as is the index of variable IS which contains the $S$ offset address of the varlables. If any changes are made in this area, one must assure that consistency is maintained.

\begin{tabular}{|c|c|c|c|c|c|c|}
\hline $\begin{array}{l}\text { VARIABLE } \\
\text { DIMENSION }\end{array}$ & $\begin{array}{l}\text { VARIABLE } \\
\text { NAME }\end{array}$ & INDEX & $\begin{array}{l}\text { VARIABLE } \\
\text { NAME }\end{array}$ & INDEX & $\begin{array}{l}\text { VARIABLE } \\
\text { NAME }\end{array}$ & INDEX \\
\hline PRIMARY & XIP & 1 & KIX & 2 & KJP & 3 \\
\hline GEOMTTRY & KJ & 4 & XXP & 5 & $\mathbf{x x} \mathbf{x}$ & 6 \\
\hline \multirow[t]{5}{*}{ SM 1} & IJX & 7 & & & & \\
\hline & ALX & 13 & $\operatorname{ALY}$ & 14 & ALz & 15 \\
\hline & $\mathbf{A L}$ & 16 & & & & \\
\hline & VILOID & 111 & ATLOX & 112 & AFLOY & 113 \\
\hline & AILOZ & 114 & & & & \\
\hline SII 1 & $\mathbf{M B}$ & 42 & ISORE & 43 & ARSA & 44 \\
\hline
\end{tabular}




\begin{tabular}{|c|c|c|c|c|c|c|}
\hline PRIXARY & EOID & 10 & RL & 17 & OI & 18 \\
\hline FLOID & VI & 19 & MI & 20 & EXDOT & 115 \\
\hline \multirow[t]{6}{*}{ MMTT } & FLowx & 171 & FIOMY & 172 & FLOWz & 173 \\
\hline & EL & 22 & Tr & 23 & RLOLD & 24 \\
\hline & OLOLD & 28 & QSOOR & 29 & VIOLD & 30 \\
\hline & WLOLD & 31 & POID & 35 & $\mathbf{P}$ & 41 \\
\hline & $\mathbf{x x}$ & 177 & ExIOLD & 178 & SOLD (BLT, & 108 \\
\hline & DIFT, DIFEA & 116 & & & TKT, TDT) & \\
\hline \multirow[t]{3}{*}{ XLFT } & VELBN & 46 & $\operatorname{ALB}$ & 48 & RLB & 45 \\
\hline & QBN & 47 & TIB & 49 & & \\
\hline & ILOWB & 176 & $\mathbf{E K R B}$ & 179 & & \\
\hline SECONDARY & OEATI & 25 & VEATL & 26 & WEATL & 27 \\
\hline FIOID & DOOI & 32 & DVOL & 33 & DWOL & 34 \\
\hline \multirow[t]{4}{*}{ NMFT } & $\triangle 00 \mathrm{~L}$ & 38 & AVOL & 39 & AพOL & 40 \\
\hline & $\operatorname{AC1}$ & 67 & $\operatorname{Ac2}$ & 68 & Ac 3 & 69 \\
\hline & Acs & 70 & $\operatorname{Ac} 5$ & 71 & AC 6 & 72 \\
\hline & Aco & 73 & BCO & 74 & SPERAT & 120 \\
\hline TERTIARY & RXO & 8 & DL & 21 & BEOLD & 98 \\
\hline FLOID & BOOID & 99 & BVOLD & 100 & BWOLD & 101 \\
\hline \multirow[t]{2}{*}{ NuFT } & DRDP & 102 & DRDE & 103 & DEDP & 164 \\
\hline & scal & 104 & SPEL & 105 & $c x$ & 50 \\
\hline \multirow[t]{3}{*}{ мист } & XXs & 109 & XYSOLD & 110 & & \\
\hline & TEL & 78 & TELOLD & 79 & & \\
\hline & RTE & 152 & RTEOLD & 153 & & \\
\hline ALCT & rusB & 81 & TELB & 150 & RTEB & 151 \\
\hline \multicolumn{7}{|c|}{ TURBULENCE MODEL } \\
\hline \multirow[t]{4}{*}{$\operatorname{MK1}$} & TROID & 12 & $\mathbf{T} \mathbf{x}$ & 36 & TORVIS & 37 \\
\hline & TORCON & 76 & TD & 80 & & \\
\hline & TDOLD & 137 & BROLD & 138 & BDOID & 139 \\
\hline & sCRI & 106 & SPKL & 107 & & \\
\hline NL1 1 & TXB & 51 & TDB & 136 & & \\
\hline
\end{tabular}

SKEW UPWIND MODEL

\begin{tabular}{|c|c|c|c|c|c|c|}
\hline \multirow[t]{2}{*}{ NFORCE } & ICORR & 60 & CLENTE & 61 & $R E Y L E N$ & 62 \\
\hline & IroRCE & 9 & PORCE & 63 & & \\
\hline \multirow[t]{2}{*}{ MTSEP } & ITsCB & 52 & EYDRA 1 & 53 & EYDRA2 & 54 \\
\hline & RODFR & 55 & YRON & 117 & RODDK & 118 \\
\hline NPAR & STAREA & 56 & STVOL & 7 & & \\
\hline VREO & MATERL & 58 & $\mathbf{E} \boldsymbol{X x}$ & 59 & $\operatorname{sIzZ}$ & 64 \\
\hline NSOR & ICEI & 65 & BSTREL & 66 & ICTOBE & 119 \\
\hline \multirow[t]{2}{*}{ NTts } & TTS & 77 & & & & \\
\hline & T\$T & 83 & TCs & 84 & QFX & 85 \\
\hline
\end{tabular}




\begin{tabular}{|c|c|c|c|c|c|c|}
\hline & gros & 86 & ETs & 87 & NROW & 117 \\
\hline & RODDX & 118 & DIFF & 122 & PSAT & 123 \\
\hline & BF012 & 124 & EXDOT & 79 & & \\
\hline SOR & MREB & 11 & MSKEEP & 82 & & \\
\hline \multirow[t]{3}{*}{ Y SXP } & I $\mathbf{X}$ & 127 & MPERX & 128 & MPINV & 129 \\
\hline & JA & 130 & MWEAT & 131 & $\mathbf{\lambda}$ & 132 \\
\hline & RSP & 133 & IIAF & 134 & IJAF & 135 \\
\hline \multirow[t]{4}{*}{ PCe } & $\mathbf{x} x$ & 88 & KD & 89 & sג & 90 \\
\hline & MWEגT & 91 & $\mathbf{R}$ & 92 & $\boldsymbol{J A}$ & 93 \\
\hline & $\mathbf{A P}$ & 94 & $\mathbf{P}$ & 95 & $Q R$ & 96 \\
\hline & AQR & 97 & A. & 125 & L. & 126 \\
\hline MIJX & DOXXY 1 & 75 & & & & \\
\hline MILIJK & IDOKI & 140 & & & & \\
\hline $\operatorname{ImxAx}$ & IDOK2 & 141 & & & & \\
\hline MIJK & IDOX 3 & 142 & & & & \\
\hline NLI 1 & IDOX4 & 143 & & & & \\
\hline $\operatorname{MAx} 1$ & RDOK1 & 144 & & & & \\
\hline $\operatorname{xax2}$ & RDOK2 & 145 & & & & \\
\hline \multirow[t]{5}{*}{ NMCELL } & OLTB & 180 & PTB & 181 & TLTB & 182 \\
\hline & ALTB & .183 & ROLTB & 184 & RMOTB & 185 \\
\hline & QTB & 186 & VรLDTB & 187 & ROLDTB & 188 \\
\hline & OOLDTB & 189 & POLDTB & 190 & EOIDTB & 191 \\
\hline & DPTB & 192 & IJXTB & 193 & TOLDTB & 194 \\
\hline XTOBES & LFDTB & 195 & & & & \\
\hline
\end{tabular}

\section{A.9 Calling Sequence}

The following table indicates the code structure by showing the calling sequence of the subroutines. Calls to the properties routines and function are not indicated. Also multiple calls may not be indicated.

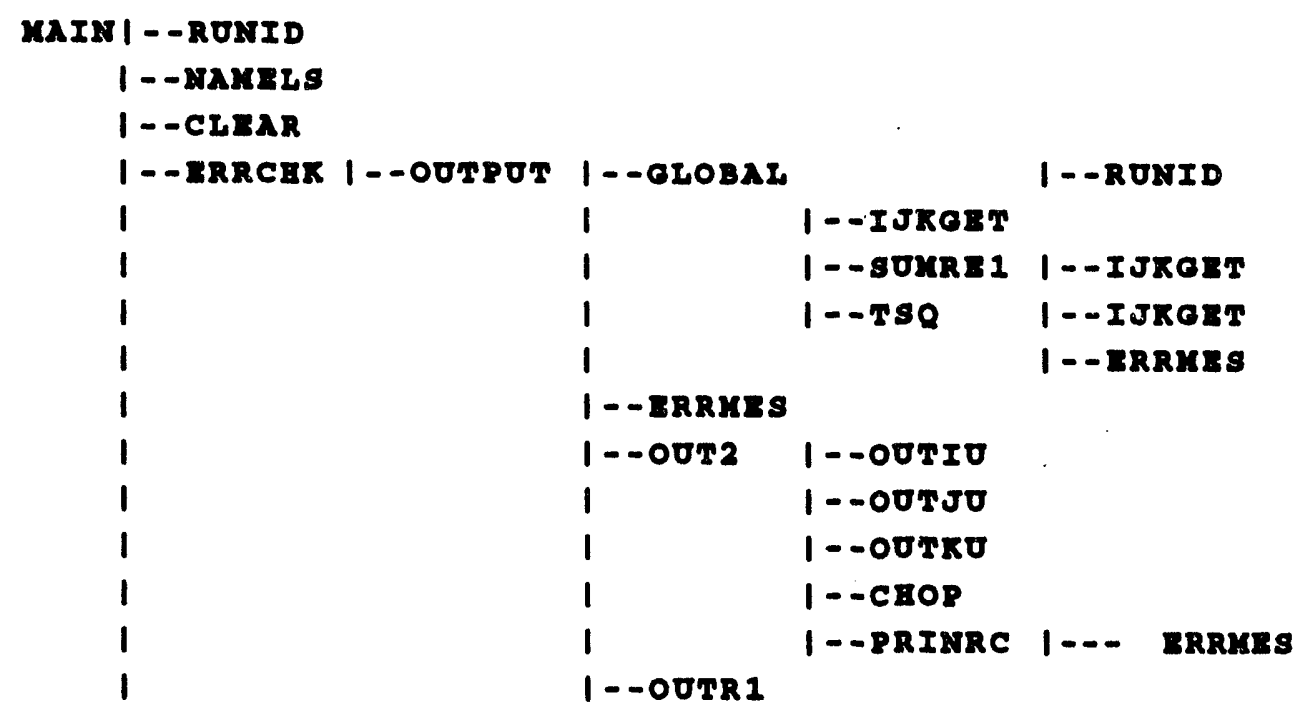




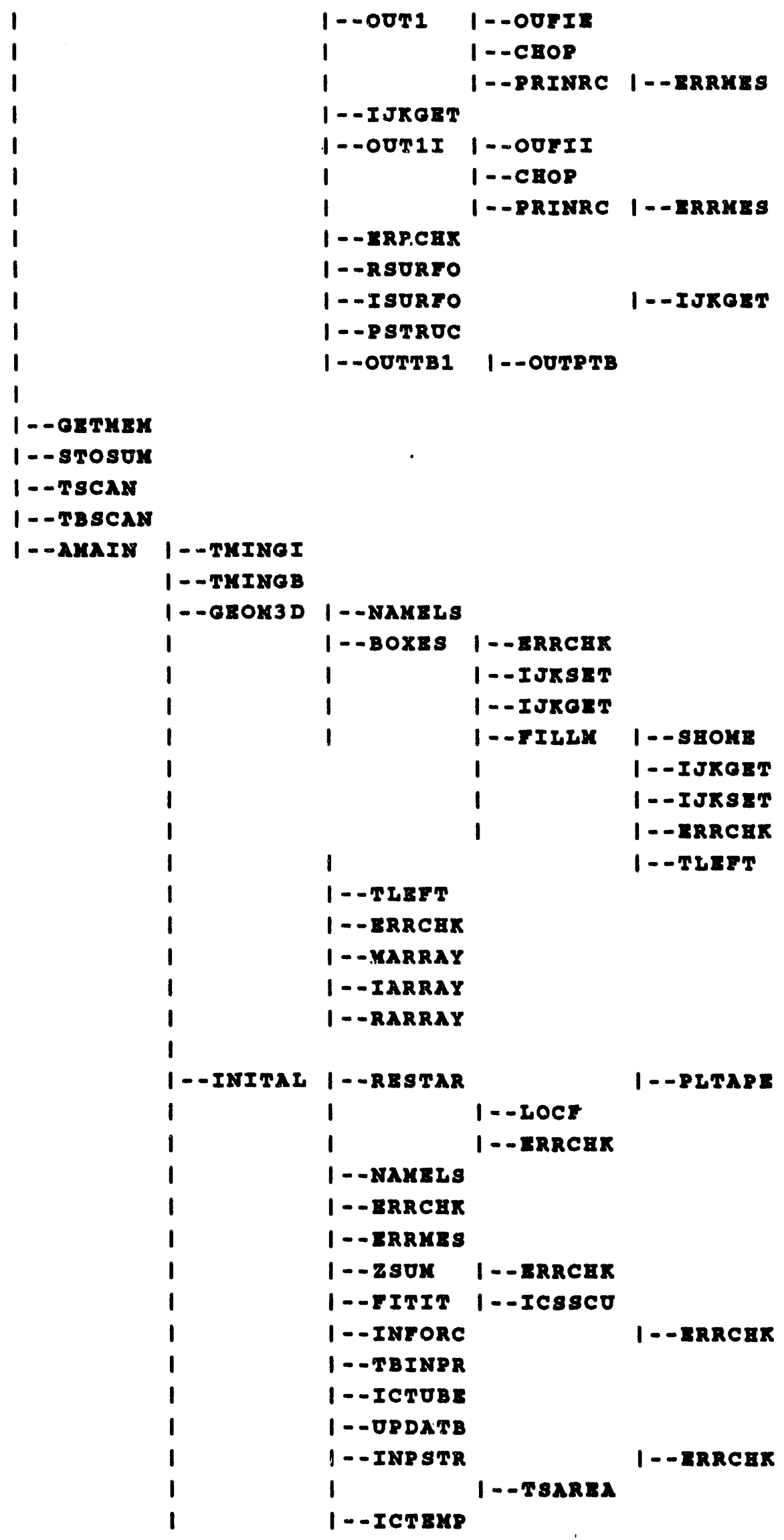




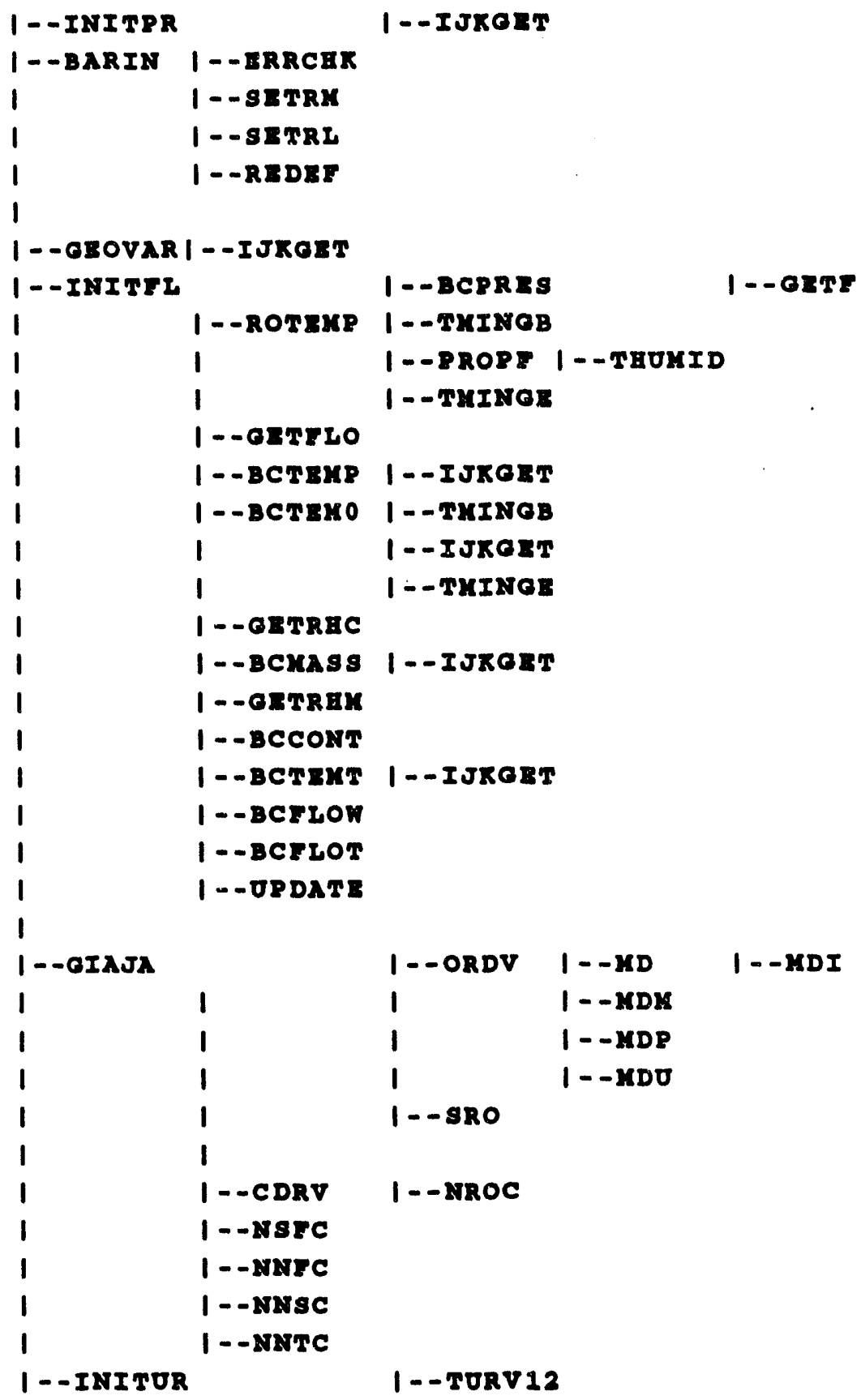

1--RSTROC 1 --TXINGB

| - IJKGET|

1--ATCOEF 1--TMINOB

1 1--ERRKIS

I 1 --RTCOND 1--TMINOB

I 1 1--IRRMIS

1 I 1--TKI*GE

I 1--TKINOE

1

| - - втстбв| - -титмов

1 |--IRRMES 


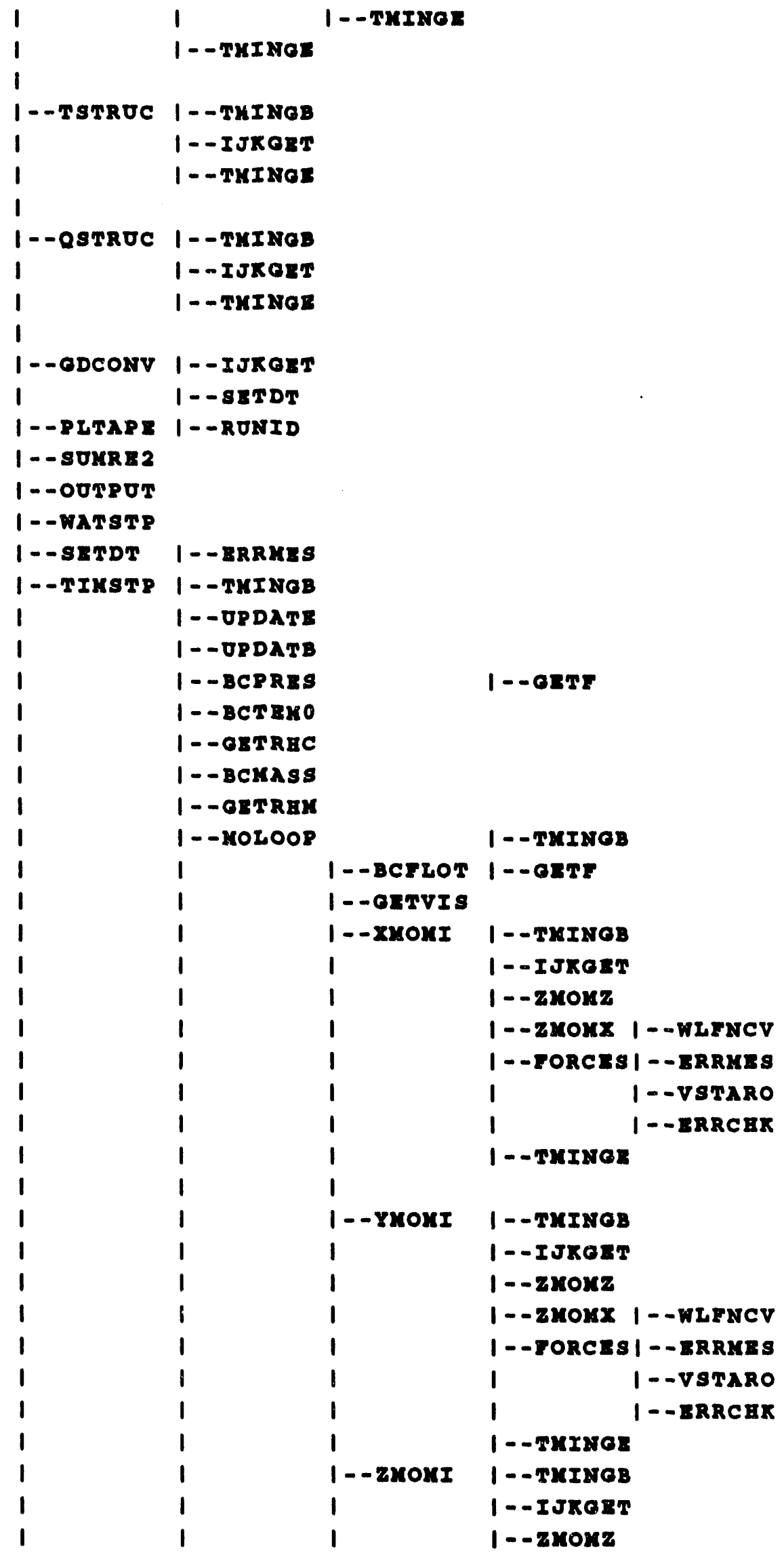




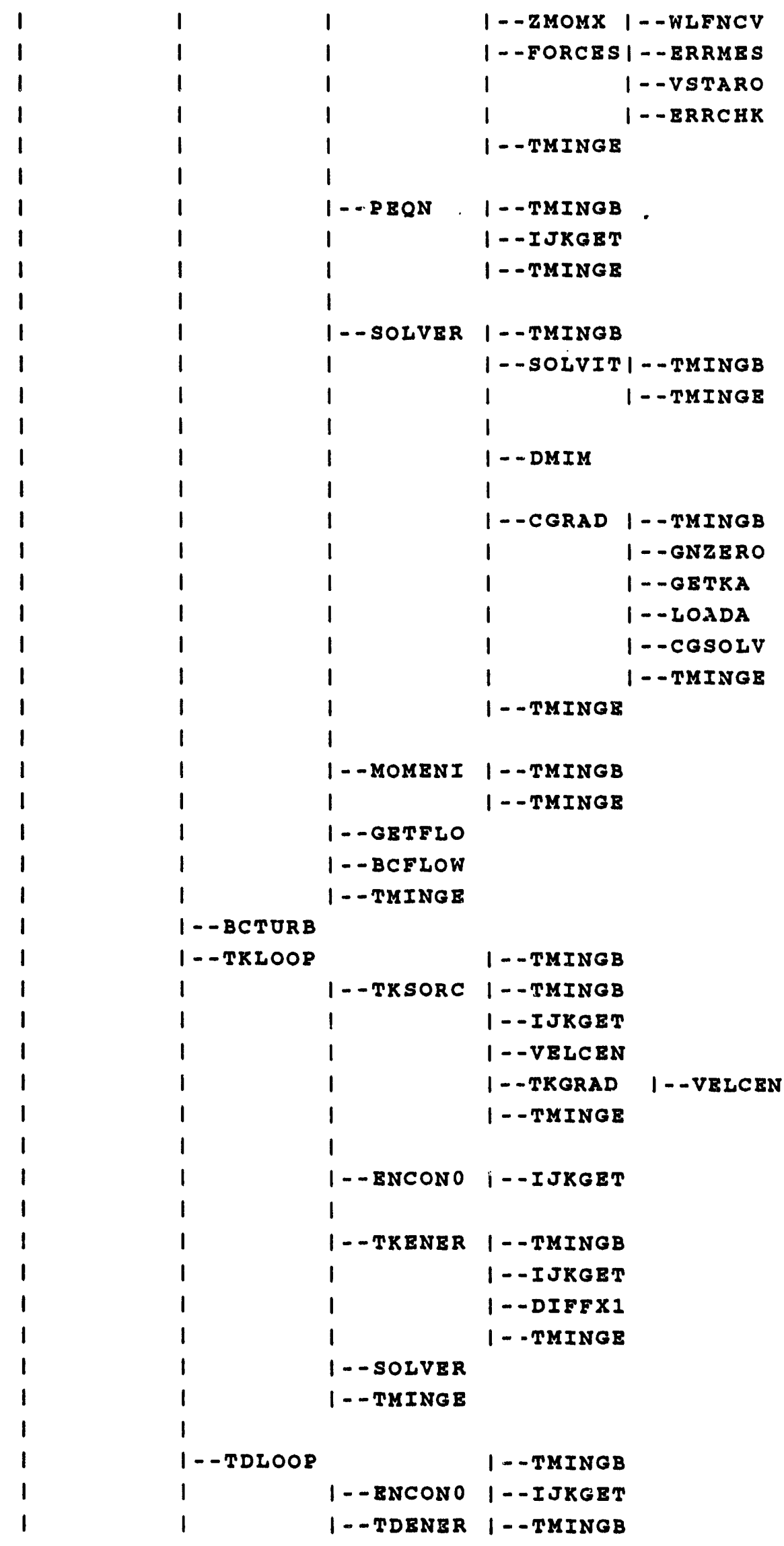




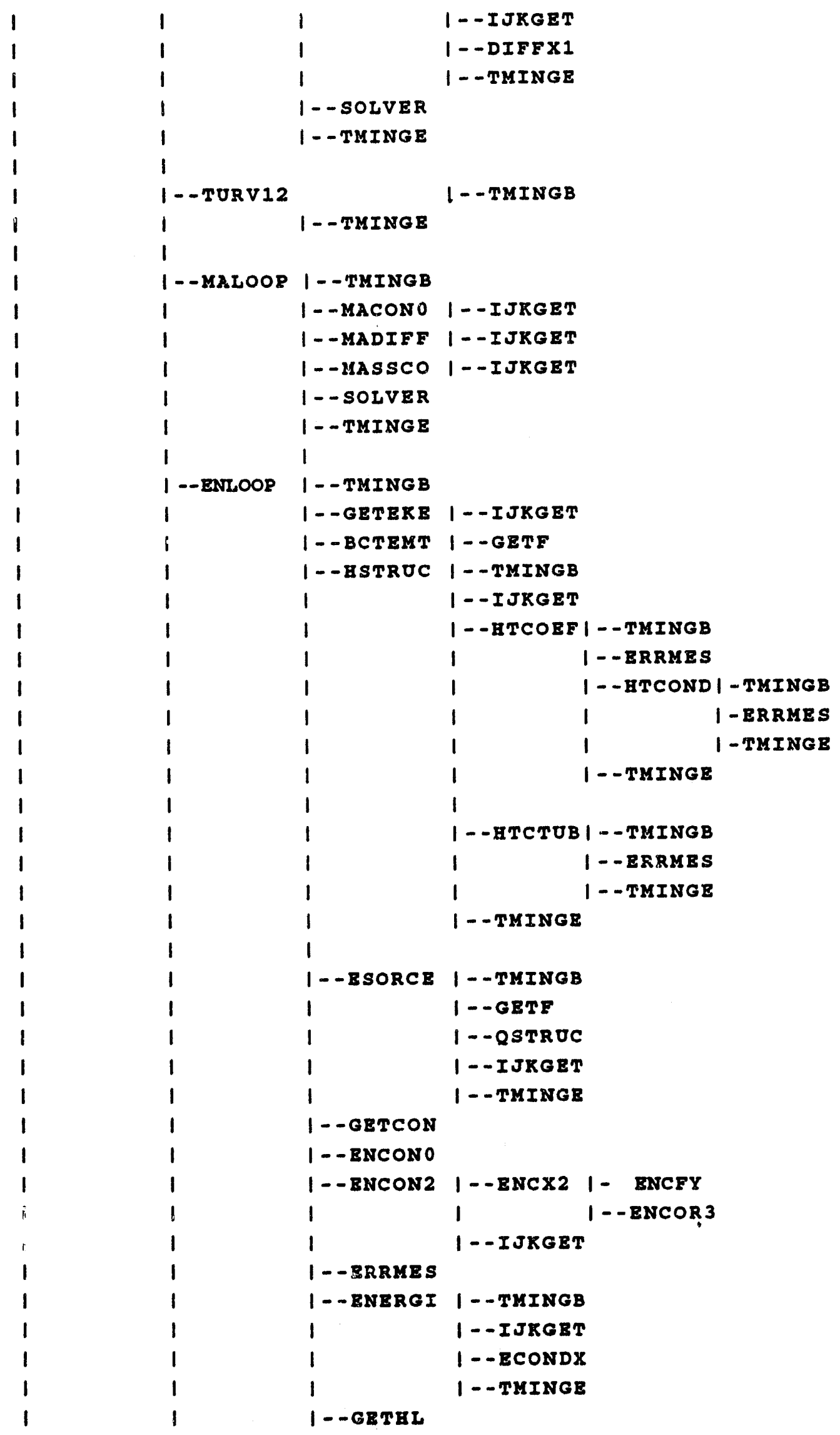




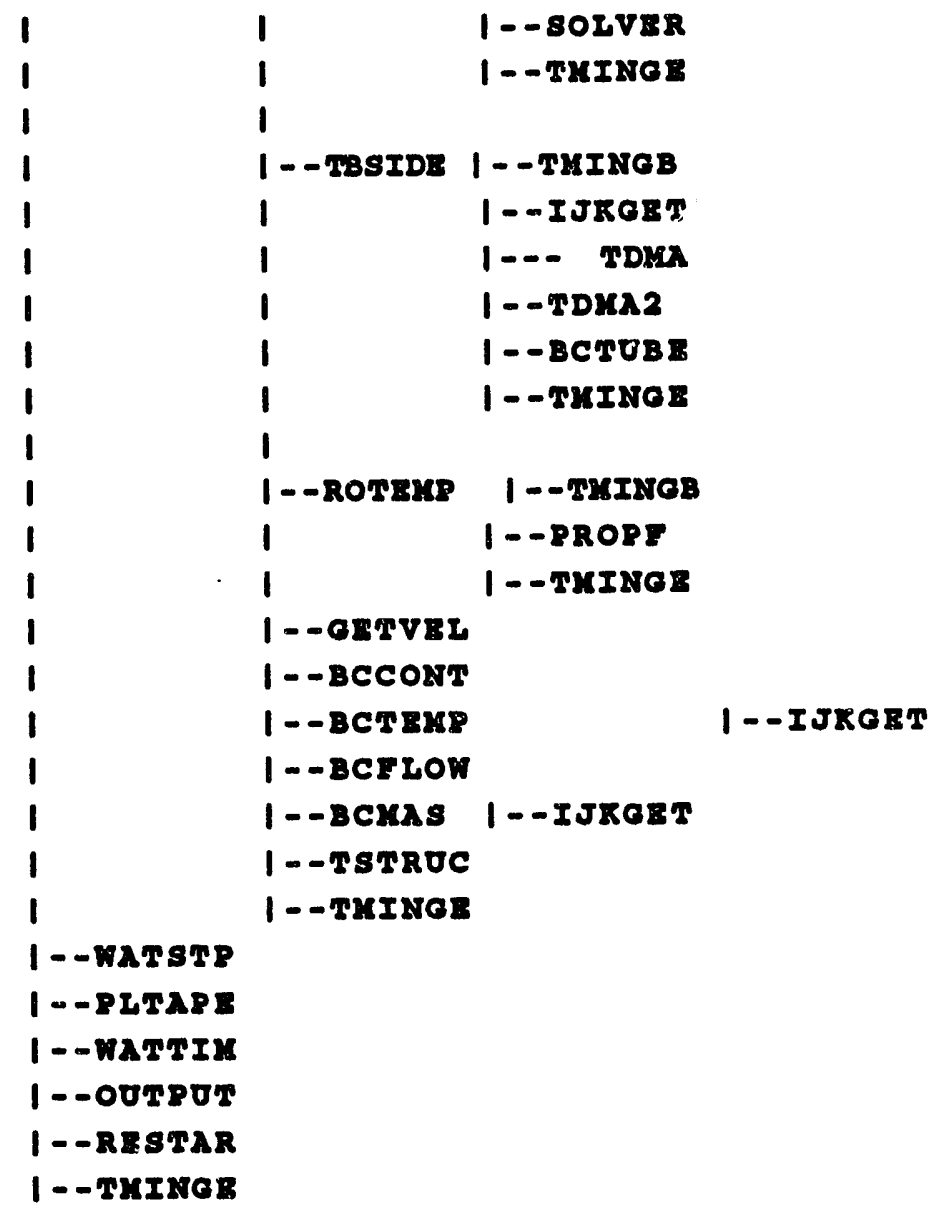

\section{A.10 Overlay Structure}

This section gives one possible overlay structure. If this is found to be insufficient one can use the preceding section to help redesign an alternative scheme. When using a virtual memory operating system, user defined overlays are probably undesirable.

INSERT MAIN,ERRCHK,LOCF

INSERT I4VAR,R4VAR,I4ARY,R4ARY,SPACE

INSERT MACHIN,REBALS,CCORR,CHEATC,ADDCON

OVERLAY ONE

INSERT CLEAR,TSCAN,TBSCAN

OVERLAY ONE

INSERT AMAIN,TLEFT,RARRAY,RESTAR,PLTAPE

INSERT BCFLOT,BCFLOW,BCPRES,BCTEMP,BCTEMT,BCTEMO,BCTUBE

INSERT GETF,GDCONV,WATTIM,WATSTP 
INSERT HSTRUC,QSTRUC,TSTRUC,HTCOND,HTCTUB

OVERLAY TWO

INSERT GEOM3D,FILLM,MARRAY,LARRAY,SHOME

OVERLAY THREE

INSERT BOXES

OVERLAY TWO

INSERT INITAL,FITIT,ICSSCU

INSERT ICTEMP,BARIN,RSET3,RSET2,REDEF,ICTUBE

OVERLAY THREE

INSERT INFORC

OVERLAY THREE

INSERT INPSTR,TBINPR

OVERLAY TWO

INSERT INITZ1

OVERLAY TWO

INSERT TIMSTP,FORCES

INSERT TKLOOP,TSHEAR,TKSORC.TKENER,SOLVEN,BCTURB

OVERLAY THREE

INSERT MOLOOP,XMOMI,YMOMI,ZMOMI,PEQN,GETDL,TDMA,WLFNCV

INSERT SOLVIT,MOMENI,ENLOOP.ESORCE,ENER.AII

OVERLAY THREE

INSERT MALOOP,TBSIDE

OVERLAY THREE

INSERT OUTPUT,RSURFO,ISURFO,PSTRUC

OVERLAY THREE

INSERT INTURB

ENTRY MAIN

NAME G 


\section{A.11 Machine Dependent Routines}

Two machine dependent functions are used in this package.

\section{LOCF}

This function returns the absolute address of the variable which is passed as the argument. It is used both in determining the length of blocks to be written to the restart file and in performing initialization. This function is used extensively in the code and thus its functional equivalent must be supplied when implemented on other systems. An assembly language listing of LOCF for the IBM maching is given below.

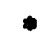

$\bullet$

$\bullet$

LOCF CSECT

SAVE $\quad(14,12), . L O C F$

L

SLL

SRL

MVI

SR

BR

END

$I=\operatorname{LOCF}(X)$

$0,0(1)$

0,1

0.1

12(13), $X^{\prime} F F^{\prime}$

15,15

14
Return location of a variable as 32 big integer.

Load the address.
Remove the sign bit.

Signal return.

Return code.

Return.

\section{TLEFT}

This function returns the CPU time left in the current run in units of 0.01 seconds. This time starts at the time specified on the JOB card and ends at zero when the job is terminated by the system. It is used for timing and to determine when to terminate and write a restart file. For interactive systems the following function may be substituted. This will eliminate meaningful timing measures and the MAXTIME restart capabilities; however it will not effect the codes results.

FUNCTION TLEFT (TIME)

DATA T / $100000.0 /$

$T=T-2.0$

TLEFT $=T$

RETURN

END

\section{A.11. Finding Holes in the Boundary}

The BOUNDARY SURFACE SUMMARY is intended to ald the user in finding holes in the boundary surfaces. It is obtained by setting IBSBDO in NAMELIST/GEOM/. The boundary surface summary consists of two parts. First is a table of binary strings and their 
corresponding printed character. Following this table are skax planes with each calculational cell being represested by one of the characters from the first table. The binary string associated with each character indicates the location of the surface elements in the following way. Each bit in the binary string corresponds to a face of the calculational cell. The first bit corresponds to the face in the I minus (I-) direction. This is the surface between cell $(I, J, K)$ and cell $(I-1, J, K)$. The second bit corresponds to the face in the It direction, the third in the $\mathrm{J}_{-}$, the fourth in the $\mathrm{J}_{+}$, the fifth in the $\mathrm{K}-$, and the sixth in the $\mathrm{K}+$ direction. A surface element is defined at a cell face if the bit corresponding to that face has a value of 1 .

For example, suppose " $\mathrm{F}$ " is printed at the location for cell $(1, \mathrm{~J}, \mathrm{~K})$. "F" corresponds to the binary string "011000". This indicates that a surface element has been defined in the $I+$ and $J$-directions, that is, between cells $(I, J, K)$ and $(I+I, J, K)$ and between cells $(I, J, K)$ and $(\mathrm{I}, \mathrm{J}-\mathrm{I}, \mathrm{K})$.

In order for this scheme to be effective sic table should contain 62 different printable characters. A blank corresponds to string "000000" and string "111111" should never occur. While we have a laser printer with both upper and lower case at ANL, the printers usually used are impact printers with only about 58 different characters. Therefore, the current implementation uses the character "?" to correspond to all of the following binary strings: "111110", "111101", "111011", "110111", "101111", "011111", and "111111". This does introduce some ambiguity however the impact is probably not serious. For those users who wish to eliminate these duplications, changes must be made in SUBROUTINE SHOME. 
Appendix B: List of COMMIX-PPC Subroutines

\begin{tabular}{|c|c|c|}
\hline Subroutine & $\begin{array}{l}\text { Calling } \\
\text { Subroutine }\end{array}$ & Description \\
\hline MAIN & - & $\begin{array}{l}\text { Writes the title and computes addresses of variables } \\
\text { according to the key variables read from input. }\end{array}$ \\
\hline AMAIN & MAIN & Main calling program. See overall flow chart, Fig. 1. \\
\hline BARIN & INITAL & Reads the boundary and internal cell initialization cards. \\
\hline BCCONT & $\begin{array}{l}\text { INITFL } \\
\text { TIMSTP }\end{array}$ & Updates boundary values on continuative exits. \\
\hline BCFLOT & $\begin{array}{l}\text { INITFL } \\
\text { MOLOOP }\end{array}$ & $\begin{array}{l}\text { Updates boundary velocities on surfaces with transient } \\
\text { flow boundary condition. }\end{array}$ \\
\hline BCFLOW & $\begin{array}{l}\text { INITFL } \\
\text { TIMSTP } \\
\text { MOLOOP }\end{array}$ & Updates boundary velocities during iterations. \\
\hline BCMAS & TIMSTP & Sets continued mass and volume fraction boundary values. \\
\hline BCMASS & $\begin{array}{l}\text { INITFL } \\
\text { TIMSTP }\end{array}$ & $\begin{array}{l}\text { Sets continued mass and volume fraction boundary values } \\
\text { per KMASS. }\end{array}$ \\
\hline BCPRES & $\begin{array}{l}\text { INITFL } \\
\text { TIMSTP }\end{array}$ & $\begin{array}{l}\text { Sets specified pressure values at cells adjacent to } \\
\text { surface having pressure boundary conditions. }\end{array}$ \\
\hline BCTEMP & $\begin{array}{l}\text { INITFL } \\
\text { TIMSTP }\end{array}$ & $\begin{array}{l}\text { Updates boundary values of heat flux, temperature, } \\
\text { density, and enthalpy. }\end{array}$ \\
\hline BCTEMT & $\begin{array}{l}\text { INITFL } \\
\text { ENLOOP }\end{array}$ & $\begin{array}{l}\text { Updates boundary values of temperature, pressure, and } \\
\text { density for constant and transient heat flux boundary } \\
\text { conditions. }\end{array}$ \\
\hline ВСТEM $\varnothing$ & $\begin{array}{l}\text { INITFL } \\
\text { TIMSTP }\end{array}$ & $\begin{array}{l}\text { Computes boundary values of pressure, heat flux, and } \\
\text { density for constant or transient temperature boundary } \\
\text { conditions. }\end{array}$ \\
\hline BCTUBE & TIMSTP & $\begin{array}{l}\text { Updates boundary values of pressure, heat flux, and } \\
\text { density for constant or transient temperature boundary } \\
\text { conditions on tube side. }\end{array}$ \\
\hline BCTURB & TIMSTP & Updates boundary values of turbulence kinetic energy. \\
\hline BOXES & GEOM3D & $\begin{array}{l}\text { Reads surface specification cards input for the box } \\
\text { geometry option. }\end{array}$ \\
\hline
\end{tabular}




\begin{tabular}{|c|c|c|}
\hline Subroutine & $\begin{array}{l}\text { Calling } \\
\text { Subroutine }\end{array}$ & Description \\
\hline CGRAD & SOLVER & $\begin{array}{l}\text { Solves matrix equation by preconditioned conjugate } \\
\text { gradient method. }\end{array}$ \\
\hline CHOP & $\begin{array}{l}\text { OUT2 } \\
\text { OUT1 } \\
\text { OUT11 }\end{array}$ & $\begin{array}{l}\text { Resets the beginning and end row and column markers } \\
\text { to print out only rows and columns containing cell or } \\
\text { boundary values. }\end{array}$ \\
\hline CLEAR & MAIN & $\begin{array}{l}\text { Zeros out the values of all variables between the two } \\
\text { arguments passed. }\end{array}$ \\
\hline DIFFXI & $\begin{array}{l}\text { TDENER } \\
\text { TKENER }\end{array}$ & $\begin{array}{l}\text { Computes diffusion between two adjacent cells for } \mathbf{k} \text { and } \\
\varepsilon \text { equations. }\end{array}$ \\
\hline DMIM & SOLVER & $\begin{array}{l}\text { Solves the matrix equation with the direct matrix } \\
\text { inversion method. }\end{array}$ \\
\hline ECONDX & ENERGI & Computes conduction between two adjacent cells. \\
\hline ENCFY & ENCX2 & $\begin{array}{l}\text { Computes y direction weighting factors for flow- } \\
\text { modulated skew-upwind difference scheme. }\end{array}$ \\
\hline ENCONØ & $\begin{array}{l}\text { TKLOOP } \\
\text { TDLOOP } \\
\text { ENLOOP }\end{array}$ & Computes upwind convective terms. \\
\hline ENCON2 & ENLOOP & $\begin{array}{l}\text { Computes convective flux for flow-modulated skew- } \\
\text { wind difference scheme. }\end{array}$ \\
\hline ENCOR3 & ENCX2 & $\begin{array}{l}\text { Computes corner weighting factors for flow-modulated } \\
\text { skew-upwind difference scheme. }\end{array}$ \\
\hline ENCX2 & ENCON2 & $\begin{array}{l}\text { Computes coefficients due to positive } x \text { direction for } \\
\text { flow-modulated skew-upwind difference scheme. }\end{array}$ \\
\hline ENERGI & ENLOOP & Computes coefficients of the energy equation. \\
\hline ENLOOP & TIMSTP & $\begin{array}{l}\text { Calls required subroutines in sequence for solution of } \\
\text { energy equation. }\end{array}$ \\
\hline ERRCHK & Several & Processes error conditions and prints error messages. \\
\hline ERRMES & Several & Similar to ERRCHK \\
\hline ESORCE & ENLOOP & Computes the source term for energy equation. \\
\hline FILLM & BOXES & Initializes the cell and adjacent cell pointers. \\
\hline FITIT & INITAL & $\begin{array}{l}\text { Computes the coefficients of cubic spline fit for input } \\
\text { transient functions. }\end{array}$ \\
\hline
\end{tabular}




\begin{tabular}{|c|c|c|}
\hline Subroutine & $\begin{array}{c}\text { Calling } \\
\text { Subroutine }\end{array}$ & Description \\
\hline FORCES & $\begin{array}{l}\text { XMOMI } \\
\text { YMOMI } \\
\text { ZMOMI }\end{array}$ & Computes user-imposed drag forces. \\
\hline GDCONV & AMAIN & $\begin{array}{l}\text { Computes DCONV, the convergence criteria based on } \\
\text { maximum velocity in the region. }\end{array}$ \\
\hline GEOM3D & AMAIN & Determines essential geometric information. \\
\hline GEOVAR & INITAL & $\begin{array}{l}\text { Computes cell volumes and surface areas, which remain } \\
\text { constant throughout a calculation. }\end{array}$ \\
\hline GETCON & ENLOOP & Computes effective thermal conductivity. \\
\hline GETEKE & ENLOOP & Computes flow kinetic energy. \\
\hline GETF & several & $\begin{array}{l}\text { Determines the value of transient function } \mathrm{NF} \text { at the } \\
\text { current time. }\end{array}$ \\
\hline GETFLO & $\begin{array}{l}\text { MOLOOP } \\
\text { INITFL }\end{array}$ & Computes mass flow. \\
\hline GETHL & ENLOOP & Computes enthalpy. \\
\hline GETMEM & MAIN & Allocates memory dynamically. \\
\hline GETRHC & $\begin{array}{l}\text { INITFL } \\
\text { TIMSTP }\end{array}$ & Computes boundary density and enthalpy. \\
\hline GETRHM & $\begin{array}{l}\text { INITFL } \\
\text { TIMSTP }\end{array}$ & $\begin{array}{l}\text { Evaluates mixture density and enthalpy, and product of } \\
\text { density and volume fraction at boundary. }\end{array}$ \\
\hline GETVEL & TIMSTP & Adjusts velocity after density change. \\
\hline GETVIS & MOLOOP & Computes effective viscosity for all cells. \\
\hline GLOBAL & OUTPUT & Prints global balances. \\
\hline HSTRUC & $\begin{array}{l}\text { AMAIN } \\
\text { ENLOOP }\end{array}$ & $\begin{array}{l}\text { Computes heat transfer coefficients for thermal- } \\
\text { structure elements. }\end{array}$ \\
\hline HTCOEF & $\begin{array}{l}\text { HSTRUC } \\
\text { BCTEM } \varnothing\end{array}$ & Computes heat transfer coefficient. \\
\hline HTCOND & HTCOEF & Computes heat transfer coefficient for condenser model. \\
\hline HTCTUB & HSATRUC & Computes tube-side heat transfer coefficient. \\
\hline
\end{tabular}




\begin{tabular}{|c|c|c|}
\hline Subroutine & $\begin{array}{l}\text { Calling } \\
\text { Subroutine }\end{array}$ & Description \\
\hline ICTEMP & INITAL & $\begin{array}{l}\text { Initializes default boundary temperature and boundary } \\
\text { velocity (or mass flow). }\end{array}$ \\
\hline ICTUBE & INITAL & Initializes tube-side flow conditions. \\
\hline INFORC & INITAL & $\begin{array}{l}\text { Reads input related to force structure and prints corre- } \\
\text { sponding input summary. }\end{array}$ \\
\hline INITAL & AMAIN & $\begin{array}{l}\text { Calls required subroutines in sequence to initialize } \\
\text { internal-cell and boundary values of all variables. }\end{array}$ \\
\hline INITFL & INITAL & Initializes all fluid variables. \\
\hline INITPR & INITAL & Sets default static pressure. \\
\hline INITUR & INITAL & Initializes turbulence model. \\
\hline INPSTR & INITAL & $\begin{array}{l}\text { Reads thermal structure input data, computes required } \\
\text { geometrical and physical information, and prints } \\
\text { thermal-structure input summary. }\end{array}$ \\
\hline ISURFO & OUTPUT & Prints surface array IVAR for surface number NSUR. \\
\hline MACONØ & MALOOP & $\begin{array}{l}\text { Computes the convective terms for component mass } \\
\text { continuity equation. }\end{array}$ \\
\hline MADIFF & MALOOP & $\begin{array}{l}\text { Computes the diffusion terms for component mass } \\
\text { continuity equation. }\end{array}$ \\
\hline MALOOP & TIMSTP & $\begin{array}{l}\text { Calls required subroutines in sequence to solve } \\
\text { component mass continuity equation. }\end{array}$ \\
\hline MASSCO & MALOOP & $\begin{array}{l}\text { Computes transient and source terms for component } \\
\text { mass continuity equation. }\end{array}$ \\
\hline MOLOOP & TIMSTP & $\begin{array}{l}\text { Calls required subroutines in sequence to solve mass- } \\
\text { momentum equations. }\end{array}$ \\
\hline MOMENI & MOLOOP & $\begin{array}{l}\text { Calculates new-time velocity using new-time values of } \\
\text { pressure. }\end{array}$ \\
\hline NAMELS & $\begin{array}{l}\text { GEO3D } \\
\text { INITAL } \\
\text { ADRIVE }\end{array}$ & Reads NAMELIST information. \\
\hline OUT1 & OUTPUT & Writes a plane of values for cell-centered variables. \\
\hline
\end{tabular}




\begin{tabular}{|c|c|c|}
\hline Subroutine & $\begin{array}{l}\text { Calling } \\
\text { Subroutine }\end{array}$ & Description \\
\hline OUT1I & OUTPUT & $\begin{array}{l}\text { Writes a plane of integer values for cell-centered } \\
\text { varlables. }\end{array}$ \\
\hline OUT2 & OUTPUT & Writes a plane of values for face-centered variables. \\
\hline OUTFIE & OUT 1 & $\begin{array}{l}\text { Sets up two arrays, VAL and MARK, of the size NRMAX } \\
\text { and NCMAX for cell-centered variables. }\end{array}$ \\
\hline OUTFII & OUT1I & $\begin{array}{l}\text { Sets up two arrays, VAL and MARK, of the size NRMAX } \\
\text { and NCMAX for cell-centered variables. }\end{array}$ \\
\hline $\begin{array}{l}\text { OUTIU } \\
\text { OUTJU } \\
\text { OUTKU }\end{array}$ & OUT2 & Similar to OUTFIE for face-centered variables. \\
\hline OUTPTB & OUTTBI & Prints tube-side flow information. \\
\hline OUTPUT & $\begin{array}{l}\text { AMAIN } \\
\text { ERRCHK }\end{array}$ & Prints array information. \\
\hline OUTTBI & OUTPUT & Sets up tube-side variable storage information. \\
\hline PEQN & MOLOOP & Computes coefficients of pressure equations. \\
\hline PLTAPE & $\begin{array}{l}\text { AMAIN } \\
\text { RESTAR }\end{array}$ & Writes plotting information. \\
\hline PRINRC & $\begin{array}{l}\text { OUT1 } \\
\text { OUT2 }\end{array}$ & Writes out the incoming array. \\
\hline PRINRI & OUT11 & Writes out the incoming integer array. \\
\hline PROPF & ROTEMP & Computes properties. \\
\hline PSTRUC & OUTPUT & $\begin{array}{l}\text { Prints temperature fields and heat transfer information } \\
\text { relating to thermal structures. }\end{array}$ \\
\hline QSTRUC & $\begin{array}{l}\text { AMAIN } \\
\text { ESORCE }\end{array}$ & $\begin{array}{l}\text { Solves heat conduction equations and computes effective } \\
\text { heat source from thermal structures to the coolant. }\end{array}$ \\
\hline REDEF & BARIN & $\begin{array}{l}\text { Redefines porosities and permeabilities that are } \mathbf{R} \\
\text { dependent for cylindrical geometry. }\end{array}$ \\
\hline RESTAR & $\begin{array}{l}\text { INITAL } \\
\text { AMAIN }\end{array}$ & Writes or reads a restart dataset. \\
\hline ROTEMP & $\begin{array}{l}\text { TIMSTP } \\
\text { INITFL }\end{array}$ & $\begin{array}{l}\text { Determines the state of each cell by updating the } \\
\text { properties. }\end{array}$ \\
\hline
\end{tabular}




\begin{tabular}{|c|c|c|}
\hline Subroutine & $\begin{array}{l}\text { Calling } \\
\text { Subroutine }\end{array}$ & Description \\
\hline RSURFO & OUTPUT & Prints a specified surface array. \\
\hline RUNID & $\begin{array}{l}\text { MAIN } \\
\text { GLOBAL } \\
\text { PLTAPE }\end{array}$ & Returns time and version information. \\
\hline SETDT & $\begin{array}{l}\text { AMAIN } \\
\text { GDCONV }\end{array}$ & Sets a nonzero time-step size $(0.001)$ \\
\hline SETRL & BARIN & Performs boundary value initialization. \\
\hline SETRM & BARIN & Performs interior-cell value initialization. \\
\hline SHOME & FILLM & $\begin{array}{l}\text { Prints graphical representation of the boundary surface } \\
\text { identification cards. }\end{array}$ \\
\hline SOLVER & $\begin{array}{l}\text { MALOOP } \\
\text { MOLOOP } \\
\text { TKLOOP } \\
\text { TDLOOP } \\
\text { ENLOOP }\end{array}$ & $\begin{array}{l}\text { Calls one of the three matrix solvers to solve the } \\
\text { discretized pressure equation and the scalar transport } \\
\text { equations. }\end{array}$ \\
\hline SOLVIT & SOLVER & Solves the matrix equation by the SOR method. \\
\hline STOSUM & ADRIVE & $\begin{array}{l}\text { Computes the lengths of varlables and prints storage } \\
\text { summaries. }\end{array}$ \\
\hline SUMRE 1 & GLOBAL & Reads and stores a set of input. \\
\hline SUMRE2 & AMAIN & Writes the control variable summary. \\
\hline TBINPR & INITAL & $\begin{array}{l}\text { Reads the tube-side input to set up the storage for tube- } \\
\text { side varlables. }\end{array}$ \\
\hline TBSCAN & MAIN & $\begin{array}{l}\text { Scans tube-side input to determine amount of storage } \\
\text { needed to run a condenser problem. }\end{array}$ \\
\hline TBSIDE & TIMSTP & $\begin{array}{l}\text { Solves one-dimensional Navier-Stoke's equation for tube- } \\
\text { side flow. }\end{array}$ \\
\hline TDENER & TDLOOP & $\begin{array}{l}\text { Computes coefficients of the } \varepsilon \text { (dissipation of turbulence } \\
\text { kinetic energy) equation. }\end{array}$ \\
\hline TDLOOP & TIMSTP & $\begin{array}{l}\text { Computes the dissipation of turbulence kinetic energy } \varepsilon \\
\text { for the two-equation models. }\end{array}$ \\
\hline TDMA & TBSIDE & Tri-diagonal matrix algorithm. \\
\hline
\end{tabular}


Calling

\begin{tabular}{|c|c|c|}
\hline Subroutine & Subroutine & Description \\
\hline TIMSTP & AMAIN & $\begin{array}{l}\text { Calls required subroutines in sequence to bring variable } \\
\text { values from time } t \text { to time } t+\Delta t \text {. }\end{array}$ \\
\hline TKENER & TKLOOP & $\begin{array}{l}\text { Calculates the coefficients of the turbulence kinetic } \\
\text { energy equation. }\end{array}$ \\
\hline TKGRAD & TKSORC & $\begin{array}{l}\text { Computes gradients used in the source terms in the } \\
\text { turbulence equations. }\end{array}$ \\
\hline TKLOOP & TIMSTP & Computes turbulence kinetic energy $\mathbf{k}$. \\
\hline TKSORC & TKLOOP & $\begin{array}{l}\text { Computes the source terms in the turbulence kinetic } \\
\text { energy (k) equation. }\end{array}$ \\
\hline TSAREA & INPSTR & $\begin{array}{l}\text { Computes the total heat transfer area between thernal } \\
\text { structures and fluids. }\end{array}$ \\
\hline TSCAN & MAIN & $\begin{array}{l}\text { Scans the thermal-structure input to determine the } \\
\text { amount of storage needed to run a problem. }\end{array}$ \\
\hline TSQ & GLOBAL & Computes the total heat for each thermal structure. \\
\hline TSTRUC & $\begin{array}{l}\text { AMAIN } \\
\text { TIMSTP }\end{array}$ & $\begin{array}{l}\text { Solves the heat conduction equation and computes } \\
\text { thermal-structure temperature. TTS. }\end{array}$ \\
\hline TURV 12 & $\begin{array}{l}\text { INITFL } \\
\text { TIMSTP }\end{array}$ & $\begin{array}{l}\text { Computes turbulent viscosity and thermal conductivity } \\
\text { for the } k-\varepsilon \text { two-equation turbulence model. }\end{array}$ \\
\hline UPDATB & $\begin{array}{l}\text { INITAL } \\
\text { TIMSTP }\end{array}$ & $\begin{array}{l}\text { Restores previous time-step values to current time-step } \\
\text { values on the tube side. }\end{array}$ \\
\hline UPDATE & $\begin{array}{l}\text { INITFL } \\
\text { TIMSTP }\end{array}$ & $\begin{array}{l}\text { Restores the previous time-step values to the current } \\
\text { time-step values. }\end{array}$ \\
\hline VELCEN & $\begin{array}{l}\text { TKSORC } \\
\text { TKGRAD }\end{array}$ & Computes cell-centered velocity. \\
\hline VSTARØ & FORCES & Computes local critical velocity for superheated steam. \\
\hline WLFNCV & ZMOMX & $\begin{array}{l}\text { Modifies the wall shear stress to account for frictional } \\
\text { force. }\end{array}$ \\
\hline XMOMI & MOLOOP & Computes coefficients of $\mathrm{x}$-momentum equations. \\
\hline YMOMI & MOLOOP & Computes coefficients of $y$-momentum equations. \\
\hline ZMOMI & MOLOOP & Computes coefficients of $\mathrm{z}$-momentum equations. \\
\hline
\end{tabular}


Calling

Subroutine Subroutine

Description

ZMOMX

XMOMI

YMOMI

ZMOMI

ZMOMZ

XMOMI

YMOMI

ZMOMI

ZSUM

INITAL momentum equation. momentum equation.

Prints the property summaries.

Computes the $z-x$ convective and viscous terms of the

Computes the $\mathrm{z}-\mathrm{z}$ convective and viscous terms of the 


\section{Appendix C. Flow-Resistance Correlations}

\section{C.1 Introduction}

This appendix, which contains a conventent collection of flow-resistance correlations that are commonly needed by COMMIX users, is written with hope that it will save the user from the need to search a vast literature. We also hope that this appendix will serve as a starting reference for new users.

We caution here that the correlations presented are not necessarily the only and final relations. We welcome feedback and comments from all users so that we can add other correlations, update the existing relations, and improve the information presented here.

\section{C.2 Crossfiow Over Tubes}

The pressure drop for flow over a bank of tubes can be estimated with the equation

$$
\Delta P=\frac{2 f^{\prime} G_{\max }^{2} N}{\rho}\left(\frac{\mu_{w}}{\mu_{b}}\right)^{0.14}
$$

where

$$
\begin{aligned}
& \Delta \mathbf{P}=\text { pressure drop. } \mathrm{Pa} \text {, } \\
& \mathrm{G}_{\max }=\text { mass velocity at minimum flow area, } \mathrm{kg} / \mathrm{m}^{2} \bullet \mathrm{sec} \text {, } \\
& \rho=\text { density of fluid. } \mathrm{kg} / \mathrm{m}^{3} \text {. } \\
& \mathbf{N}=\text { total number of transverse flows in the main flow direction, } \\
& \mu_{w}=\text { viscosity evaluated at wall temperature, } \mathrm{kg} / \mathrm{m} \bullet \mathrm{s} \text {, } \\
& \mu_{\mathrm{b}}=\text { viscosity evaluated at bulk temperature, } \mathrm{kg} / \mathrm{m} \cdot \mathrm{s} \text {, and } \\
& f=\text { an empirical friction factor given by JakobC. } 1 \text { as } \\
& f^{\prime}=\left[0.25+\frac{0.118}{\left(\frac{S_{T}-D_{0}}{D_{0}}\right)^{1.08}}\right]\left(\frac{G_{\max } D_{0}}{\mu_{b}}\right)^{-0.16}
\end{aligned}
$$

for staggered tube arrangement (Fig. C.1), and

$$
f^{\prime}=\left[0.044+\frac{0.08 S_{L} / D_{0}}{\left(\frac{S_{T}-D_{0}}{D_{0}}\right)^{0.43+1.13 D_{0} / S_{L}}}\right]\left(\frac{G_{\max } D_{0}}{\mu_{b}}\right)^{-0.15}
$$

for in-line tube arrangements (Fig. C.2). 


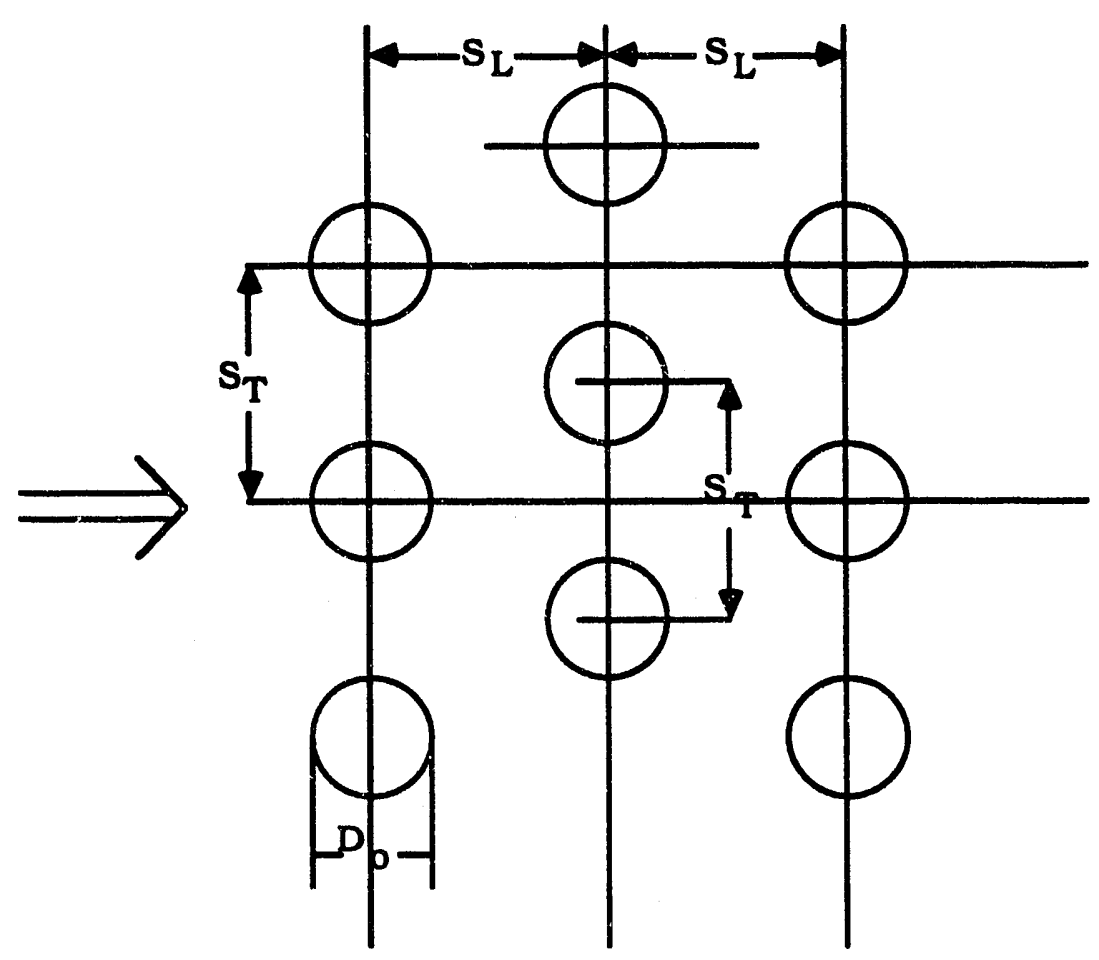

Fig. C.1. Nomenclature for a staggered tube arrangement

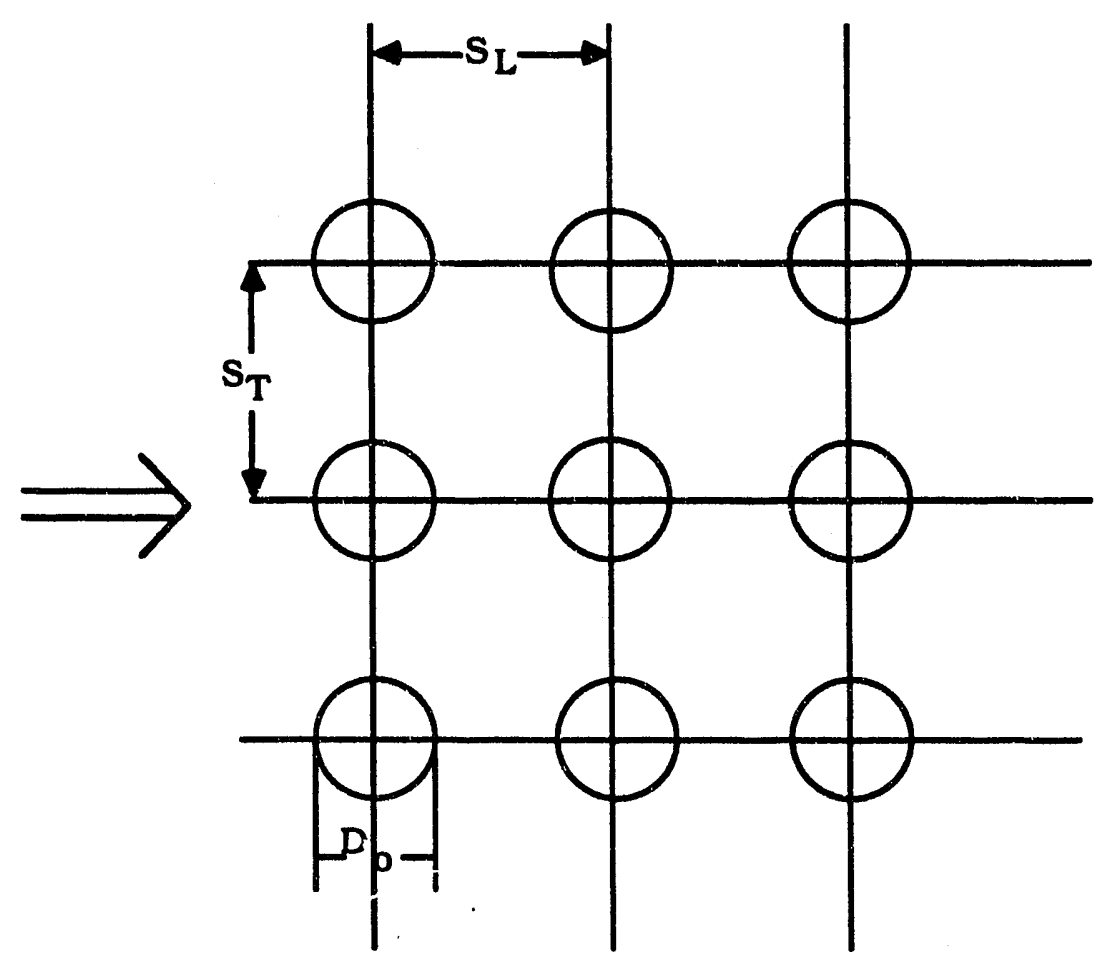

Fig. C2. Nomenclature for in-line tube arrangement 
In Eqs. C.2 and C.3,

$D_{0}=$ outside diameter of tube, $m$.

$\mathrm{S}_{\mathrm{T}}=$ transverse pitch, $\mathrm{m}$, and

$\mathrm{S}_{\mathrm{L}}=$ longitudinal pitch, $\mathrm{m}$.

\section{C.3 Sudden Enlargement and Contraction}

Pressure loss due to abrupt change in a flow area is generally expressed in terms of the loss coefficient $\mathrm{K}$.

$$
\begin{aligned}
\Delta p & =K_{1} \frac{1}{2} \rho v_{1}^{2} \\
& =K_{2} \frac{1}{2} \rho v_{2}^{2},
\end{aligned}
$$

where $K_{1}$ and $K_{2}$ are the pressure loss coefficients, and $v_{1}$ and $v_{2}$ refer to the velocity in smaller and larger cross sec ions, respectively.

For abrupt expansion, the loss coefficient $K$ is given by the expression c.2-C.3

$$
K_{1}=\left(1-\frac{A_{1}}{A_{2}}\right)^{2} \text {. }
$$

where $A_{1}$ and $A_{2}$ are the small and large areas, as shown in Fig. C.3. If the abrupt expansion is modeled as shown in Fig. C.4, the reference velocity used in COMMLX to evaluate pressure drop corresponds to the velocity in the larger cross section. Therefore, we must use Eq. C.5 with the loss coefficient $K_{2}$. given by

$$
K_{2}=\left(\frac{A_{2}}{A_{1}}-1\right)^{2}
$$

For abrupt contraction, as shown in Figs. C.5 and C.6, the values of the loss coefficient are presented in Table C.1, which is taken from Ref. C.4.

\section{C.4 Venturi, Nozzle, and Orifice}

\section{C.4.1 Venturi}

For flow through venturi, the pressure loss can be determined from

$$
\begin{aligned}
\Delta \mathrm{p}_{\text {low }} & =\mathrm{K}_{1} \frac{1}{2} \rho v_{1}^{2} \\
& =\mathrm{K}_{2} \frac{1}{2} \rho v_{2}^{2} .
\end{aligned}
$$

where $K_{1}$ and $K_{2}$ are the loss coefficients of kinetic energy in the small and large cross sections, respectively.

The loss coefficients for venturi, shown in Figs. C.7 and C.8, are given respectively by 


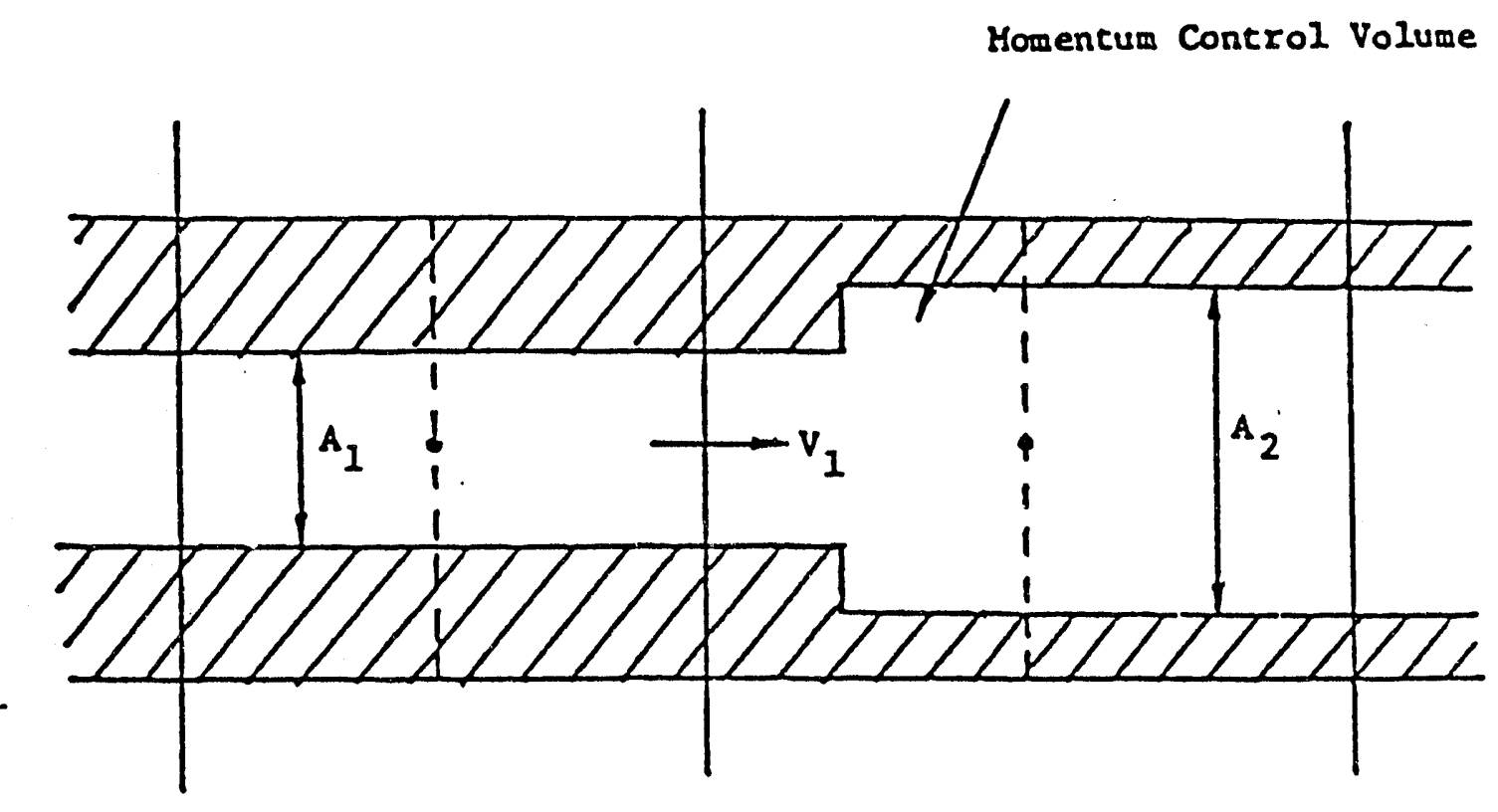

Fig. C.3. Sudden enlargement with reference velocity $V_{1}$

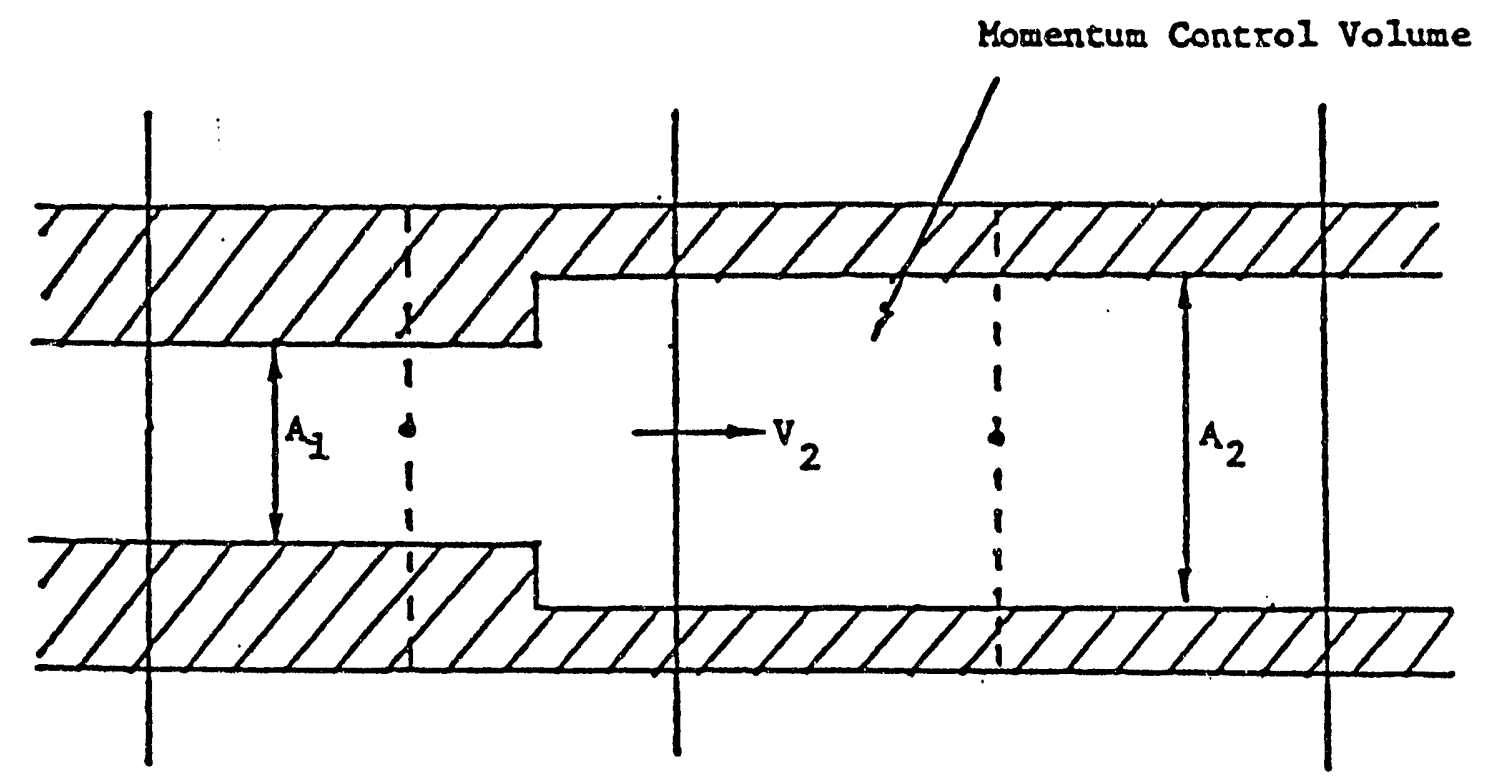

Fig. C.4. Sudden enlargement with reference velocity $V_{2}$ 


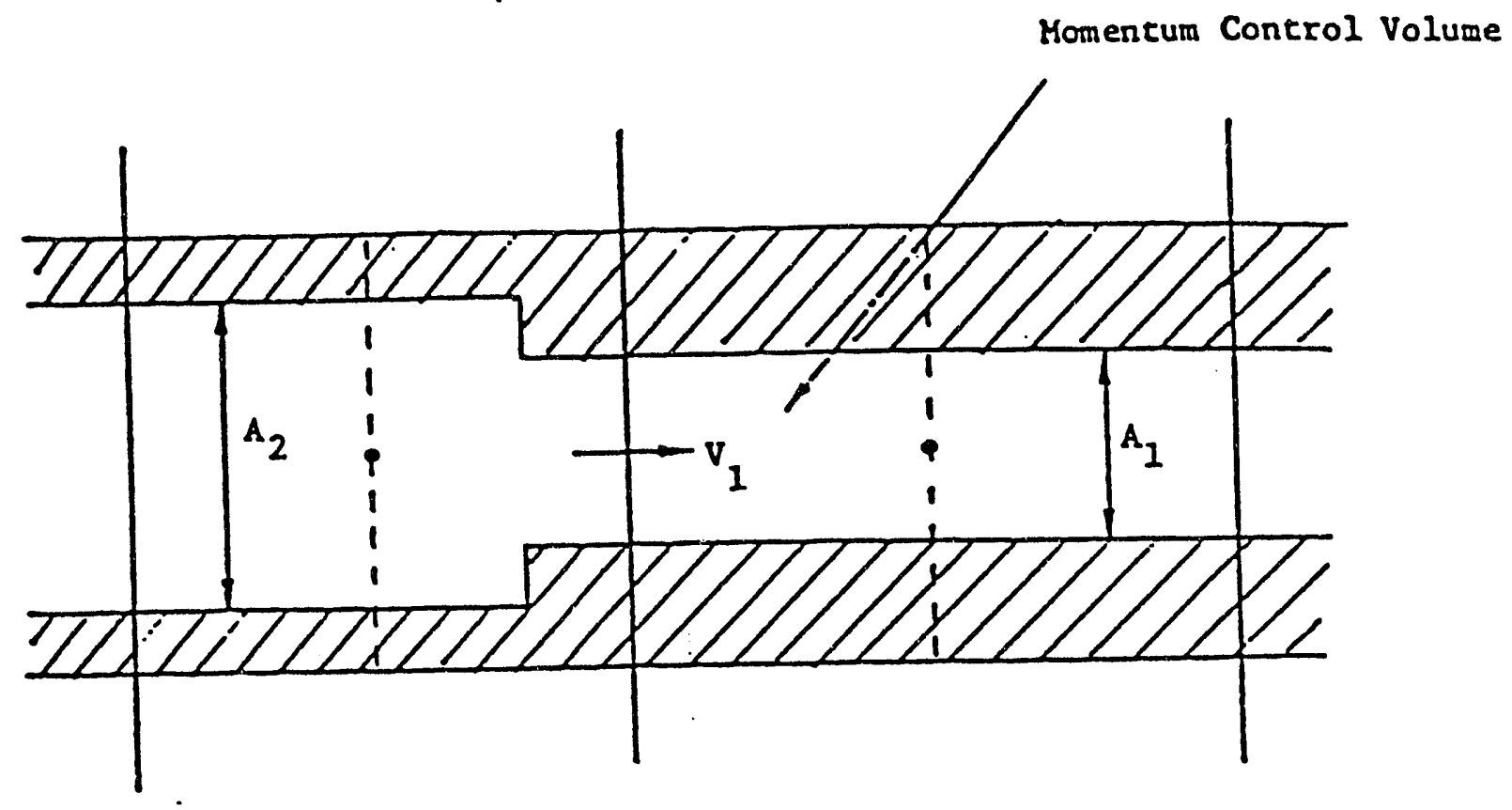

Fig. C.5. Sudden contraction with reference velocity $V_{1}$

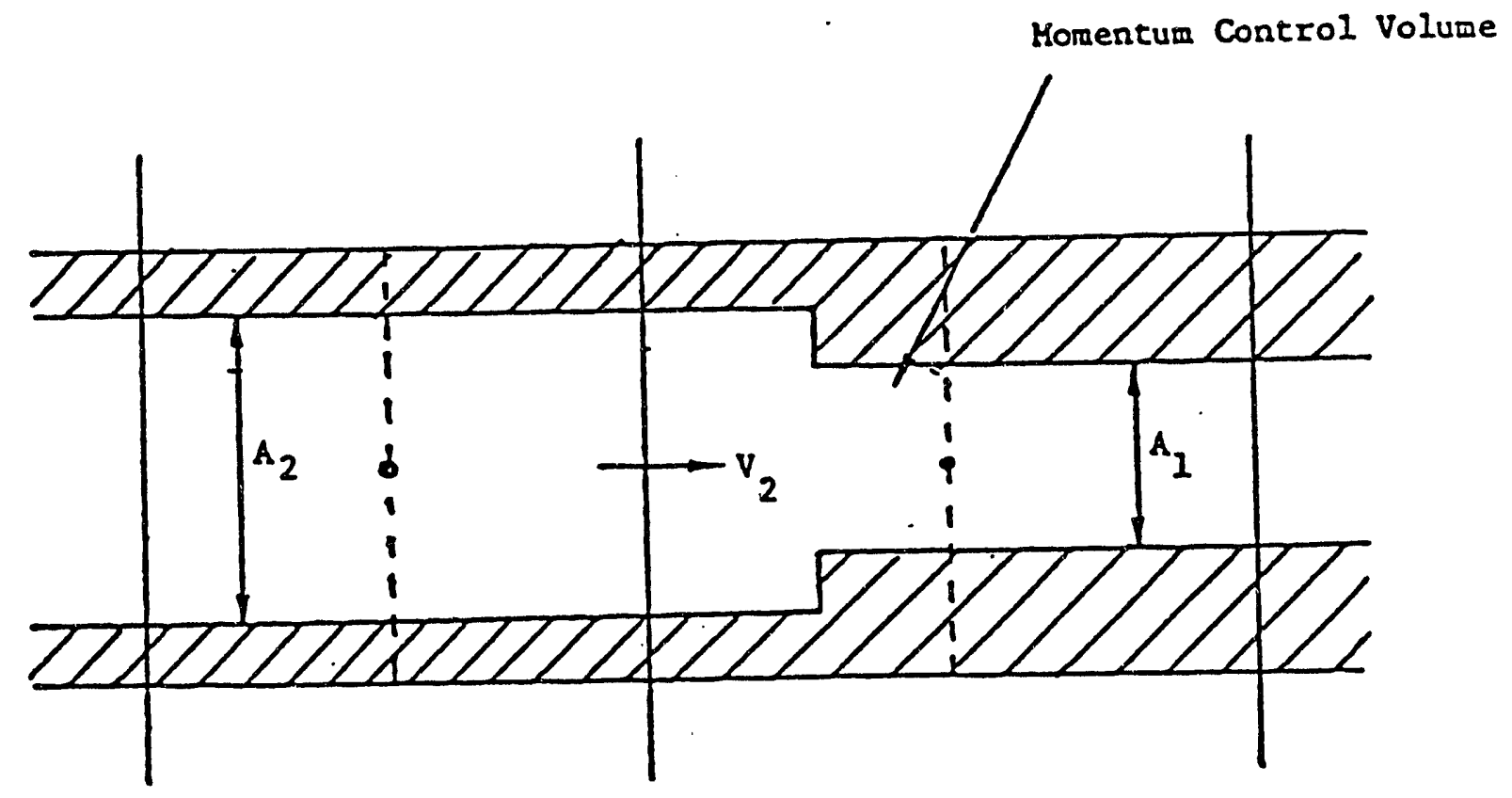

Fig. C.6. Sudden contraction with reference velocity $V_{2}$ 
Table C.1. Loss coefficients for sudden contractiona

\begin{tabular}{llll}
\hline $\mathrm{A}_{1} / \mathrm{A}_{2}$ & $\mathrm{C}_{\mathrm{c}}$ & $\mathrm{K}_{1}$ & $\mathrm{~K}_{2}$ \\
\hline 0.1 & 0.624 & 0.363 & 36.375 \\
0.2 & 0.632 & 0.339 & 8.475 \\
0.3 & 0.643 & 0.308 & 3.422 \\
0.4 & 0.659 & 0.268 & 1.675 \\
0.5 & 0.681 & 0.219 & 0.876 \\
0.6 & 0.712 & 0.164 & 0.456 \\
0.7 & 0.755 & 0.105 & 0.214 \\
0.8 & 0.813 & 0.053 & 0.083 \\
0.9 & 0.892 & 0.015 & 0.019 \\
1.0 & 1.0 & 0 & 0 \\
\hline
\end{tabular}

aSource: Ref. C.4

$$
K_{1}=\left[1-\left(\frac{A_{1}}{A_{2}}\right)^{2}\right]\left(\frac{1}{C_{V}^{2}}-1\right)
$$

and

$$
K_{2}=\left[\left(\frac{A_{2}}{A_{1}}\right)^{2}-1\right]\left(\frac{1}{C_{V}^{2}}-1\right),
$$

where the velocity coefficient $c v$ is a function of Reynolds number $\left(\rho v_{1} D_{1} / \mu\right)$, as listed in Table C.2. The Reynolds number is based on the velocity and diameter of the small cross section.

\section{C.4.2 Nozzle and Oriflce}

For flow through a nozzle and orifice, shown in Figs. C.9 and C.10, the pressure loss is determined from

$$
\Delta \mathrm{p}=\mathrm{K} \frac{1}{2} \rho \mathrm{v}^{2} .
$$

where

$$
K=\frac{1}{C^{2}}\left(\frac{A_{2}}{A_{1}}\right)^{2}
$$




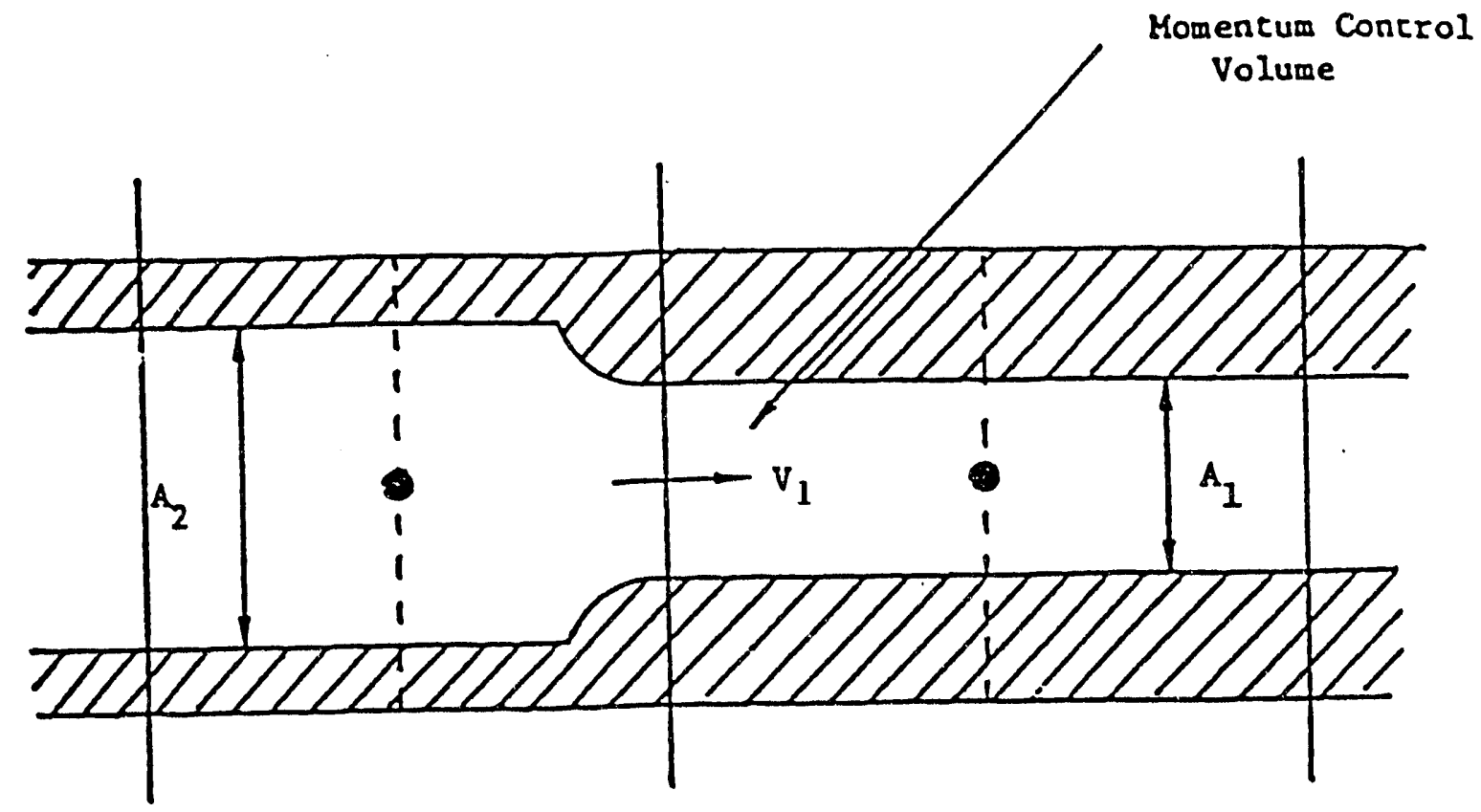

Fig. C.7. Venturi in a momentum control volume, where the loss coefficient is defined by Eq. C.10

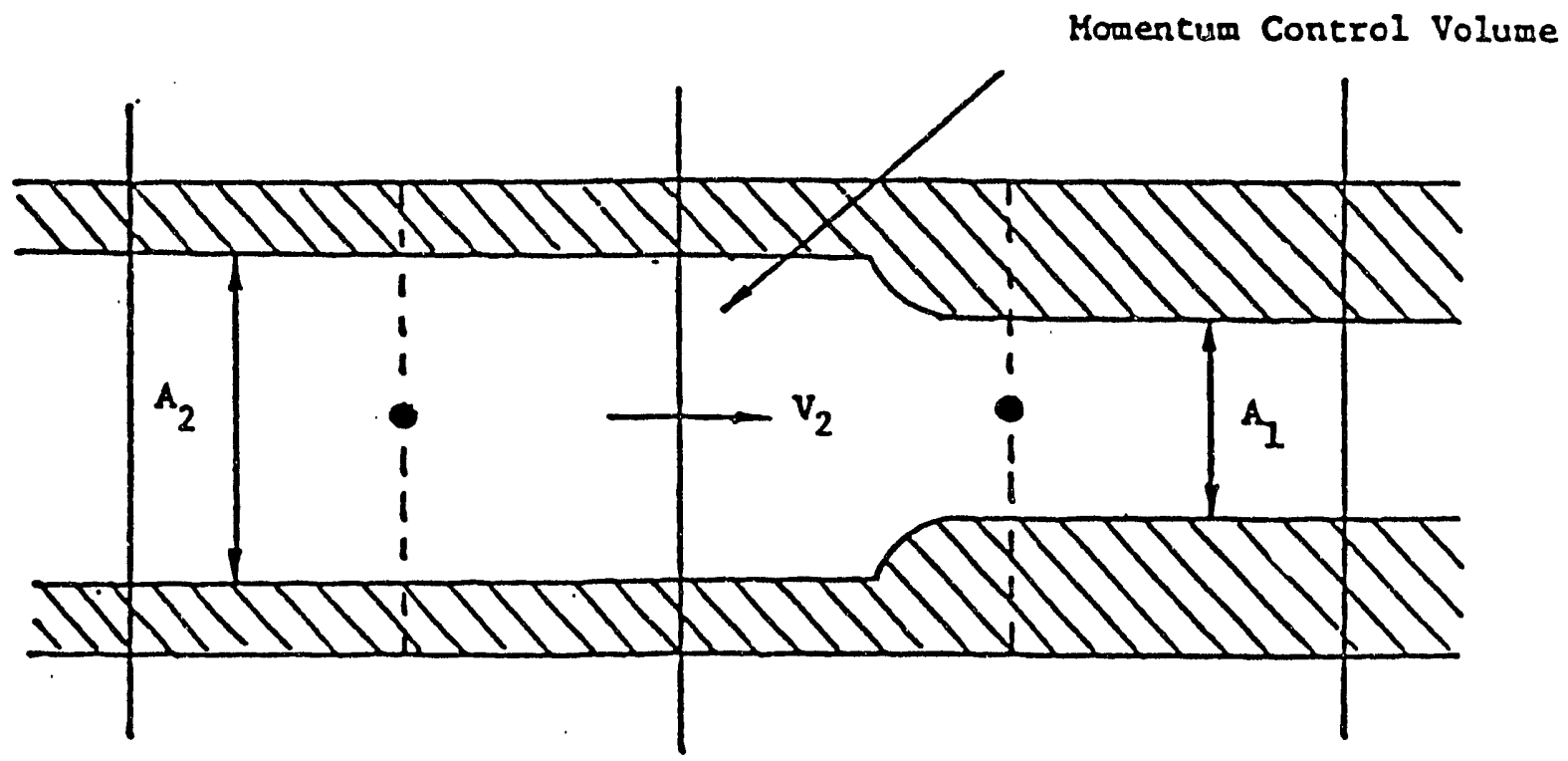

Fig. C.8. Venturi in a momentum control volume, where the loss coefficient is defined by Eq. C.11 
Table C.2. Velocity coefficients $c_{v}$ for ventur ${ }^{a}$

\begin{tabular}{cc}
\hline $\log _{10} R e$ & $c_{v}$ \\
\hline 3.2 & 0.9 \\
3.5 & 0.925 \\
4.0 & 0.950 \\
4.5 & 0.966 \\
5.0 & 0.977 \\
5.5 & 0.985 \\
6.0 & 0.989 \\
6.5 & 0.992 \\
\hline
\end{tabular}

aSource: Ref. C5

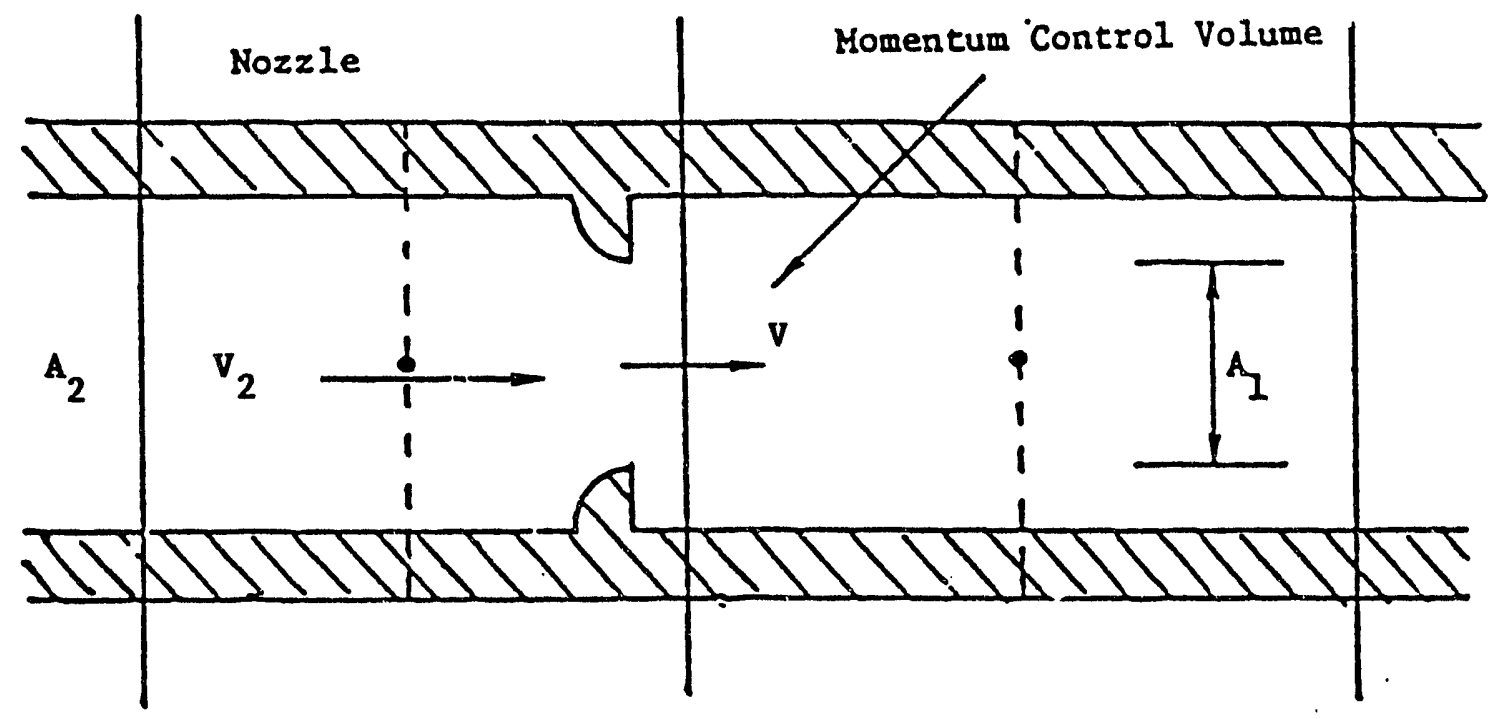

Fig. C.9. Nozzle in a momentum control volume 


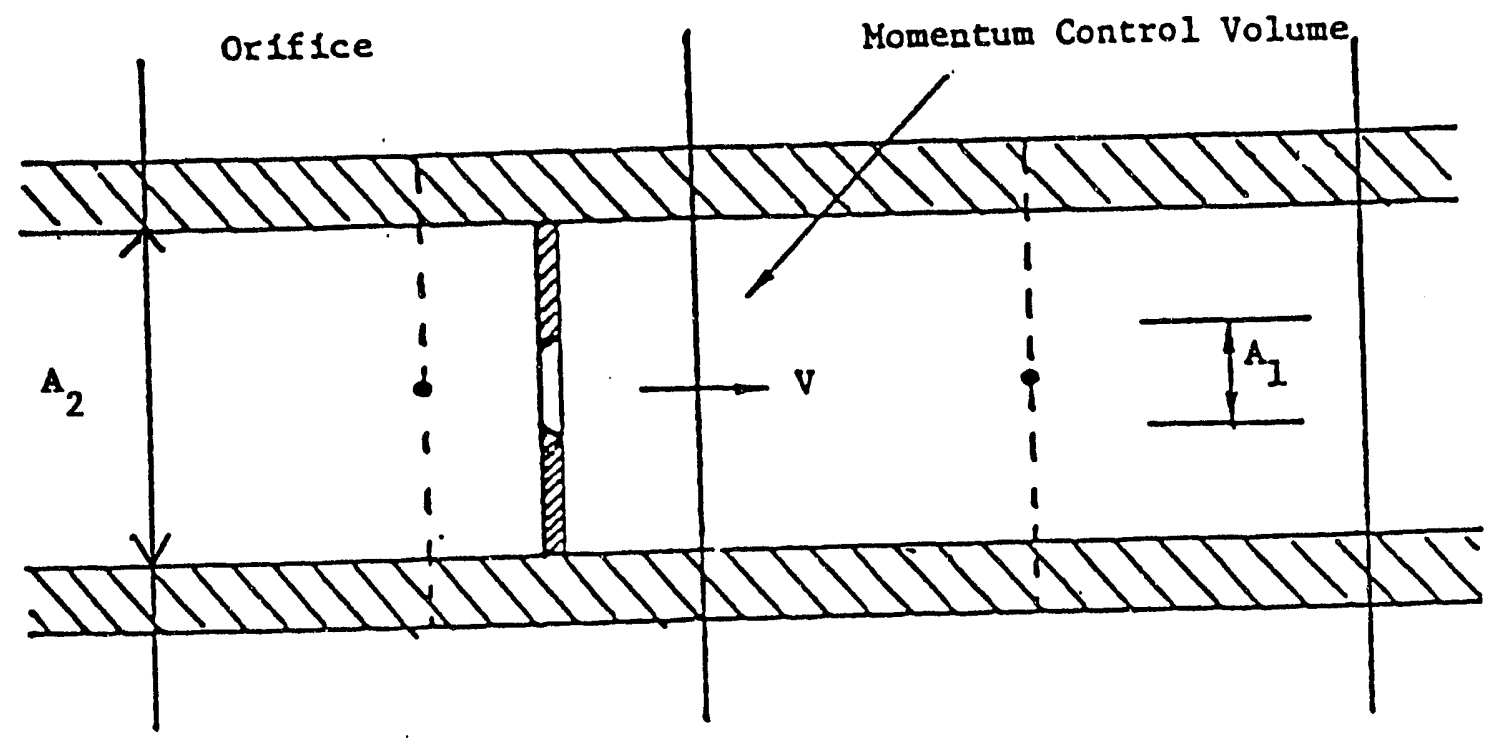

Fig. C.10. Orifice in a momentum control volume

Here, the discharge coefficient $C$ is a function of the area ratio and Reynolds number. The Verein Deutsche Ingeniere (VDI) has published curves for $\mathbf{C}$ for the German standard nozzles and orifices. They are shown in Figs. C.11 and C.12. Note that the Reynolds numbers in both figures are based on the diameter and undisturbed velocity upstream of the nozzle or orifice.

\section{C.5 Submerged Body}

The pressure loss due to a submerged object, shown in Fig. C.13, is given by

$$
\Delta p=C_{D} \frac{A_{p}}{A_{0}}\left(\frac{1}{2} \rho v^{2}\right)
$$

Here, $A_{p}$ is the projected area of a submerged object in a plane normal to the flow direction, $A_{0}$ is the flow area, and $C_{D}$, the drag coefficient, is a function of Reynolds number based on the undisturbed velocity $v_{\infty}$ and the object diameter $D$. Figure C. 14 gives the variation of $C_{D}$ with Reynolds number for several common shapes. 


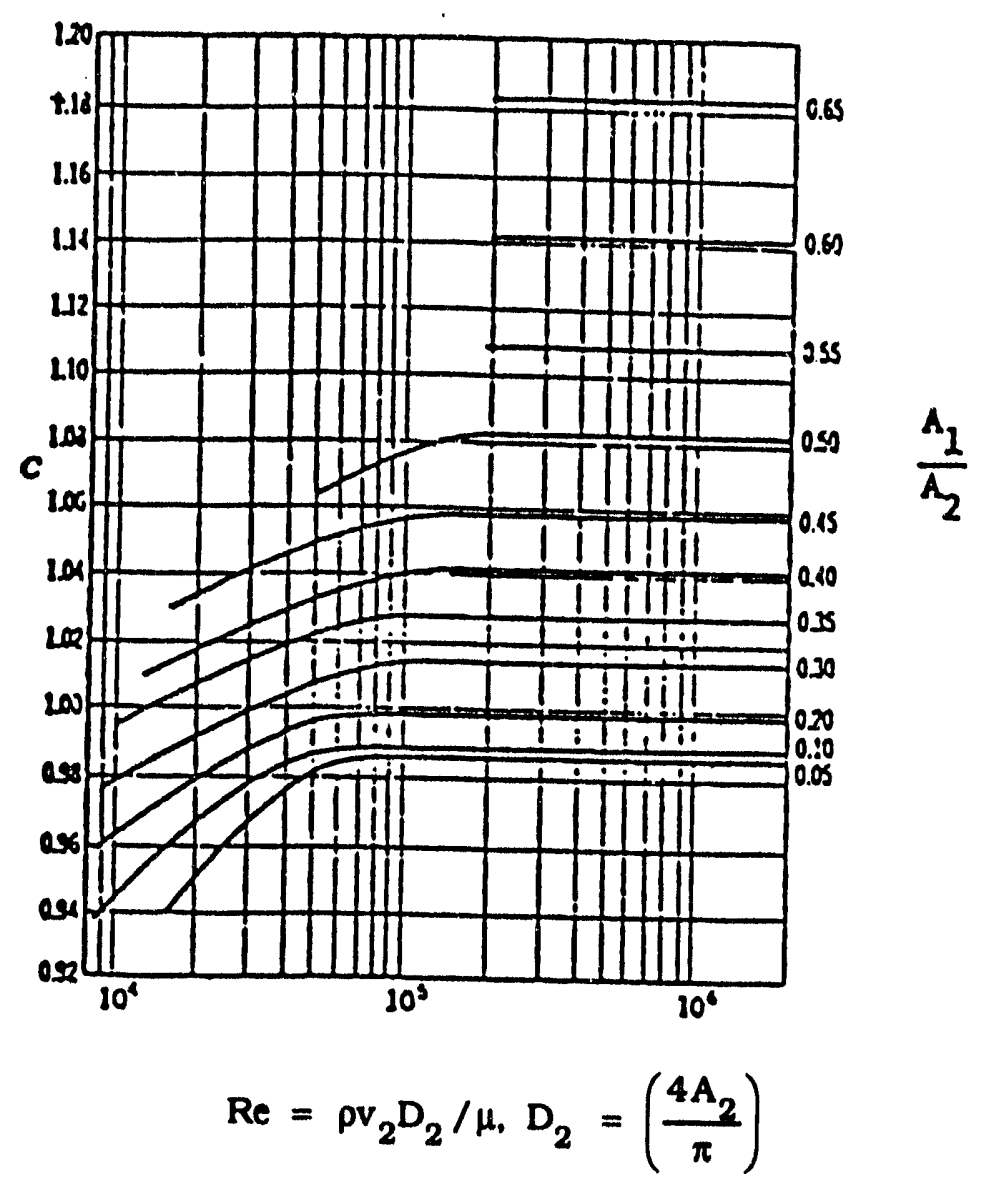

Fig. C.11. Discharge coefficient for VDI nozzle (Source: Ref. C.4)

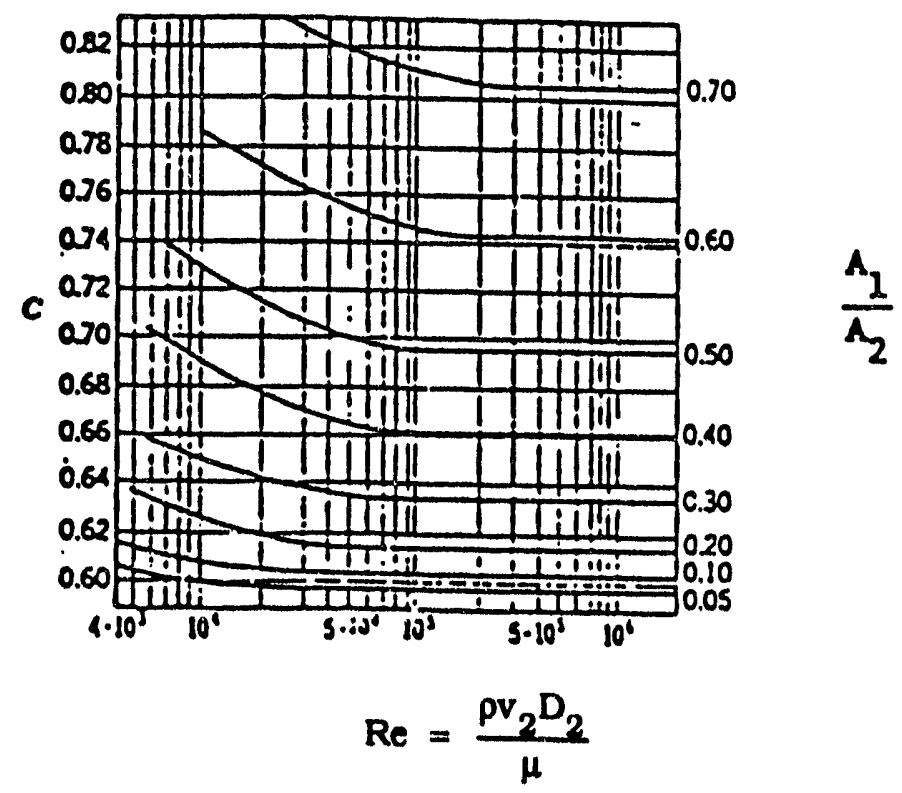

Fig. C.12. Discharge coefficient for VDI orifice (Source: Ref. C.4) 
Momentum Control Volume

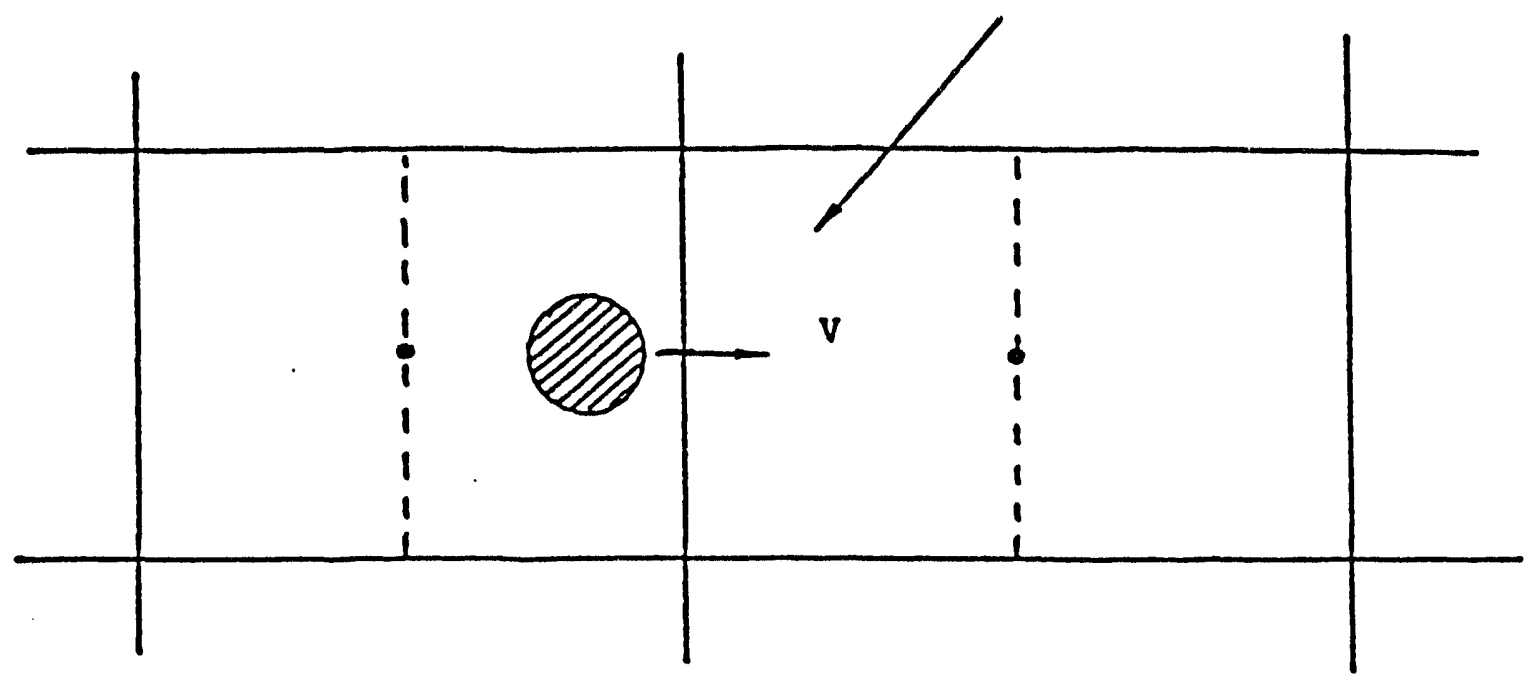

Fig. C.13. Submerged object in a momentum control volume

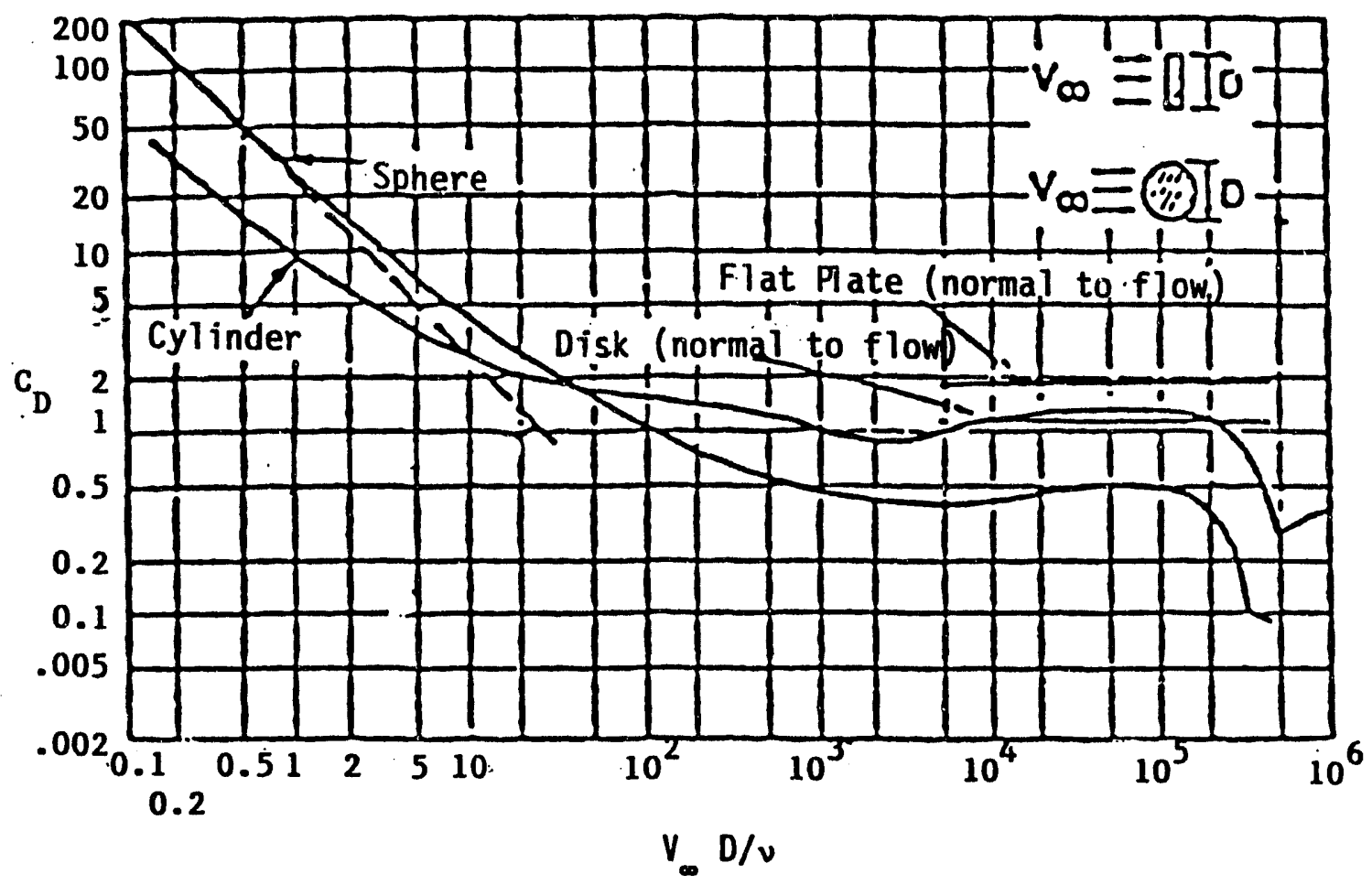

Fig. C.14. Drag coefficient for several simple shapes (Source: Ref. C.4) 


\section{C.6 Straight Duct}

For flow through a straight duct. Fig. C.15, the pressure loss is expressed as

$$
\Delta p=\frac{L}{d_{h}}\left(\frac{1}{2} \rho v^{2}\right) f .
$$

Here, $L$ is the length of the control volume, $d_{h}$ is the hydraulic diameter, and the friction factor $f$ is a function of Reynolds number.

- Laminar Flow

For a circular duct, the fully developed friction factor is given by

$$
f=\frac{64}{\operatorname{Re}} \text {. }
$$

The fully developed friction factor for a family of rectangular ducts ranging from a square to flow between two parallel plates is plotted in Fig. C.16.

- Turbulent Flow

For turbulent flow in a straight duct, the friction factor given by Blasius $\mathrm{C.} 7$ is

$$
f=\frac{0.3164}{\operatorname{Re}^{0.25}}
$$

where

$$
\operatorname{Re}=\rho f_{h} / \mu .
$$

The Blasius formula is valid for $\operatorname{Re}<2 \times 10^{5}$.

\section{C.7 Pipe Fittings}

The pressure loss due to pipe fittings is commonly expressed by

$$
\Delta \mathrm{p}=\mathrm{K} \frac{1}{2} \rho v^{2} .
$$

The head loss coefficients $\mathrm{K}$ for typical fittings, published by Crane Company, $\mathrm{C.8}$ are listed in Table C.3.

\section{C.8 Concluding Remarks}

In Appendix $\mathrm{C}$, we have presented a set of pressure loss correlations for the convenience of COMMIX users. For geometries not included here, Refs. C.9 and C.10 may be consulted.

If experimental data are avallable for any special geometry under consideration, it is preferable to use the data rather than the correlations. 


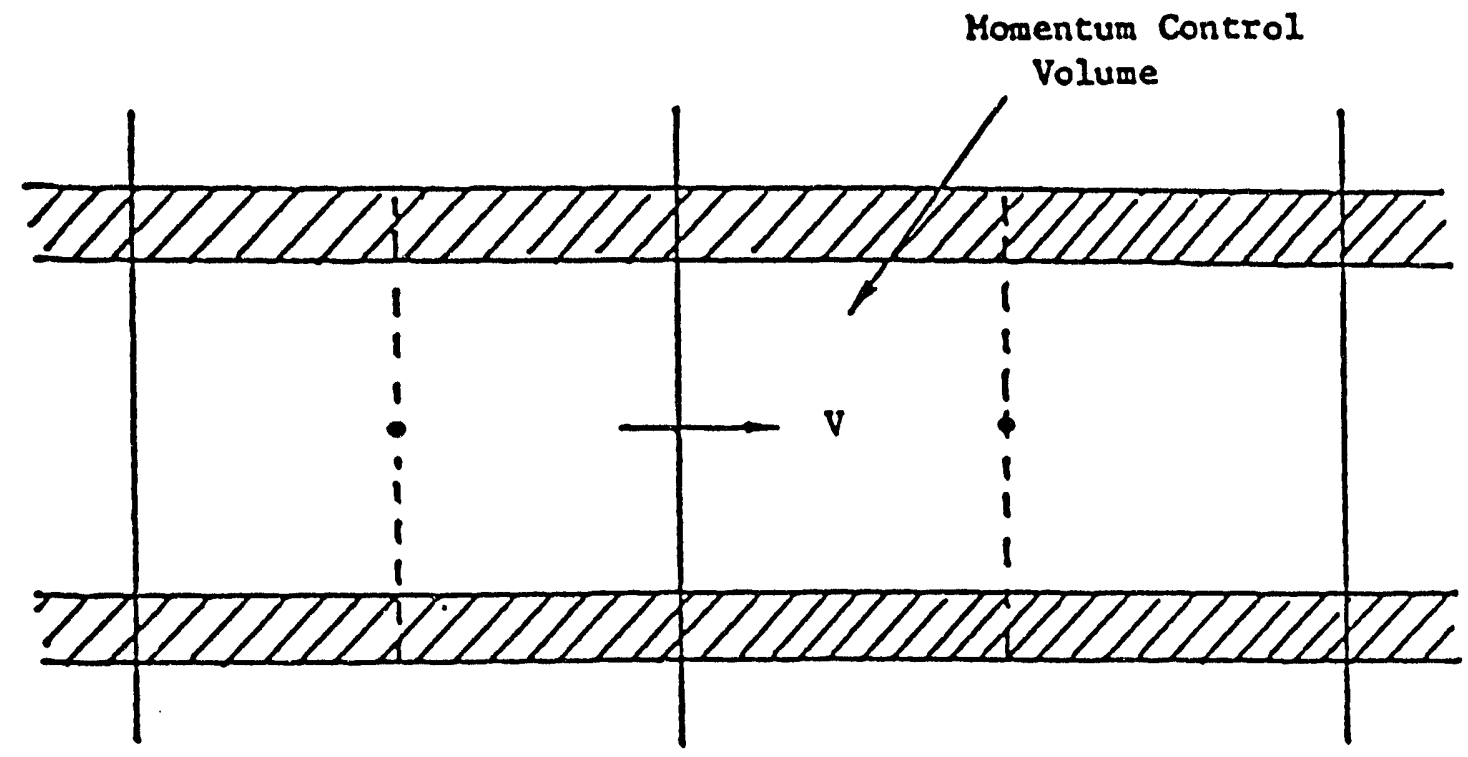

Fig. C.15 Straight duct in a momentum control volume

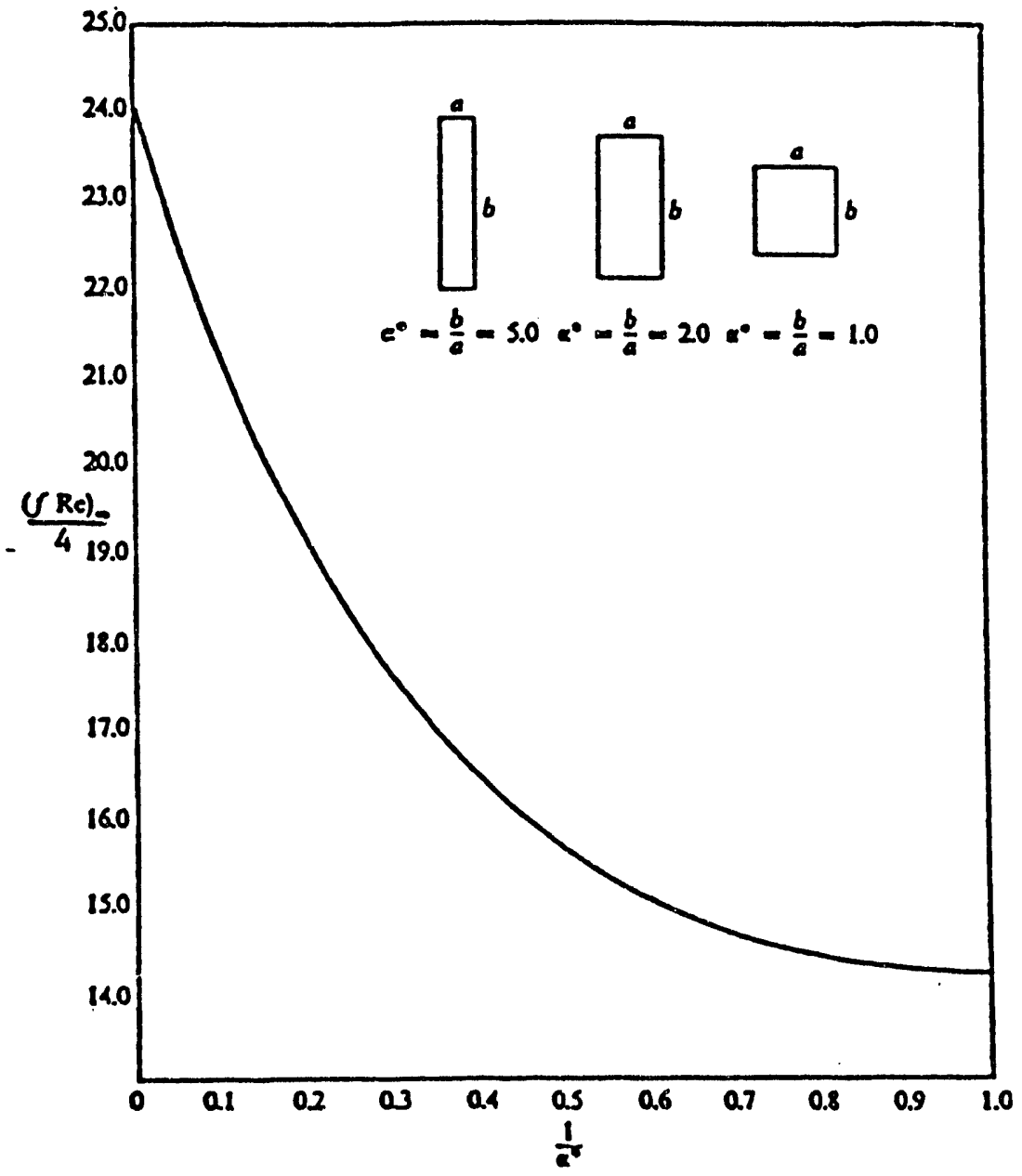

Fig. C.16. Friction coefficients for fully developed laminar flow in rectangular tubes (Source: Ref. C.6) 
Table C.3. Head loss coefficients $\mathrm{K}$ for varlous fittings

\begin{tabular}{lc}
\hline \multicolumn{1}{c}{ Fitting } & $\mathrm{K}$ \\
\hline Giobe valve (fully open) & 10.0 \\
Angle valve (fully open) & 5.0 \\
Swing check valve (fully open) & 2.5 \\
Gate valve (fully open) & 0.19 \\
Close return bend & 2.2 \\
Standard tee & 1.8 \\
Standard elbow & 0.9 \\
Medium sweep elbow & 0.75 \\
Long sweep elbow & 0.60 \\
\hline
\end{tabular}

\section{References for Appendix C}

C.1. M. Jakob. Heat Transfer and Flow Resistance in Cross Flow of Gases over Tube Banks, Trans. ASME, 60, 384 (1938).

C.2 Y. S. Tang, R. D. Coffield, Jr., and R. A. Markley. Thermal Analysis of Liquid Metal Fast Breeder Reactors. Published by American Nuclear Society (1978).

C.3 A. K. Agrawal and M. Khatib-Rahbar, Dynamic Simulation of LMFBR Systems, Atomic Energy Review 18(2):329-552 (1980).

C.4. V. L. Streeter and E. B. Wylie, Fluid Mechanics, McGraw Hill, New York (1975).

C.5. J. C. Hunsaker and B. G. Rightmire, Engineering Applications of Fluid Mechanics, McGraw Hill. New York (1947).

C.6. W. M. Kays, Convective Heat and Mass Transfer, McGraw Hill, New York (1966).

C.7. H. Blasius, Das Ahnlichkeitsgesetz bel Reibungsvorgängen in Flüssigkeiten, Forschg. Arb. Ing.-Wes. No. 134, Berlin (1913).

C.8 Crane Company, Flow of Fluids, Tech. Paper 409, Chicago (May 1942).

C.9. I. E. Idelchik, Handbook of Hydraulic Resistance, Hemisphere Publishing Corp., New York (1986).

C.10. Applied Fluid Dynamics Handbook, Van Nostrand Company, Inc., New York (1984). 


\section{Appendix D. Sample Problem: Steady-State Performance of a Power Plant Condenser in the Presence of Noncondensible Air}

\section{D.1 Purpose}

The purpose of this appendix is to familiarize readers with the input/output structure of the code.

\section{D.2 Problem Description}

The simple problem presented here is a $1 / 2$ shell of a typical nuclear power plant condenser. Figure D. 1 shows the grid structures in the $x, y$, and $z$ directions for the present coarse model. The $J=10$ plane is a symmetrical plane. Figures D.2-D.4 show the grid layout at the $I=4, J=7$, and $J=9$ planes, respectively. A steam/air mixture enters at the condenser top. i.e., $K=2$ plane. It is explicttly assumed here that all the air leaked into the condenser is lumped and accounted for through the condenser inlet boundary condition. The steam from the feed pump turbine exhaust enters into the lower portion of the condenser at cells $(2,9,4)$ and $(2,10,4)$. The noncondensible air, with some steam, is extracted out of the condenser through the air extractor pipes.

All the condenser tubes are appropriately distributed over 38 tube channeis as shown in Fig. D.2. Each tube channel is modeled into 5 nodes along the $x$-direction $(I=2$ to $I=$ 6). The cooling water enters at cell $\mathrm{J}=6$ and exits at cell $\mathrm{I}=2$.

\section{D.3 Input}

\section{D.3.1 Working Fluids}

Shell side: steam and air

Tube side: sea water

\section{D.3.2 Condenser Tubes}

Number of tubes: 10.734

Tube material: Titanium

Outside diameter: $0.02540 \mathrm{~m}$ (1.0 in.)

Inside diameter: $0.02398 \mathrm{~m}(0.944 \mathrm{in}$.

Tube arrangement: staggered with pitch $=0.03175 \mathrm{~m}(1.25 \mathrm{in}$. 


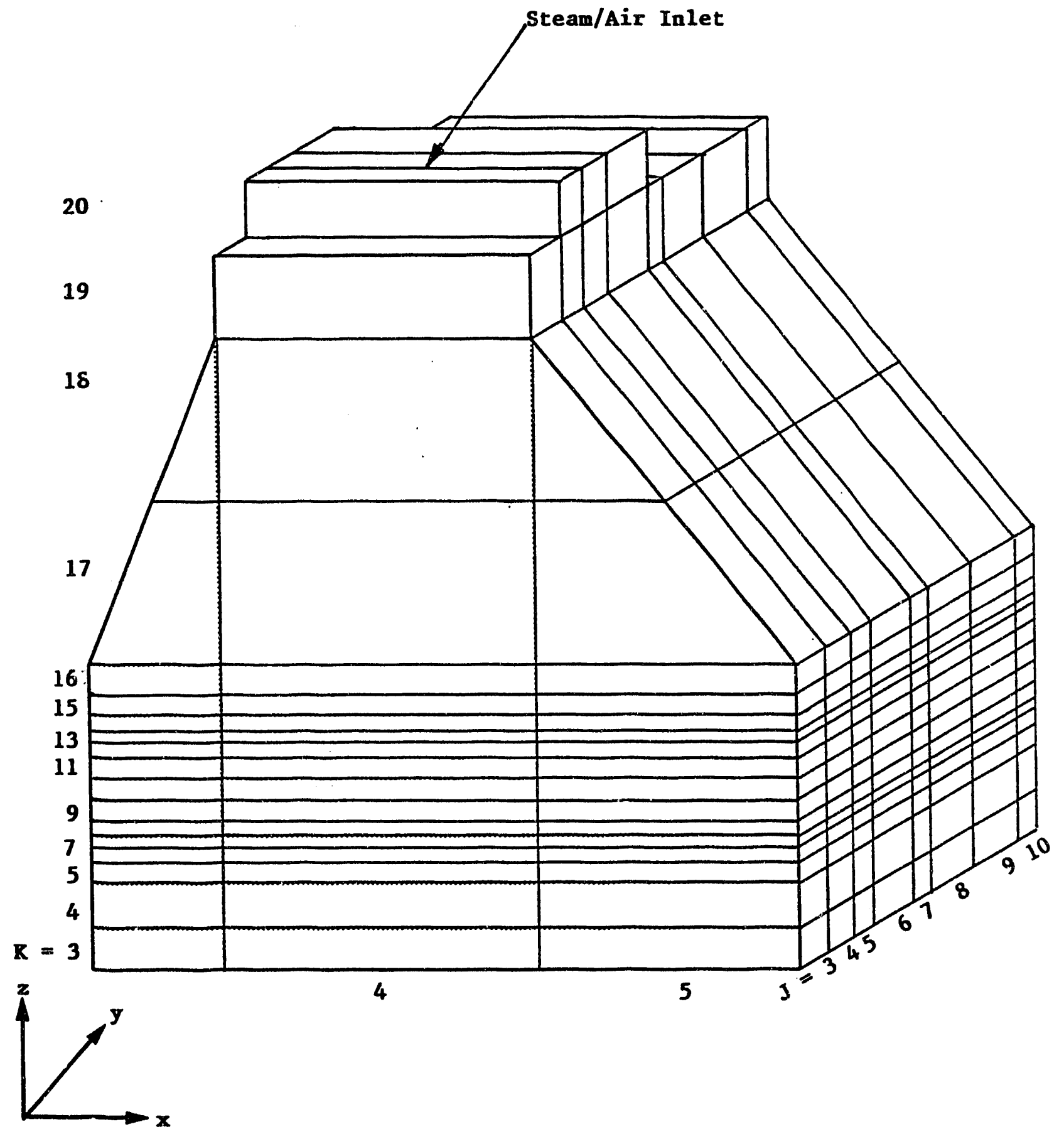

Fig. D.1. Grid structure of the sample problem 


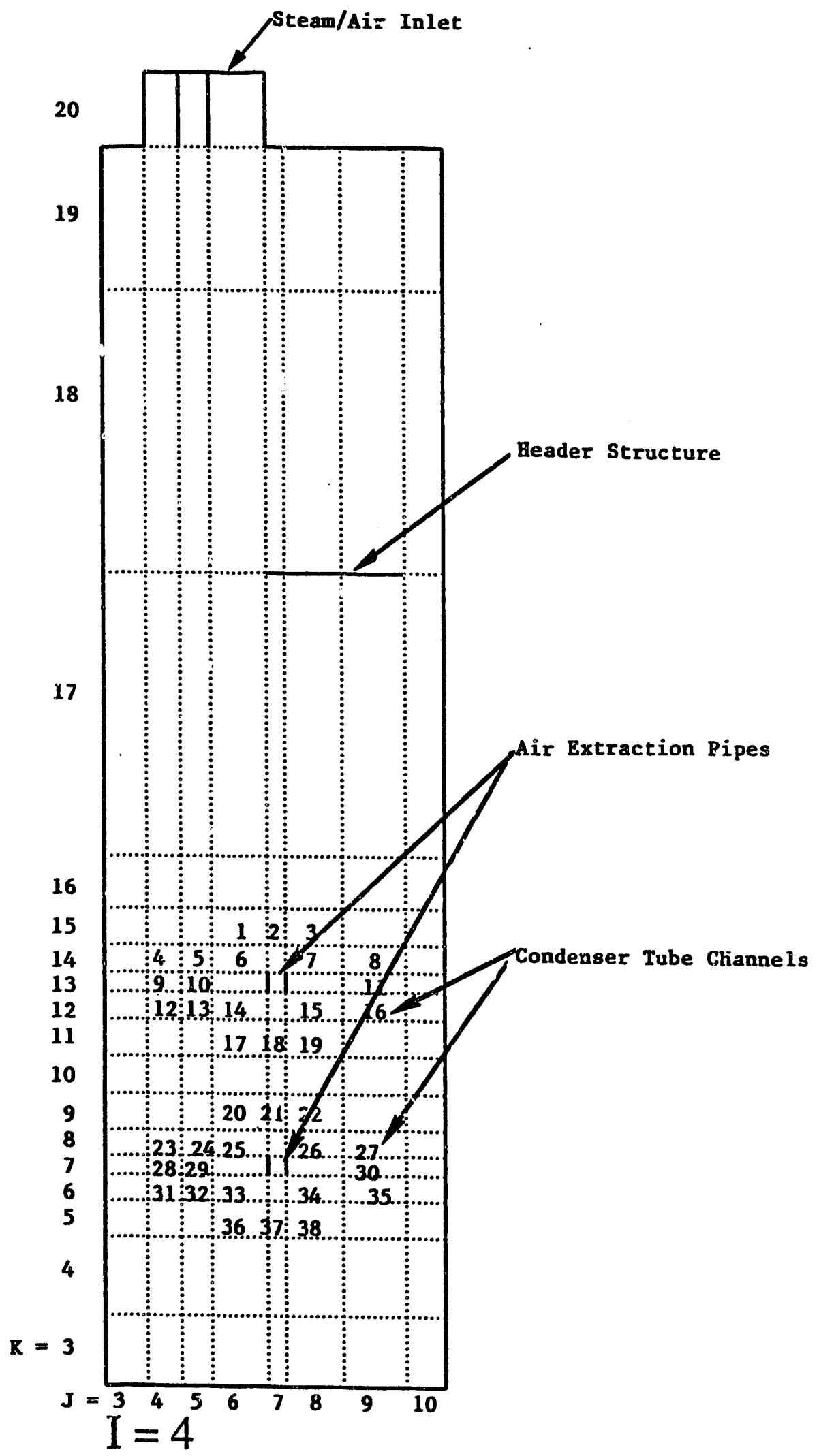

Fig. D.2. Grid layout at the $I=4$ plane 


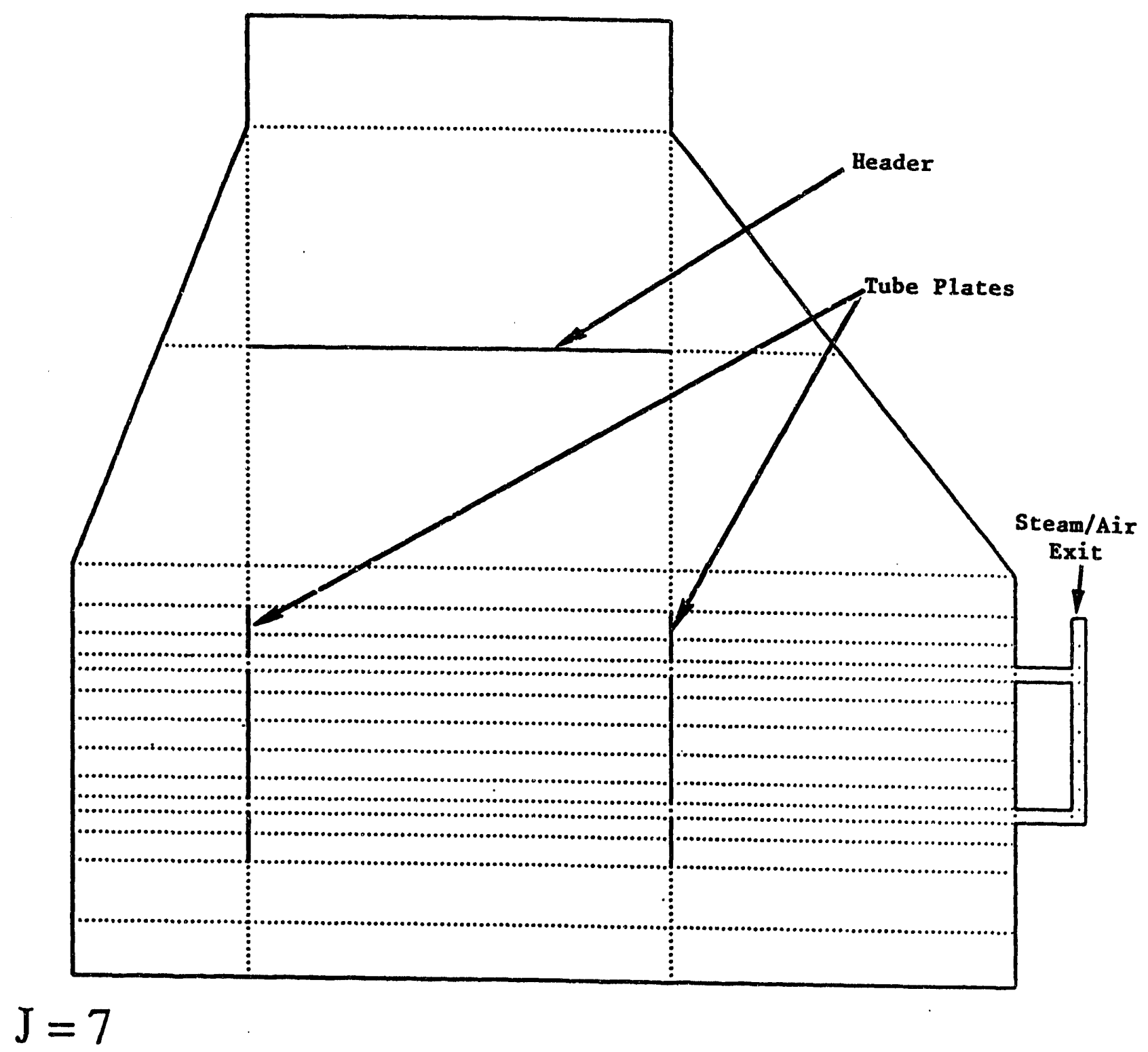

Fig. D.3. Grid layout at the $J=7$ plane 


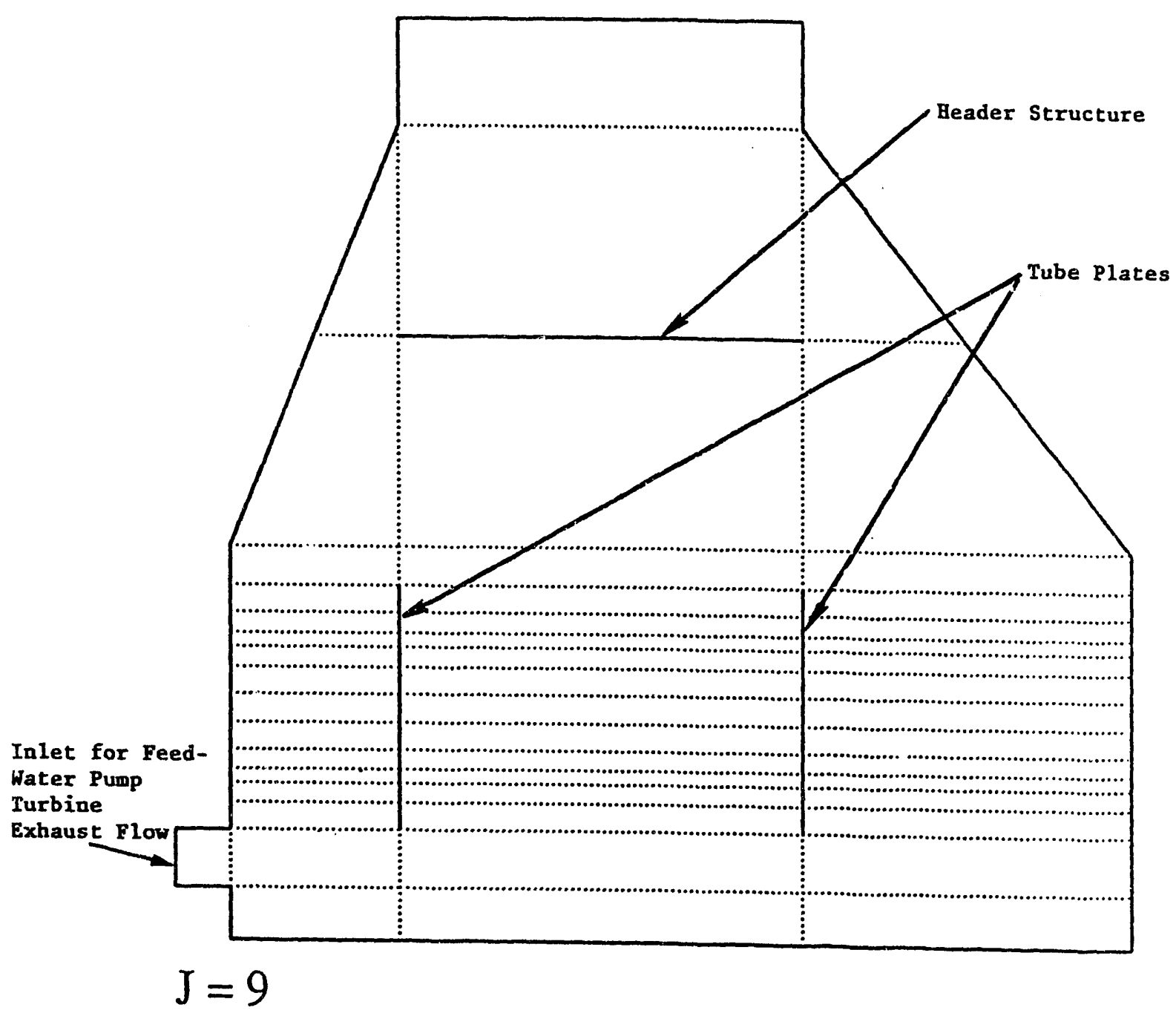

Fig. D.4. Grid layout at the $J=9$ plane

\section{D.3.3 Flow Boundary Conditions}

Shell side

Inlet: Uniform steam and air mixture flow with air mass fraction equal to $4.105 \times 10^{-5}$ enters through the top; constant steam mass flow equal to $5.6 \mathrm{~kg} / \mathrm{s}$ enters through the lower portion of the condenser.

Outlet: Constant mass flow of steam/air mixture, $0.0505 \mathrm{~kg} / \mathrm{s}$.

Walls: No slip.

Symmetric plane: Free slip. 
Tube side

Inlet: Constant velocity, $2.0028 \mathrm{~m} / \mathrm{s}$.

Outlet: Continuity mass flow.

\section{D.3.4 Thermal Boundary Conditions}

Shell side

Inlet: Constant temperature, $46.983^{\circ} \mathrm{C}$ at the top and $46.22^{\circ} \mathrm{C}$ at lower portion of the condenser.

Outlet and Other Walls: Adiabatic.

Tube side

Inlet: Constant temperature, $29^{\circ} \mathrm{C}$.

Outlet: Adiabatic.

\section{D.3.5 Pressure Boundary Condition}

Shell side

Inlet: Constant pressure at top, 10.577 Pa.

Tube side

Constant pressure at inlet, 94,006 $\mathrm{Pa}$.

\section{D.3.6 Force Resistance Correlations}

Shell side

Tube Bundle: Crossflow over staggered tubes by Jakob,C.1 Eq. C.2.

Steam/Air to Air Extraction Pipe: Sharp edge orifice, Eq. C.13.

Flow in Air Extraction Pipe: Flow inside straight duct, Eqs. C. 16 and C.17. Tube side

Flow inside straight duct, Eqs. C.16 and C.17.

\section{D.3.7 Other Options}

Shell Side

Constant turbulence diffusivity model.

DMIM (Direct matrix inversion method) solver. 

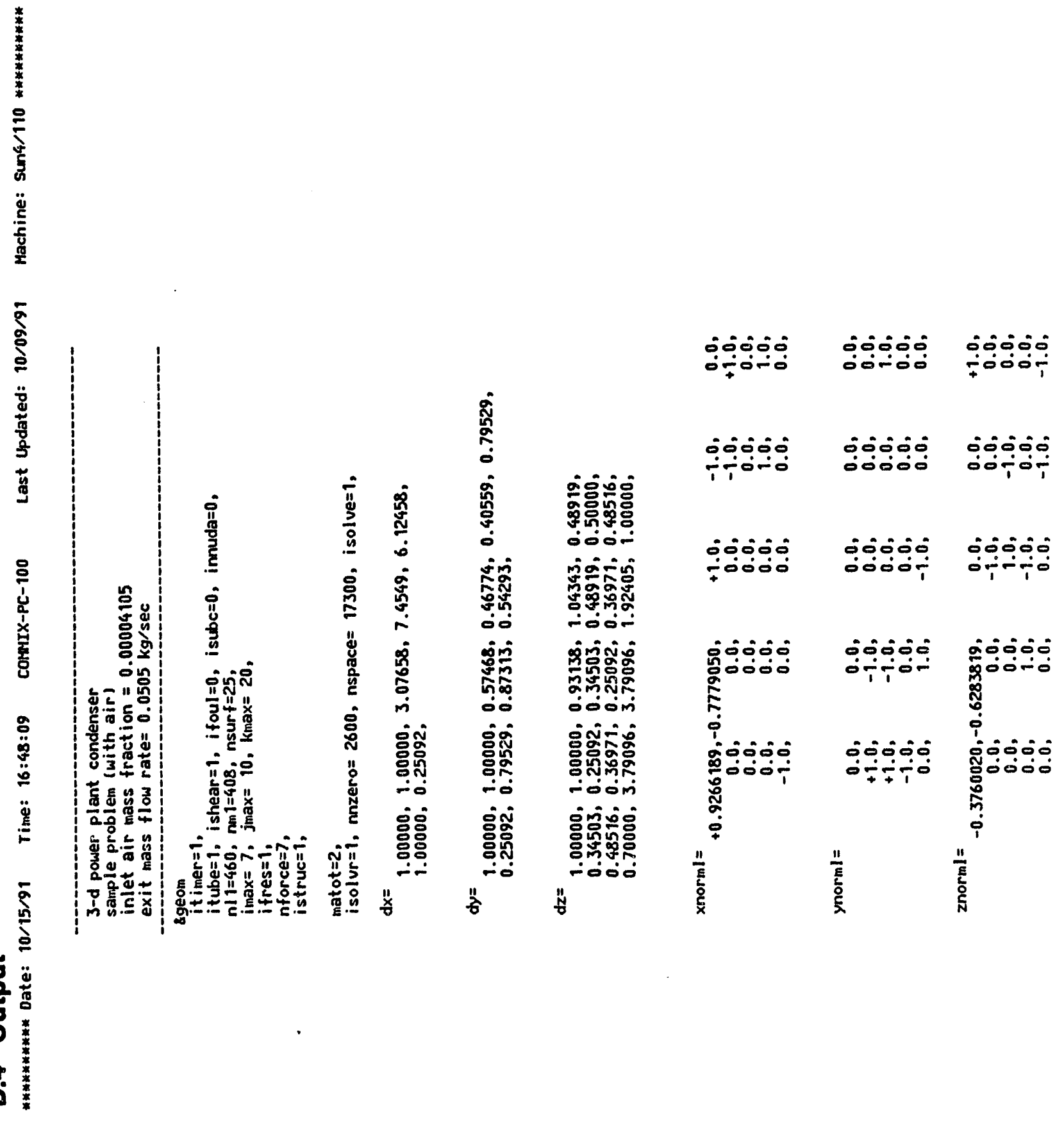
$\overline{\frac{\pi}{3}} \overline{\frac{\pi}{3}}$

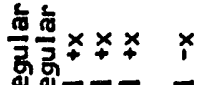

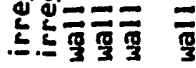

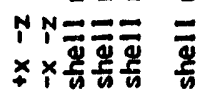

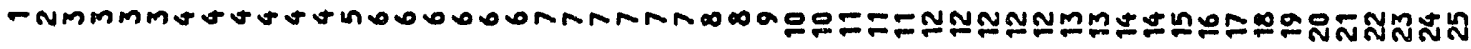

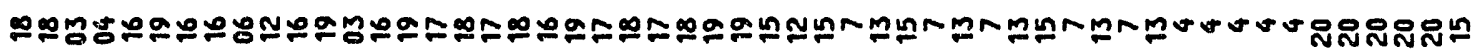

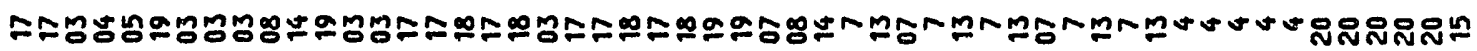

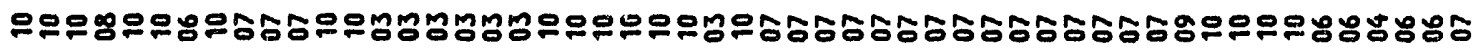

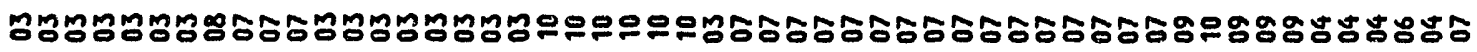

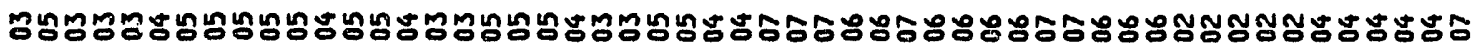

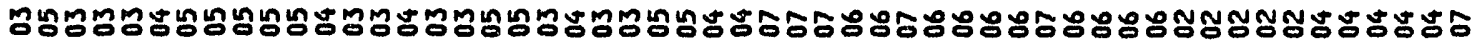

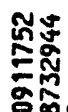

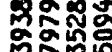




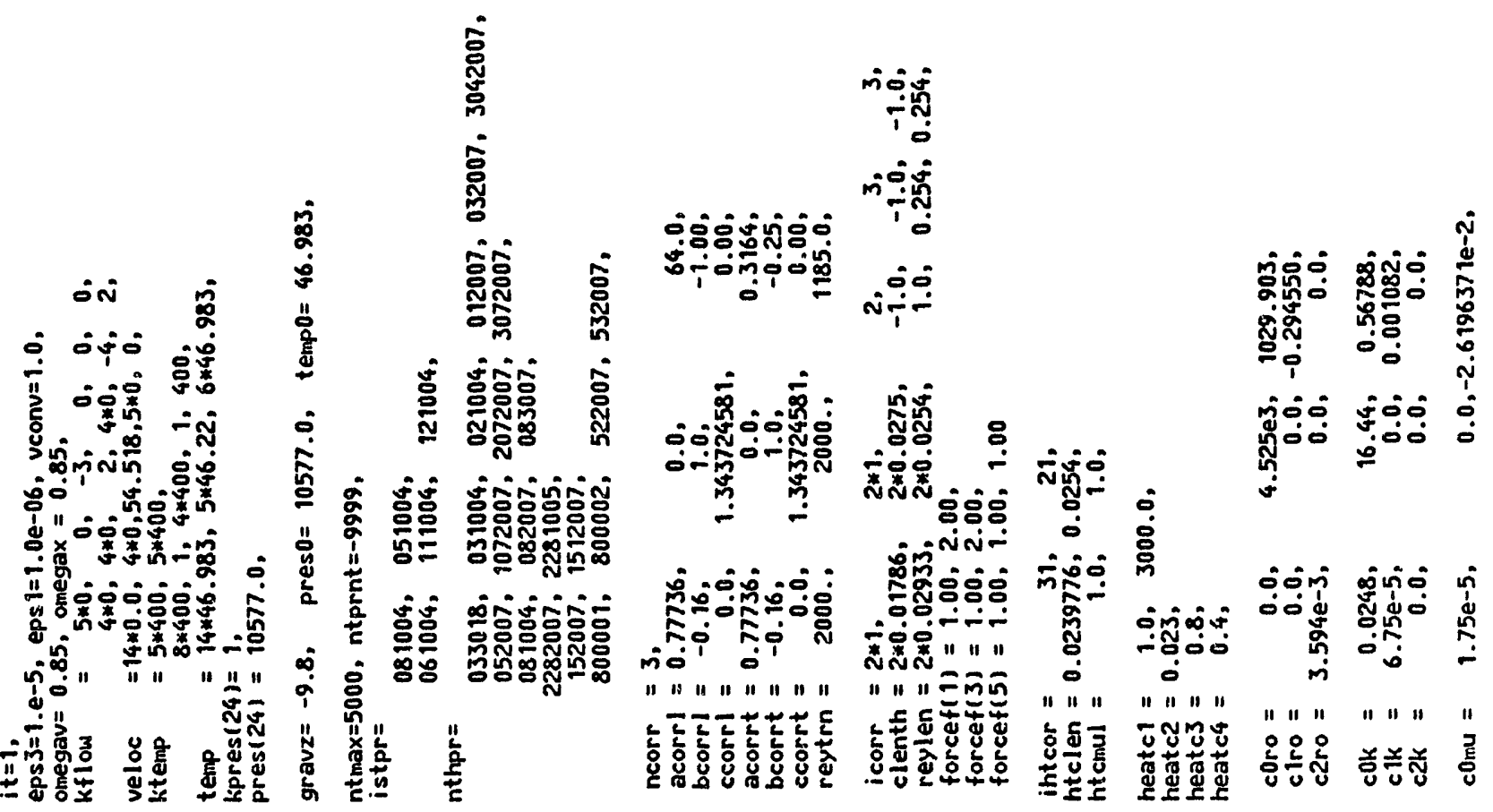




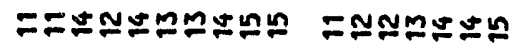

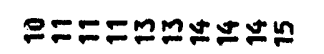

テニッேะே

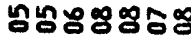

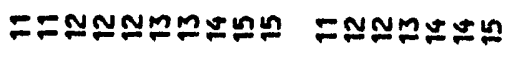

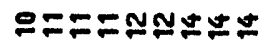

=ニNㅝㄴ

แก่ำะะละ

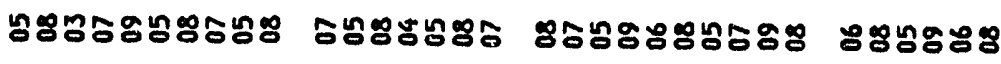

농ํㅗㅇํํㅇ영ํํㅇ

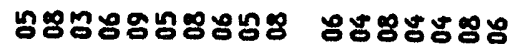

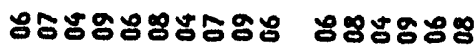

농뿡ํํㅇ영응

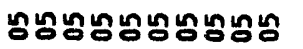

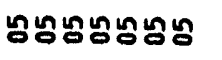

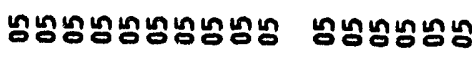

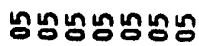
ม่งก่

" "

iा งก้

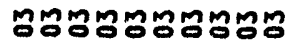

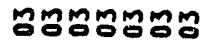

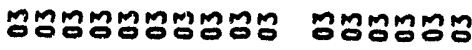

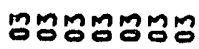

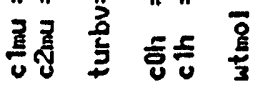

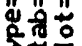




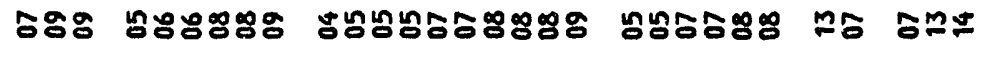

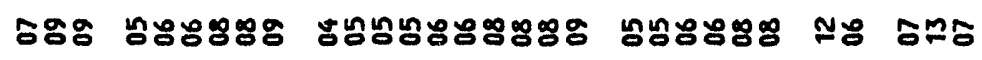

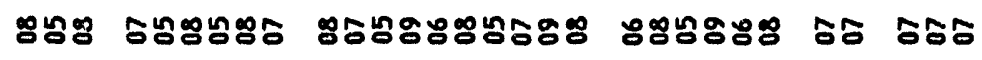

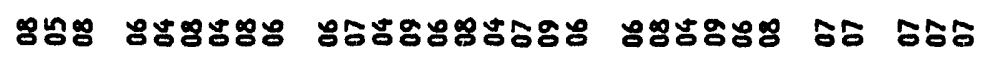

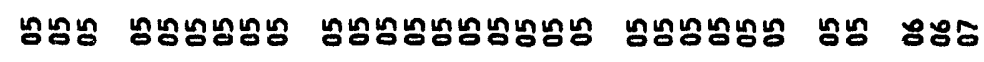

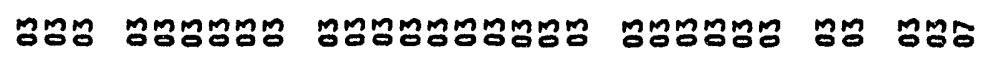

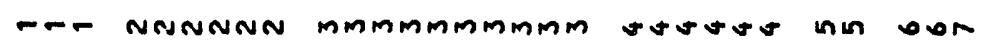

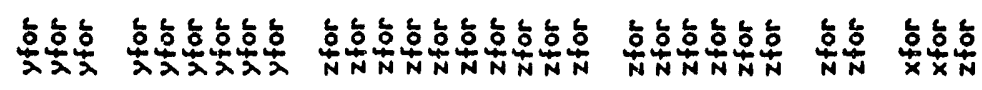

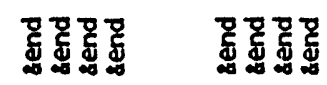

草

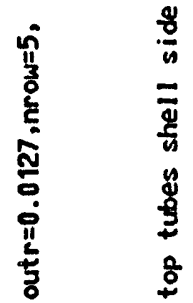

宓

官

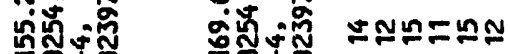

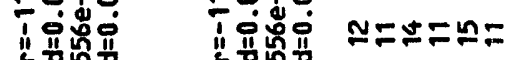

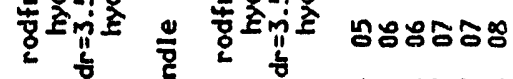

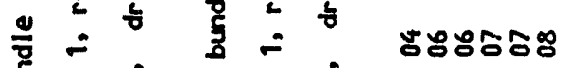
雚 -กิn-

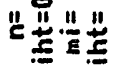

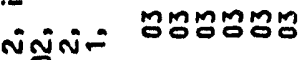
"I"." 至

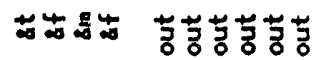




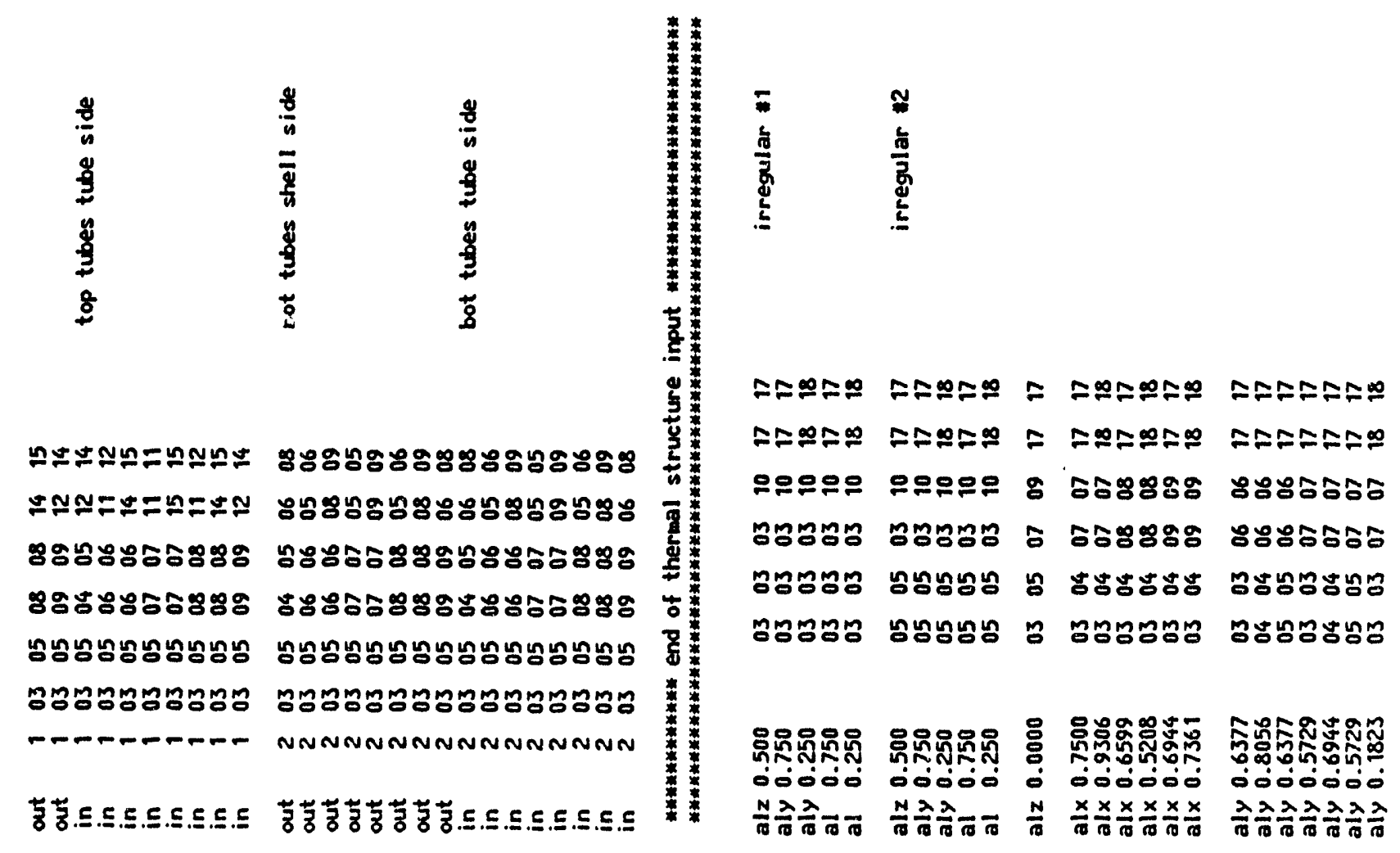




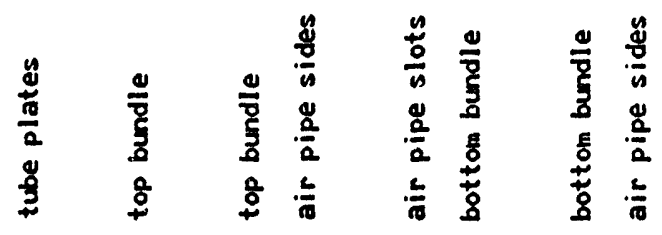

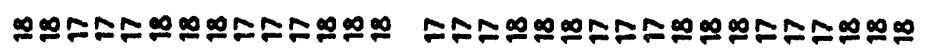

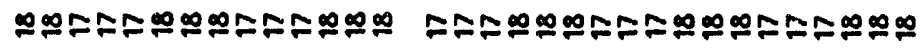

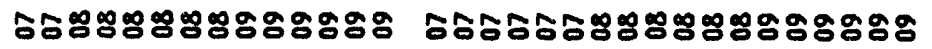

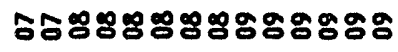

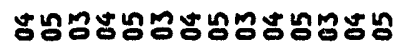

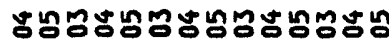

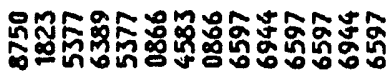

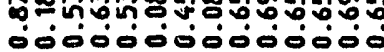

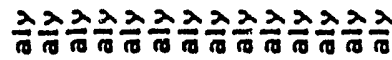

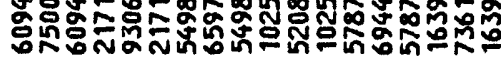

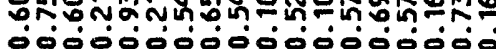

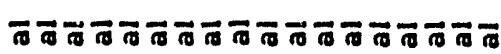

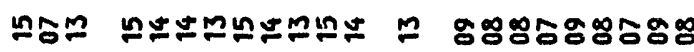

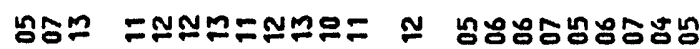

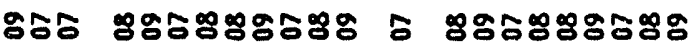

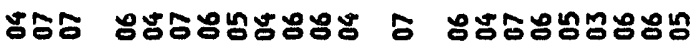

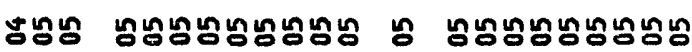

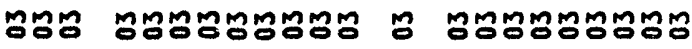
$\ddot{0} \rightarrow=$ $\frac{x}{10} \frac{x}{\pi}$
สิ้สูก 敛 ถูก๊

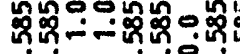

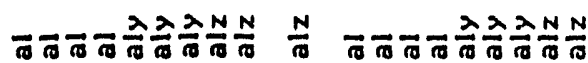




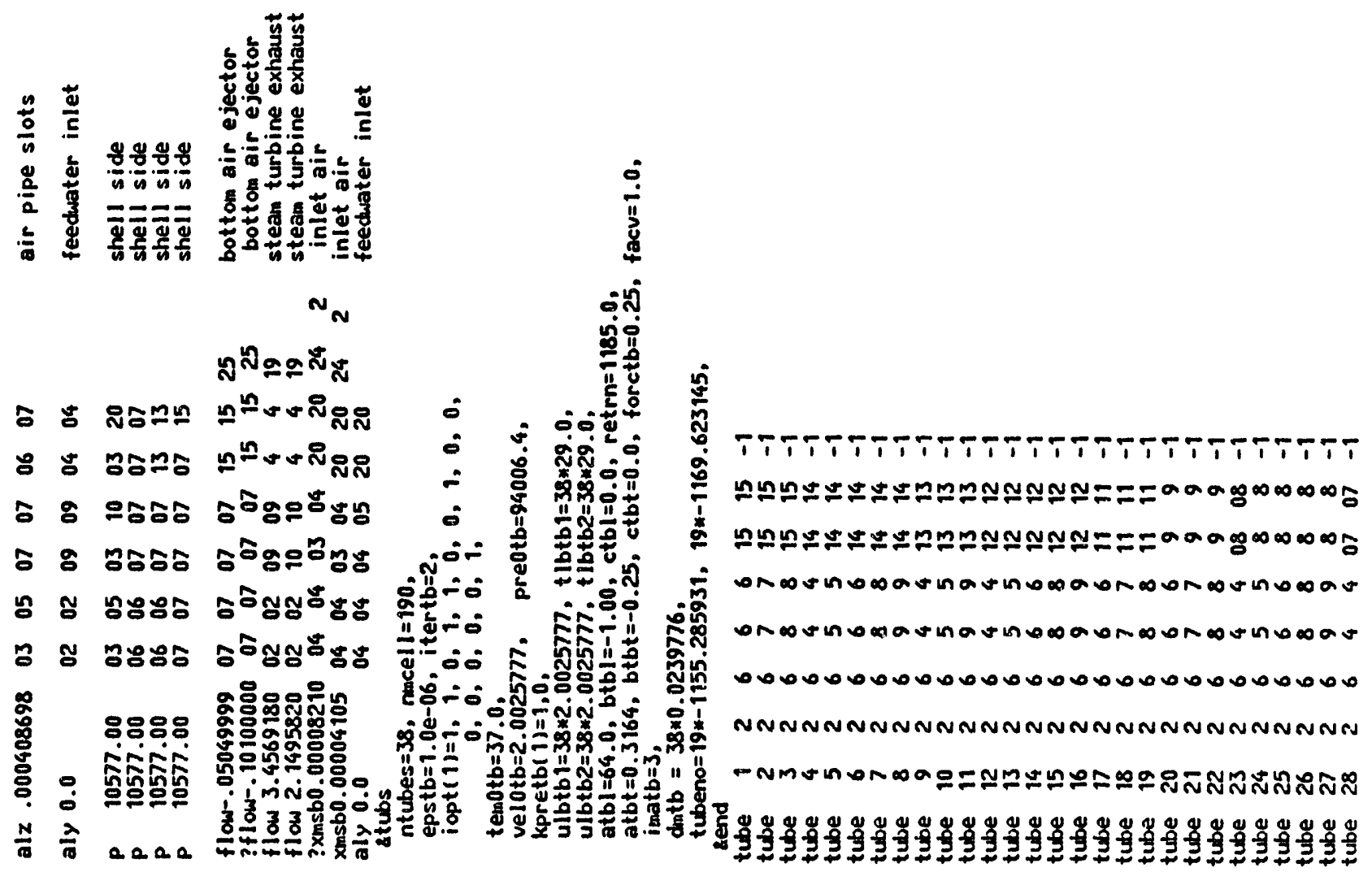




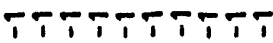

nNg0000num nNg0000unin nasunesano

natinomano $\bullet \bullet \bullet \bullet \bullet \bullet \bullet \bullet \bullet \bullet$ NNNNNNNNNN

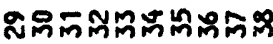

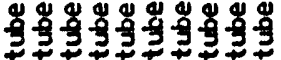



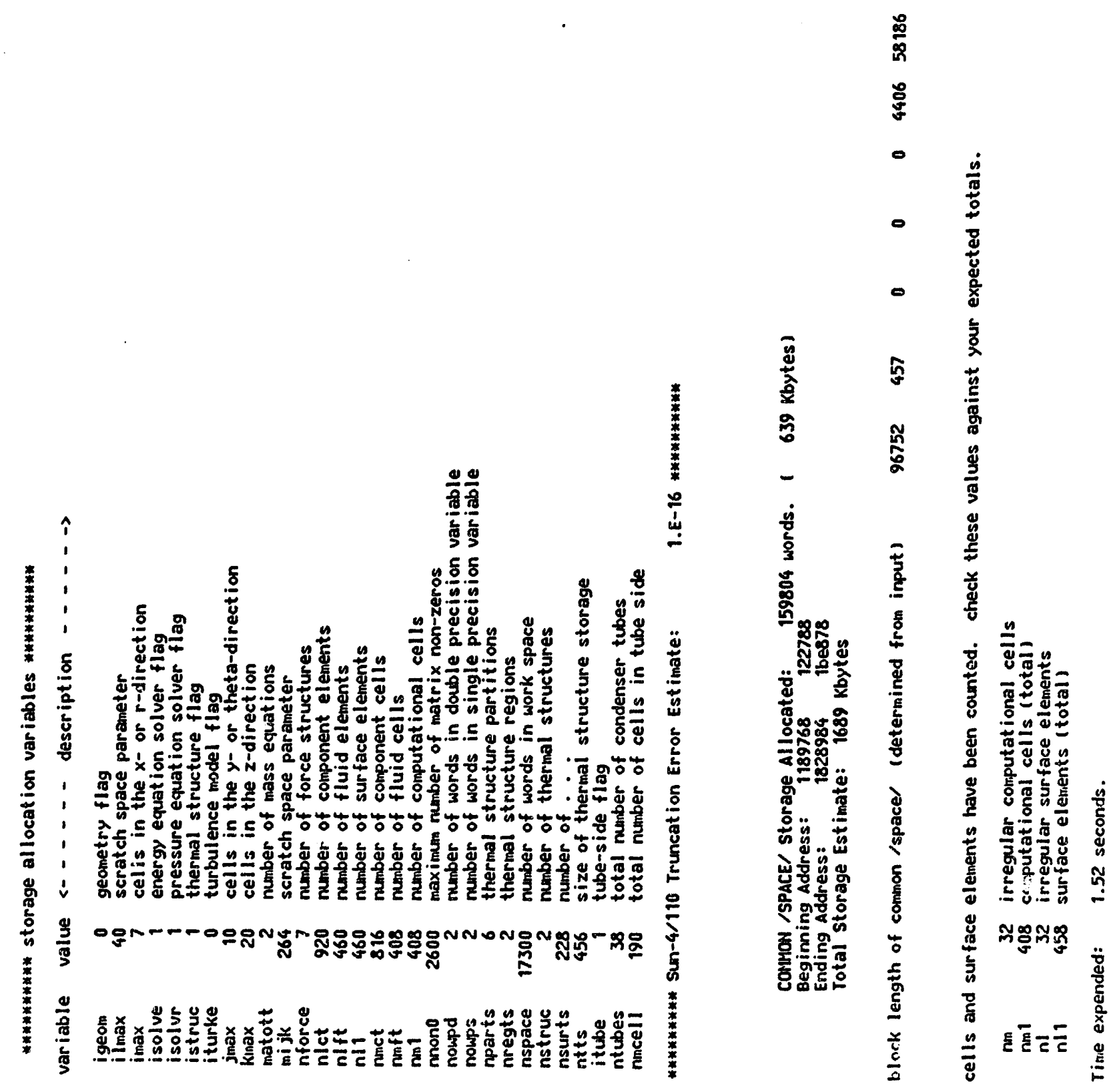


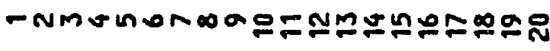
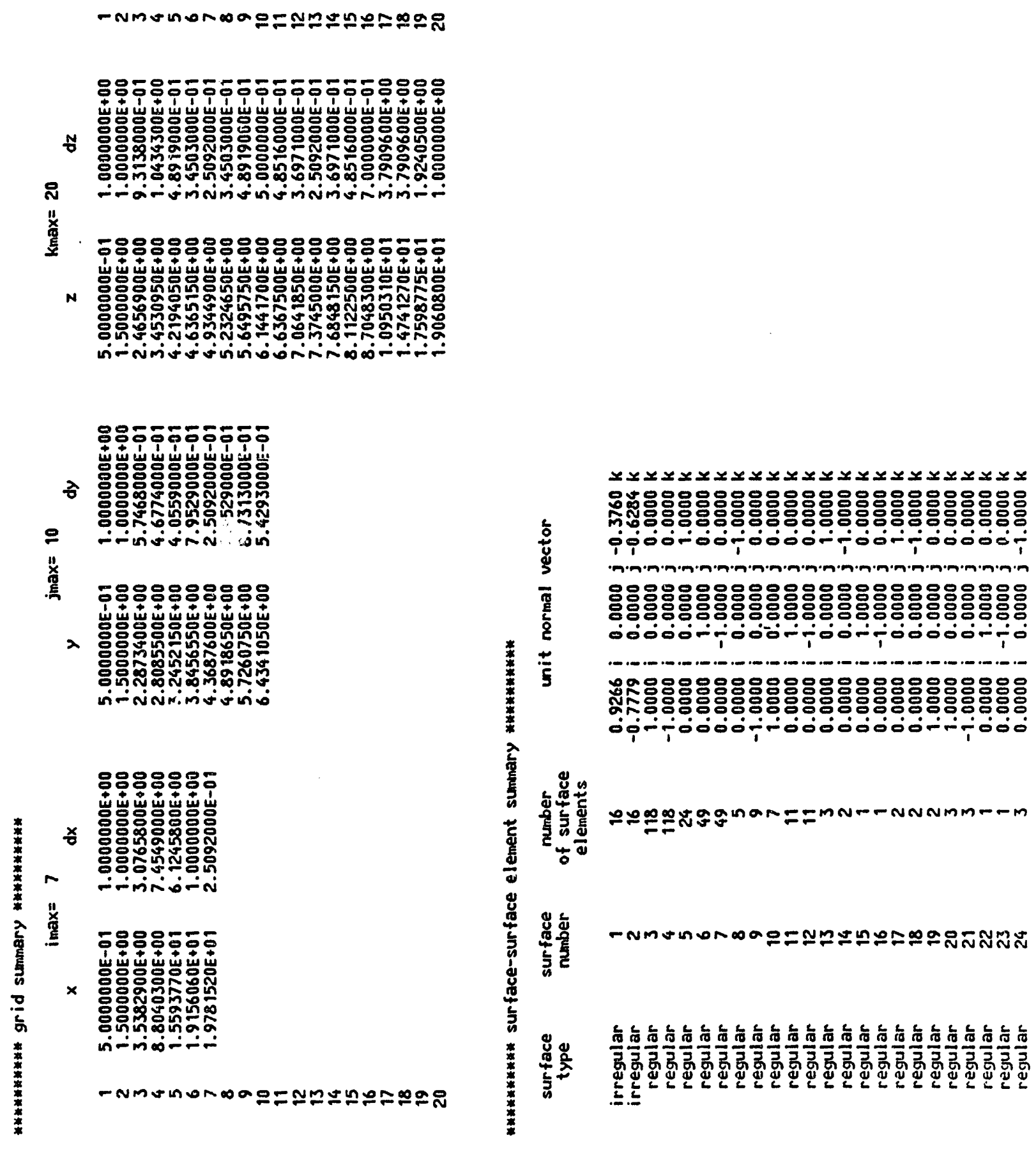

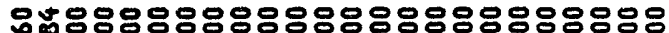

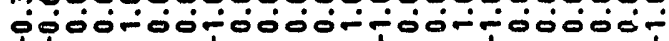
mmmmmmmmmmmmmmmmmmm

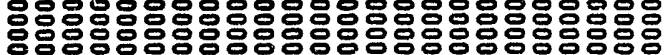

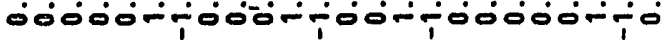

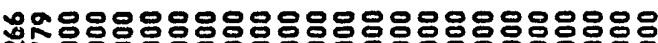

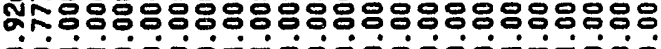
i T0000\%-00000000 T000

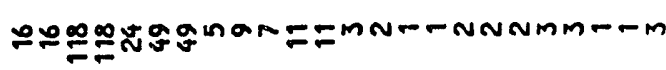

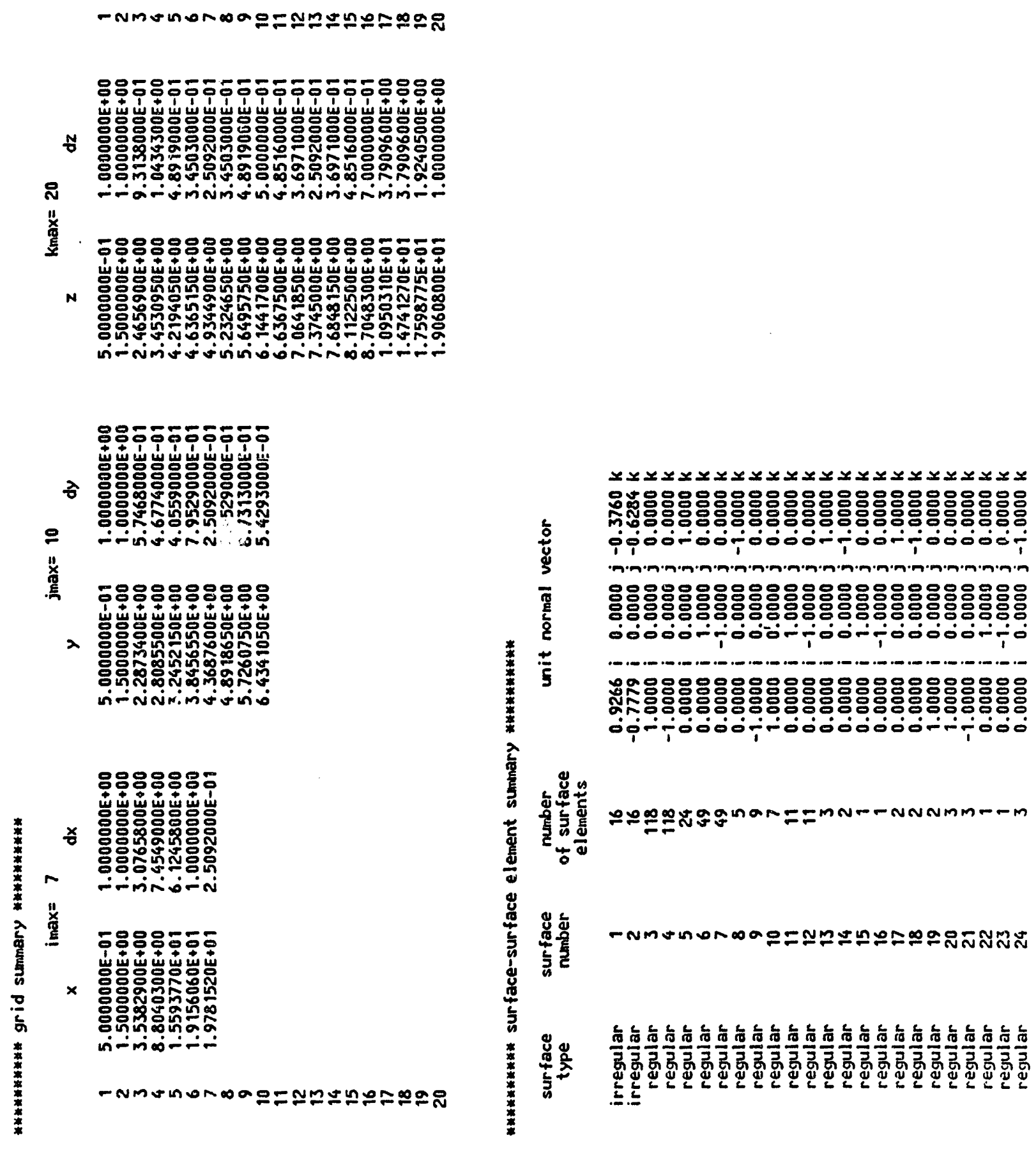

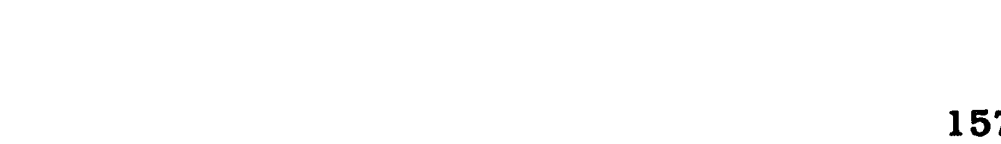


158

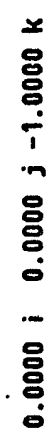

-

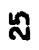

密 
159

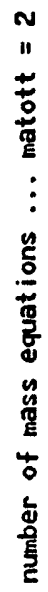




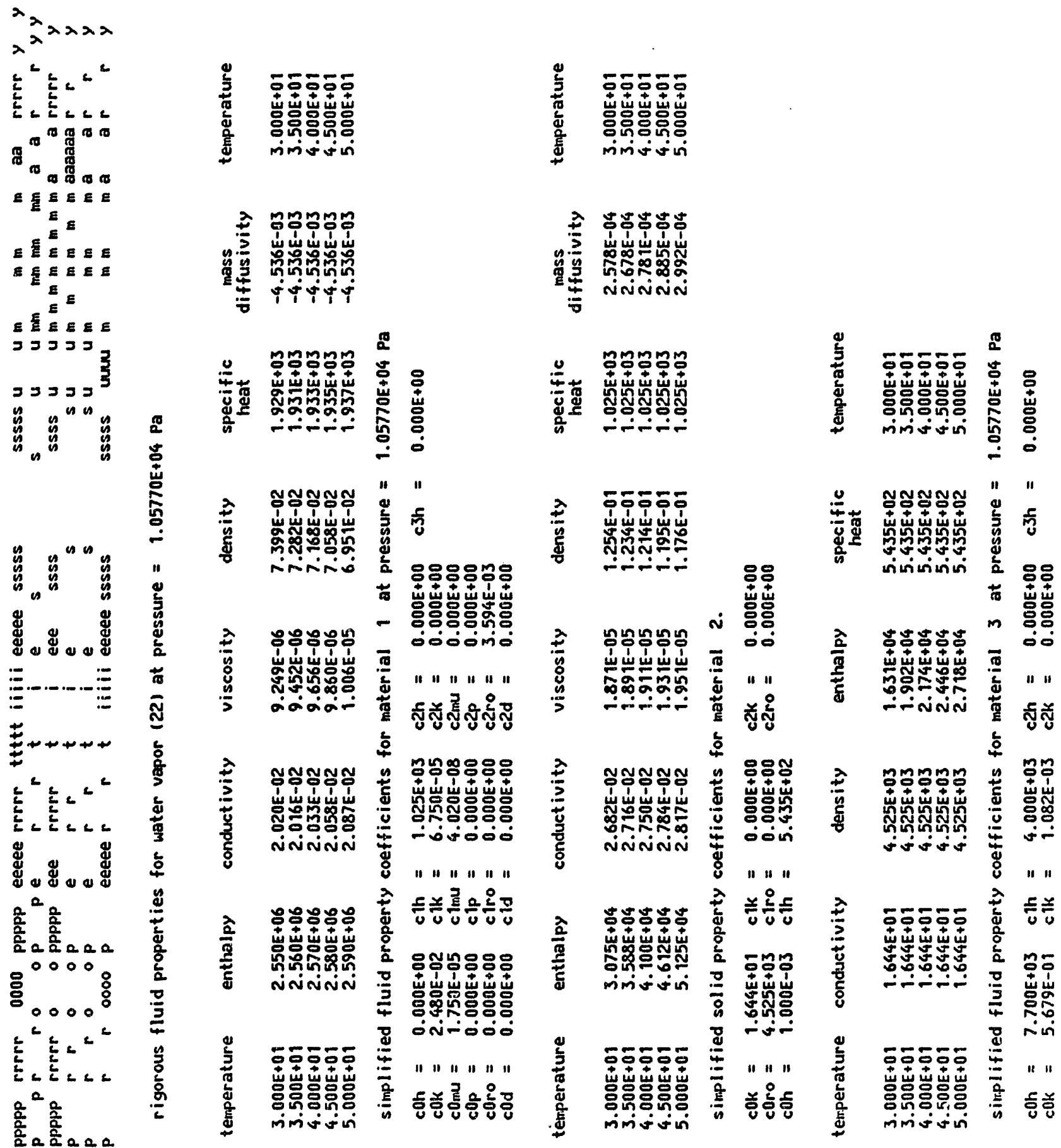



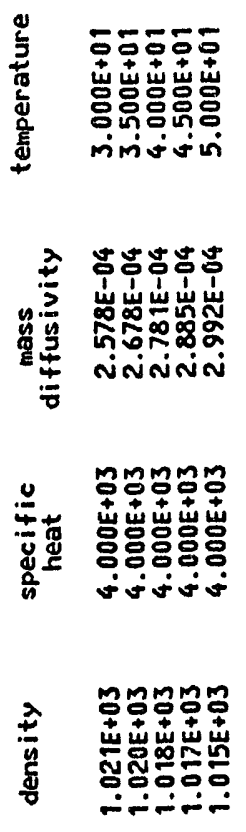

웅용

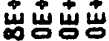

꿍ㅇㅇㅇㅇㅇ

مं்ó

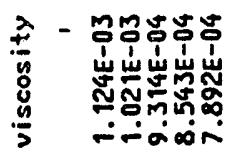

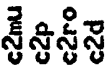

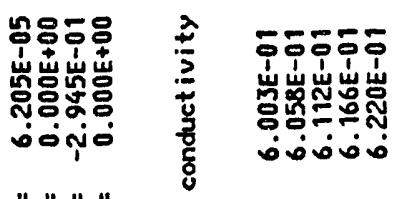

른은 운은

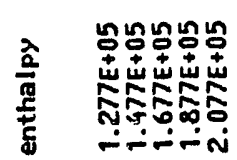

崫出㟧

సูㅇㅇㅇㅇㅇ

พं०-०

" | " "

롱홍원뭉

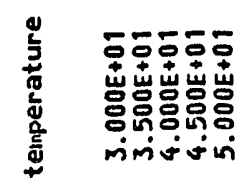




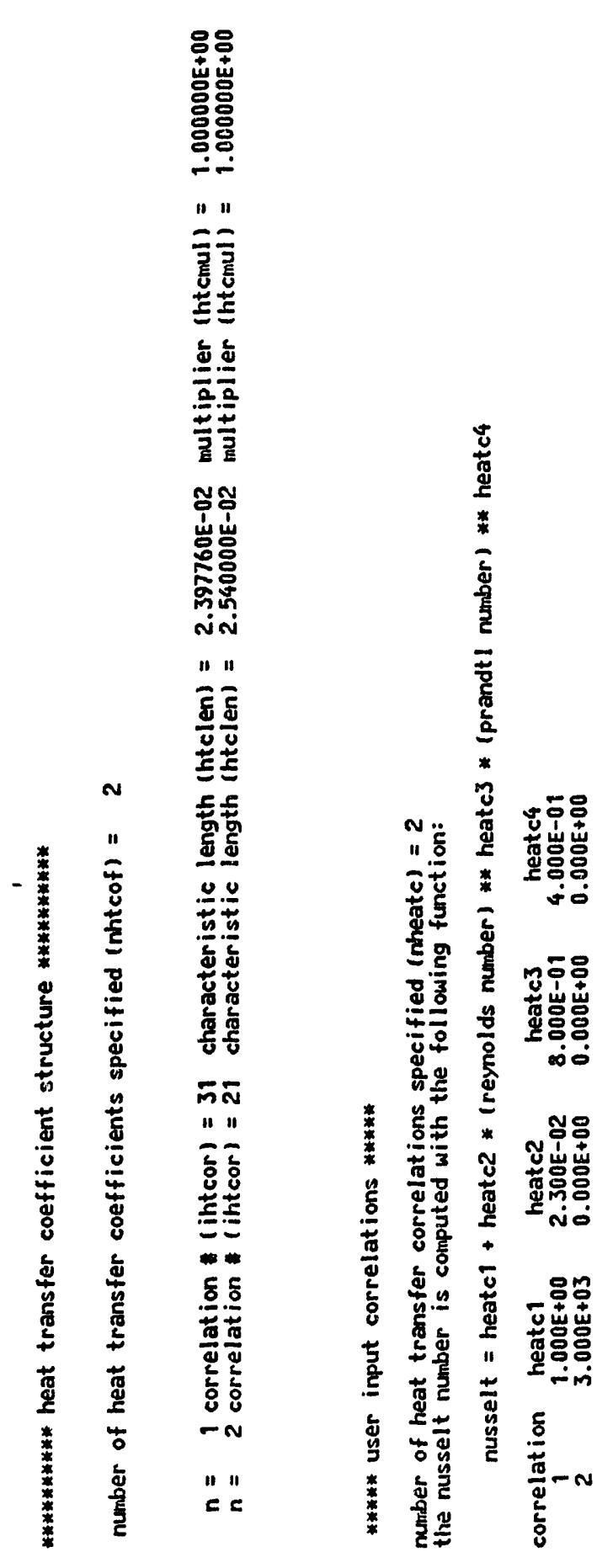




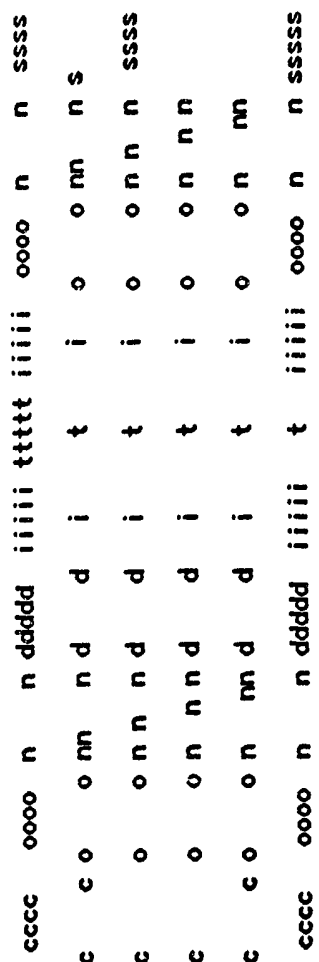

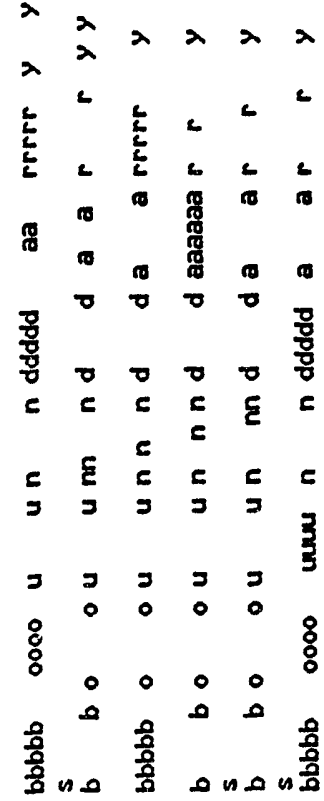
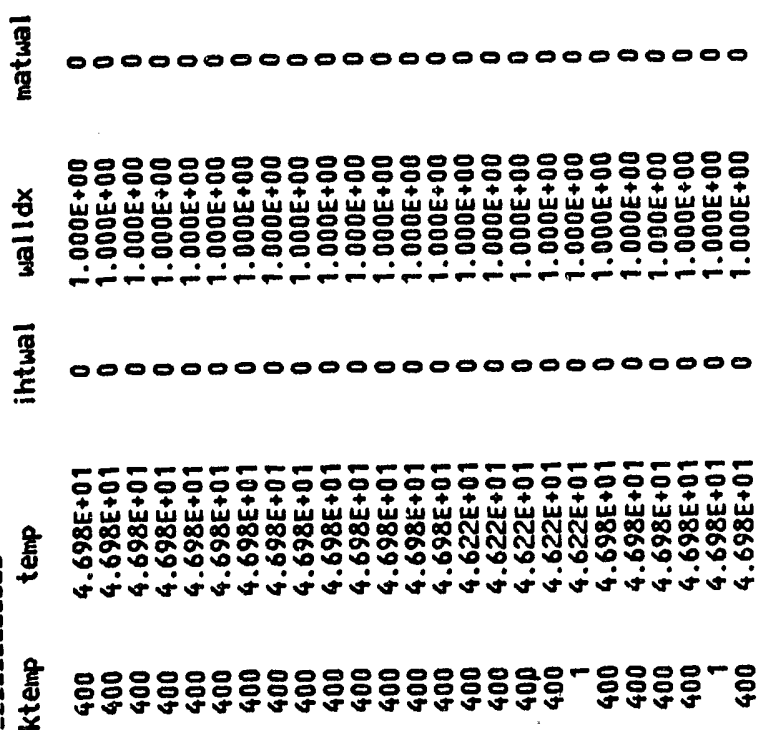

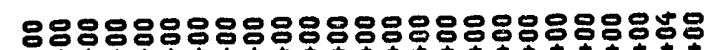

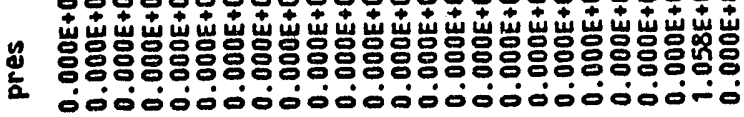

y 0000000000000000000000000

章

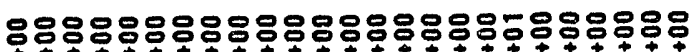

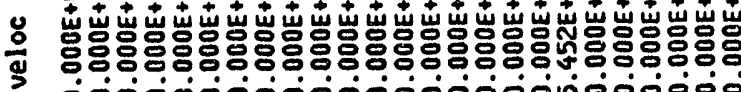

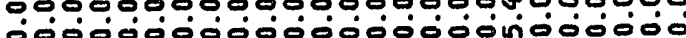

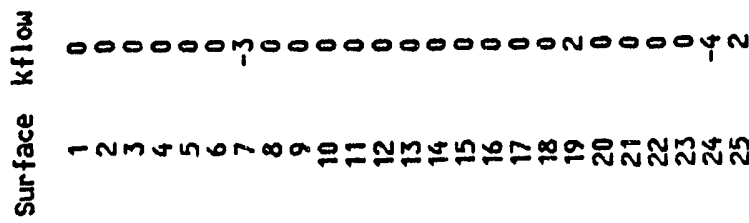

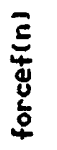

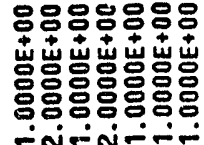

in

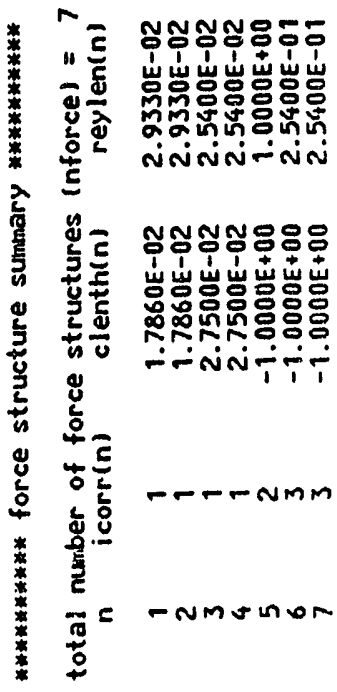



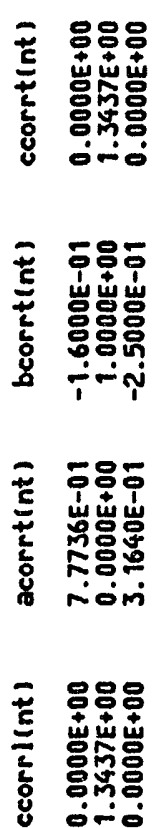

\$

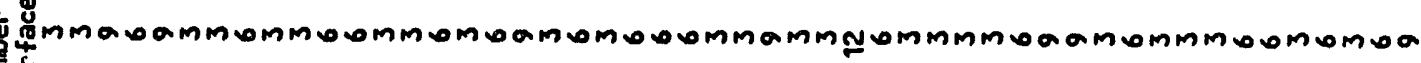
4

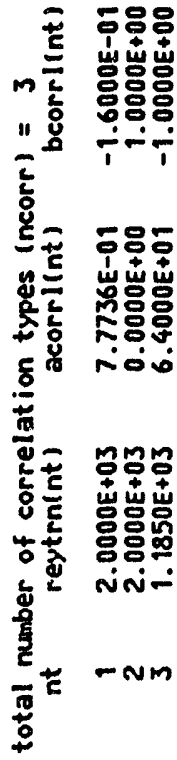

d 密

方至

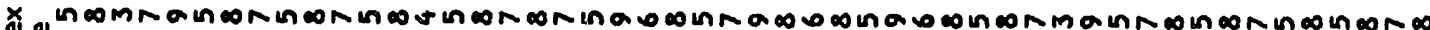
ह

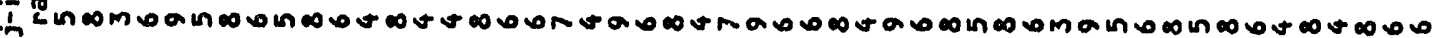

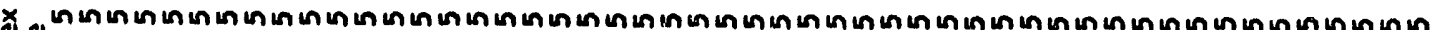
\&

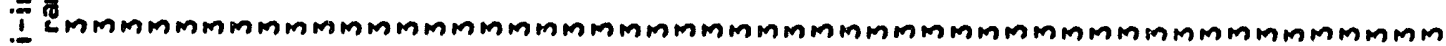

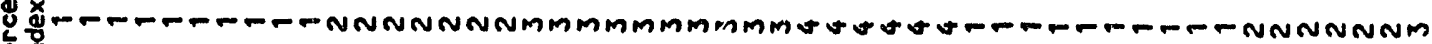
․․․․․

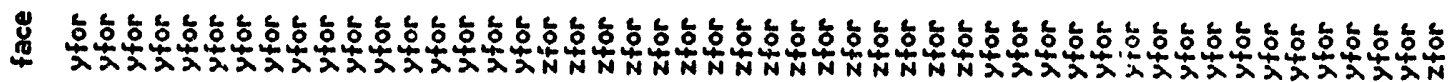


momonommamm Nommonos

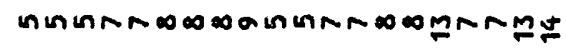

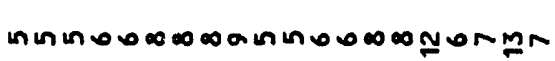

Minaosinnadosinasonnarn

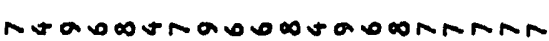

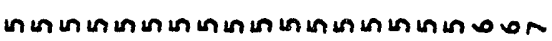

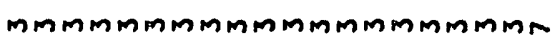

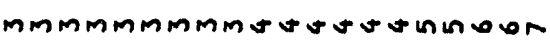

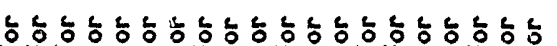
N

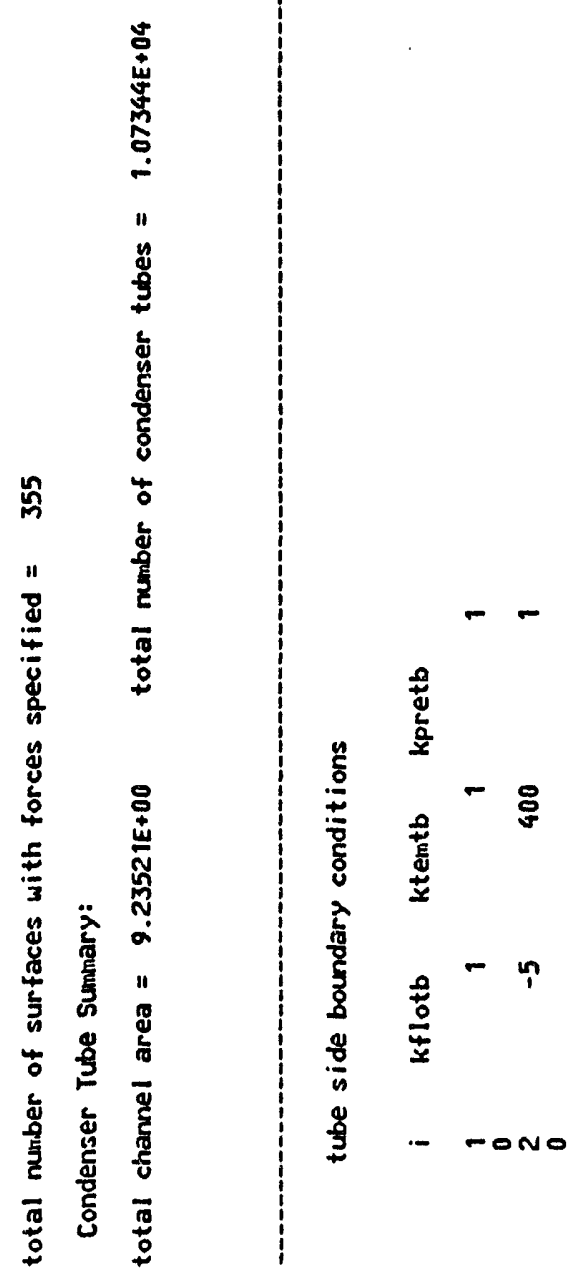



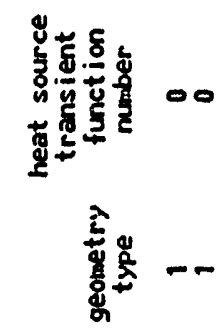

它

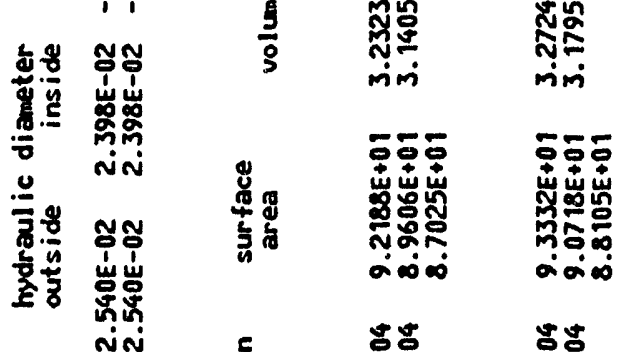

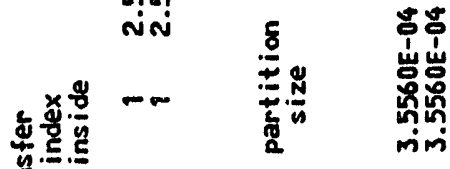

葛离

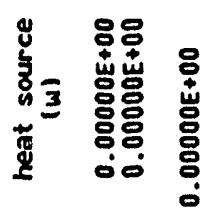

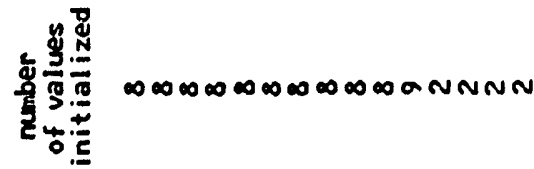

*

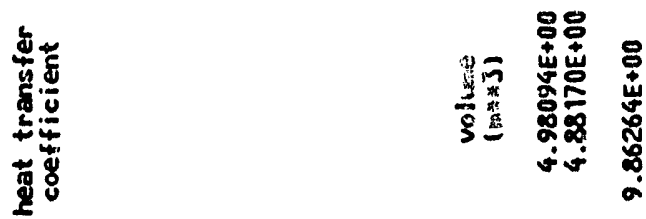

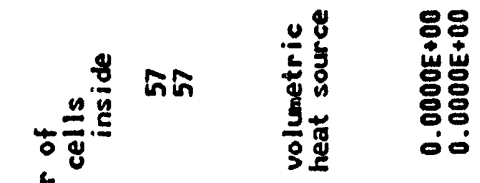

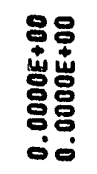

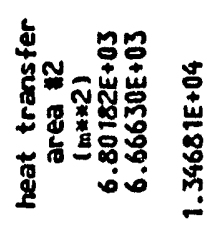

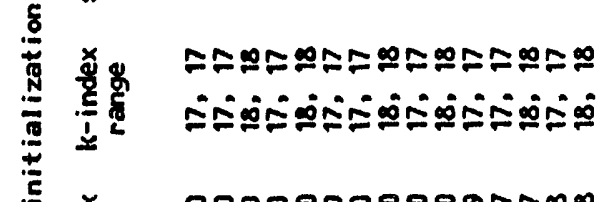

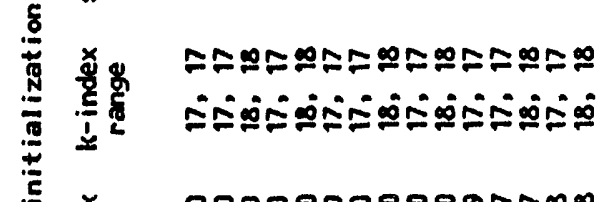

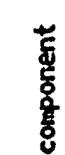

蒿

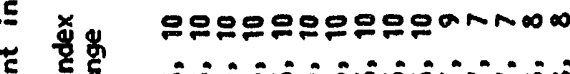

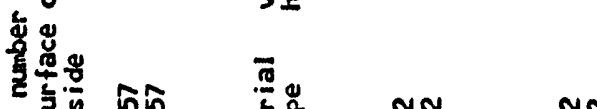

manmminenenengnososo

寒

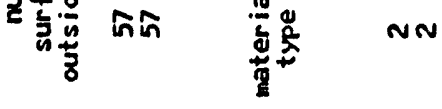

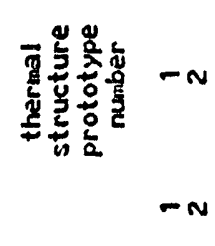

啴-

N

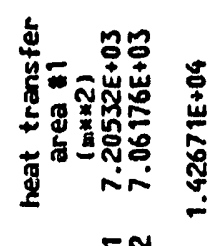

蒙

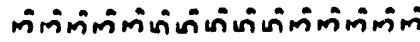

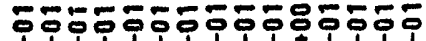

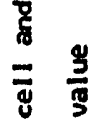

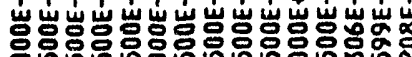

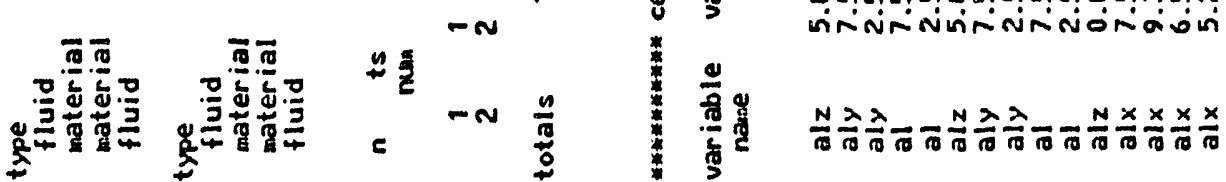




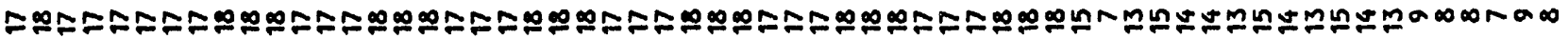

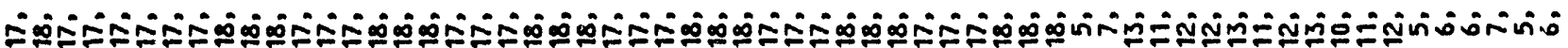

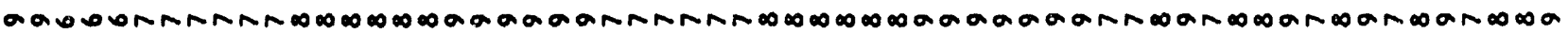

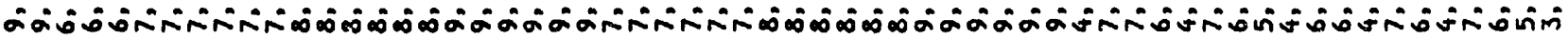

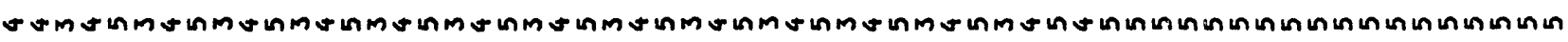

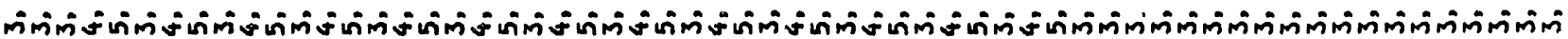

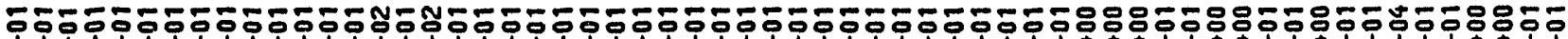

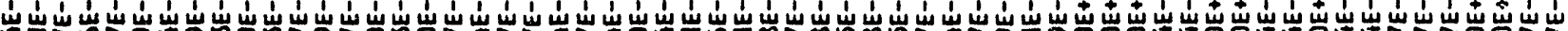

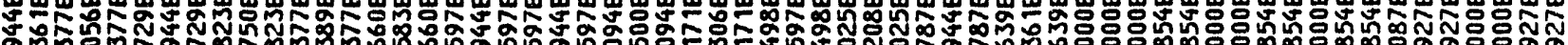
on

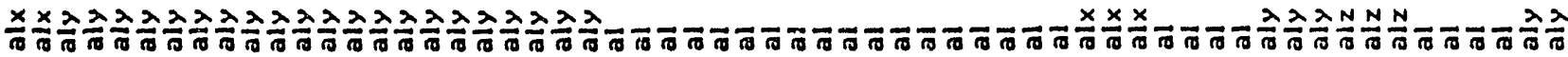




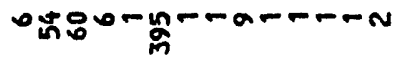

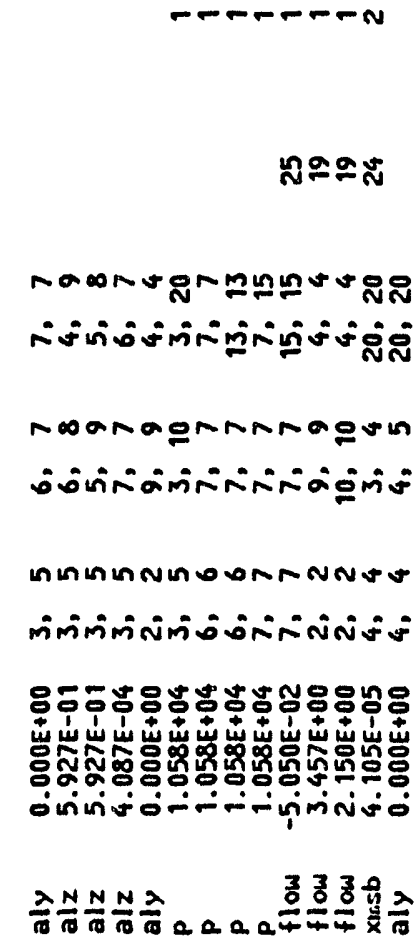

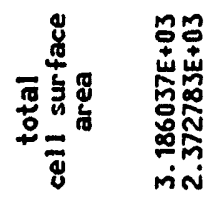
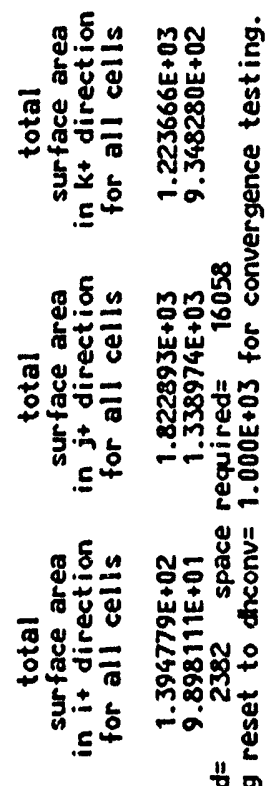

d

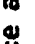

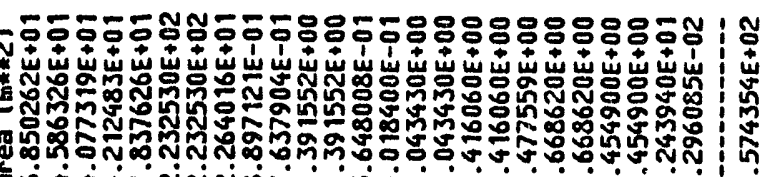

종

오ㅇㅗㅛ

岕岕

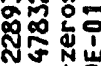

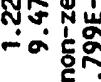

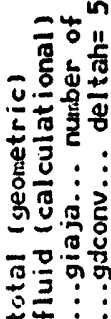



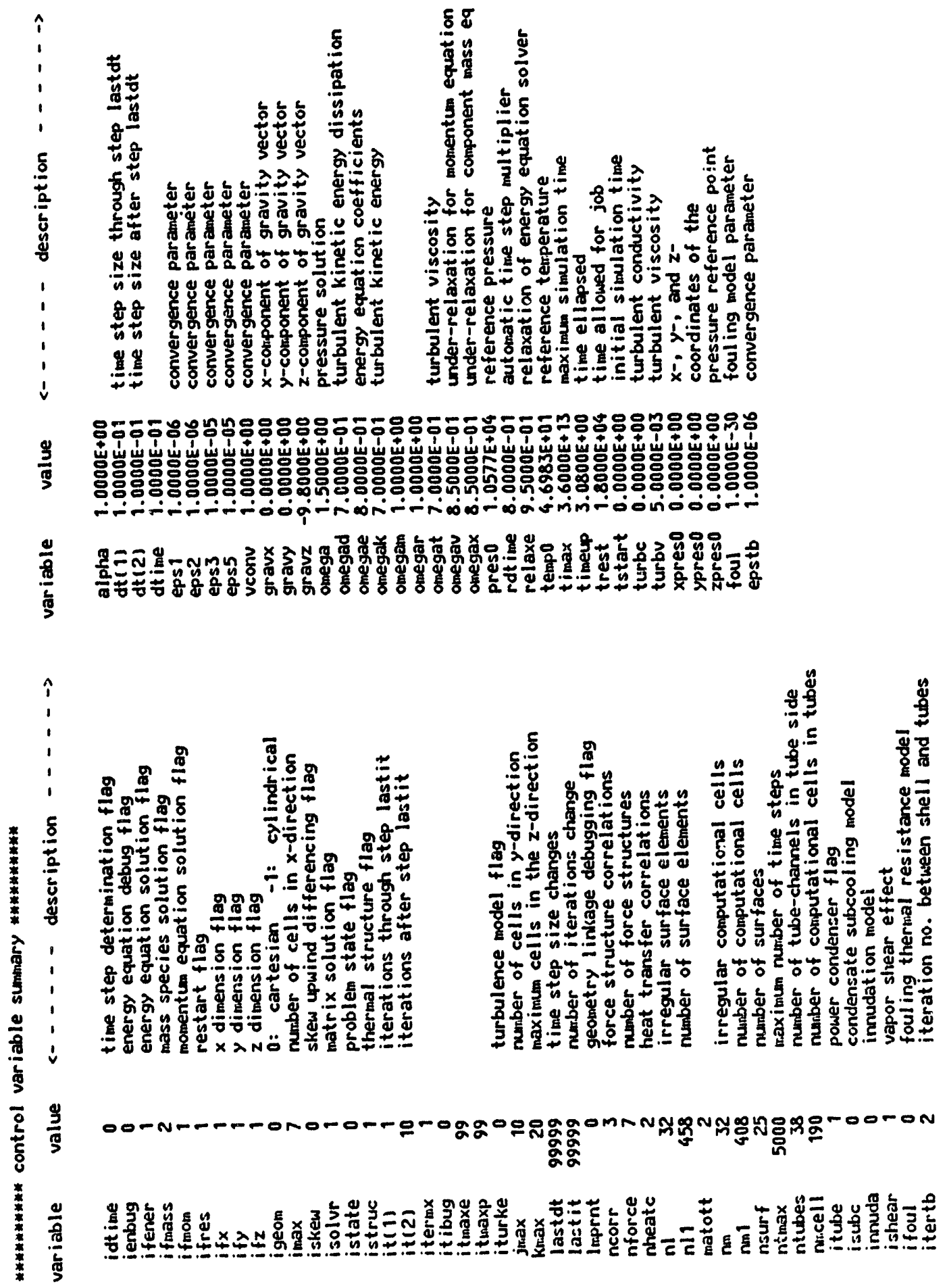

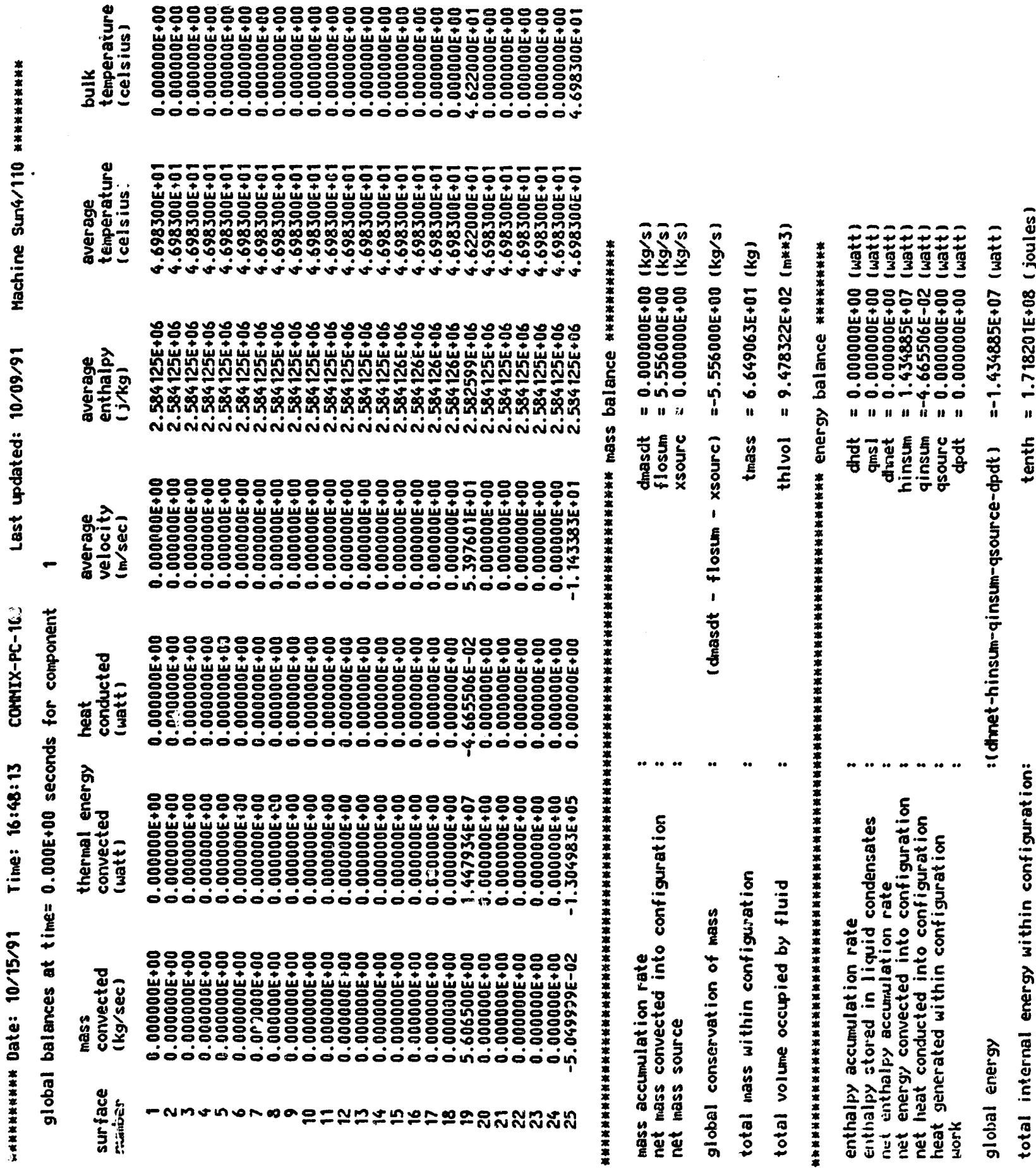


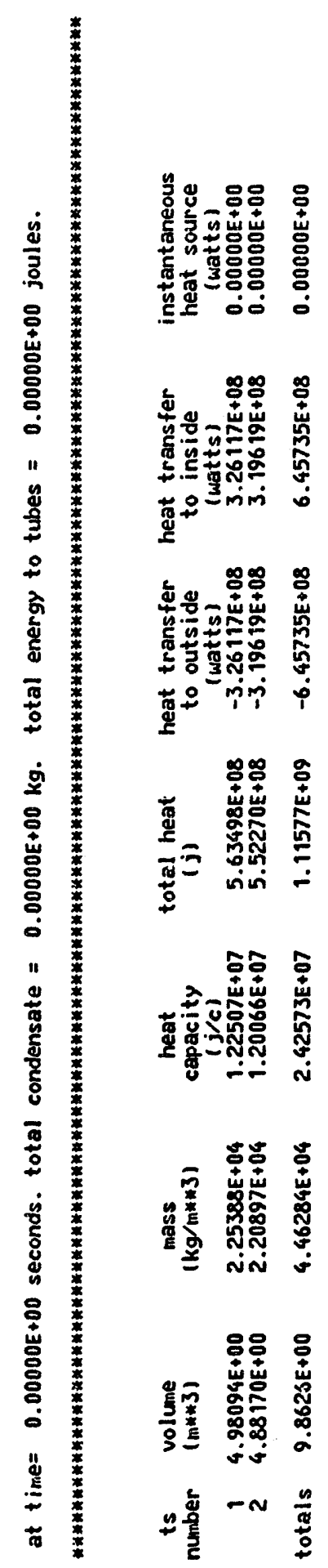



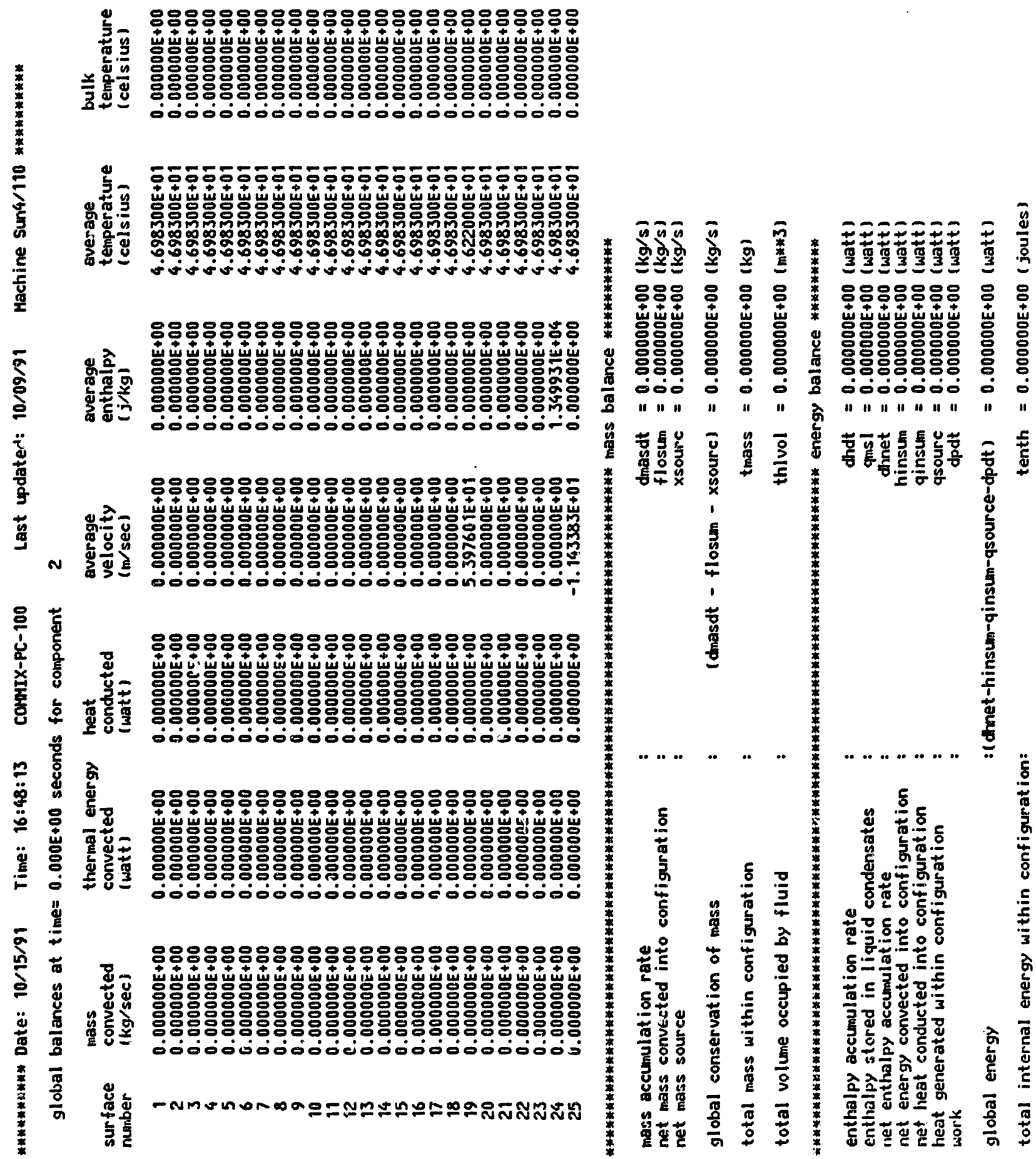


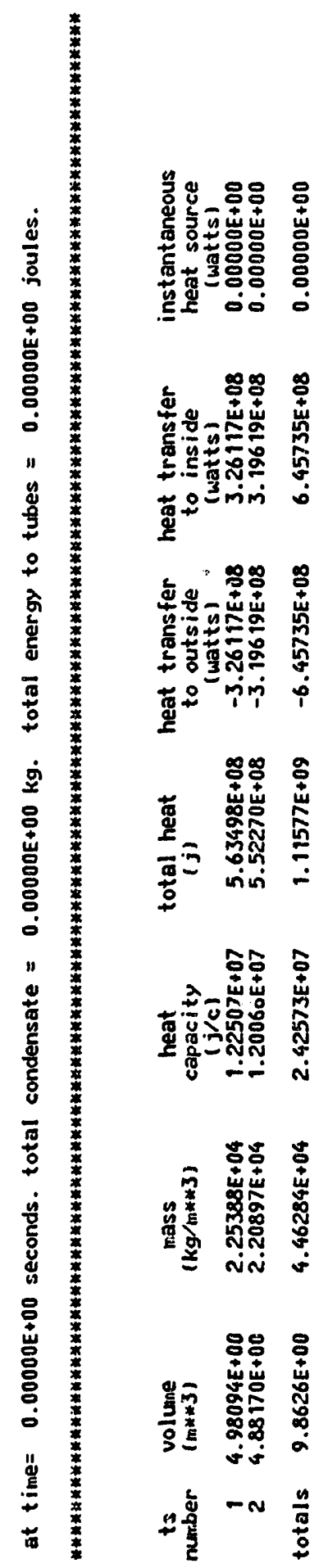



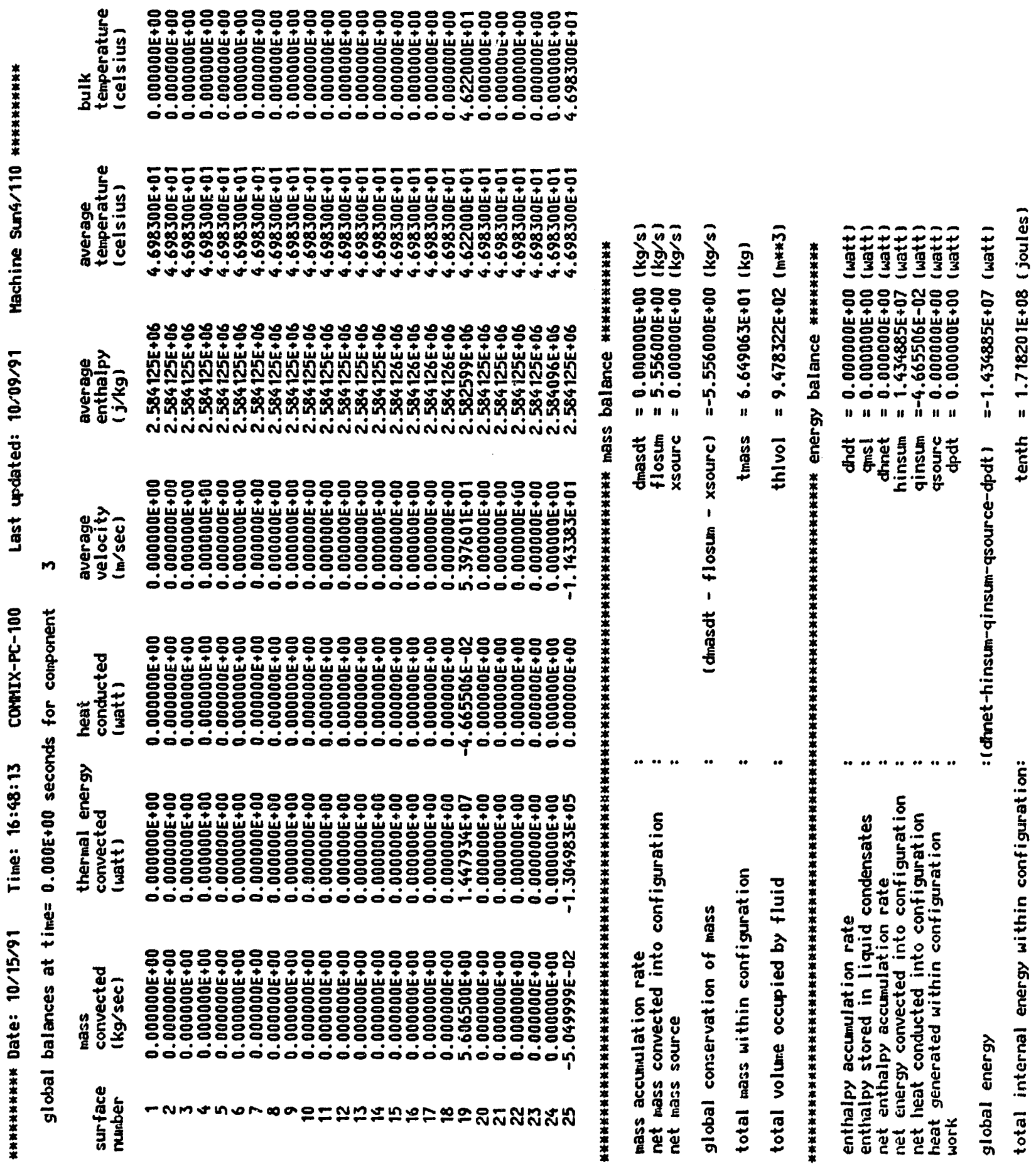
웅

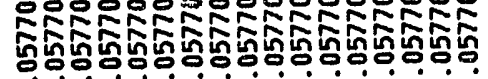

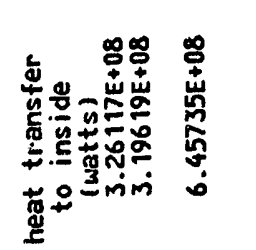

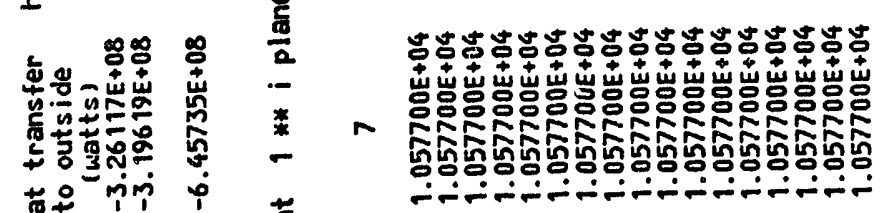

苚诤

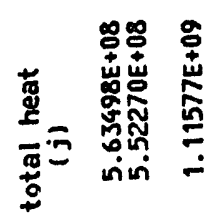

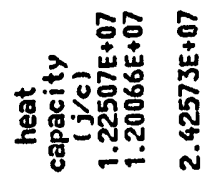

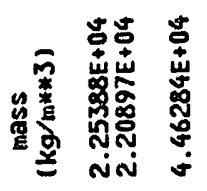

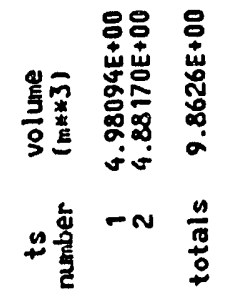

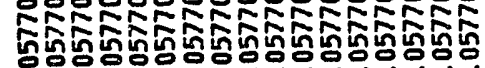

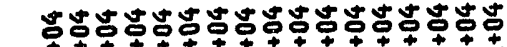

$\infty$

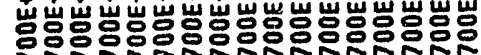

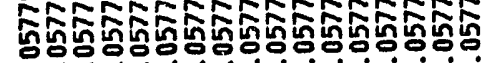

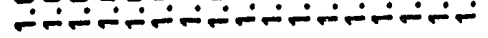

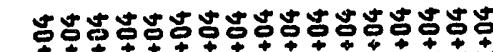

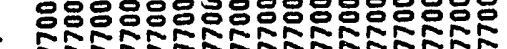

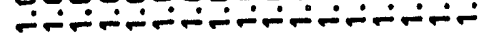

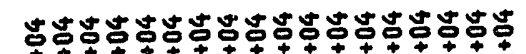

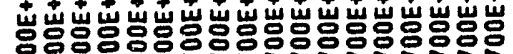

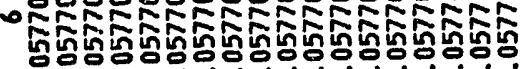
品-

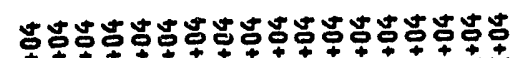

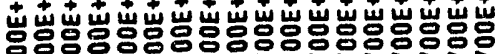

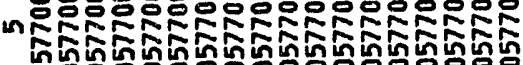

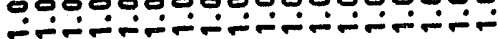

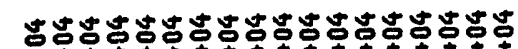

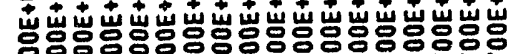

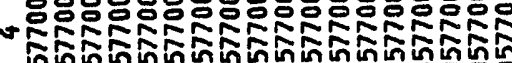

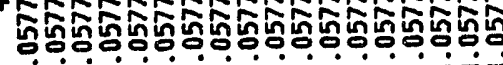

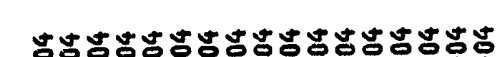

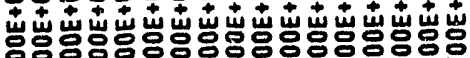

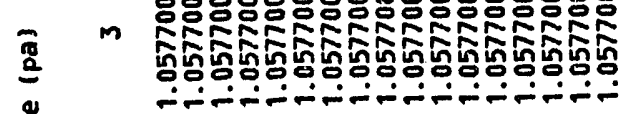

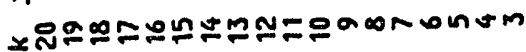

*

"

\&

㟧

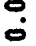

崖

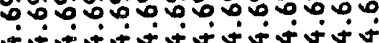
$=$

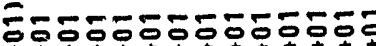

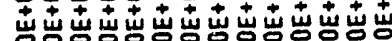

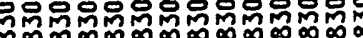

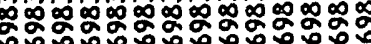

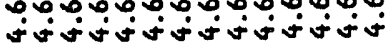
$=$

$\sim$

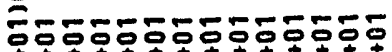

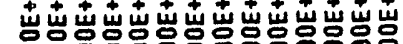

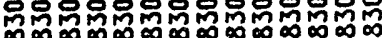

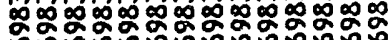
等

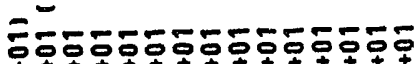

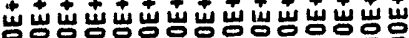

○.

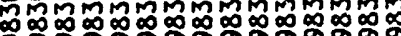
600

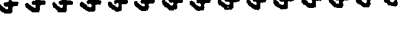

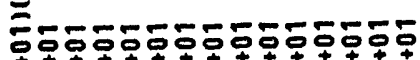

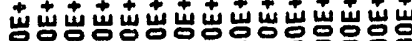
in

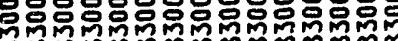
\%

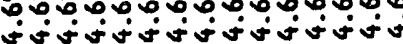

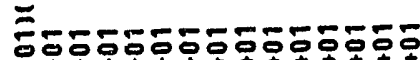

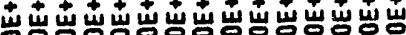
$\checkmark$

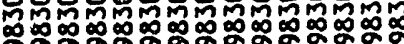

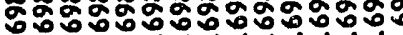

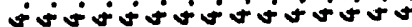

-

m

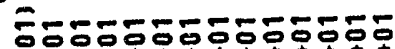

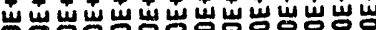

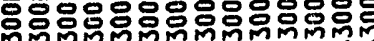
م0\%

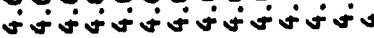

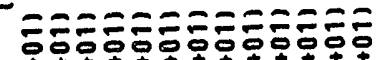

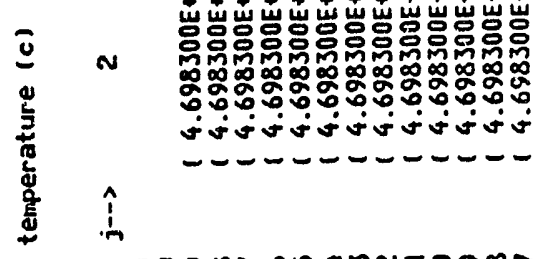



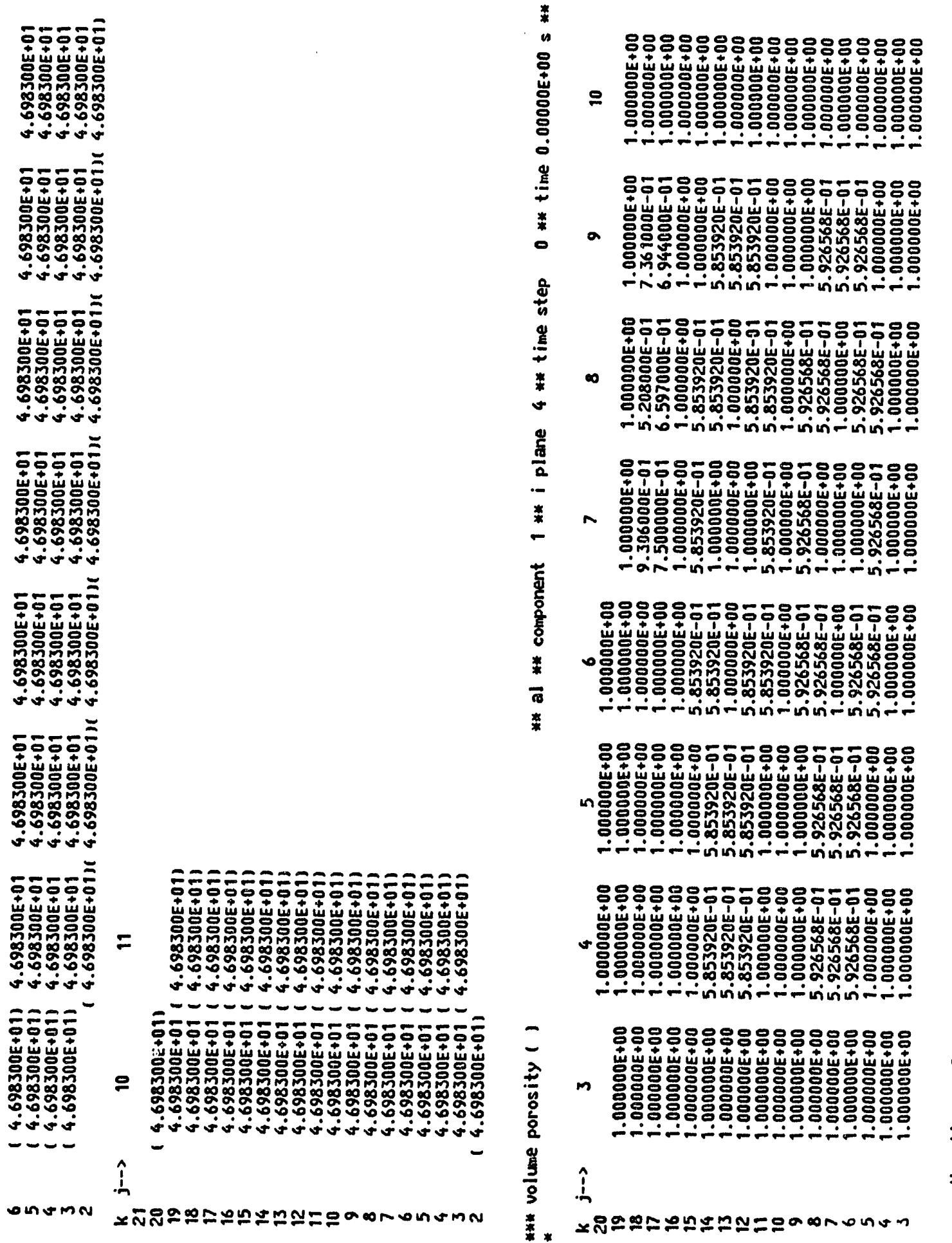

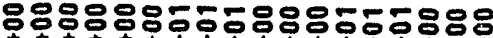

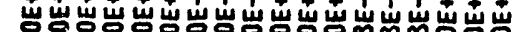

in

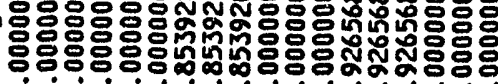

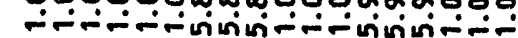
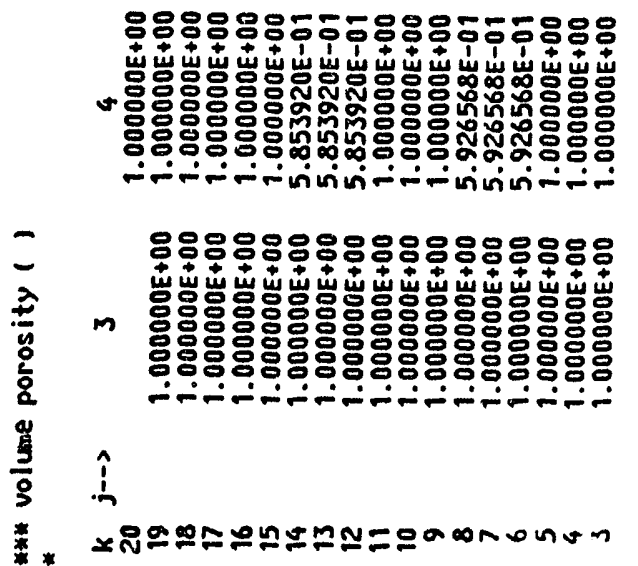

+
$\mathbf{5}$
8
8
8
0
$*$
2
2

$\underline{2}$

高 


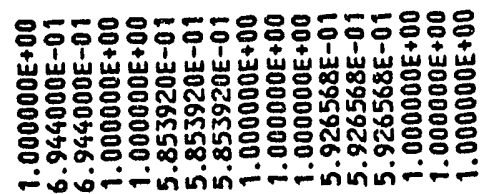

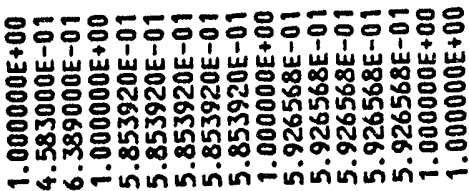

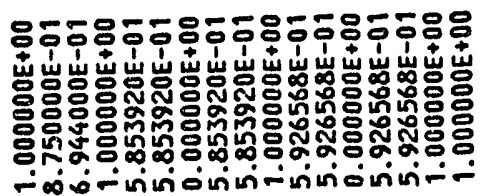

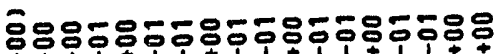

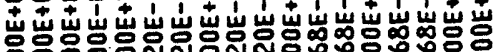

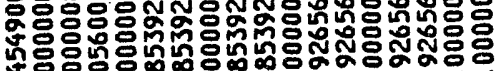
i-́-

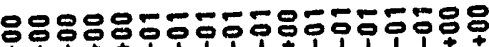

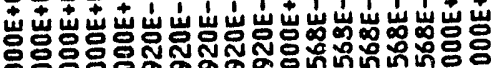

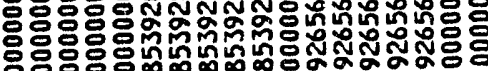

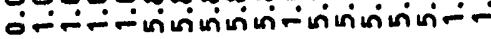

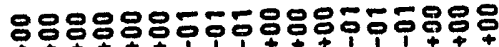

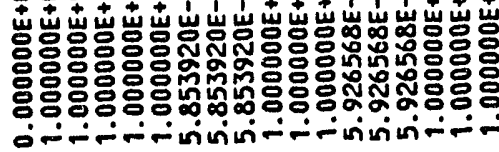

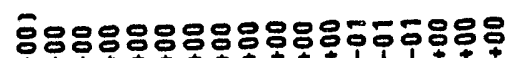

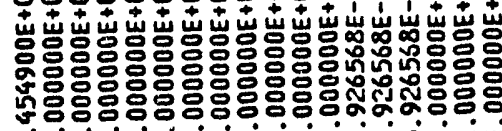
i-بن--

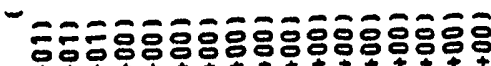

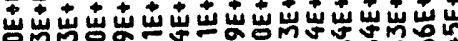

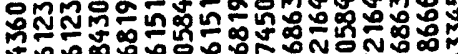

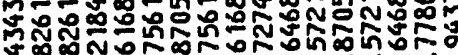
-ñ

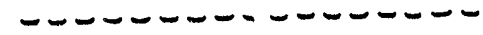

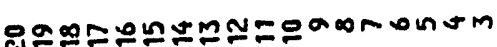

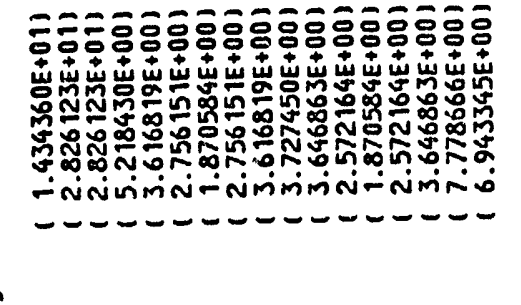

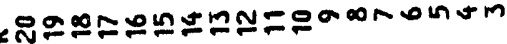

*

E.

*

$\circ$

gั

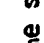

.

*

突

$\frac{\pi}{2}$

*

$-$

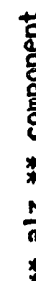

$n$

홍요용ㅇㅇㅇㅇㅇㅇㅇ

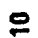

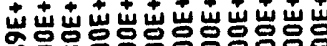

-

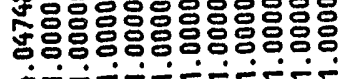

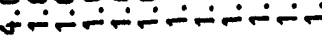

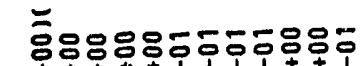

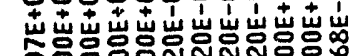

a

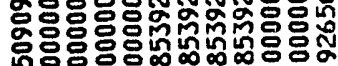
กุo =

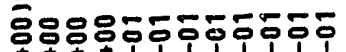

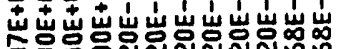

$\infty$

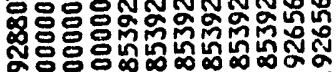

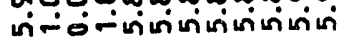
วิ

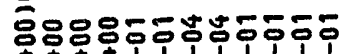

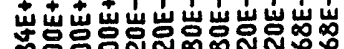

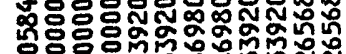

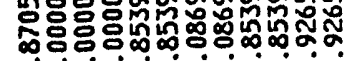

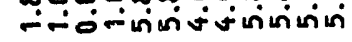

훙용ㅎㅎ후훟후후

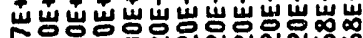

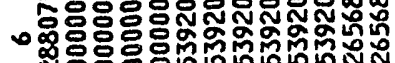

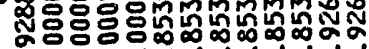
ที-- 五。

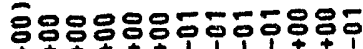

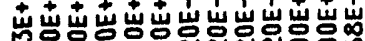
n

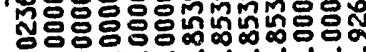
m--x-mininin-in כ 훙용두둥유

-

ב⿳亠口冋.

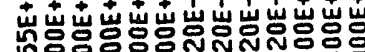

$\checkmark$

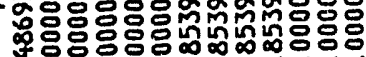
m-- "

m

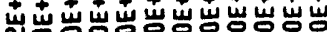

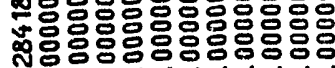

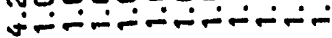
ín $-$

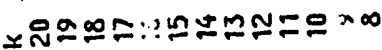




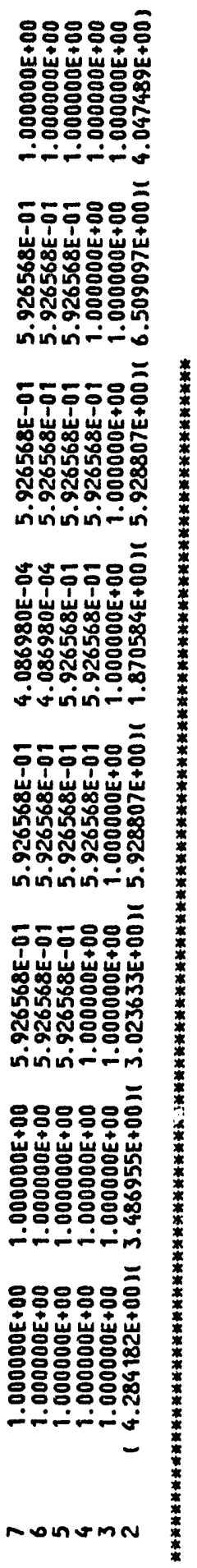



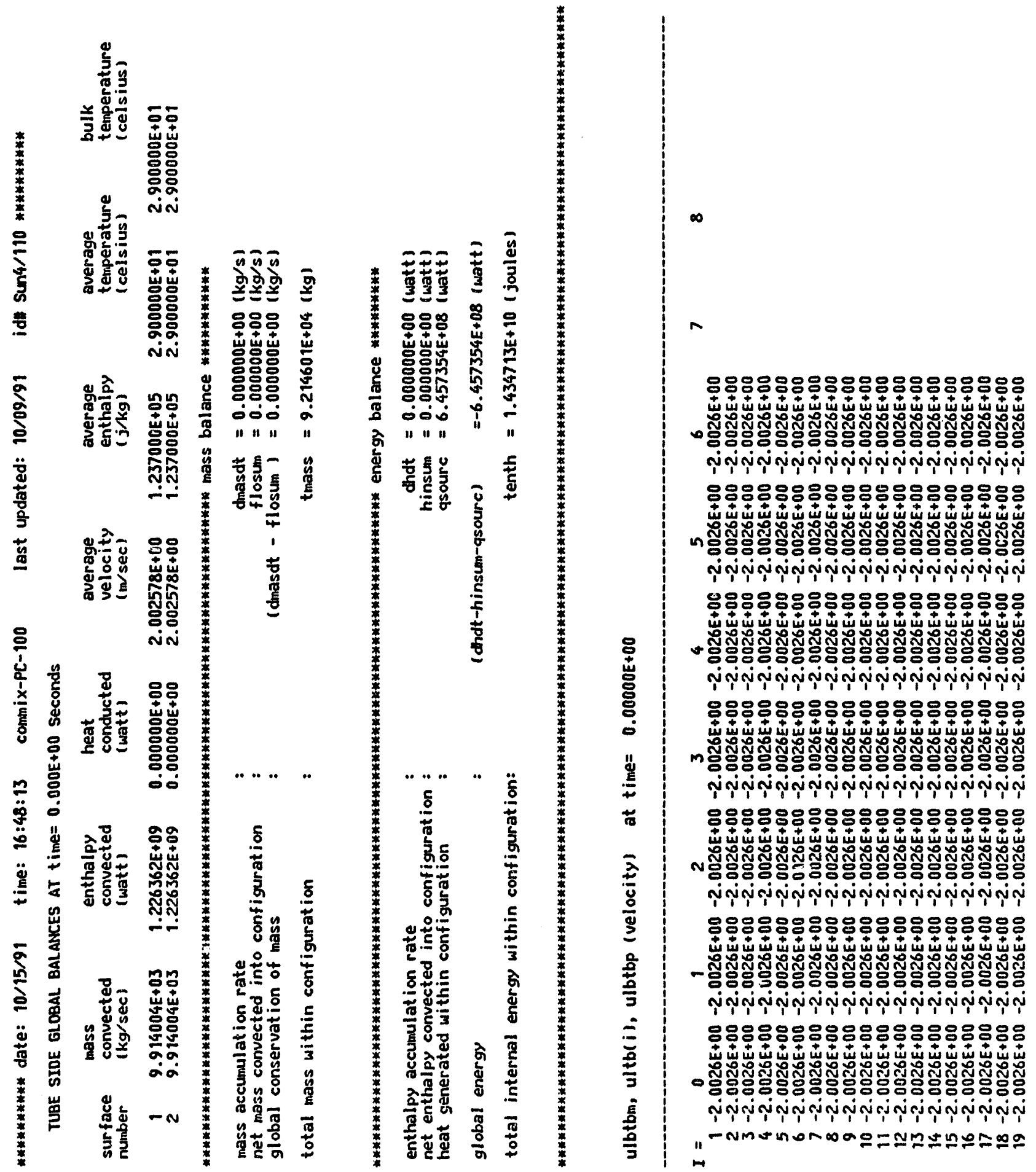


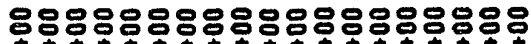

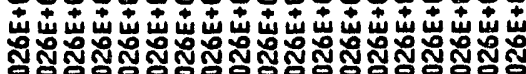

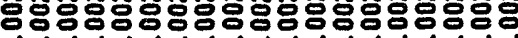

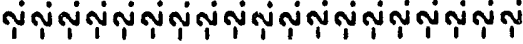

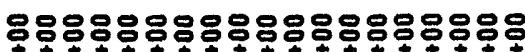

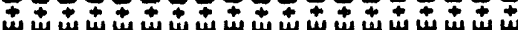

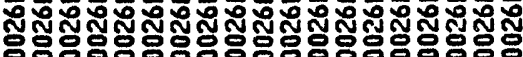

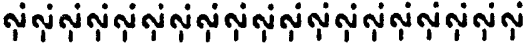

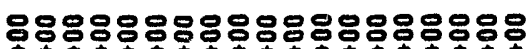

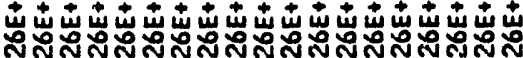

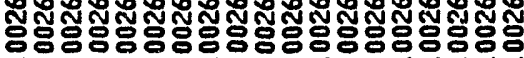

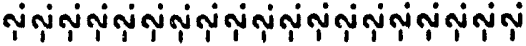

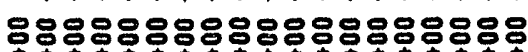

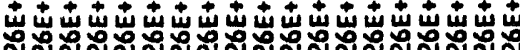

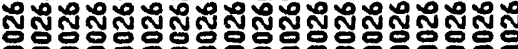
พñกำก

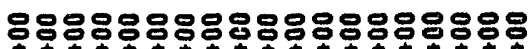

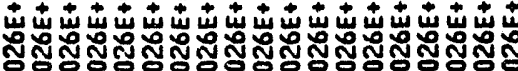

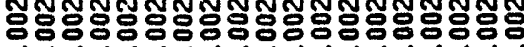

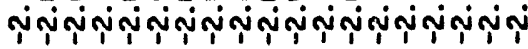

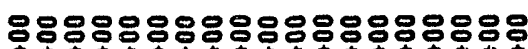

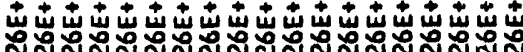

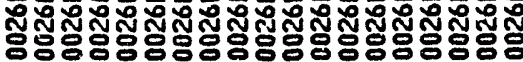
ҮิT

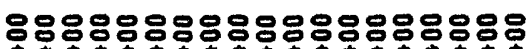

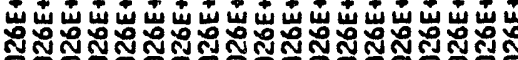

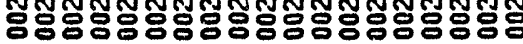

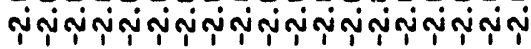

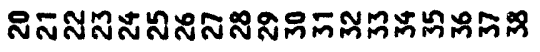

$\infty$

$n$

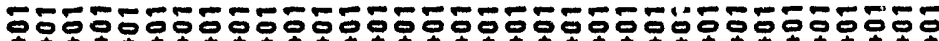

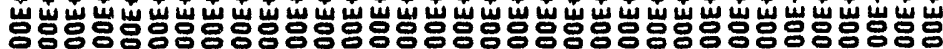

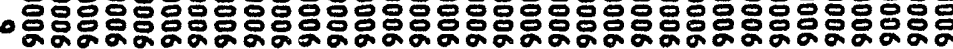

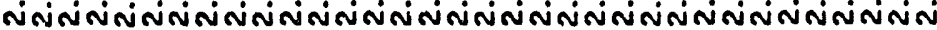

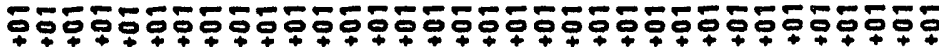

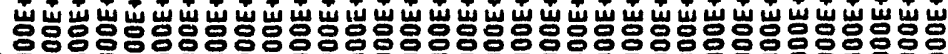

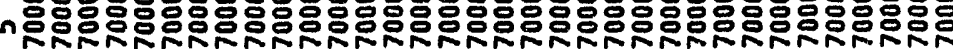

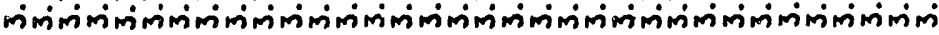

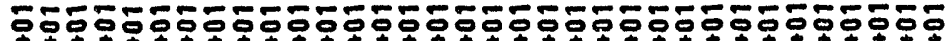

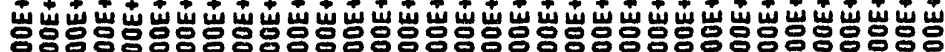

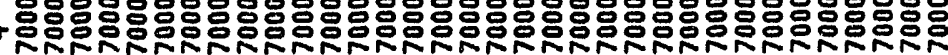

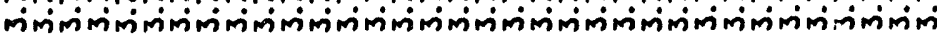

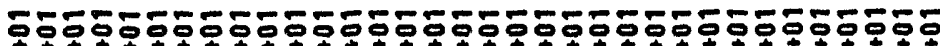

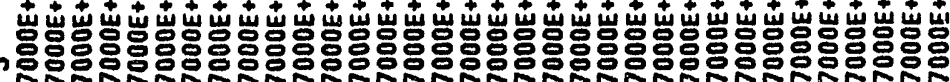

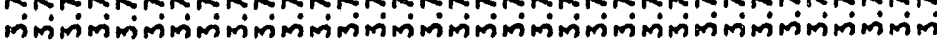

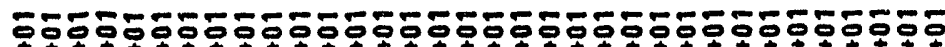

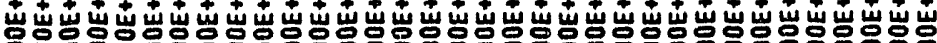

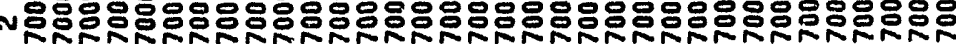

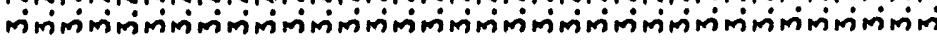

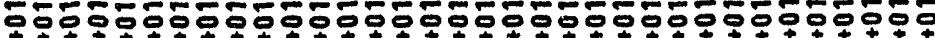

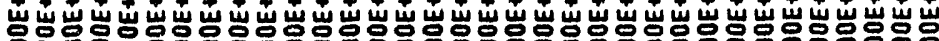

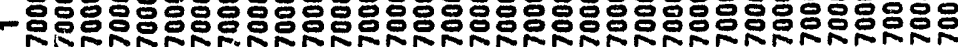

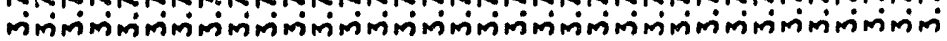

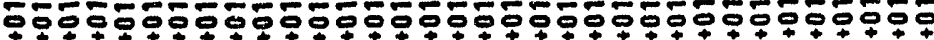

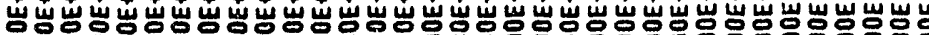

-

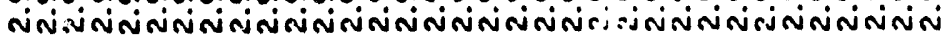
11 


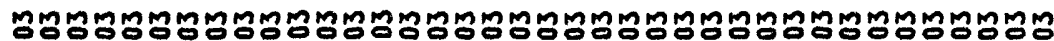

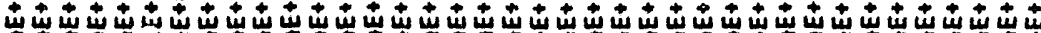

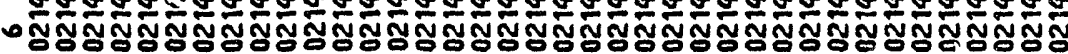
ininin

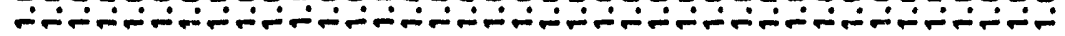

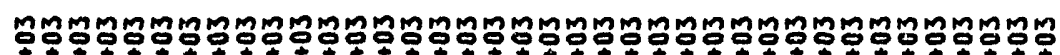

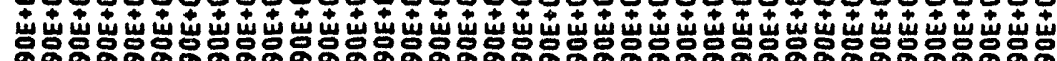
n

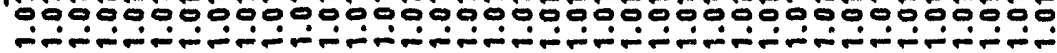

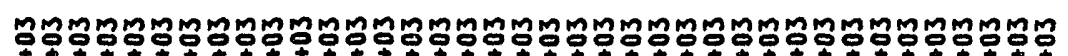

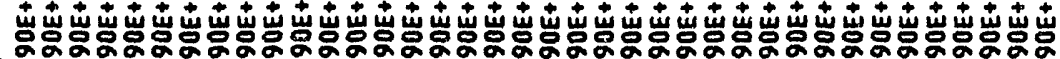

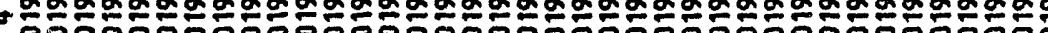
-

5050

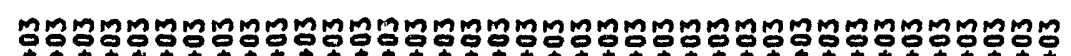

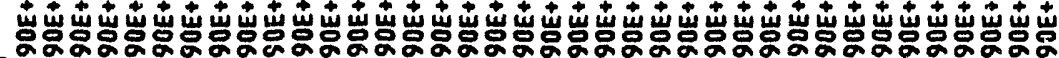

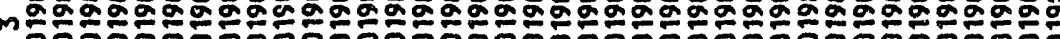
-

5050

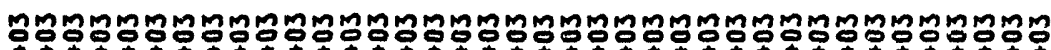

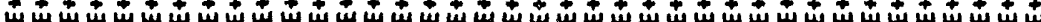

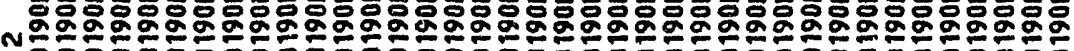

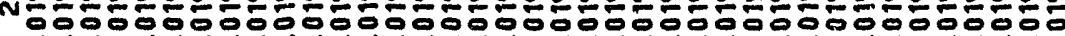

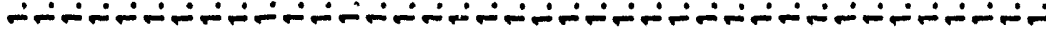

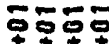

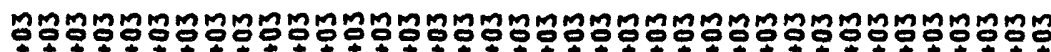

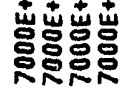

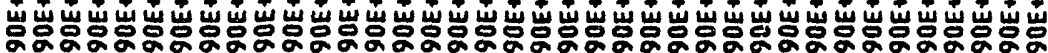
$00000000000000000000000000000000=00000$ minimin

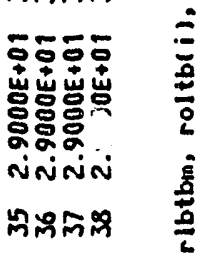

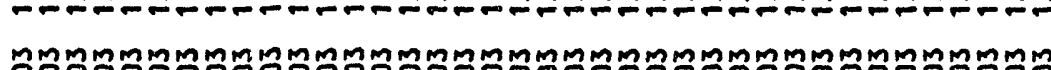

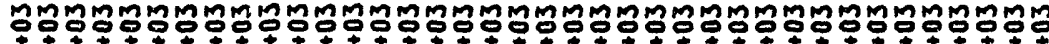

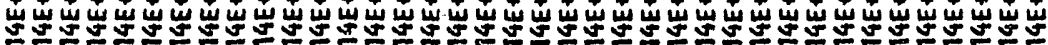

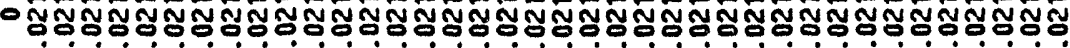

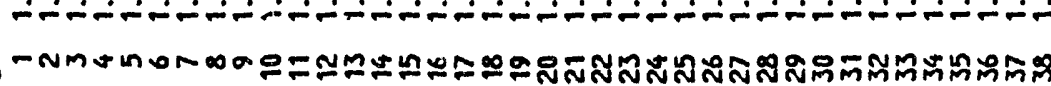
$n$

-

1 NMs 


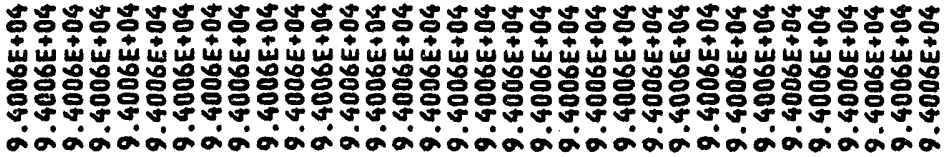

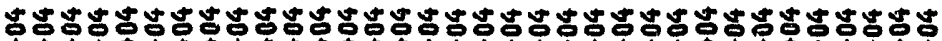

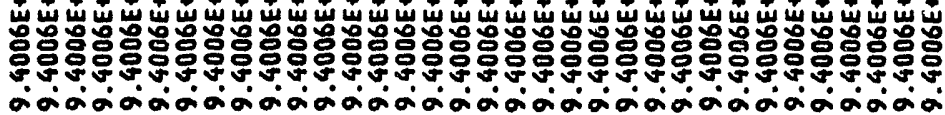

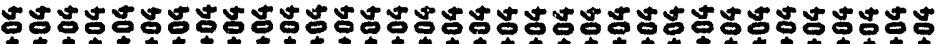

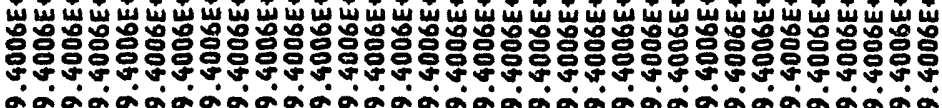

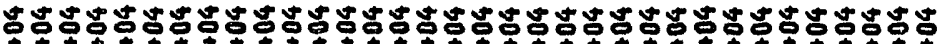

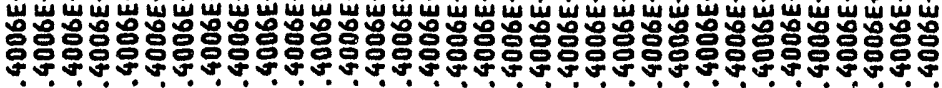

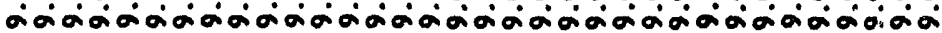

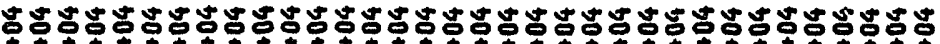

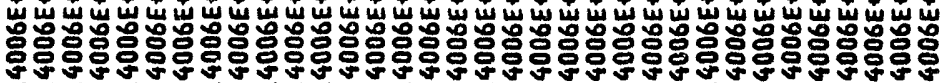

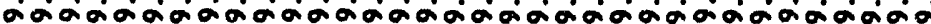

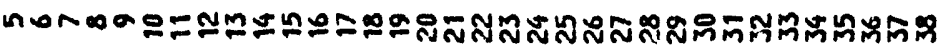

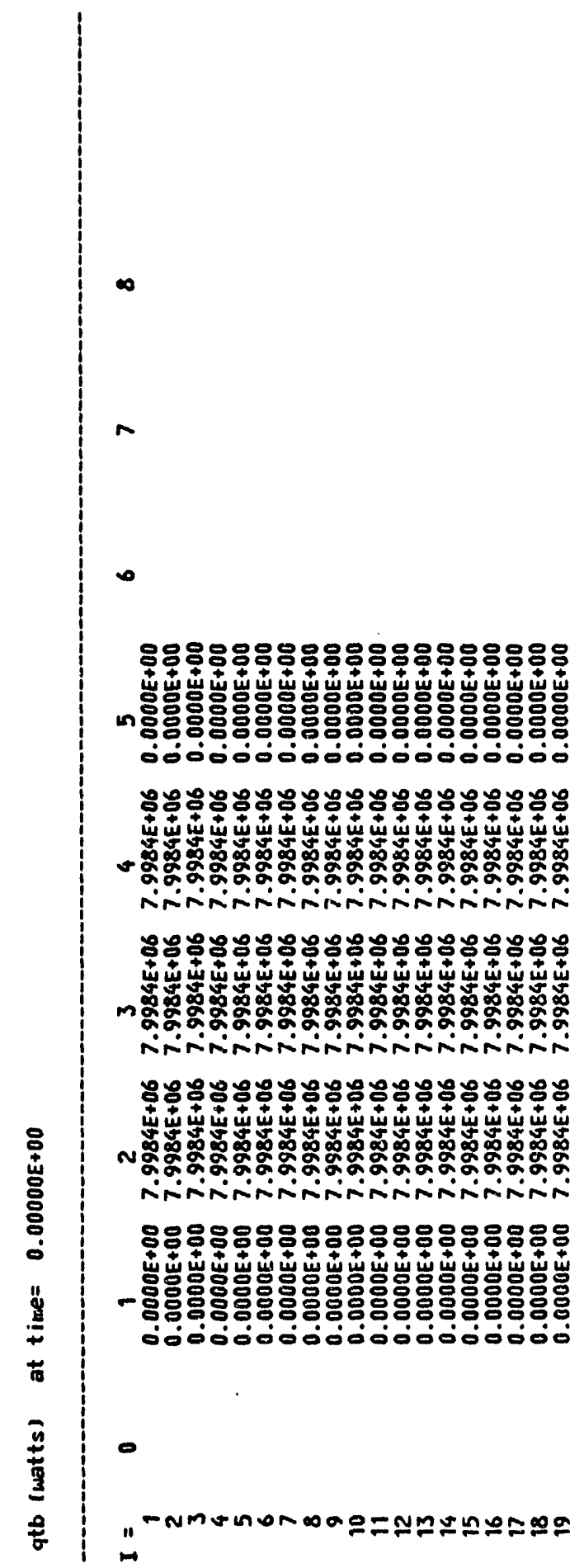




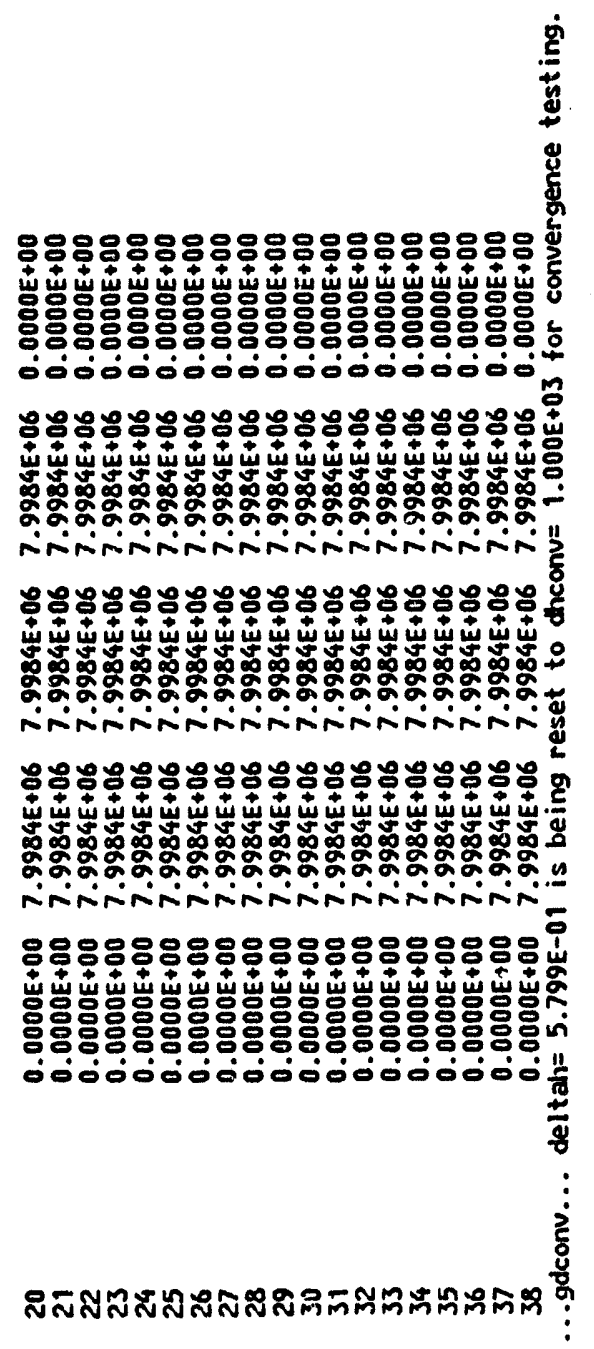




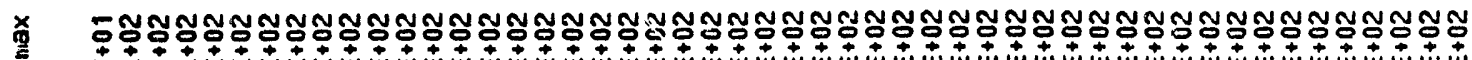

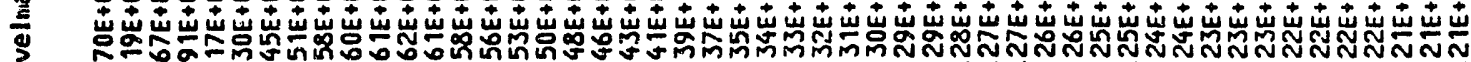

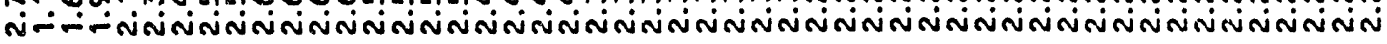

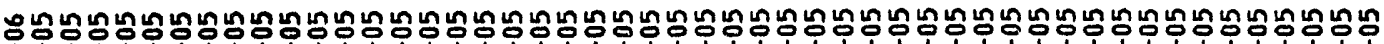

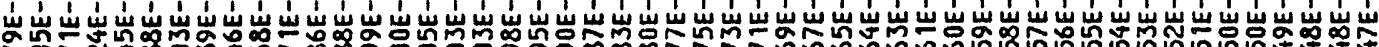

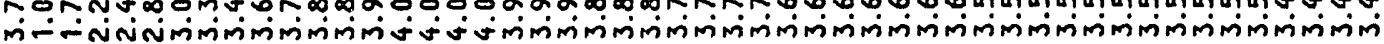

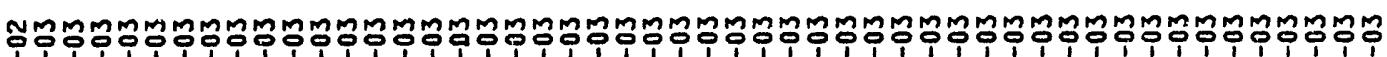

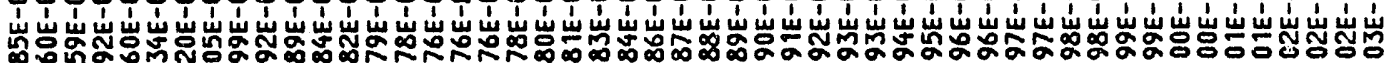

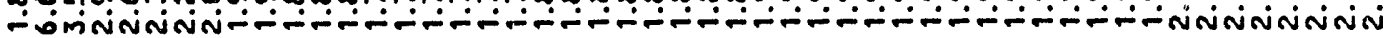

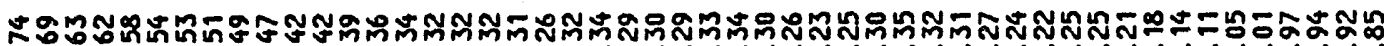

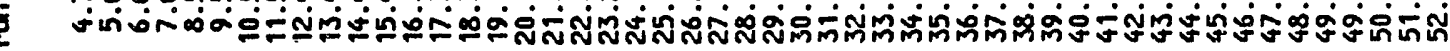

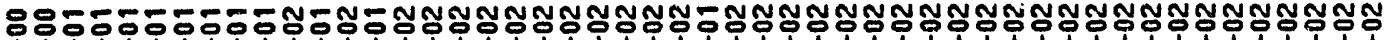

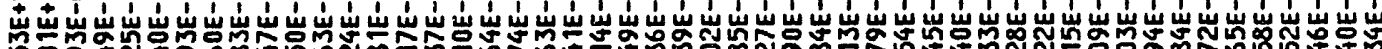

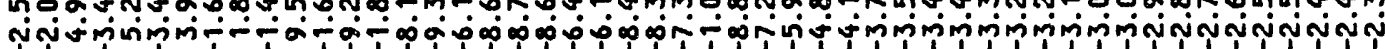

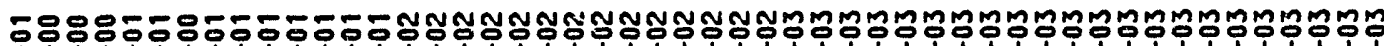

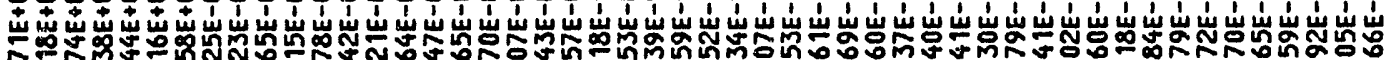

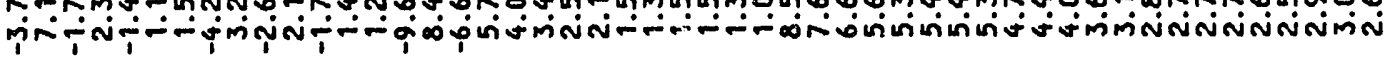

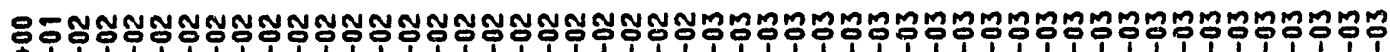

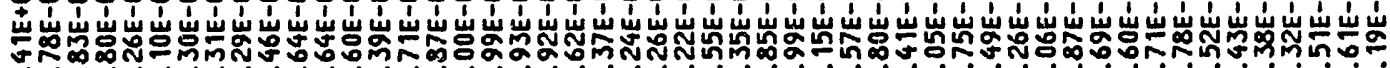

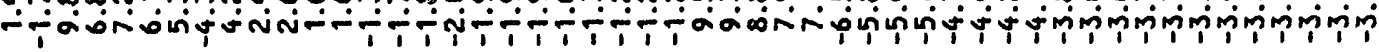

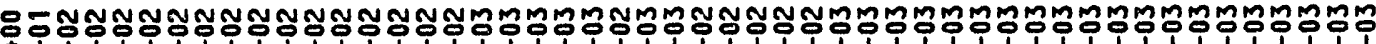

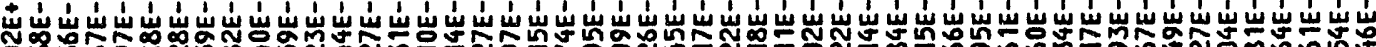
ง

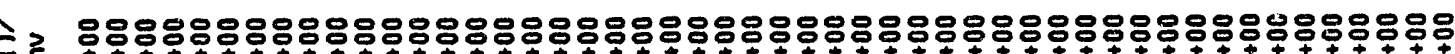

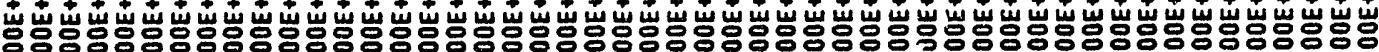

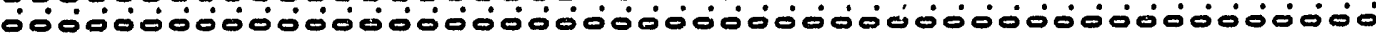

$\pm$

作

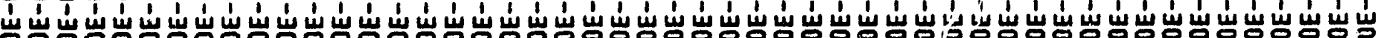

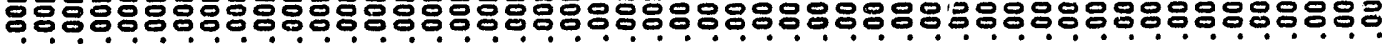

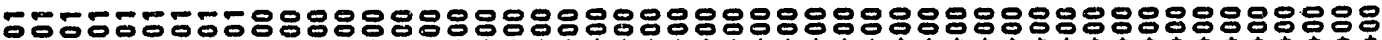

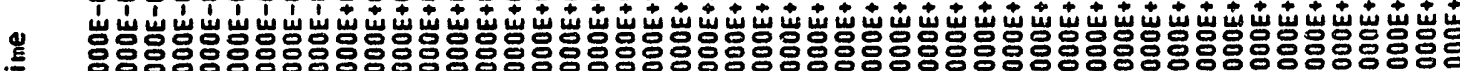

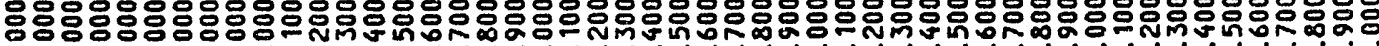

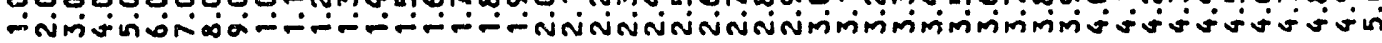

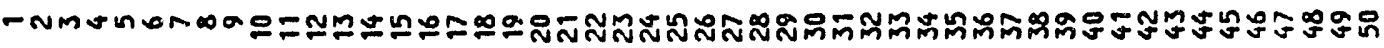




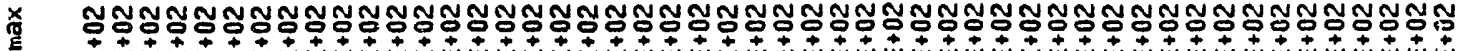

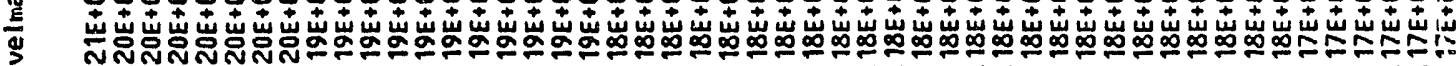

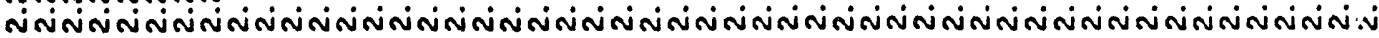

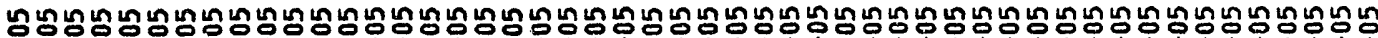

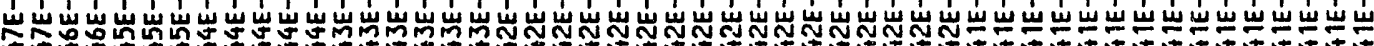

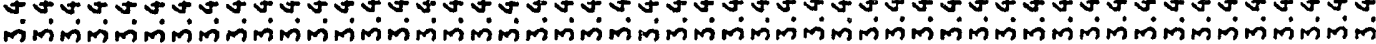

薄

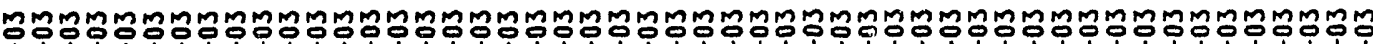

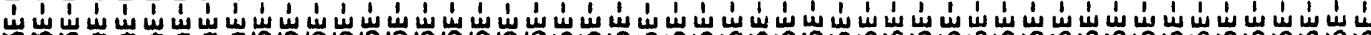

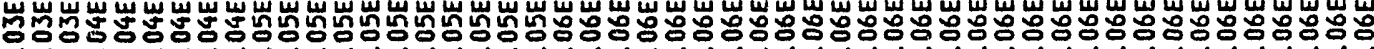

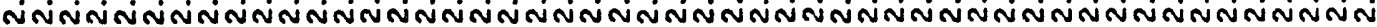

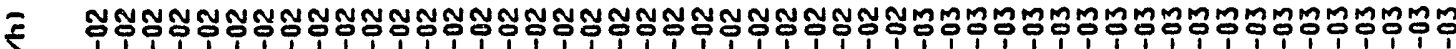

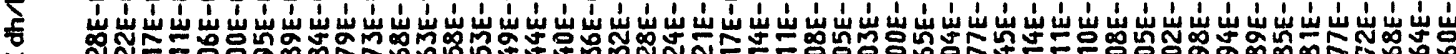

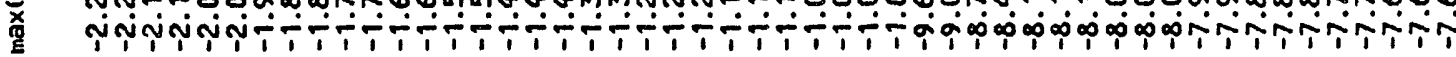

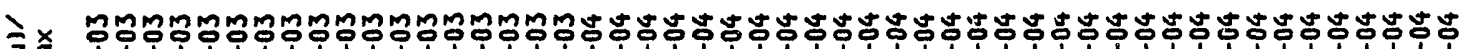

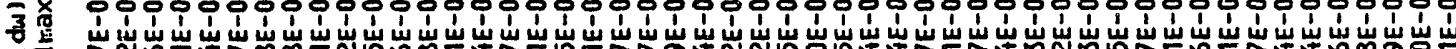

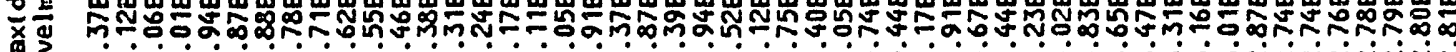

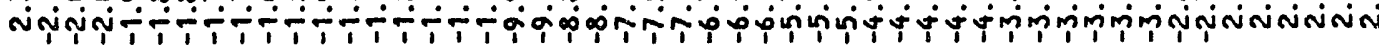

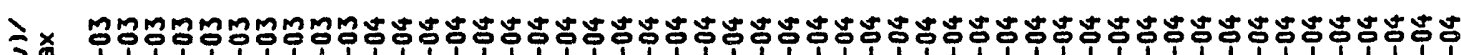

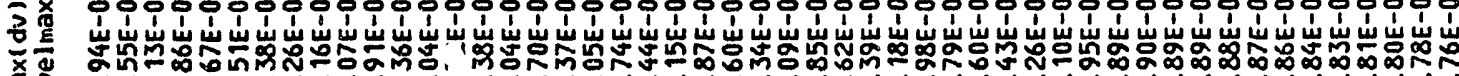
NNN

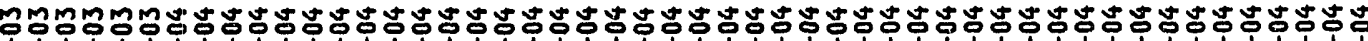

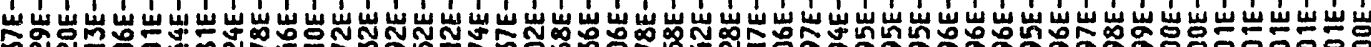
m

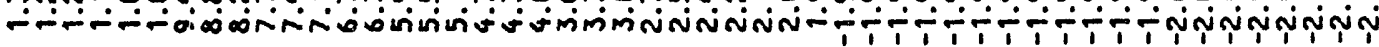

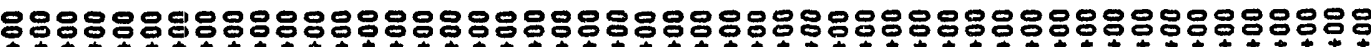

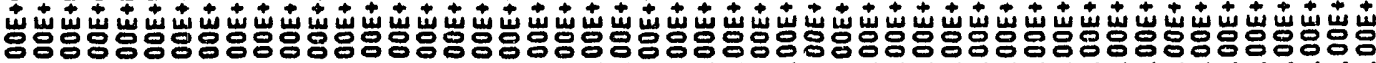

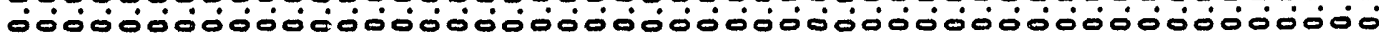

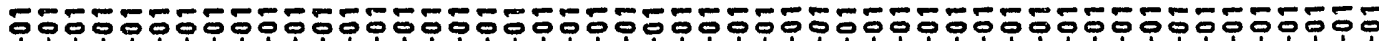

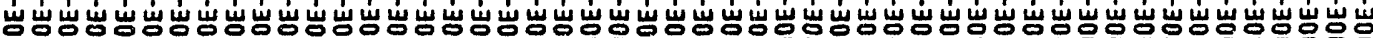
о一்

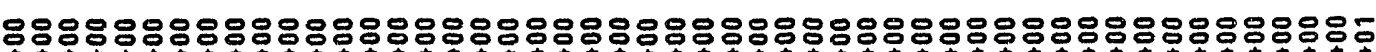

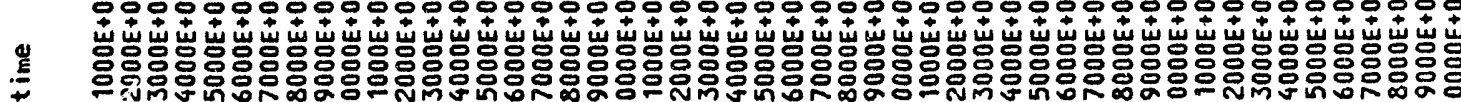

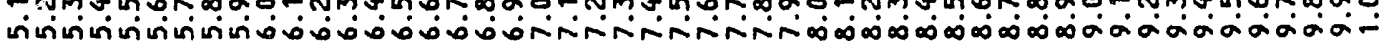

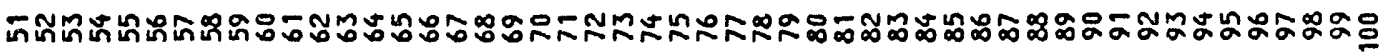


E

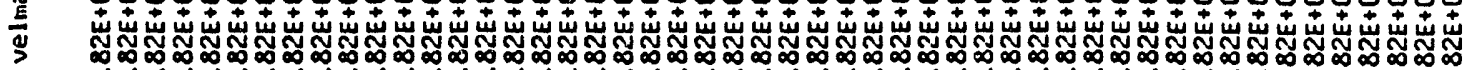

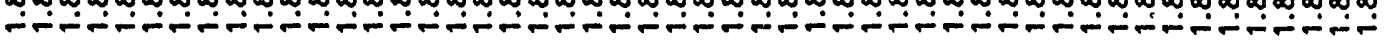

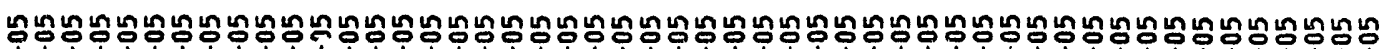

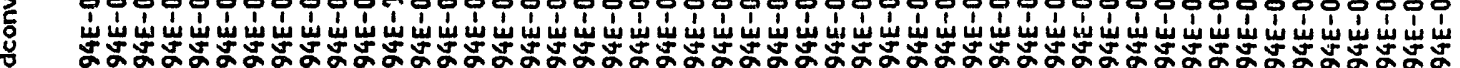

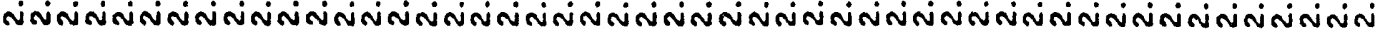

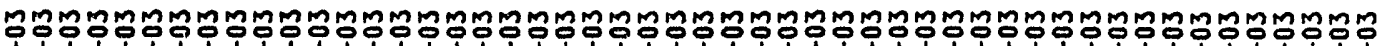

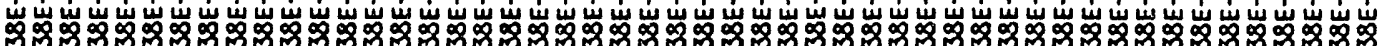

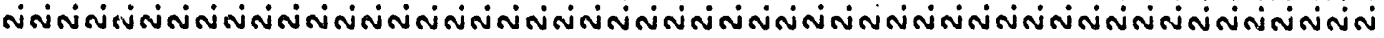

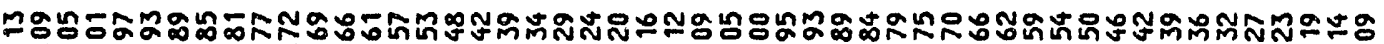

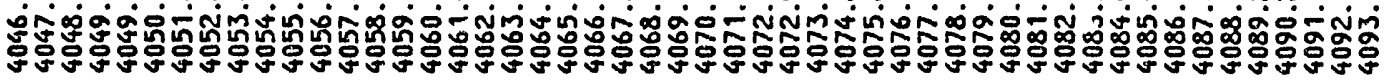

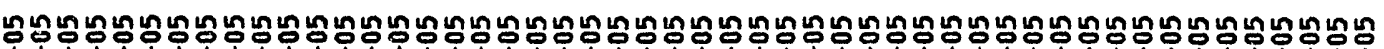

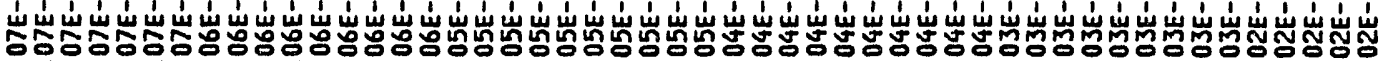

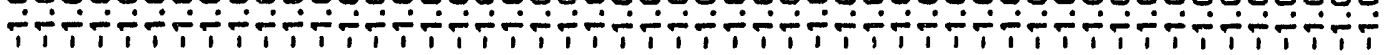

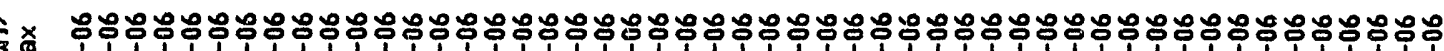
సึ心

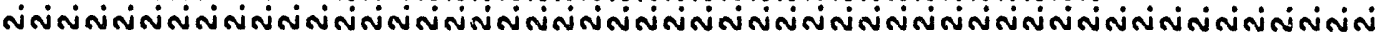

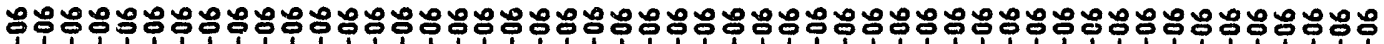

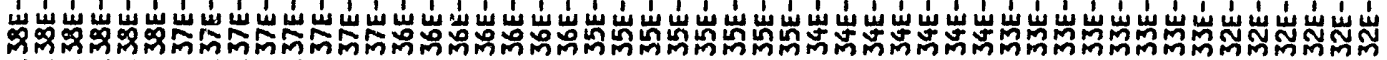

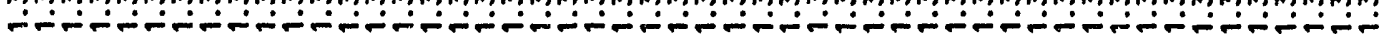

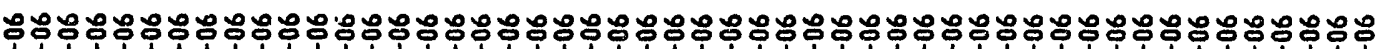

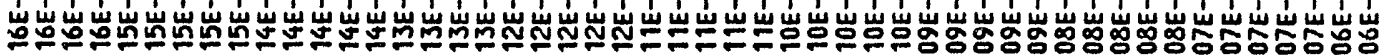

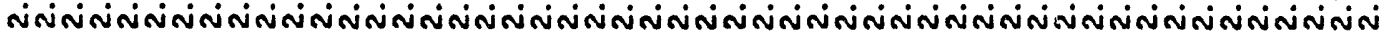

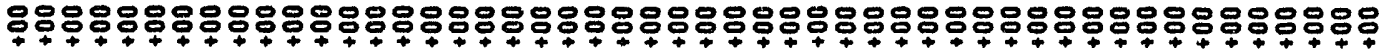

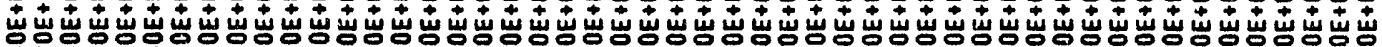

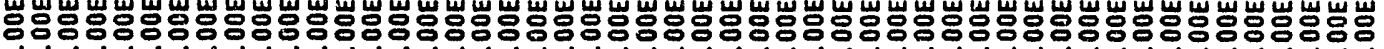

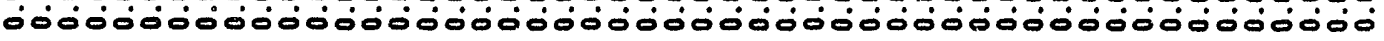

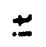

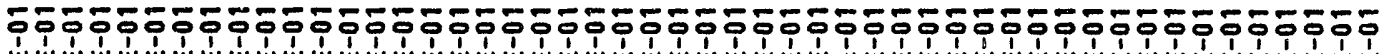

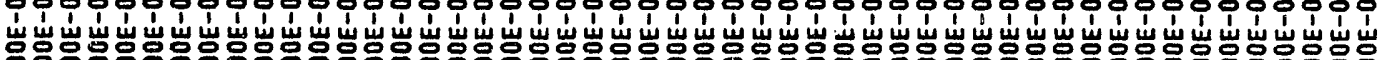

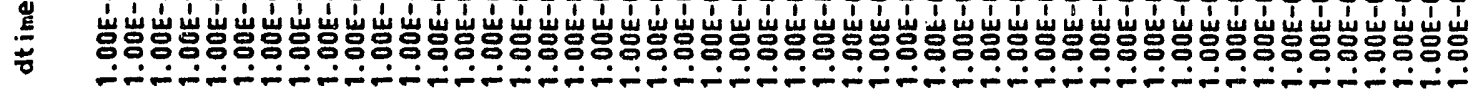

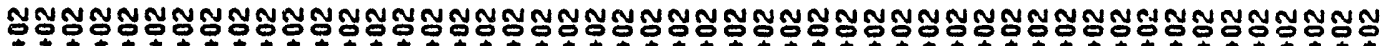

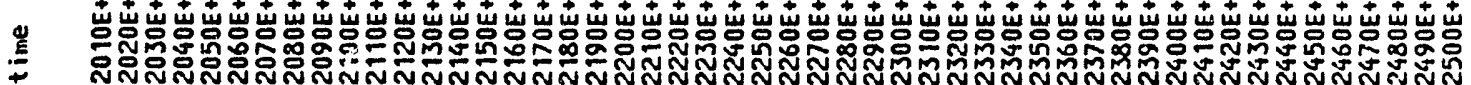

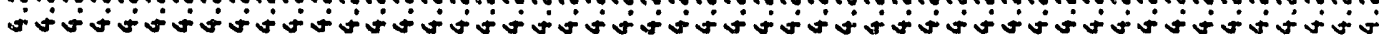

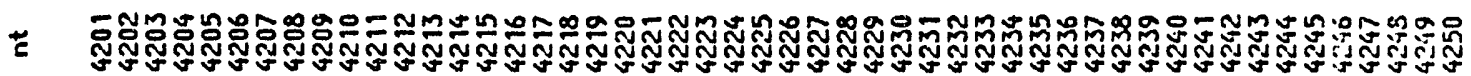



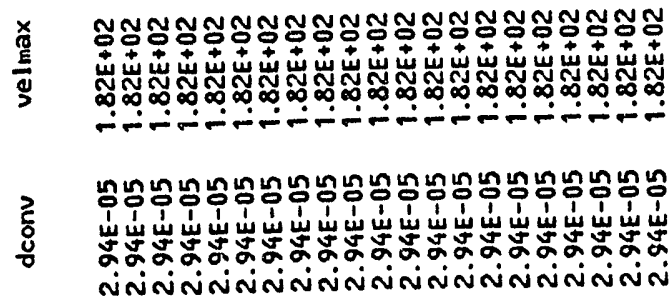

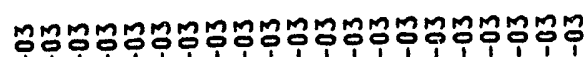

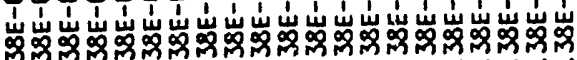

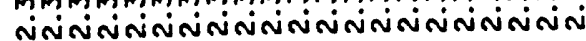

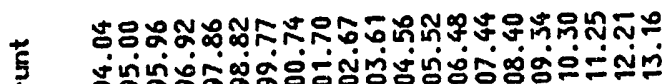

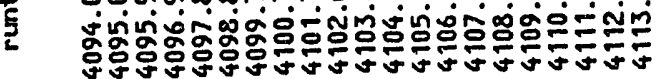

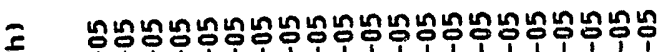

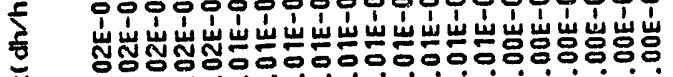

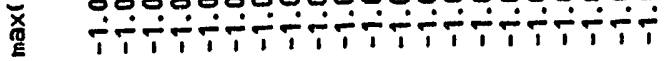

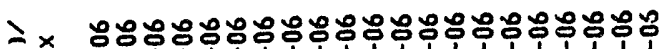

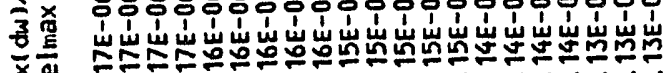

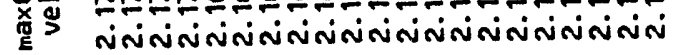

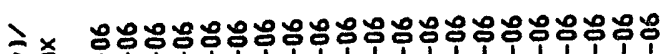

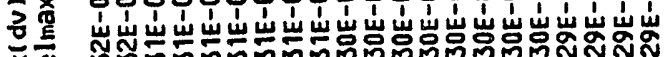

希

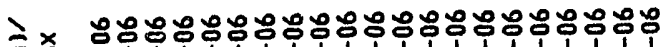

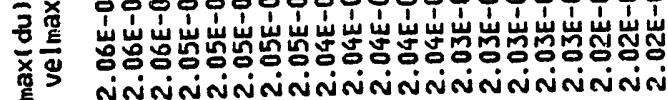

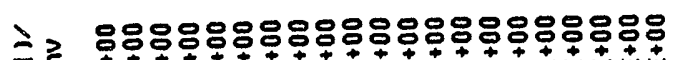

₹

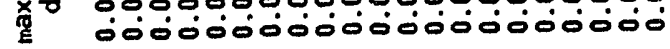

$\pm$

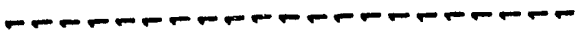

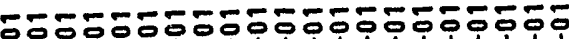

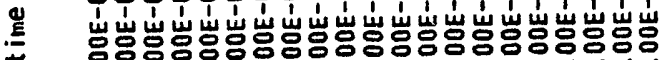

苦 0090000000

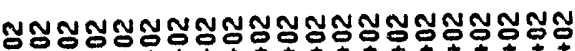

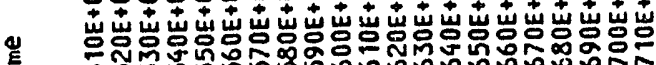

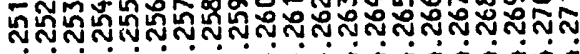

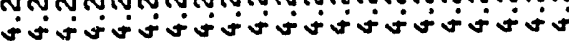

$\underline{E}$

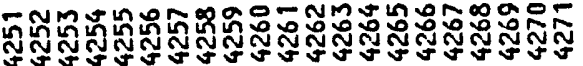

$\mp \frac{\infty}{80}$

号

กิ้

สูู

* * *

䊁数蒌

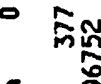

음

$\overline{7}$

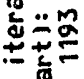

焉若

ํํㅇำํํำ

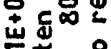

ㅎำ

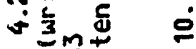

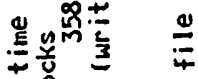

$+\frac{0}{2}+\frac{1}{2}$

ฐ

寝

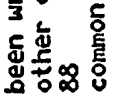

范苍

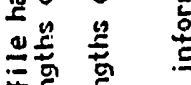

प हो

焉站 

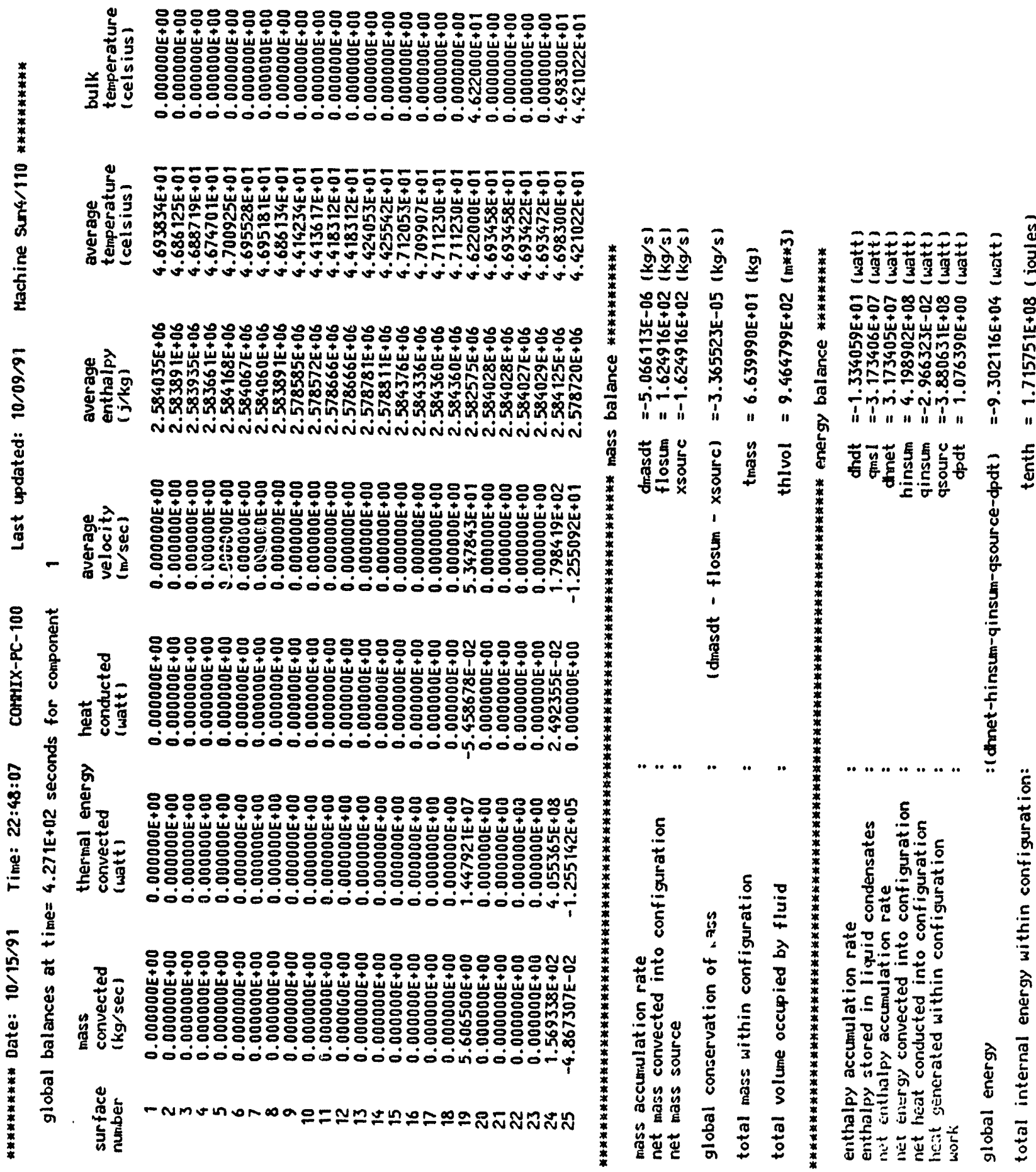


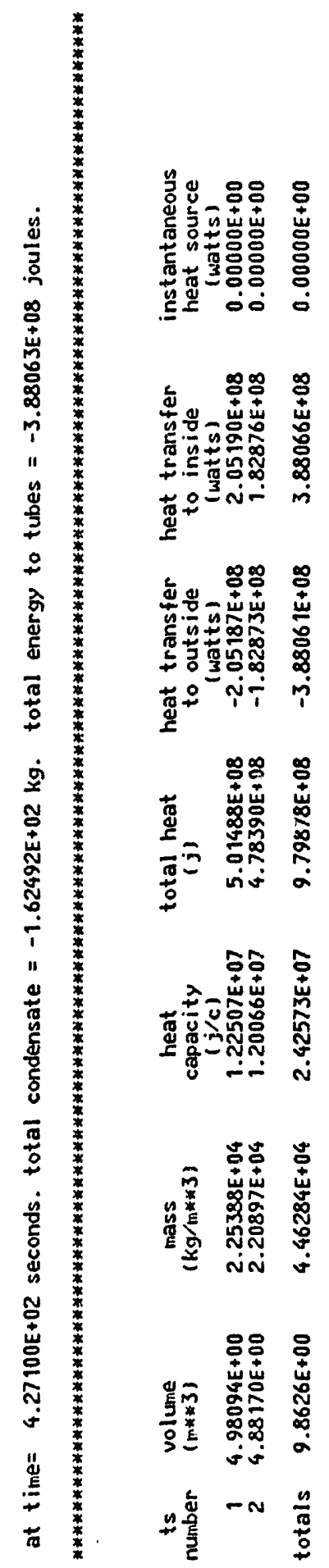



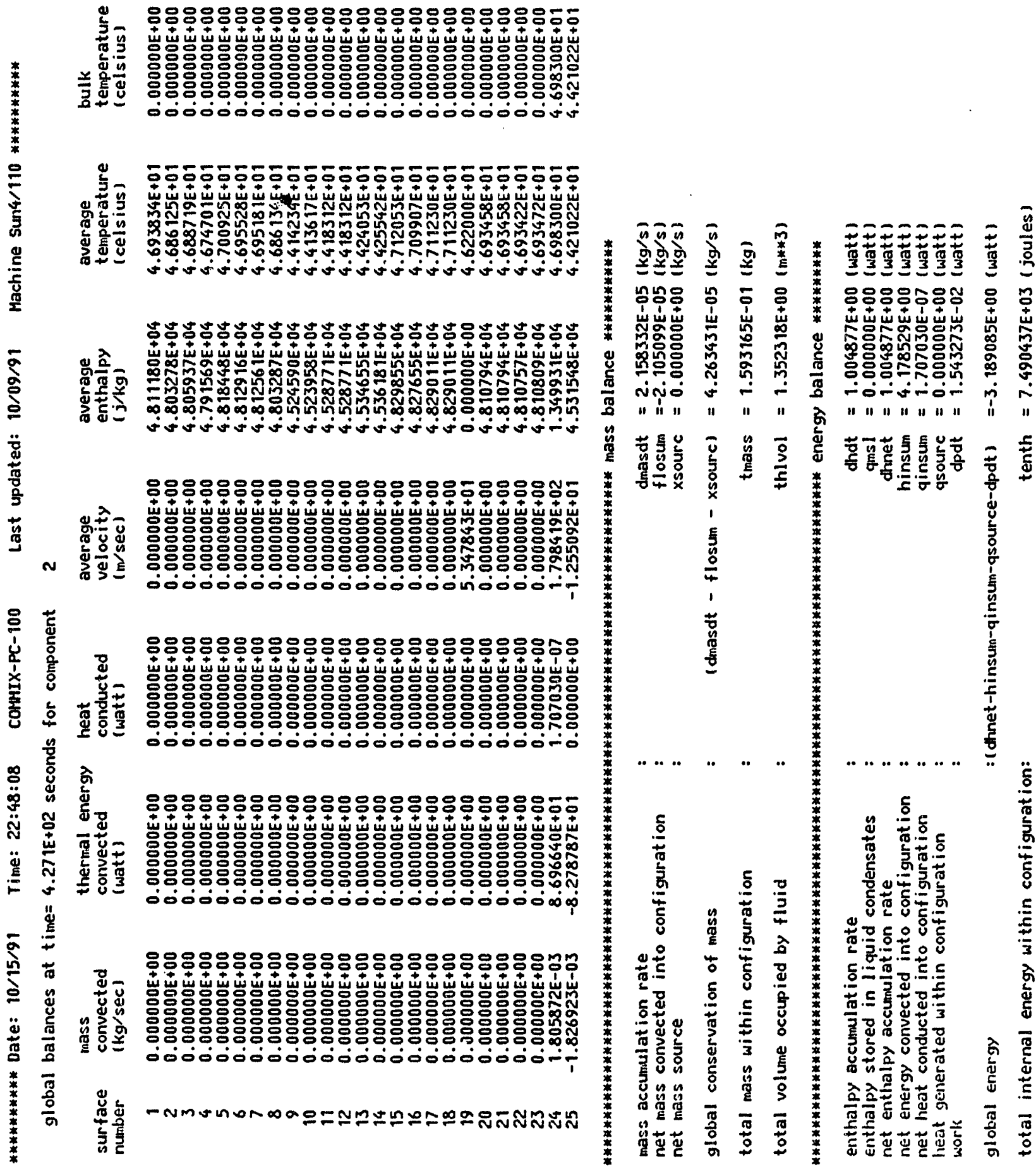


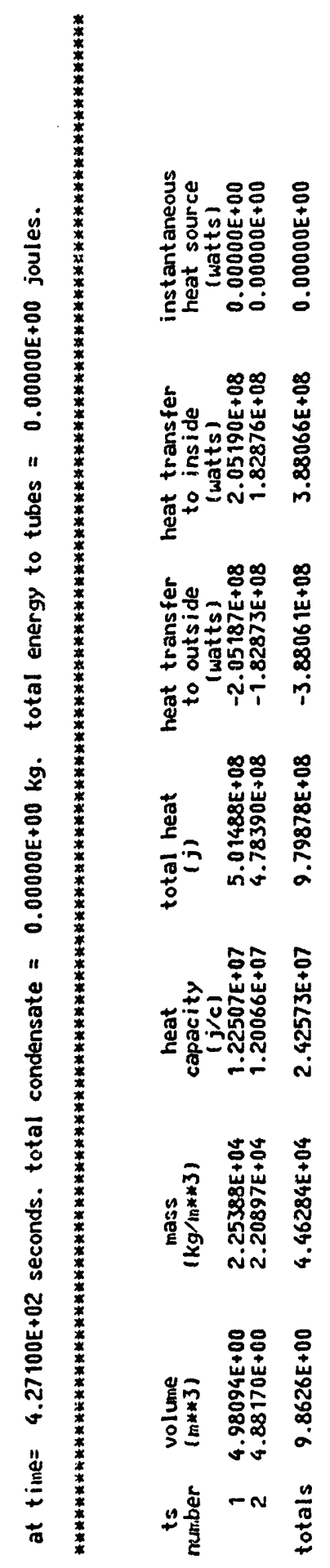



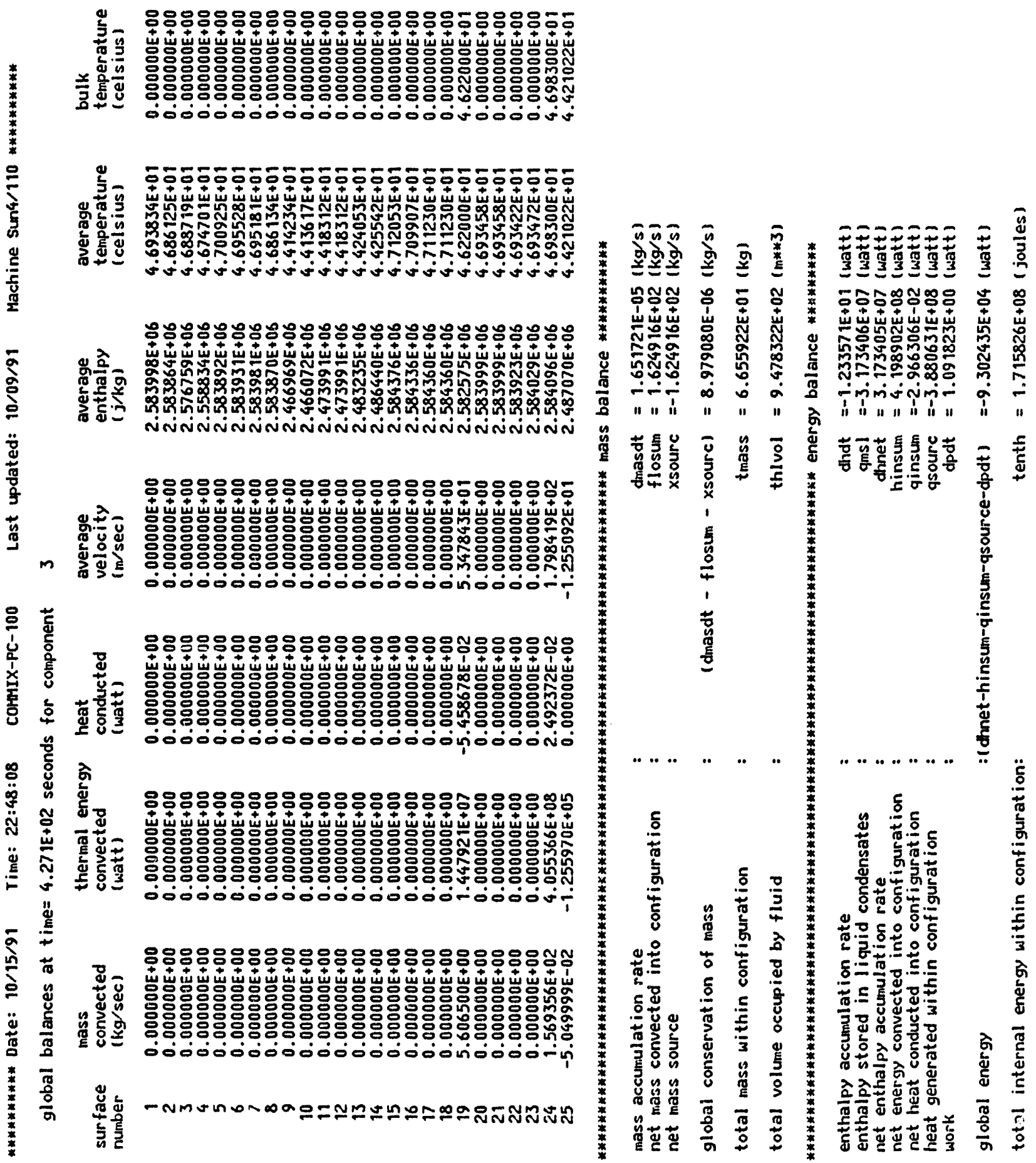


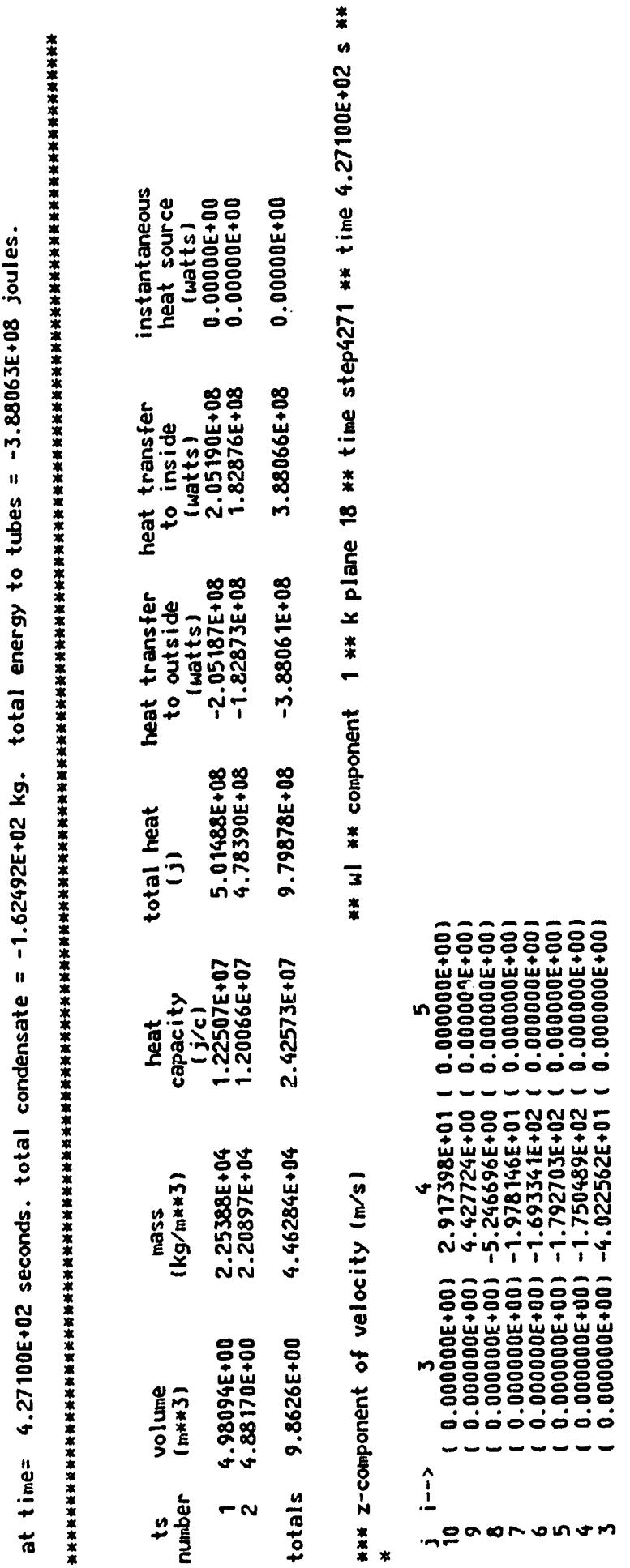

웅

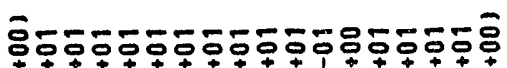

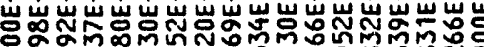

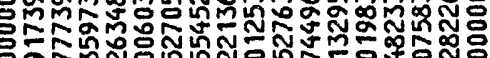

onimivinipino-ño

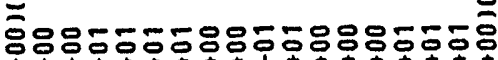

a

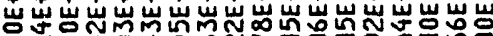

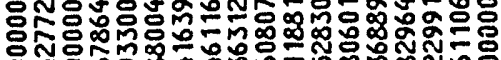

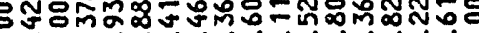

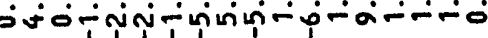

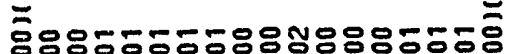

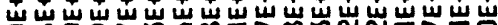

$\infty$

管

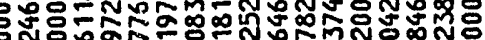

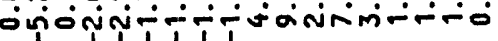

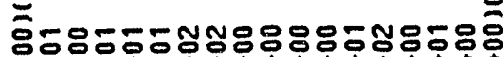

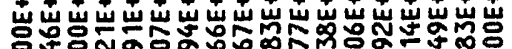

N

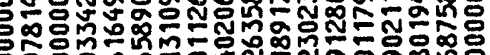

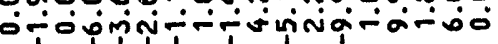

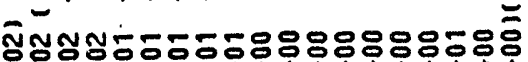

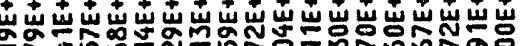

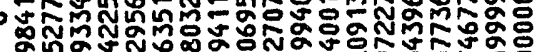

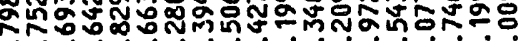

१т-

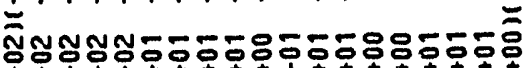

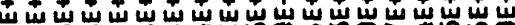

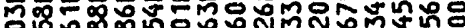

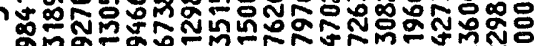

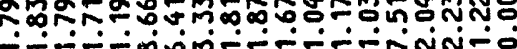

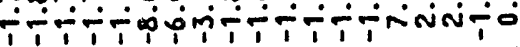

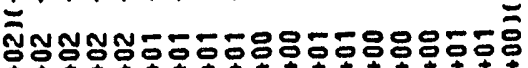

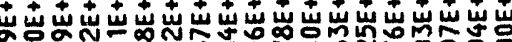

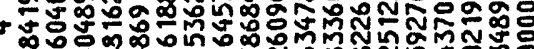

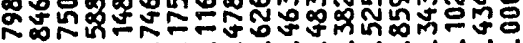

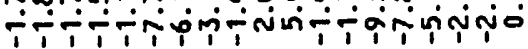

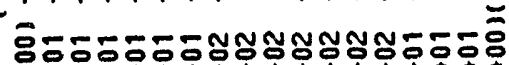

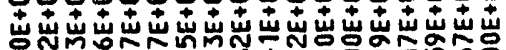

m

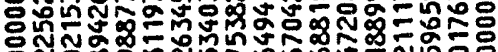

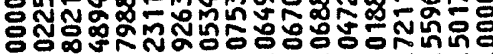

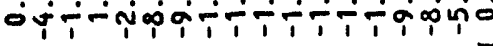<smiles>[CH][AlH]</smiles> 


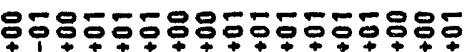

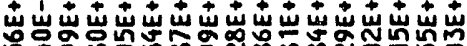

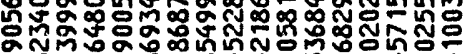

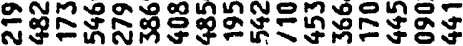

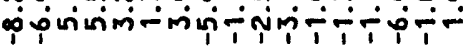

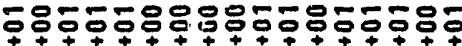

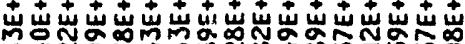

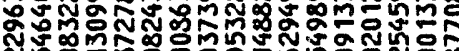

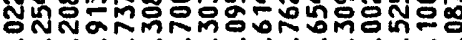

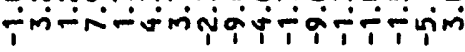

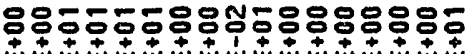

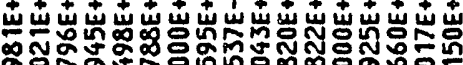

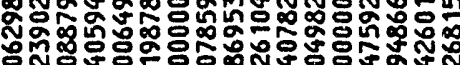

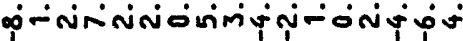

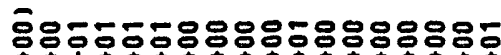

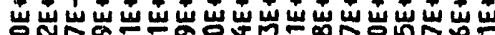

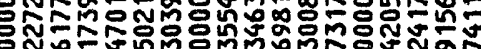

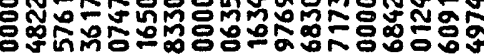

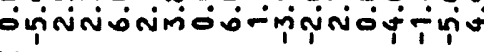

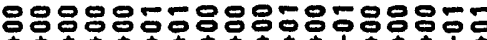

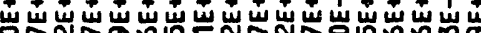
ธิ์สํํำ

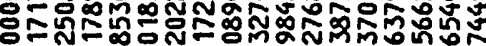

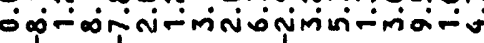

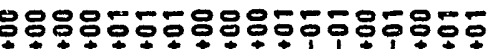

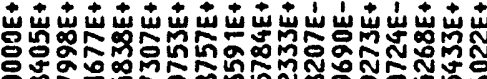

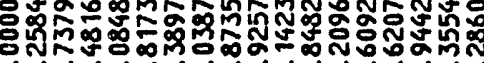

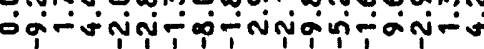

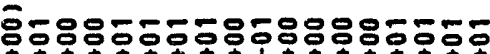

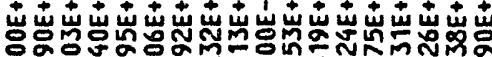
ธั口ำ

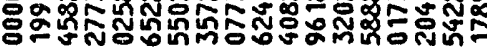

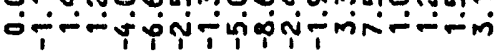

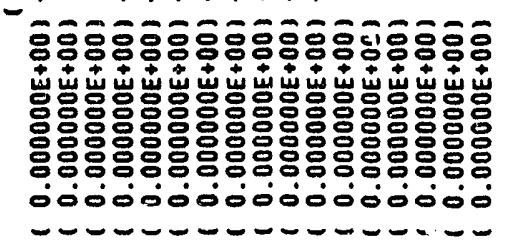

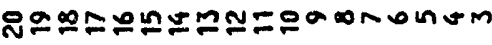

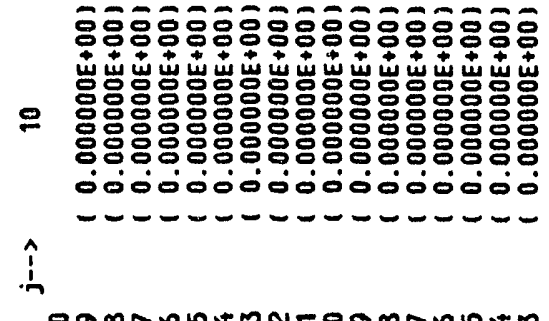

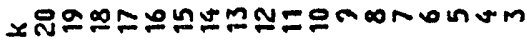

(3)

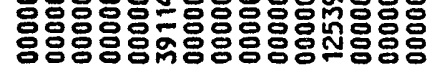
00000000000000

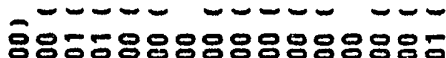

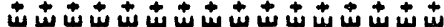

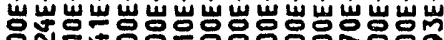

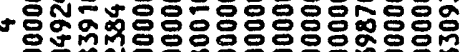

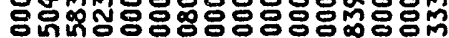

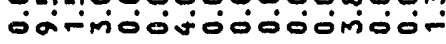
$=$

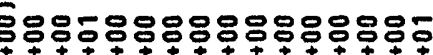

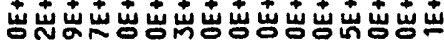

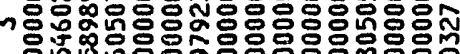

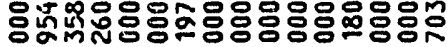

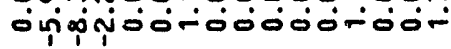

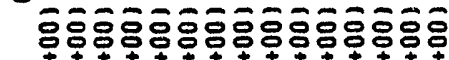

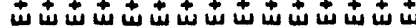

N

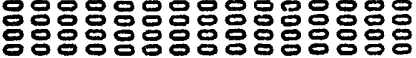
0000000000000 - - - - - - - - $\hat{\imath}$

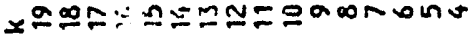




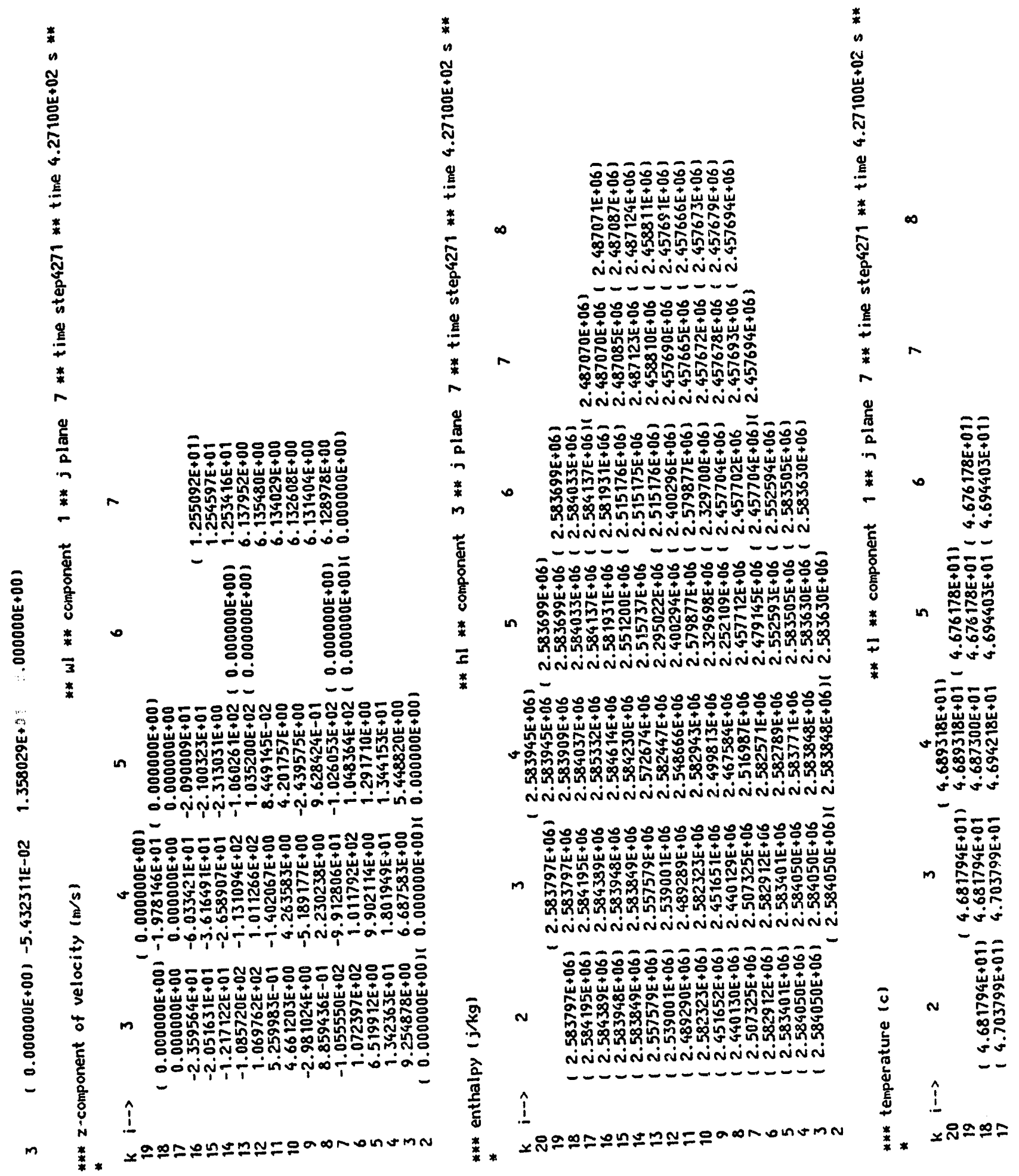




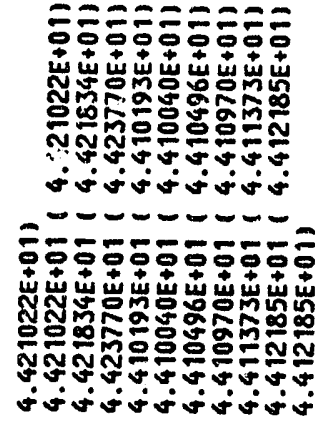

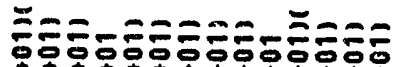

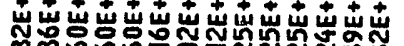

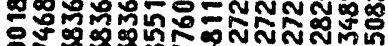

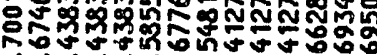

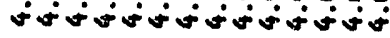

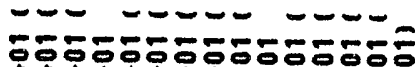

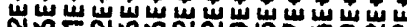

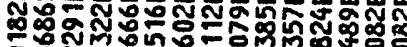

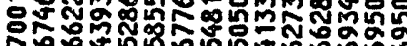

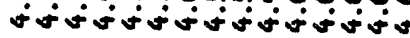

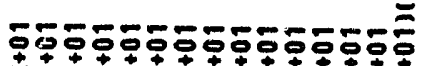

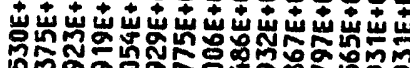

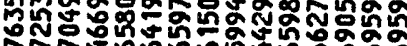

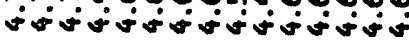

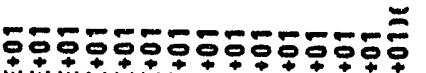

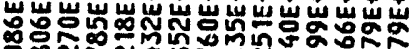

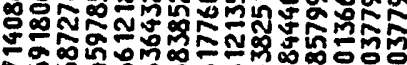

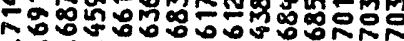

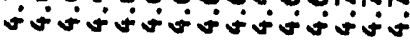

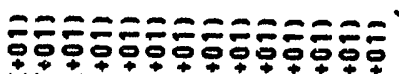

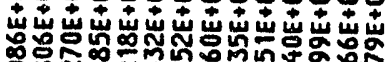

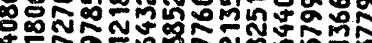

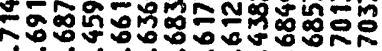

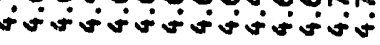

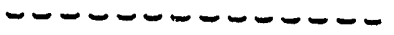

ำエณッニ

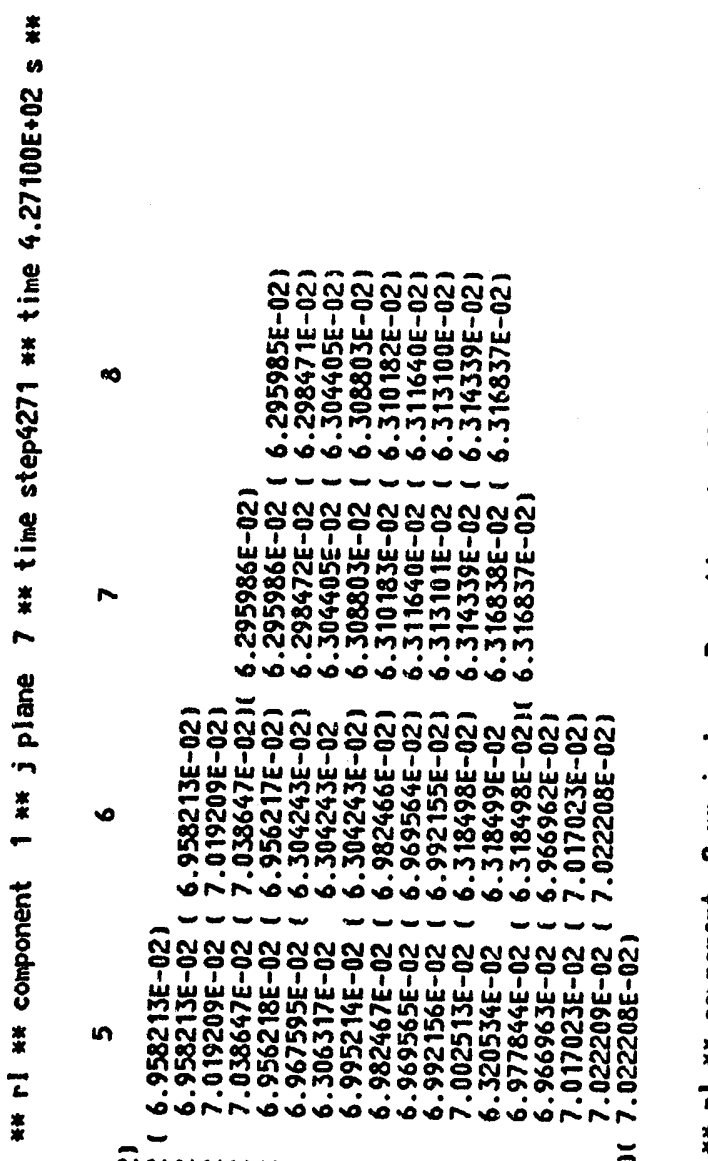

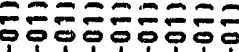

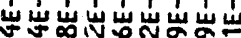

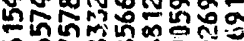

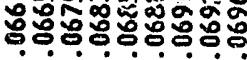

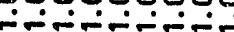

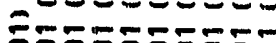

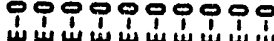

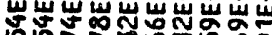

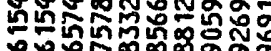

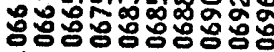

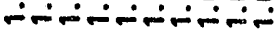

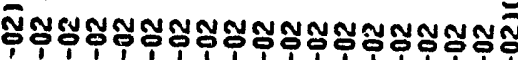

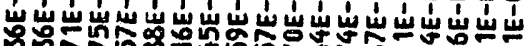

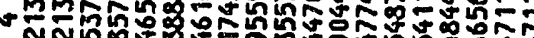

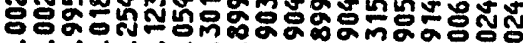
inonininobiobidonin

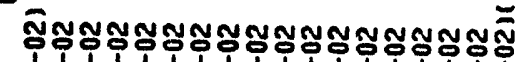

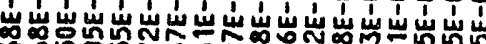

m

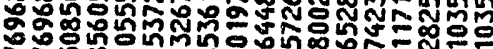

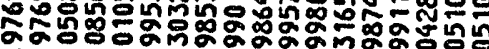
oonntiobobobobonin

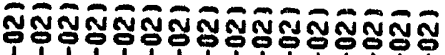

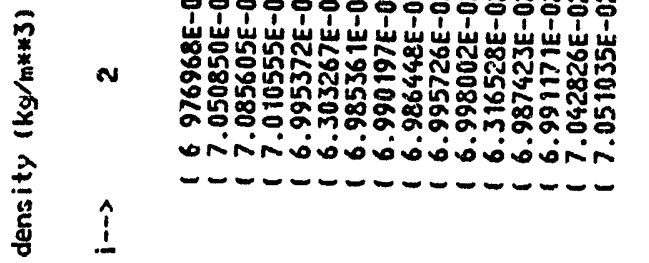

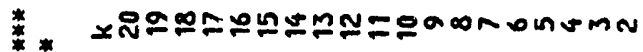

$\infty$

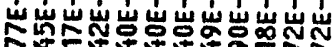
o-

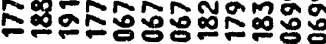

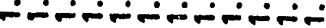
ーーーーーー ーーーー 둥후우ㅇㅠㅜ웅

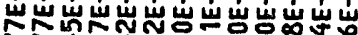
n कó

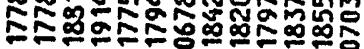
ேோோோோோன் $=$

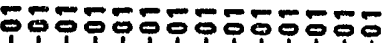
w

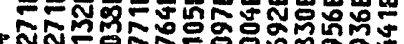

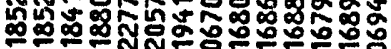

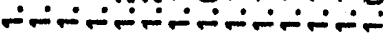

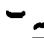

둥둥두융으

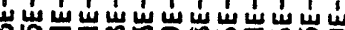

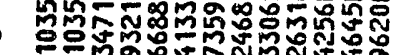

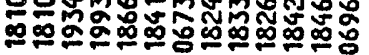

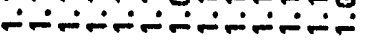

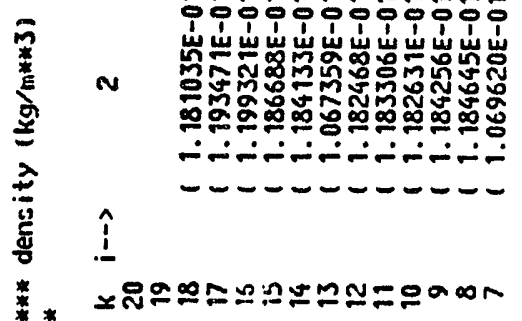

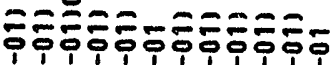




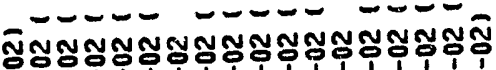

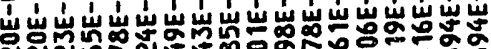

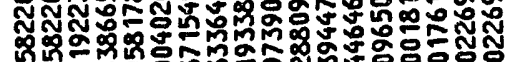

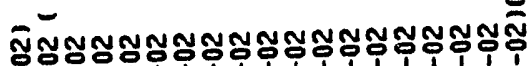

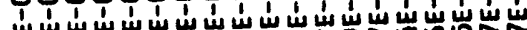

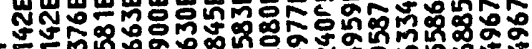

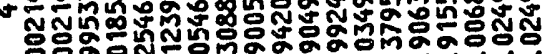

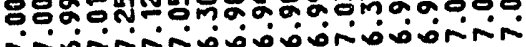

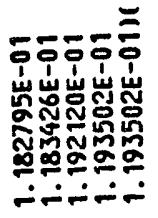

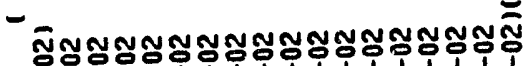

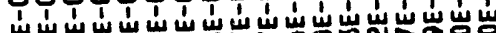

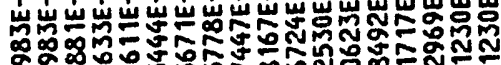

m \%

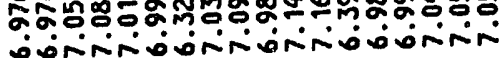

두유

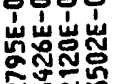

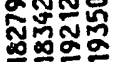

சேーே

onsma

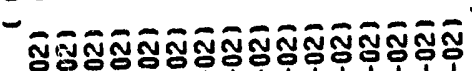

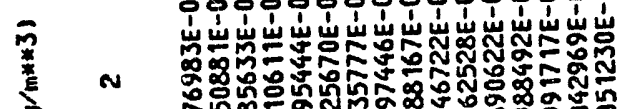

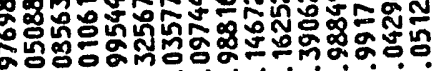

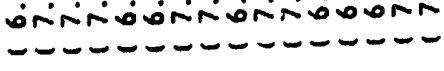

$\hat{\imath}$

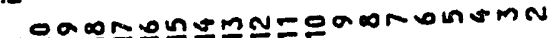

우

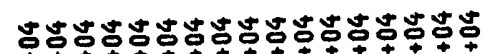

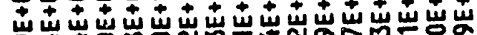

政

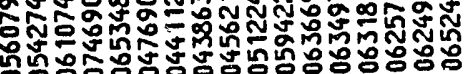

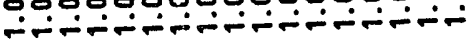

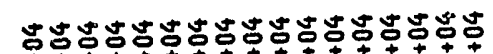

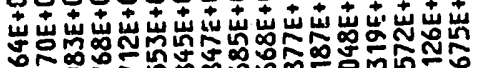

a

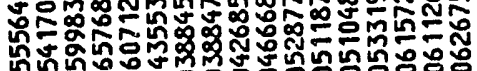

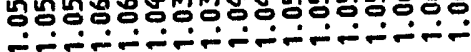

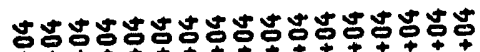

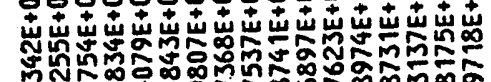

$\infty$

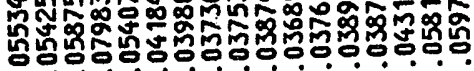

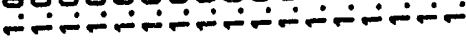

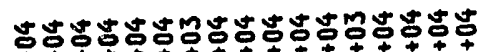

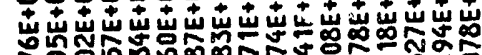

$\sim$

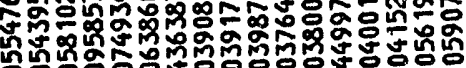

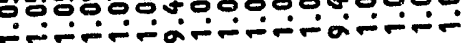

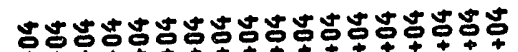

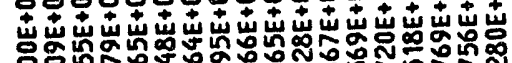

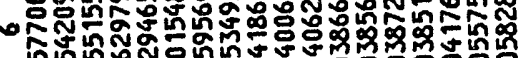

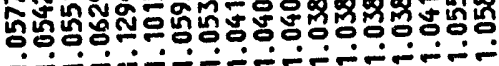

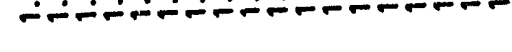

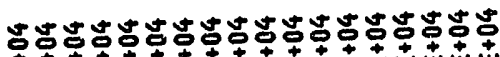

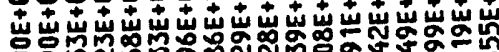

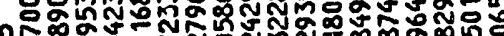

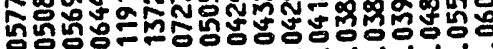

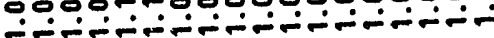

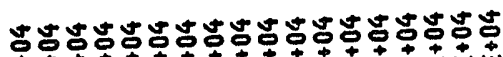

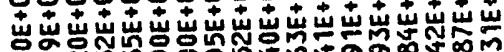

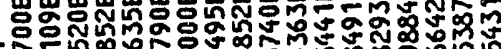

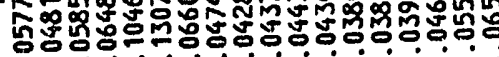

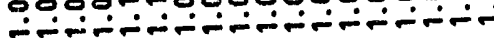

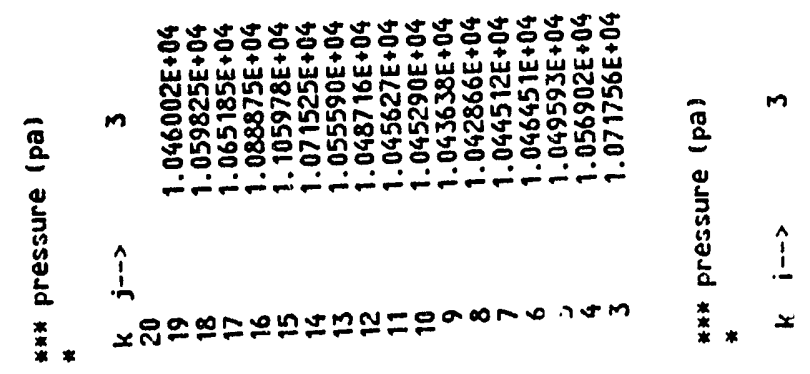




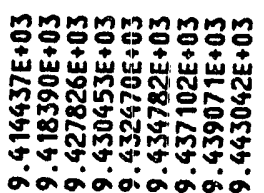

告

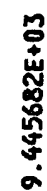

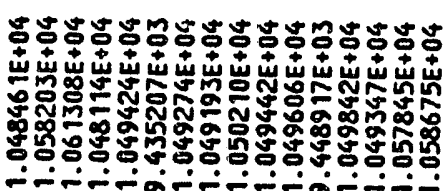

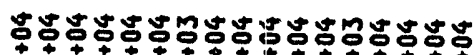

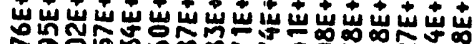

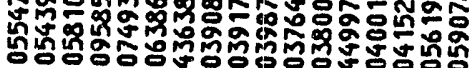

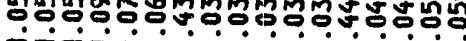

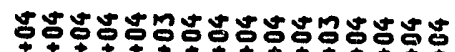

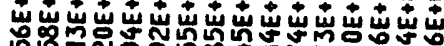

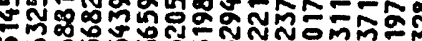

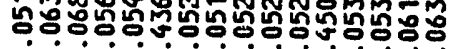

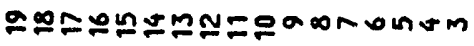

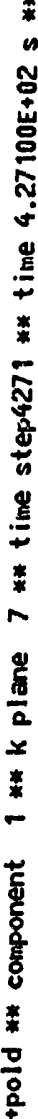

要

*
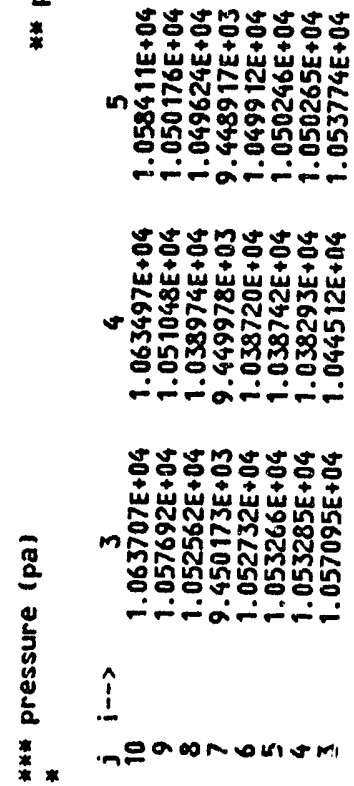

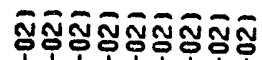
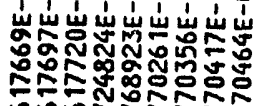

ODONTRE

minivivivis

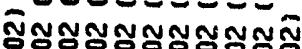

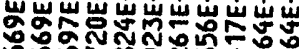

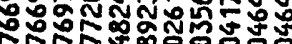

О 60 TN

minimivisusq

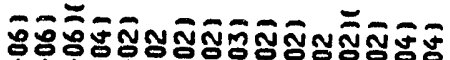

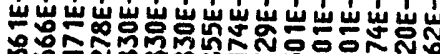

$\infty$

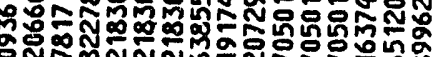

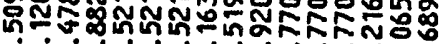

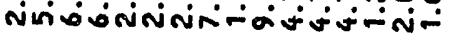

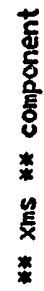

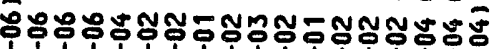

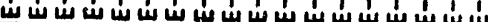

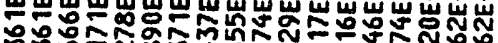

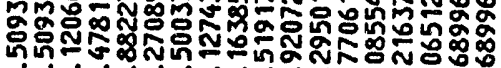

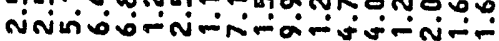

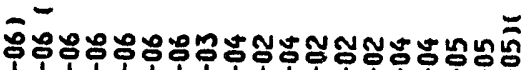

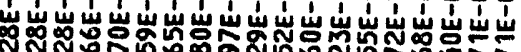

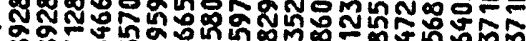

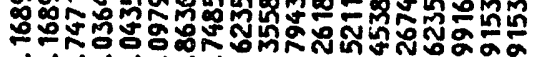

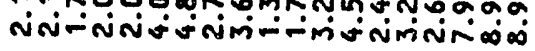

再

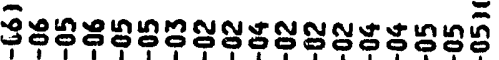

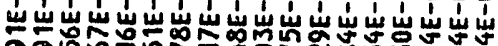

政

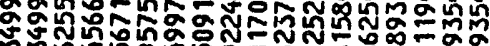

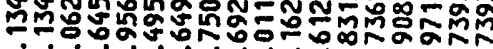

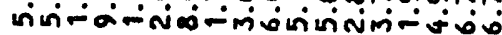

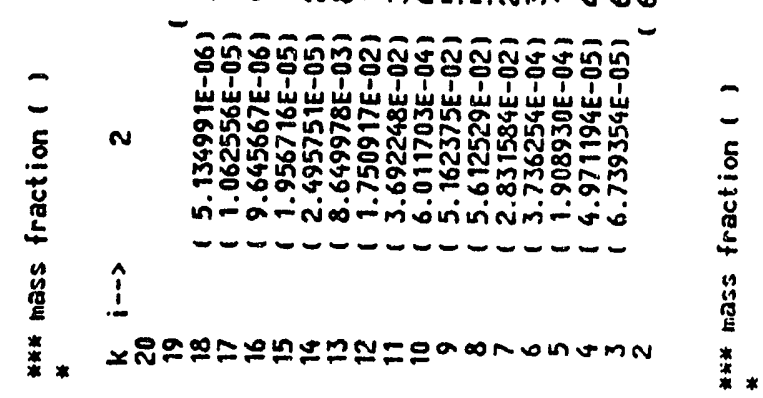




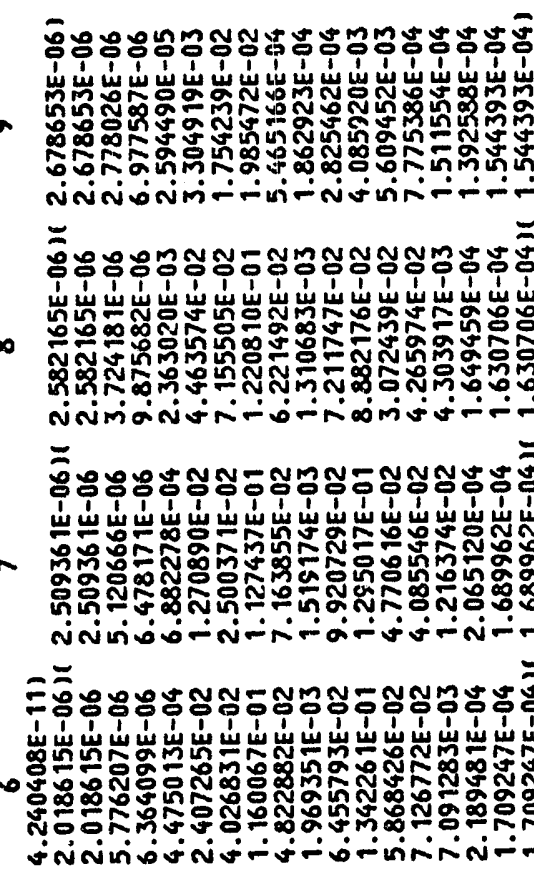

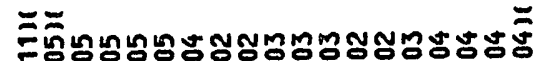

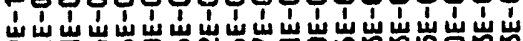

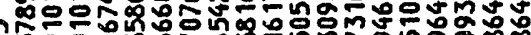

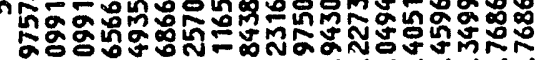
m-

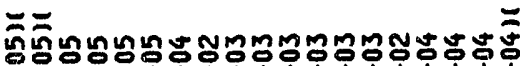

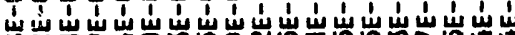

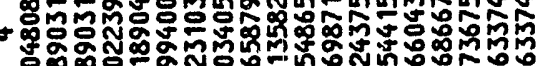

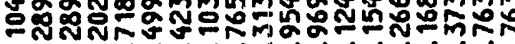

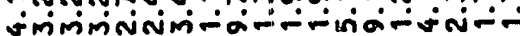

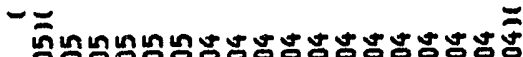

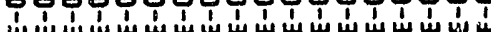

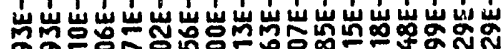

m

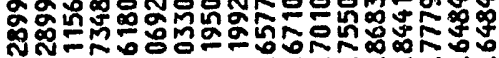
miminivini-r-

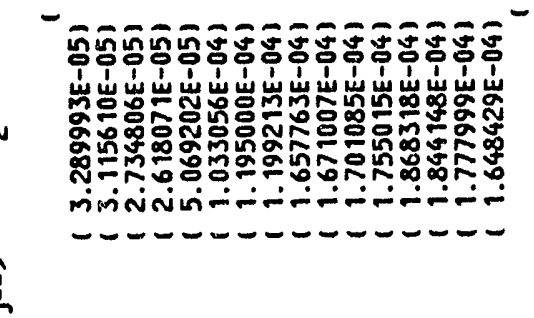

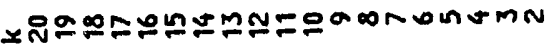

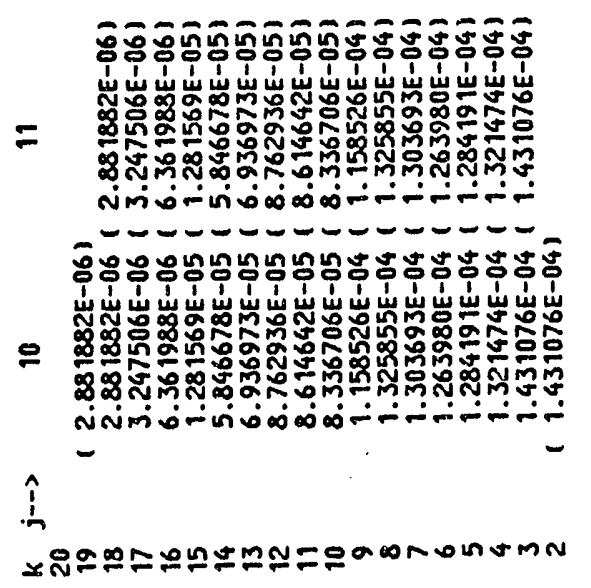

(

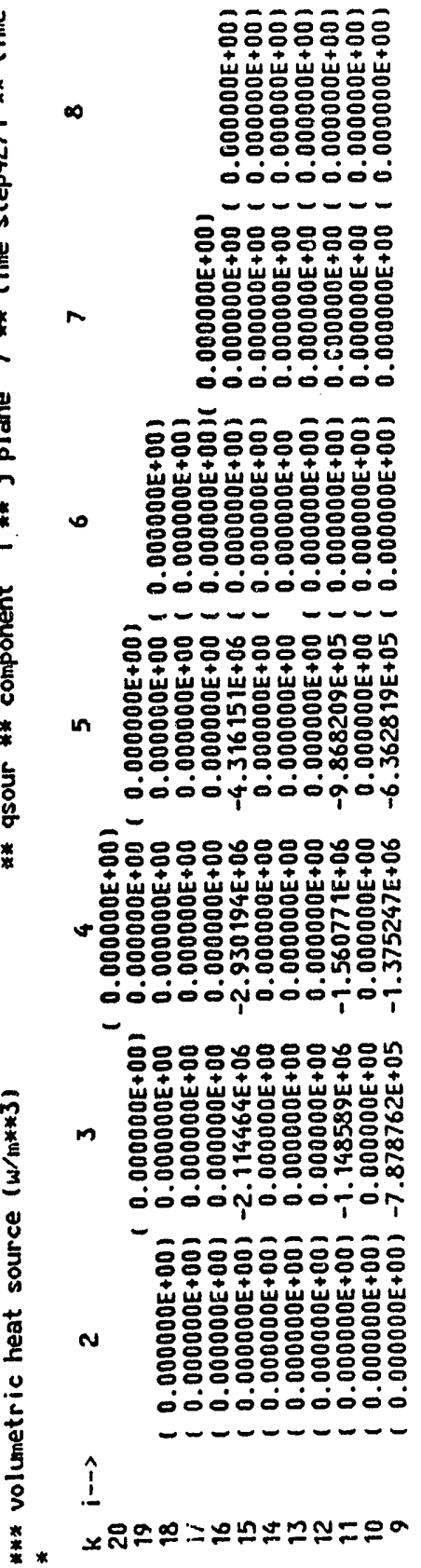




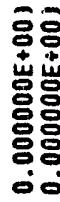

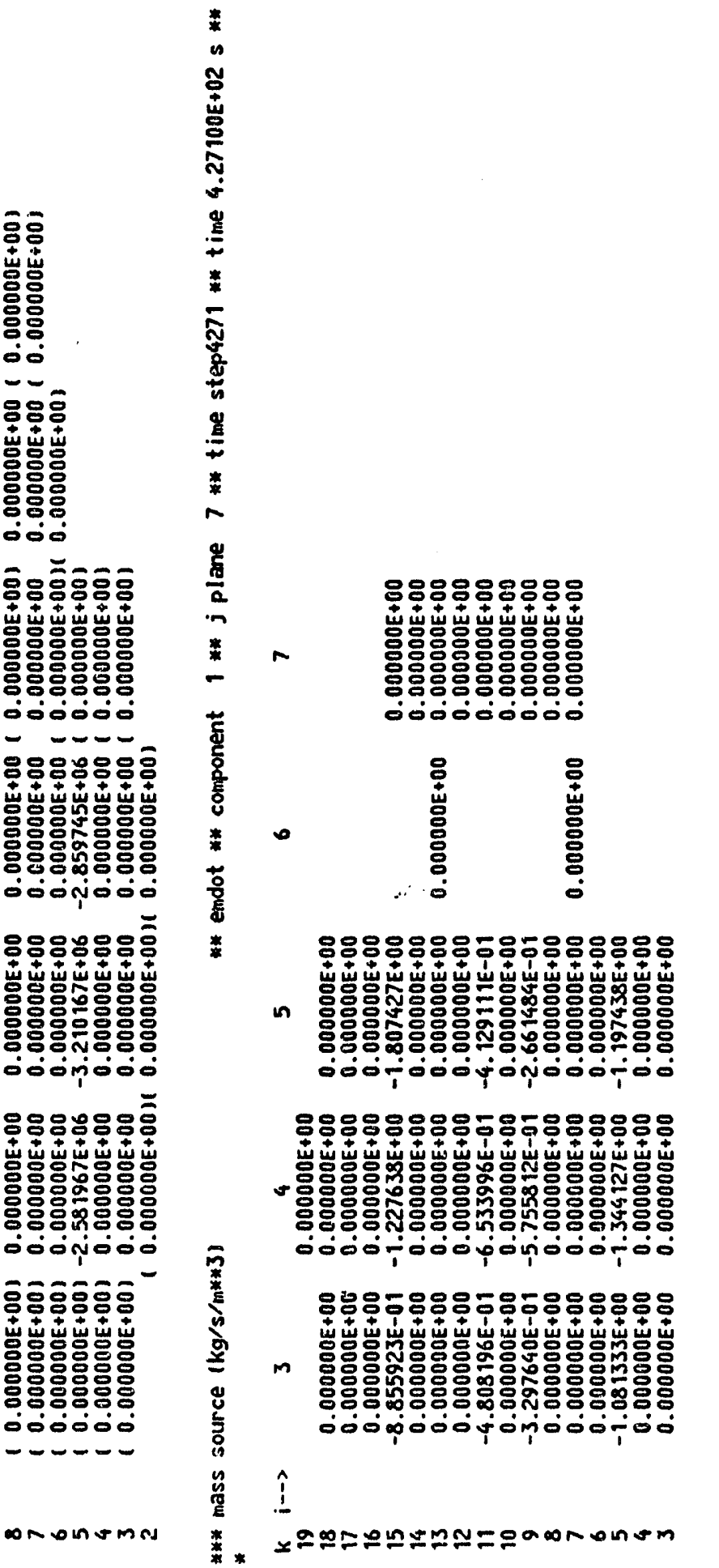

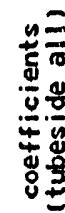

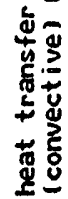

率它

竞

รั้

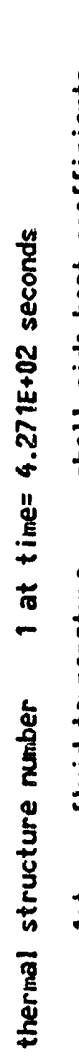
$+\frac{1}{2}$

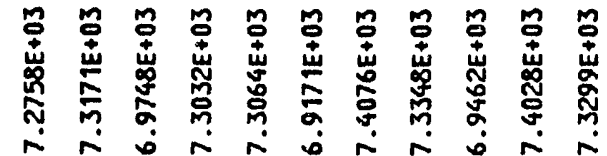

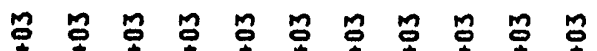

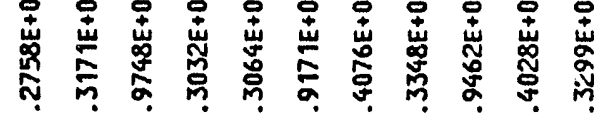
$\sim \dot{\sim} \dot{\sim} \dot{\sim} \dot{\sim} \dot{\sim} \dot{\sim}$

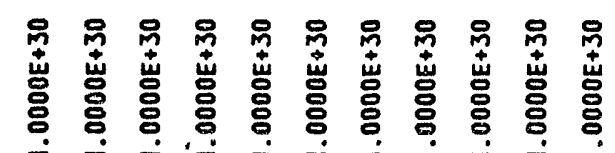

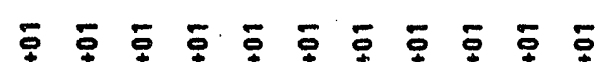

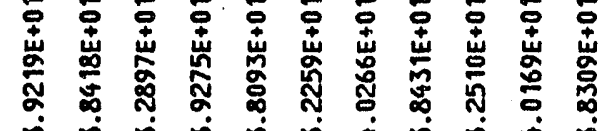
$\dot{m} \dot{m} \dot{m} \dot{m} \dot{m} \dot{m} \dot{j} \dot{m} \dot{j} \dot{m}$

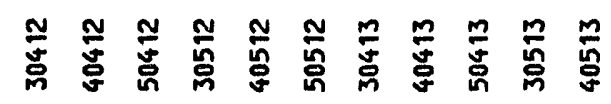

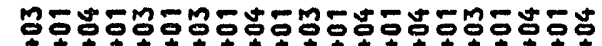

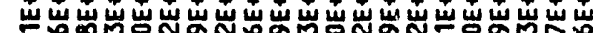
กิด

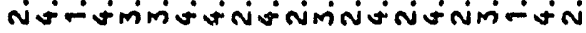

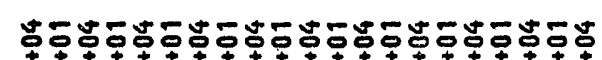

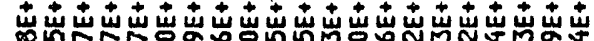

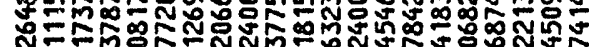

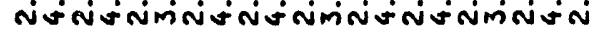

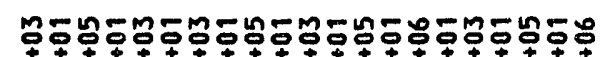

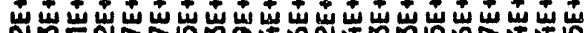
为 ตำ

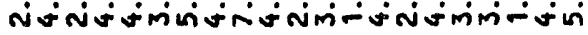

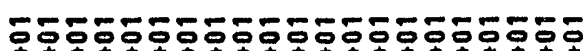

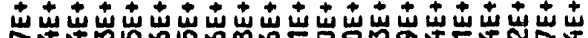

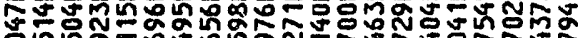

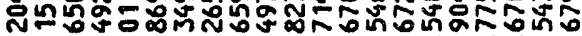

พ

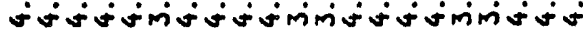

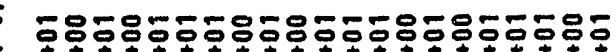

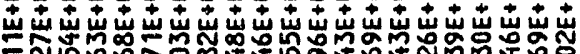

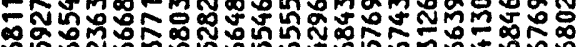

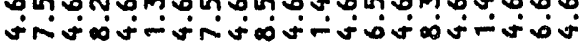

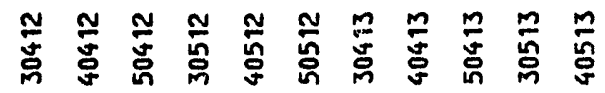

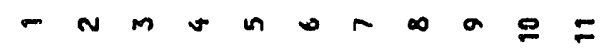




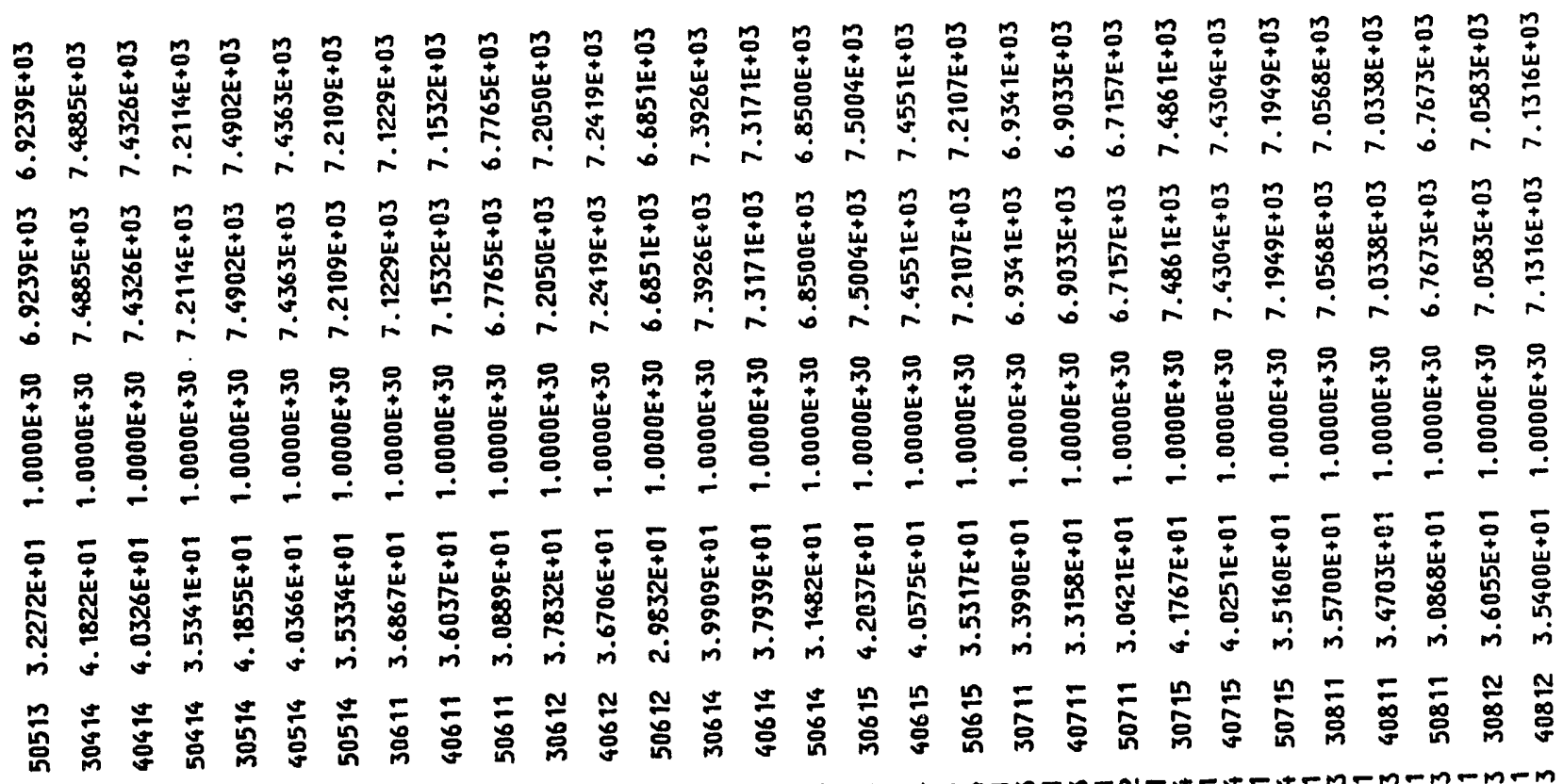

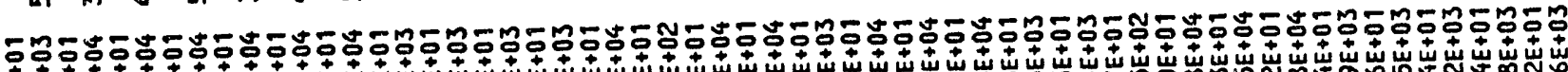

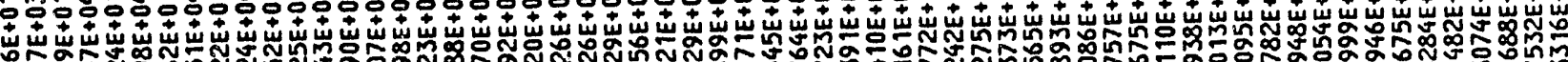

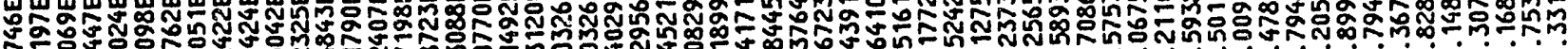
mo -

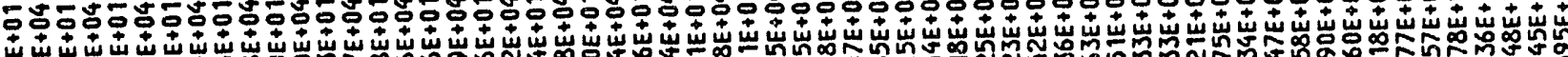

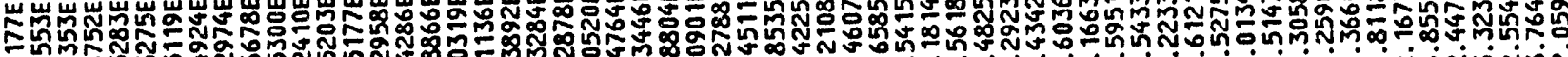

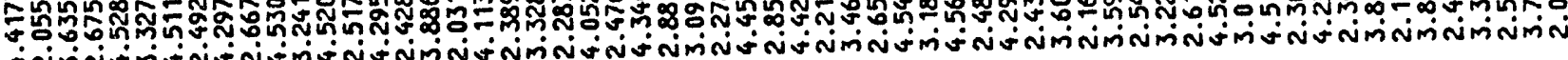

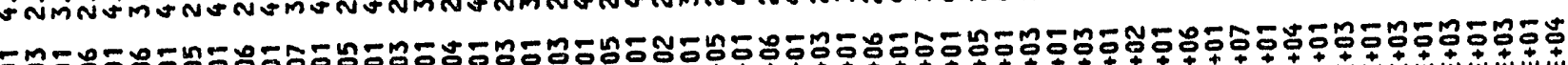

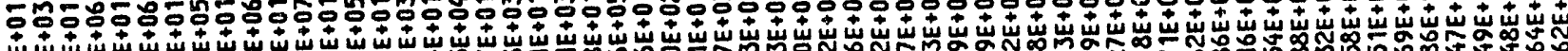

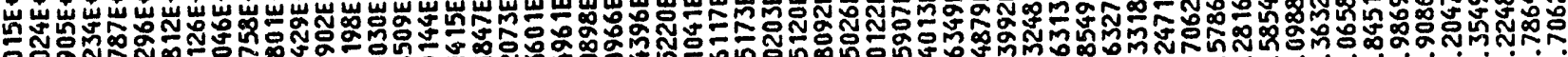

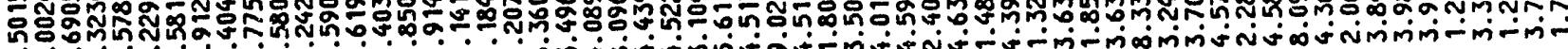

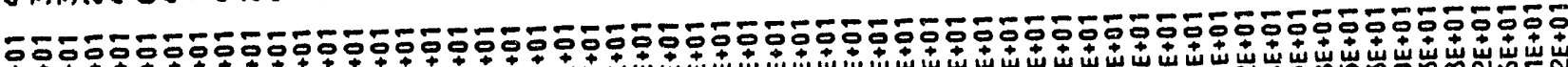

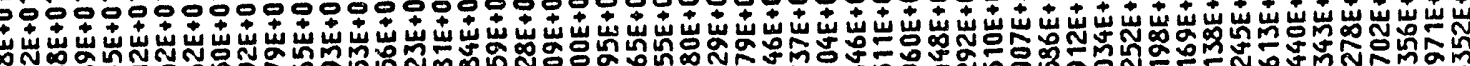

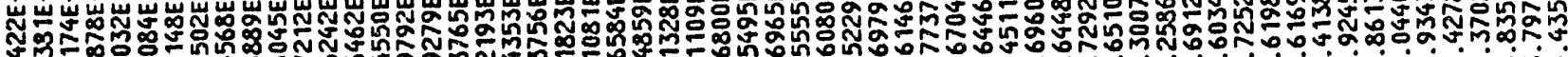

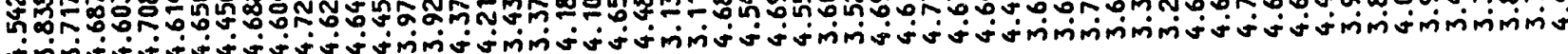

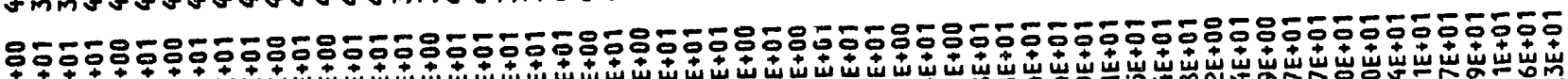

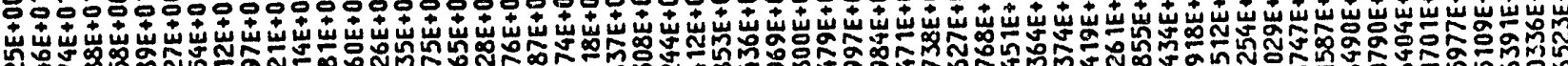

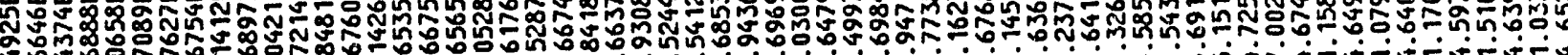
Jong

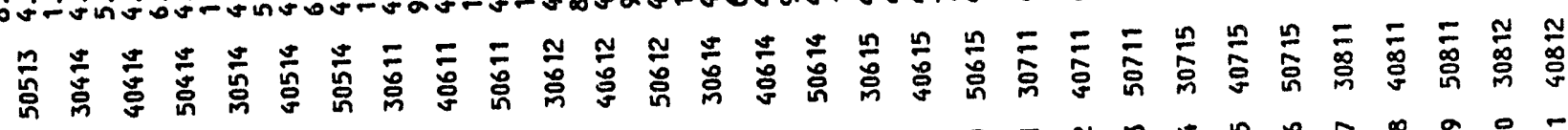

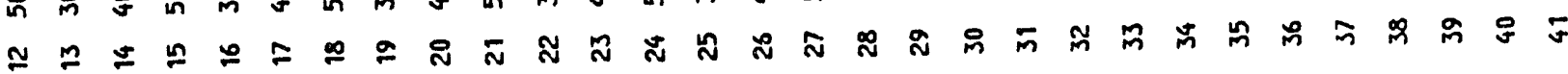




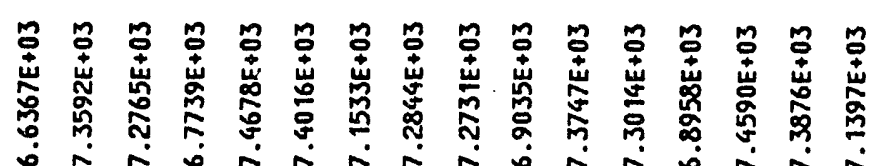

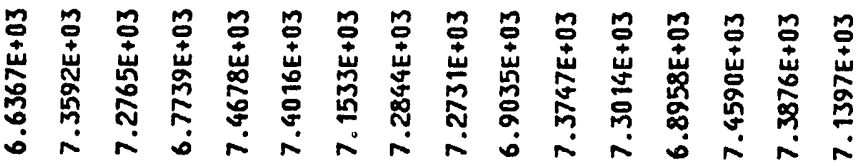

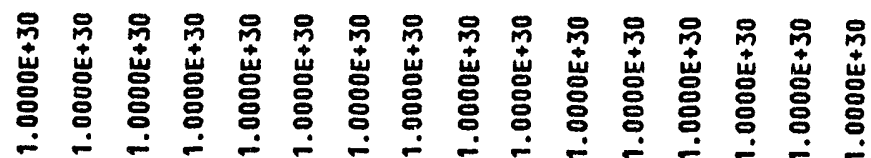

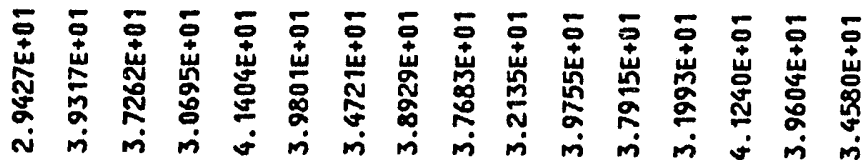

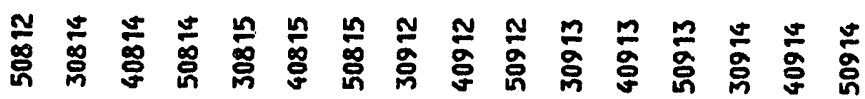

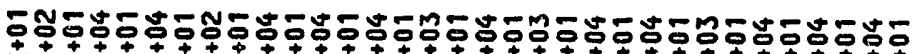

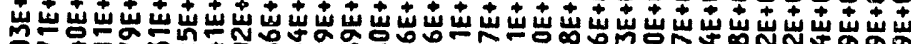
ón

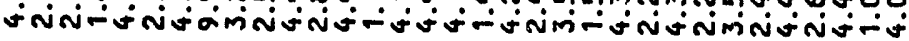

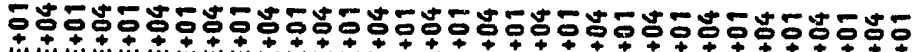

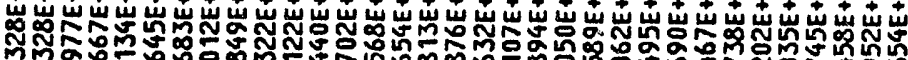

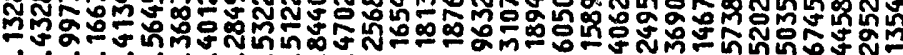

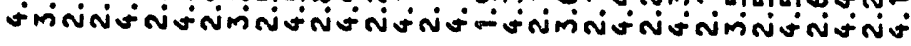

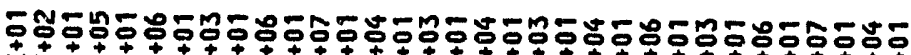

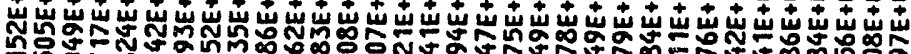

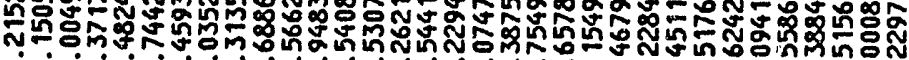

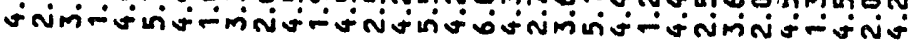

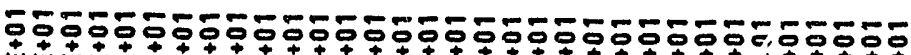

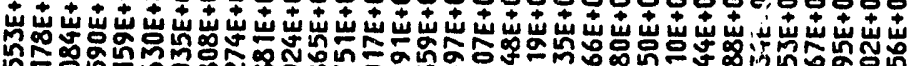

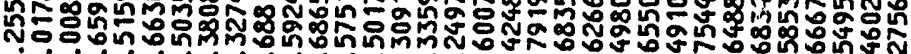

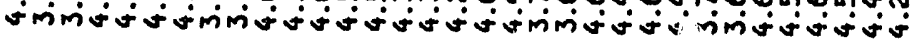

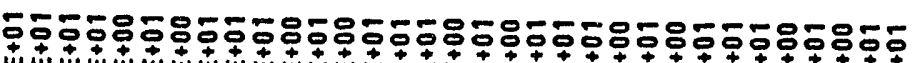

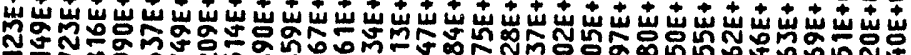

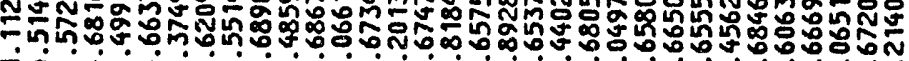

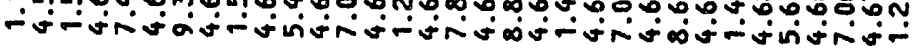

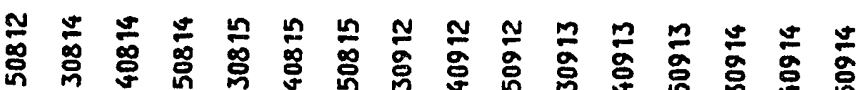

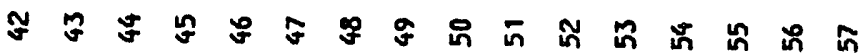

$\stackrel{n}{\mathbf{c}} \overline{\text { ॠ }}$

造要

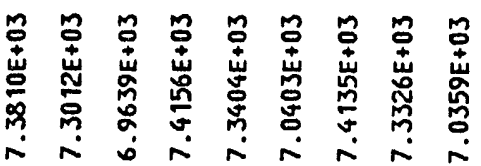

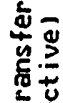

进总

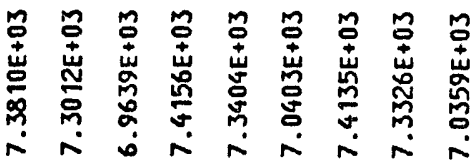

象.

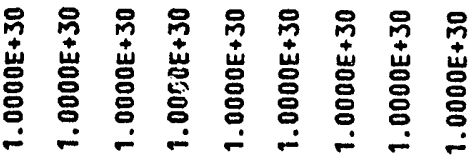

竞苔

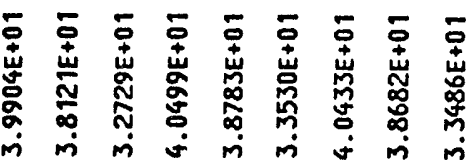

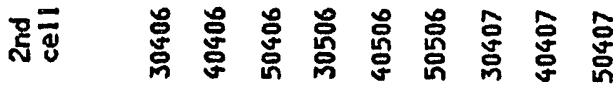

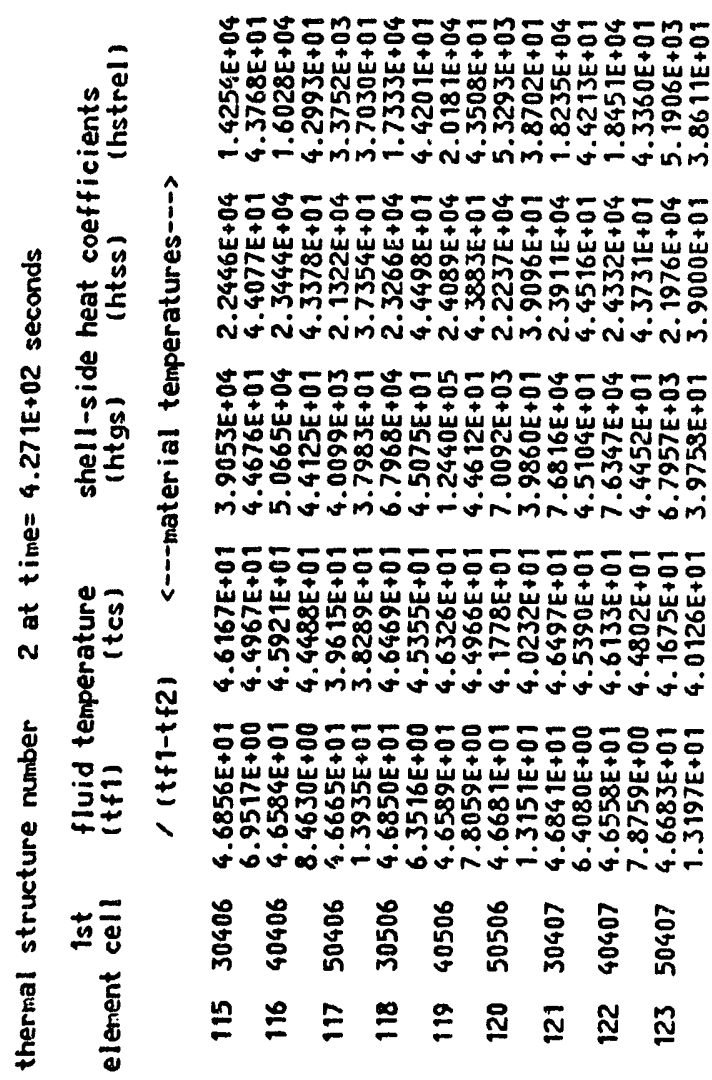




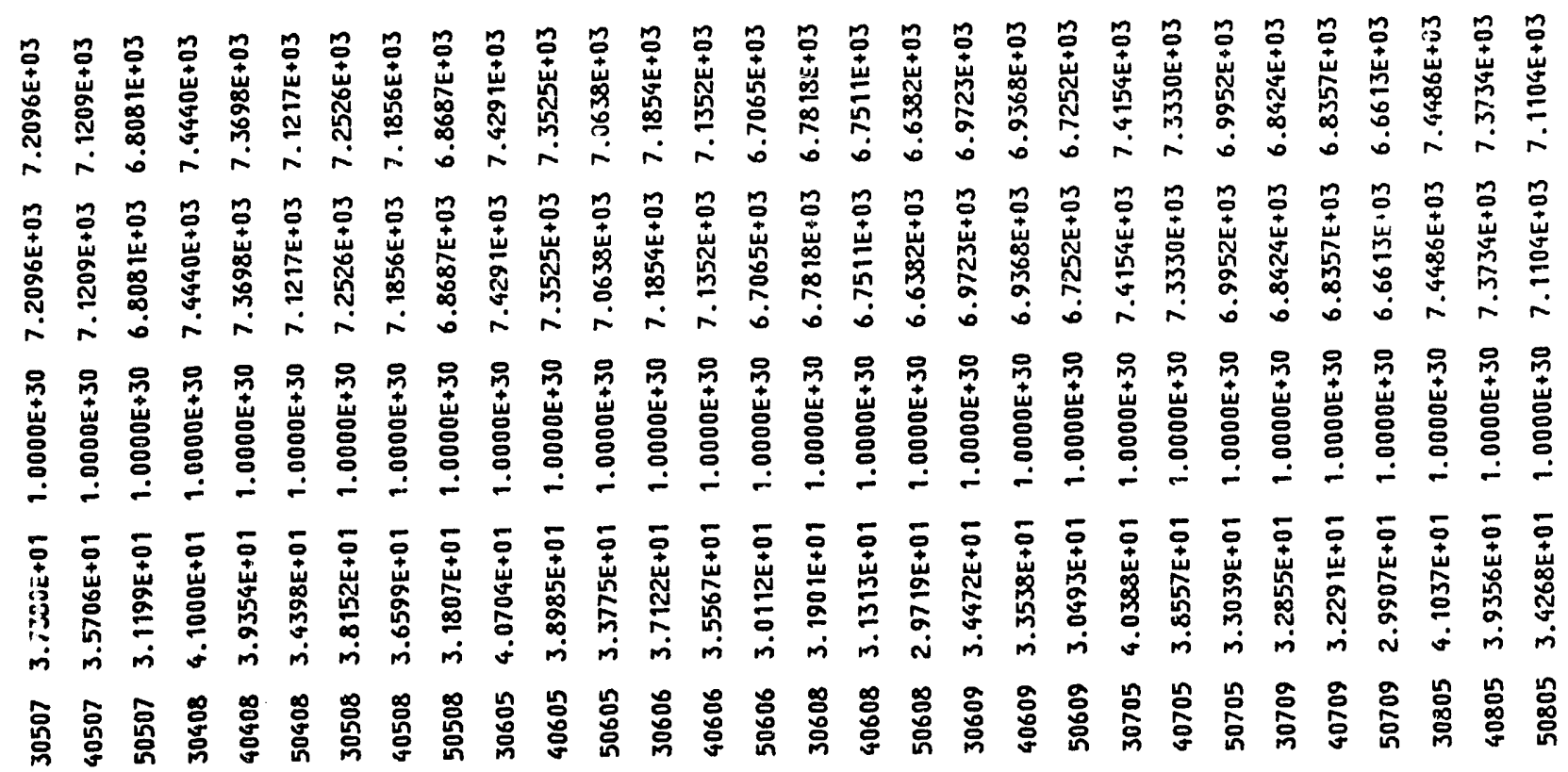

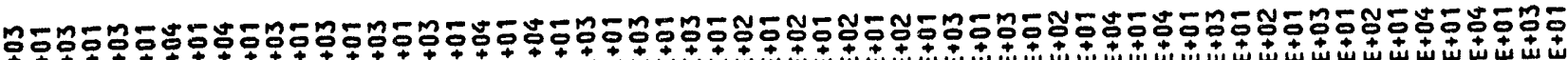

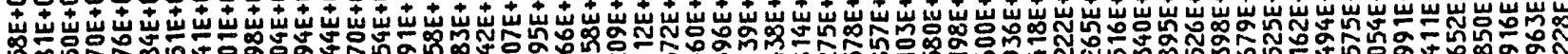

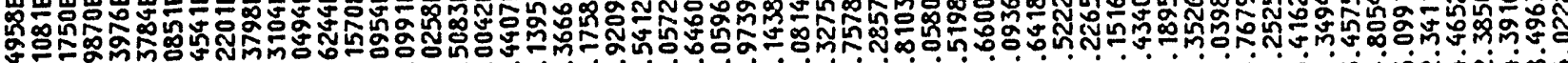

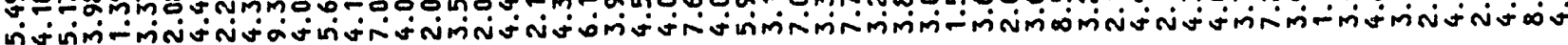

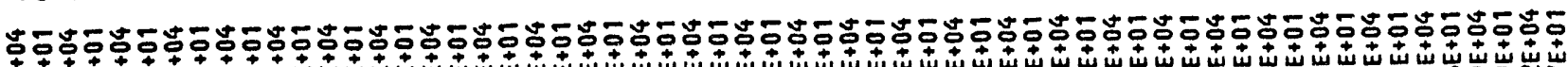

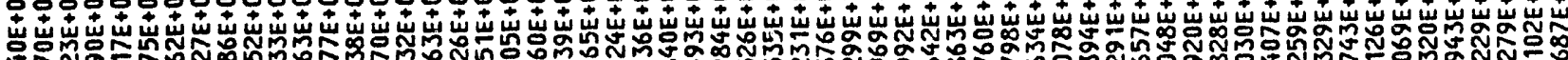

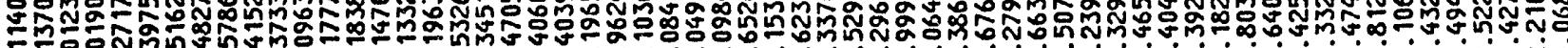
iventumát

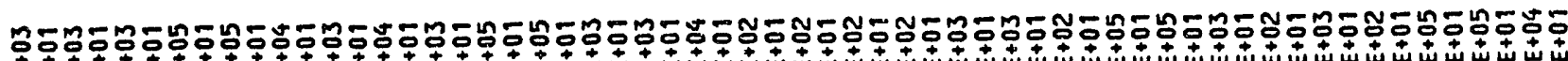

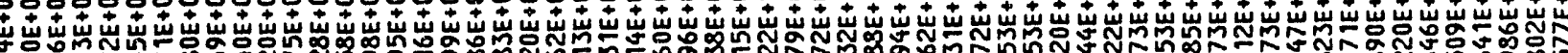

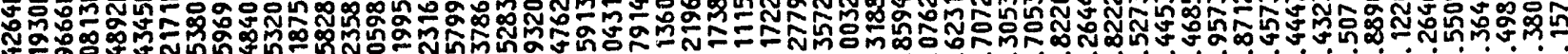

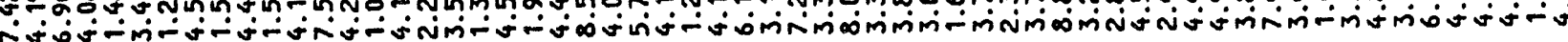

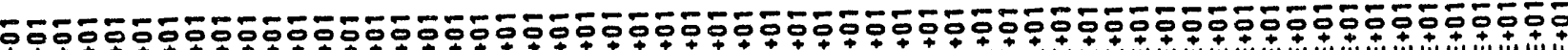

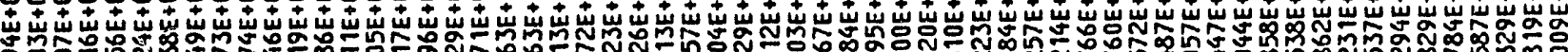

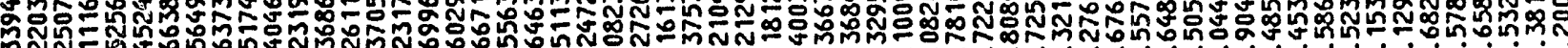

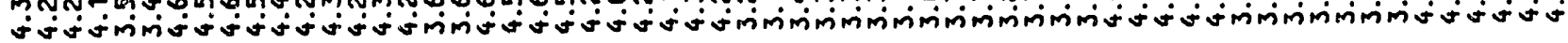

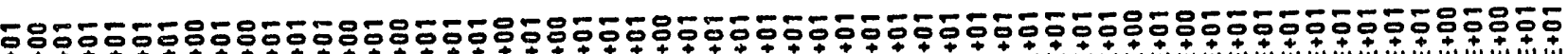

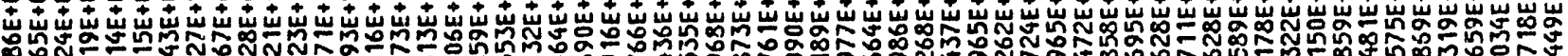


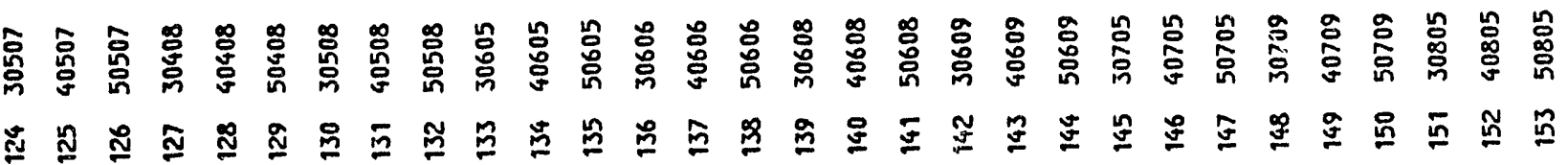




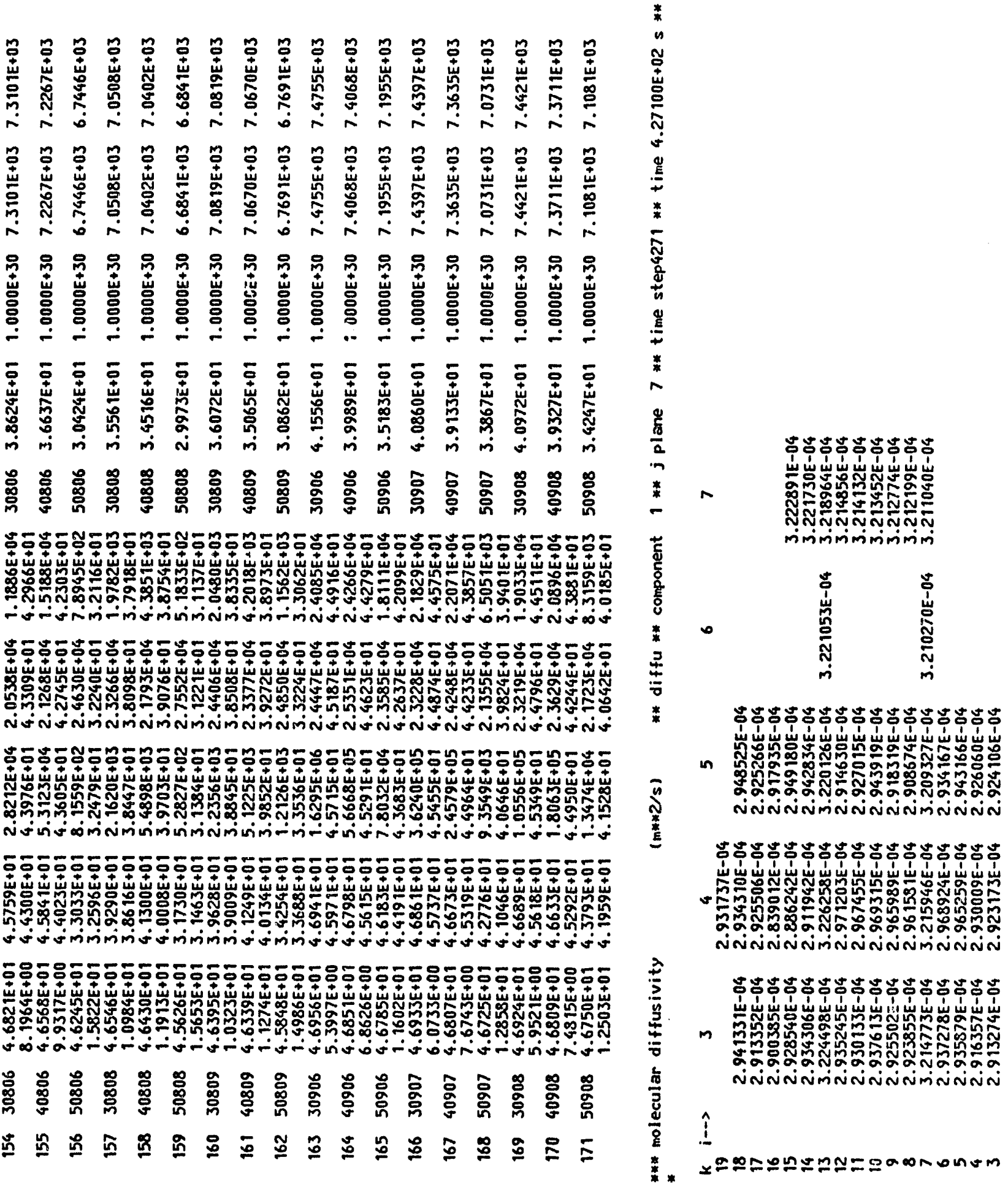




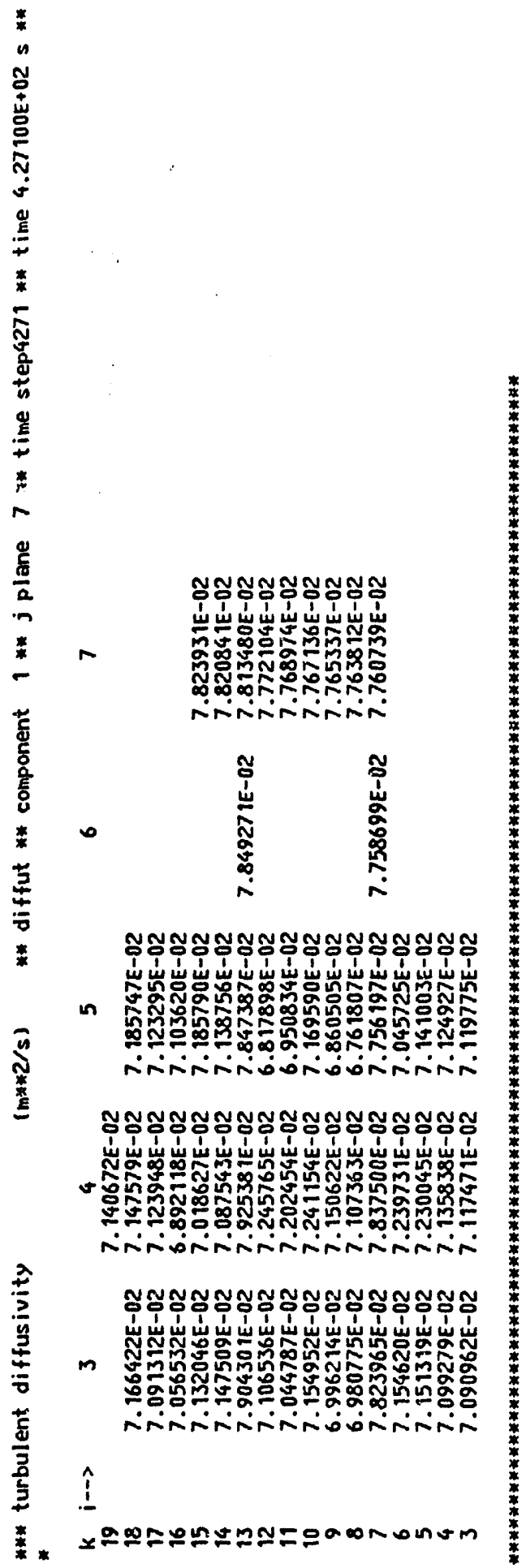




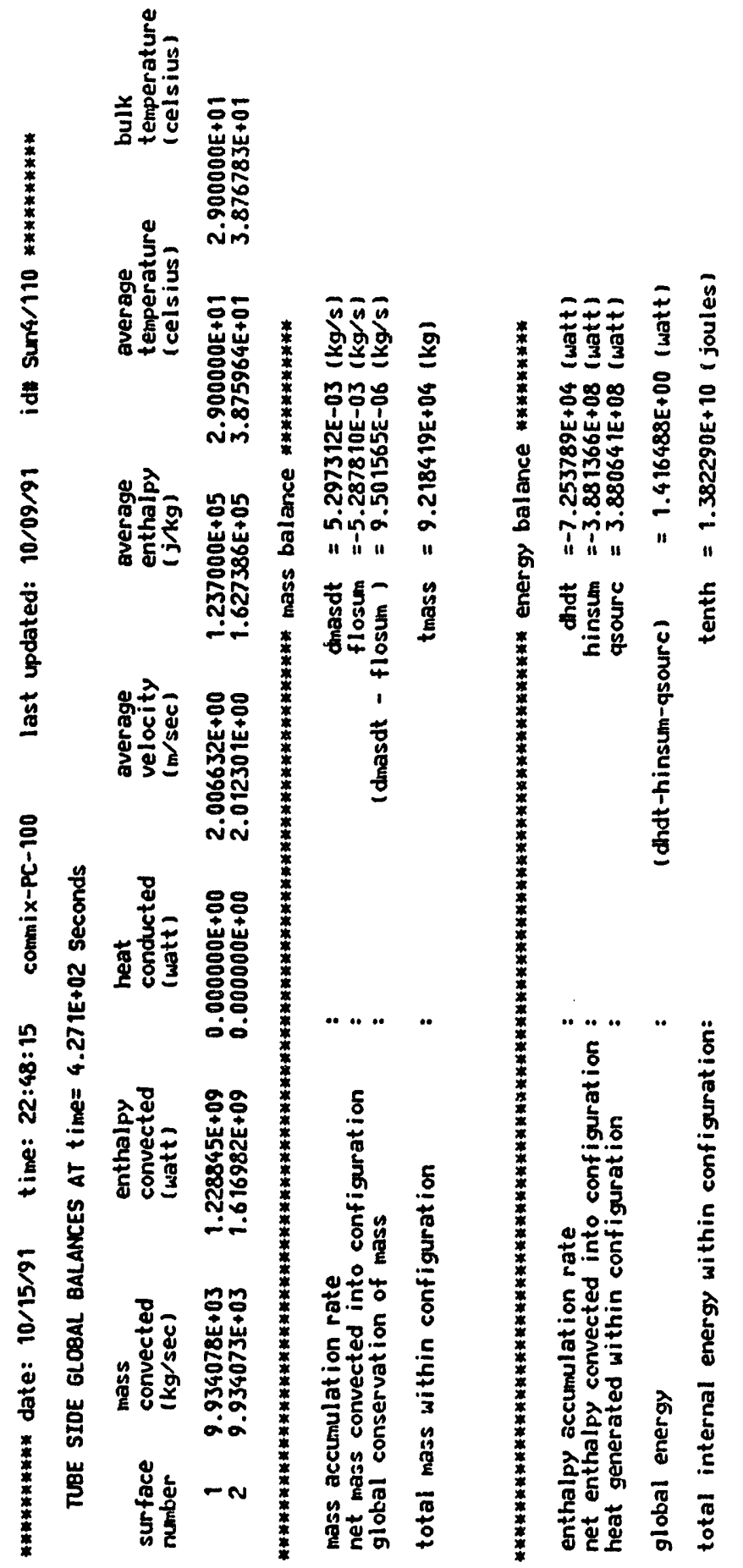

$\infty$

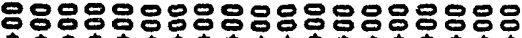

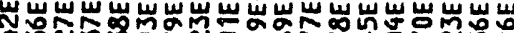

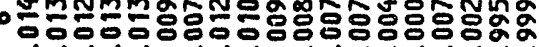

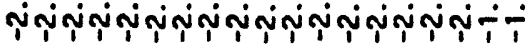

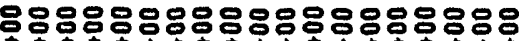

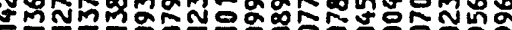
응ㅇㅇㅇㅇㅇㅇㅇㅇㅇㅇㅇㅇㅇㅇㅇㅇ영

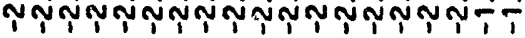

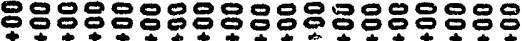

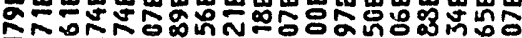

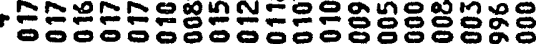

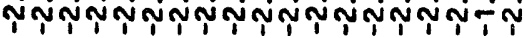

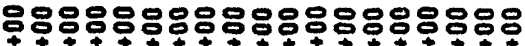

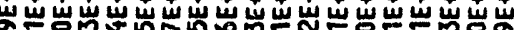

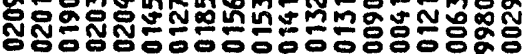
NTNNTNNNTNNNTNTNNTYTT 용요용요용ㅇㅇㅇㅇㅇ용ㅇㅇ용ㅇㅇㅇ

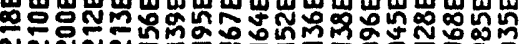

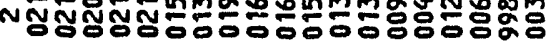

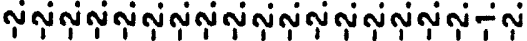

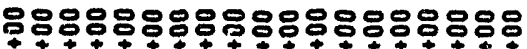

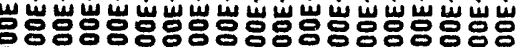
- 양양융ㅎㅇㅇㅇㅇㅇㅇㅇㅇㅇㅇㅇㅇㅇㅇㅇㅇㅇㅇㅇㅇㅇㅇㅇㅇㅇㅇㅇㅇㅇㅇㅇㅇ Dó0000000000000

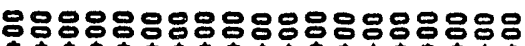

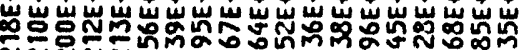

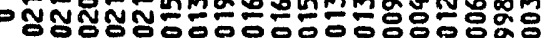

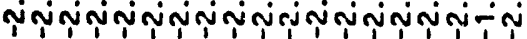

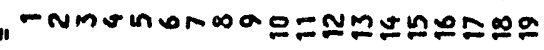




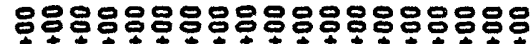

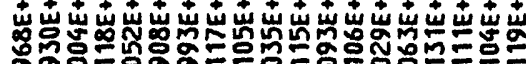

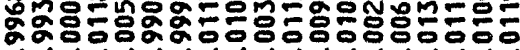

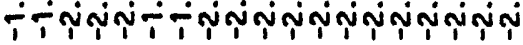

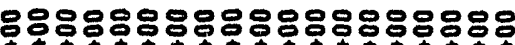

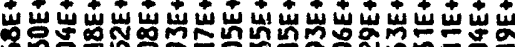

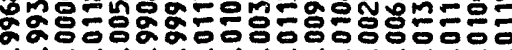
- -NNNTTNNNNNNNNNNNN

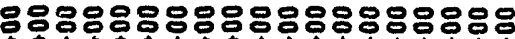

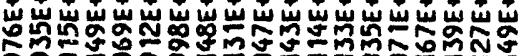

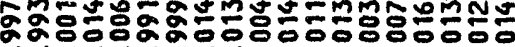

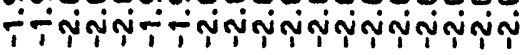

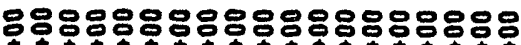

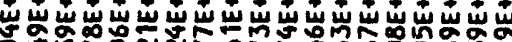

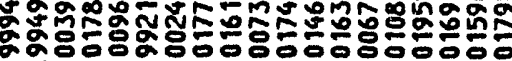

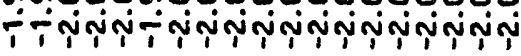

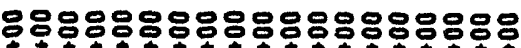

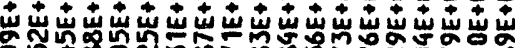

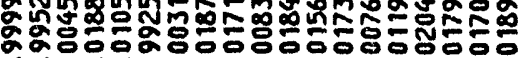

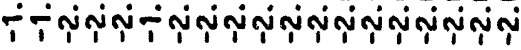

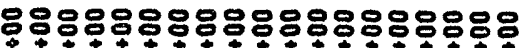

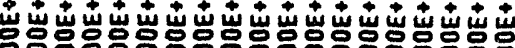

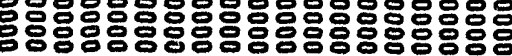

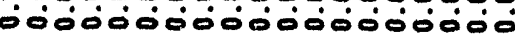

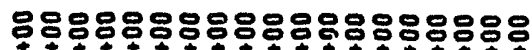

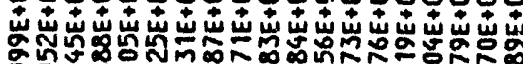

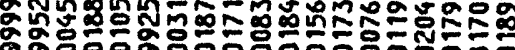
- -

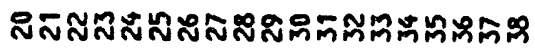

$\infty$

N

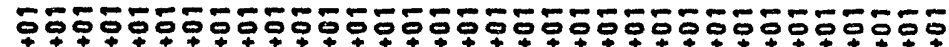

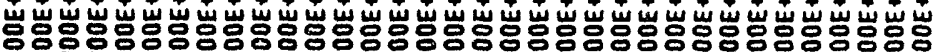

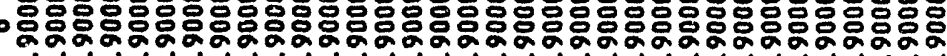

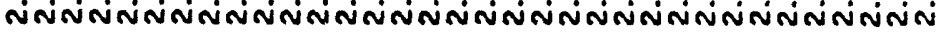

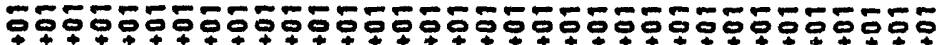

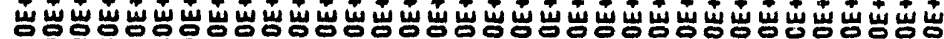

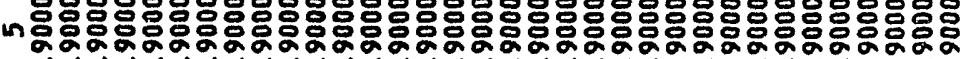

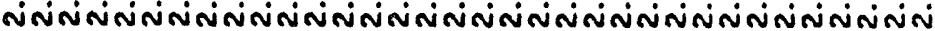

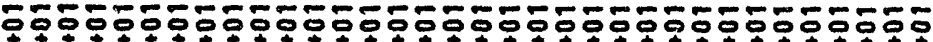

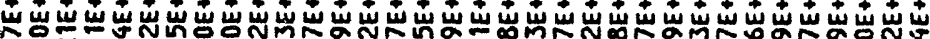

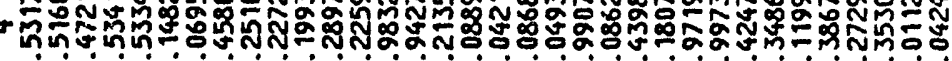

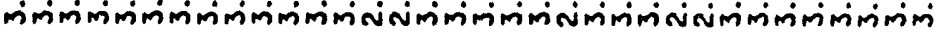

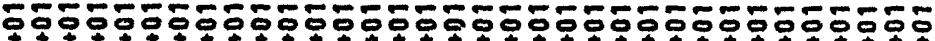

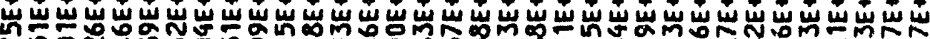

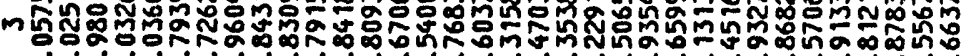

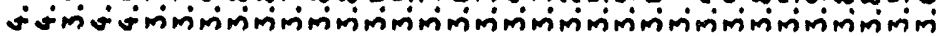

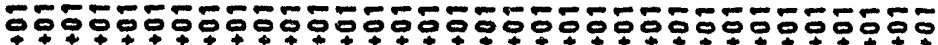

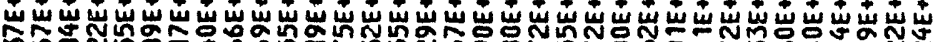

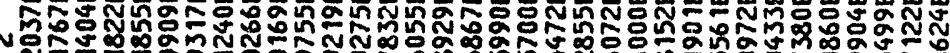

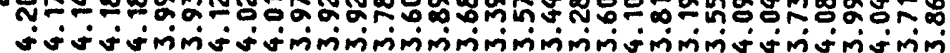

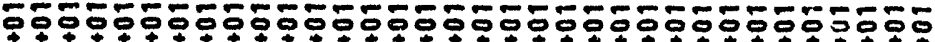

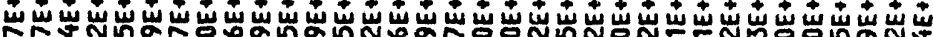

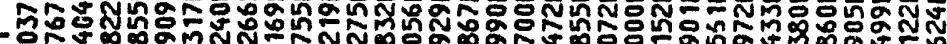
On

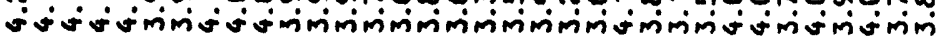

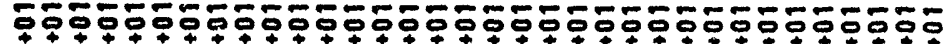

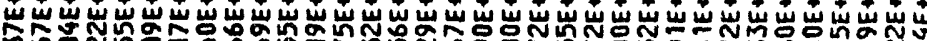

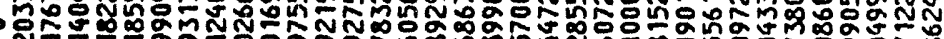

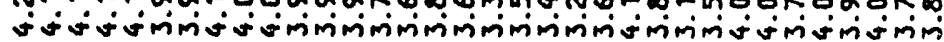

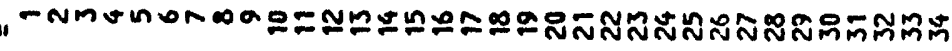




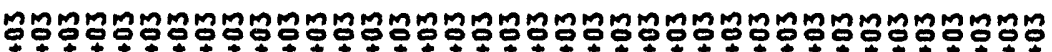

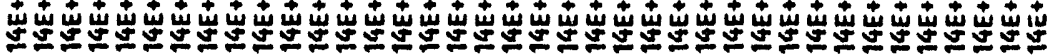
๑ -

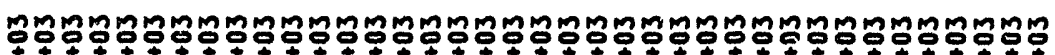

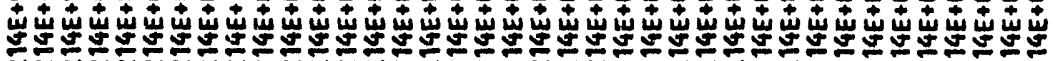
n -

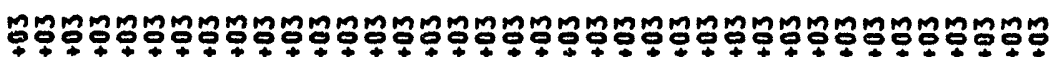

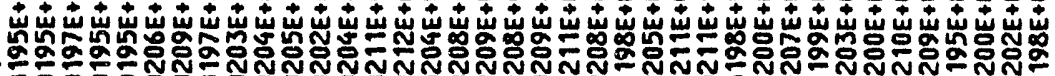

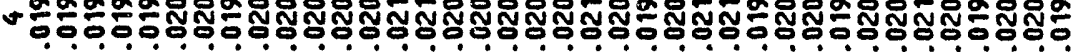

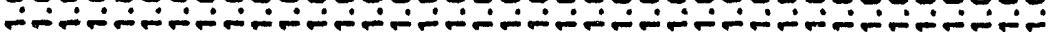

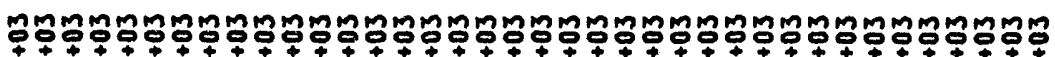

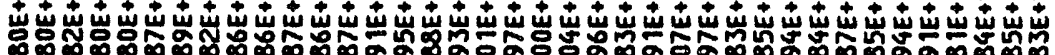
n

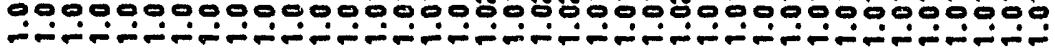

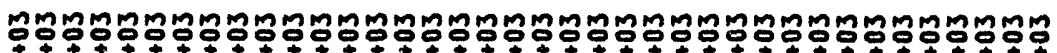

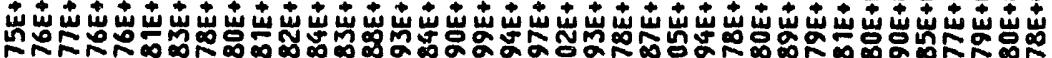

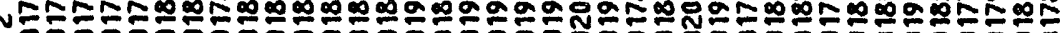

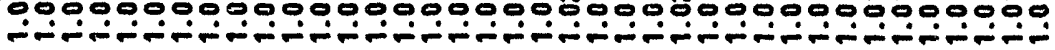

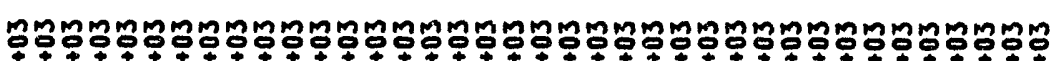

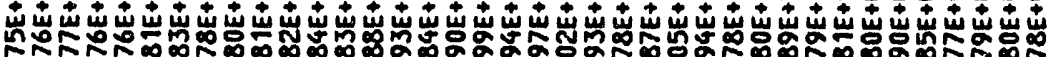

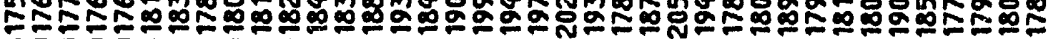

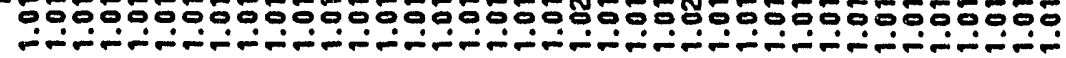

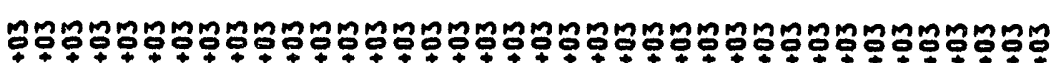

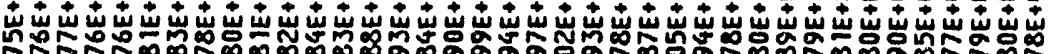

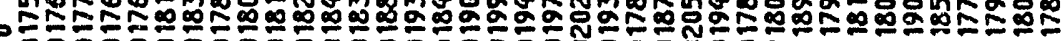
00000000000 "

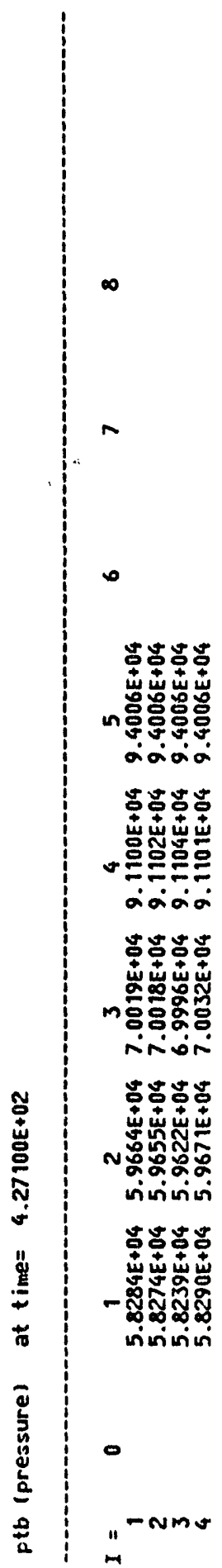




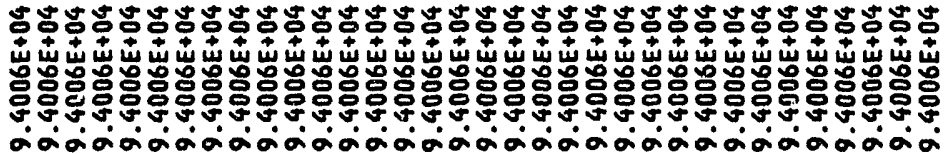

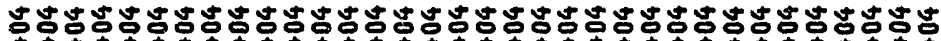

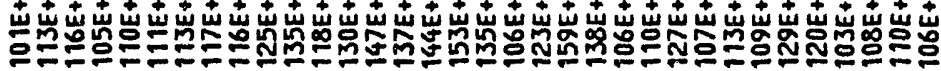

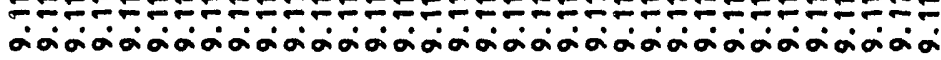

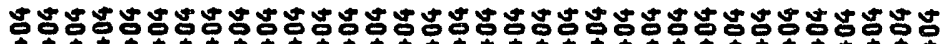

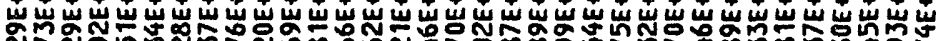

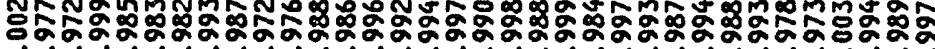

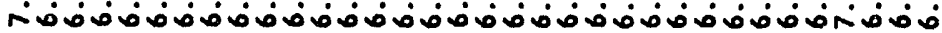

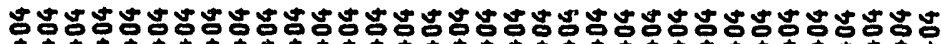

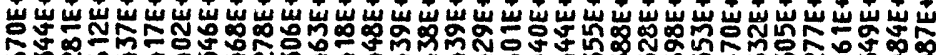

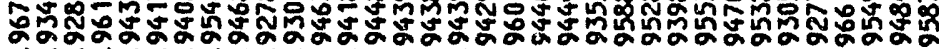

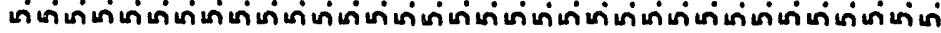

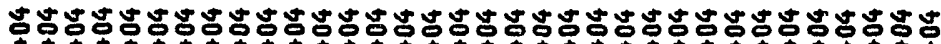

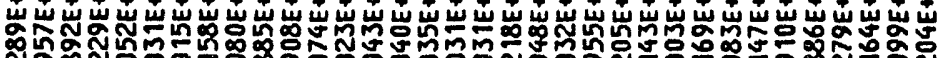
స్م

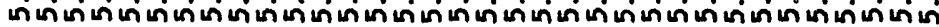

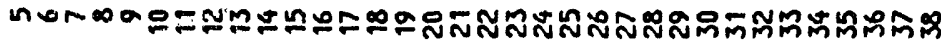

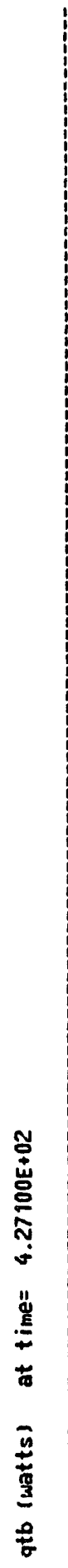

$\infty$

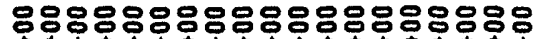

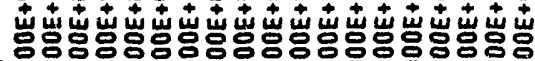
n

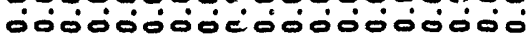

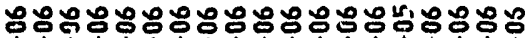

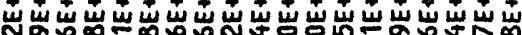

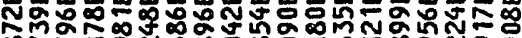

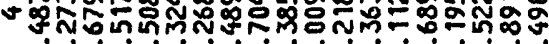

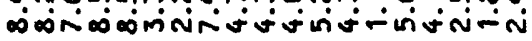

๕ะะะัะะษะะะะะะะะะะะ

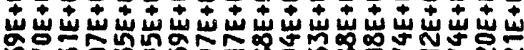

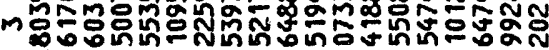

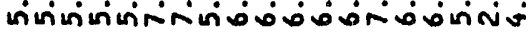

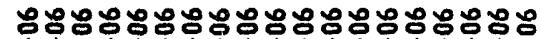

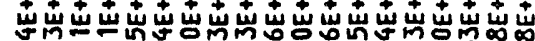

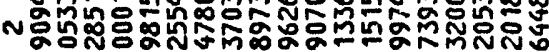
miviminivis vinimi-miñN



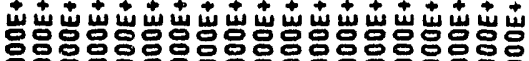

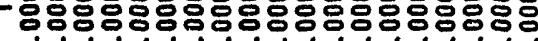
-000000000000000000

○

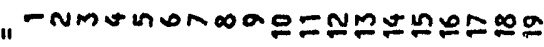




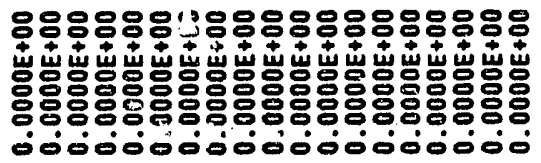

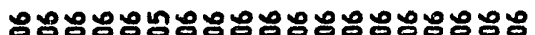

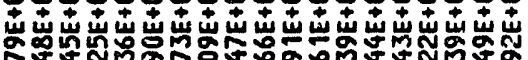

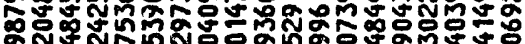

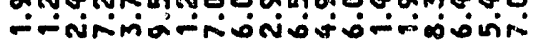

๑ะษะะ

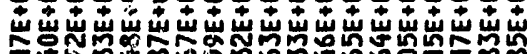

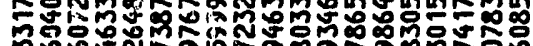

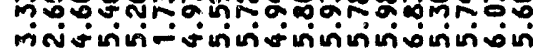

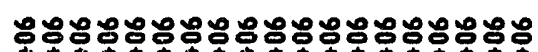

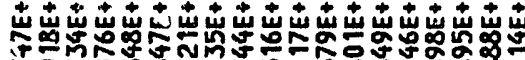

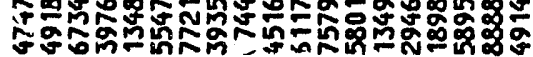
N

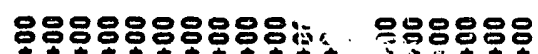

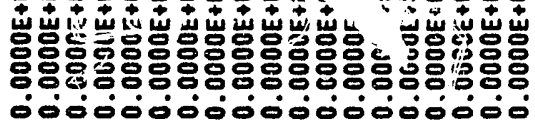




\section{Appendix E. Sensitivity Study: Effect of Condenser Inlet Air Mass Fraction and Exit Mass Flow Rate of Air Extraction System on Steam Condensation Rate}

As a steam/air mixture passes a bank of condensing tubes in a power plant condenser, noncondensible air tends to accumulate at the interface between metal surfaces and condensate films. Because air is a poor conductor, its presence on a condenser tube surface could significantly retard the overall steam condensing rate. To improve condenser performance, an air extraction system or ejector is used to remove noncondensible air. The amount of air to be removed depends on the air mass concentration and its distribution in the shell side and the extraction flow rate through the ejectors. Thus, a sensitivity study was performed to study the effects of inlet air mass fraction and mass flow at the exit (ejector) on the steam condensing rate. The following cases were investigated in this sensitivity study.

1. Varying inlet air mass fraction: All the conditions are identical to the sample problem in Appendix $D$ except that the inlet mass fraction is changed from $4.11 \times 10^{-5}$ in the Sample Problem to $0.2 .05 \times 10^{-5}, 8.21 \times 10^{-5}$, and $16.42 \times 10^{-5}$.

2. Varying the exit mass flow rate: All the conditions are identical to Case 1 except that the exit mass flow rate is increased by 100 percent.

Table E-1 lists the conditions imposed in each case and the corresponding calculated steam condensation rates. These results are also shown in Fig. E.1, where it is evident that decreasing the inlet air mass fraction or increasing the exit mass flow rate increases the steam condensation rate in the condenser. Figs. E.2-E.4 show the constant air mass fraction line for cases $1.3,1.4$, and 2.4 (defined in table E-1), respectively.

Note that whereas Figs. E.2 and E.3 show that the more air mass enters the condenser. the more air will accumulate near the tube bank, resulting in decreasing the steam condensation rate, Figs. E.3 and E.4 show that increasing the exit mass flow rate results in reducing the air accumulating near the tube bank and increasing the steam condensation rate. 
Table 1E-1. Effect of condenser inlet air mass fraction and exit mass flow rate of an air extraction system on steam condensation rate

\begin{tabular}{cccc}
\hline Inlet Air Mass & $\begin{array}{c}\text { Mass Flow Rate } \\
\text { Fraction } \\
\left(10^{-5}\right)\end{array}$ & $\begin{array}{c}\text { Extraction Pipe } \\
(\mathbf{k g} / \mathrm{s})\end{array}$ & $\begin{array}{c}\text { Calculated } \\
\text { Steam Condensation } \\
(\mathrm{kg} / \mathrm{s})\end{array}$ \\
\hline 1.1 & 0 & 0.0505 & 208.9 \\
1.2 & 2.05 & 0.0505 & 178.1 \\
$1.3 \mathrm{a}$ & 4.11 & 0.0505 & 162.5 \\
1.4 & 8.21 & 0.0505 & 138.6 \\
1.5 & 16.42 & 0.0505 & 106.7 \\
2.1 & 0 & 0.1010 & 208.9 \\
2.2 & 2.05 & 0.1010 & 188.1 \\
2.3 & 4. & 0.1010 & 176.6 \\
2.4 & 8.21 & 0.1010 & 160.0 \\
2.5 & 16.42 & 0.1010 & 137.6 \\
\hline
\end{tabular}

aSample Problem in Appendix D 


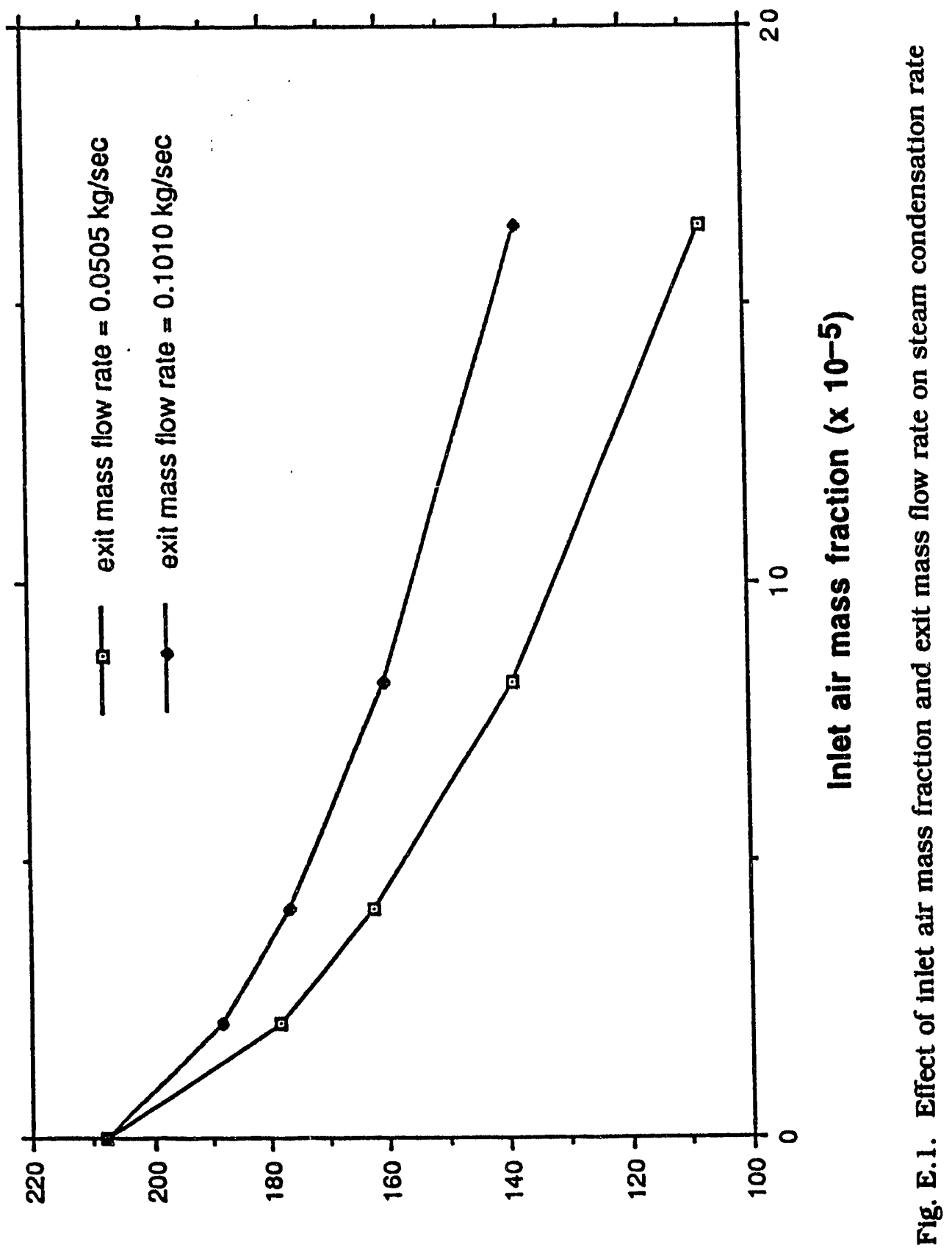

(s/Бy) әңеג uo!jesuәpuos meәts 


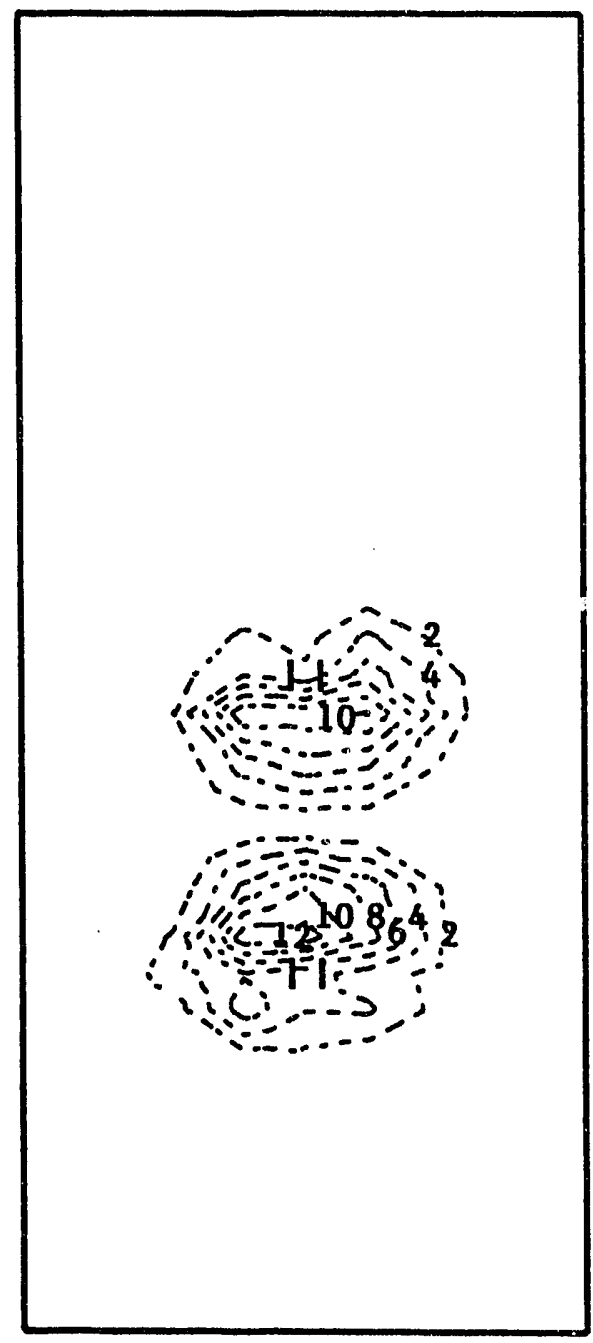

Fig. E.2. Constant air mass fraction lines (\%) at $I=5$ plane for Case 1.3

Inlet air mass fraction $=4.11 \times 10^{-5}$ Exit mass flow rate $=0.0505 \mathrm{~kg} / \mathrm{sec}$ Contour line increment $=2 \%$ 


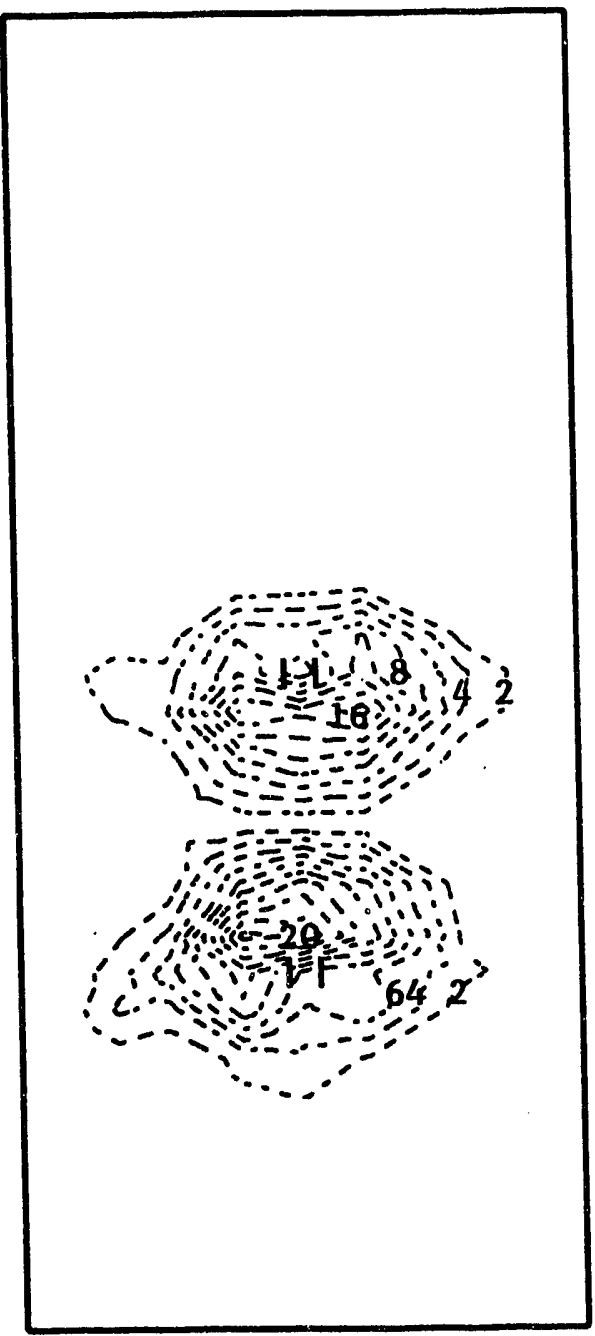

Fig. E.3. Constant air mass fraction lines (\%) at $I=5$ plane for Case 1.4

Inlet atr mass fraction $=8.21 \times 10^{-5}$ Exit mass flow rate $=0.0505 \mathrm{~kg} / \mathrm{sec}$ Contour line increment $=2 \%$ 


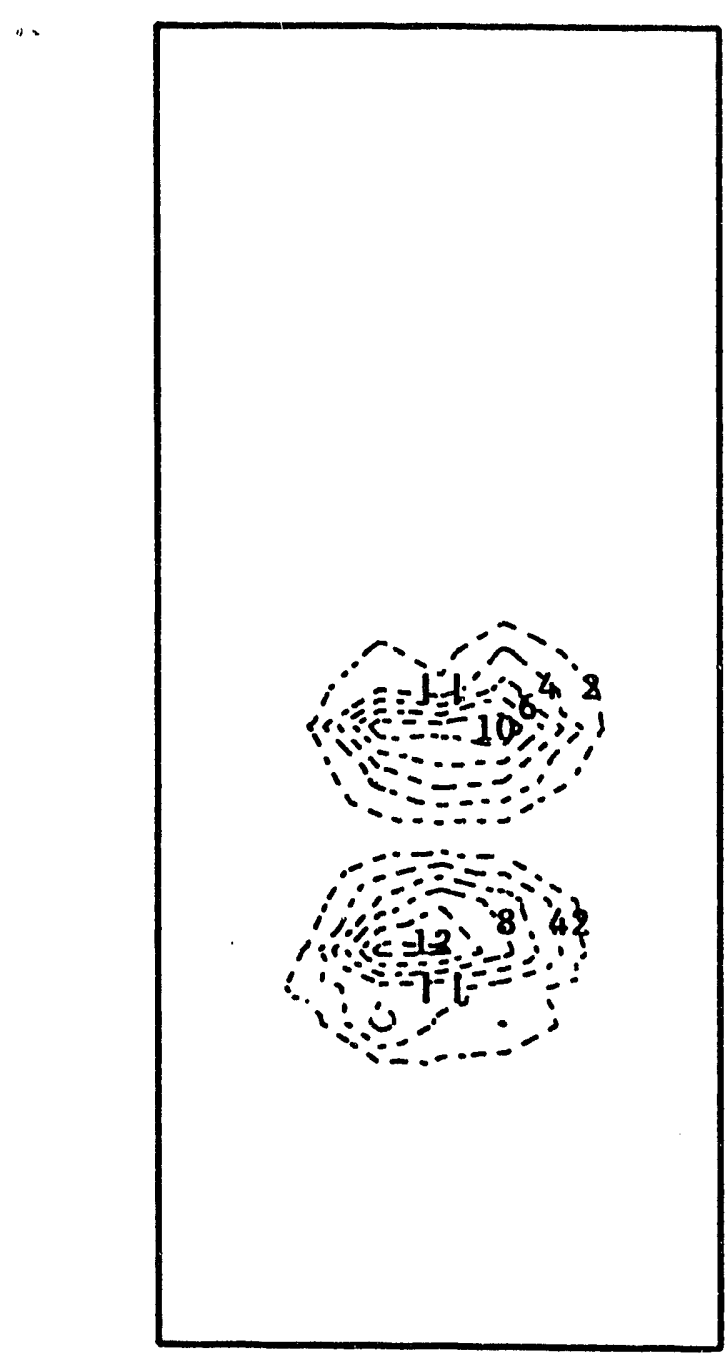

Fig. E.4. Constant air mass fraction lines (\%) at $I=5$ plane for Case 2.4

Inlet air mass fraction $=8.21 \times 10^{-5}$ Exit mass flow rate $=0.0101 \mathrm{~kg} / \mathrm{sec}$ Contour line increment $=2 \%$ 


\section{Distribution for ANL-92/2. Vol. II}

Internal:
T. H. Chien (5)
W. T. Sha (22)
R. W. Weeks
H. M. Domanus
C. E. Tull
C. A. Malefyt (2)
R. A. Valentin
ANL Patent
TIS Files

\section{External:}

DOE/OSTI (2)

B. Sheron, NRC, Washington, DC

ANL Libraries

ANL-E

ANL-W

Manager, Chicago Field Office, DOE

Materials and Components Technology Division Review Committee:

H. Birnbaum, University of Illinois at Urbana-Champaign. Urbana

R. Buchanan. University of Cincinnati, Cincinnati, OH

M. S. Dresselhaus, Massachusetts Institute of Technology, Cambridge MA

B. G. Jones, University of Illinois at Urbana-Champaign. Urbana

C.-Y. Li, Cornell University, Ithaca, NY

S. N. Liu, Electric Power Research Institute, Palo Alto, CA

R. E. Smith, Engineering Applied Sciences, Inc., Trafford, PA

E. Lin, Taiwan Power Company, for distribution (20) 

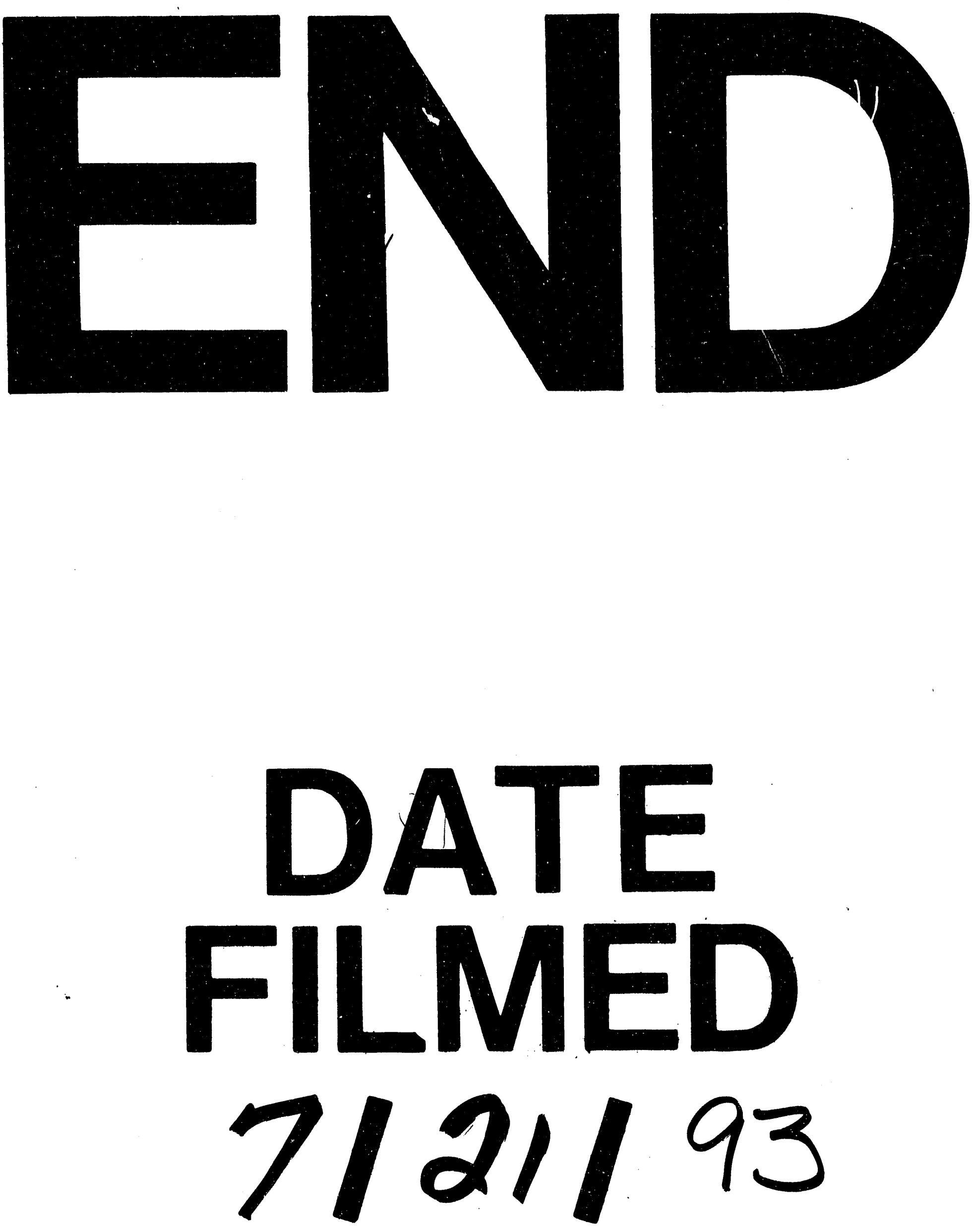
